Aus der Abteilung Psychiatrie und Psychotherapie

(Kommissarischer Leiter: Prof. Dr. med. B. Bandelow)

im Zentrum Psychosoziale Medizin

der Medizinischen Fakultät der Universität Göttingen

\title{
Die Auswirkungen von Nikotin und eines Nikotinentzugs auf polysomnografische und neuroendokrine Parameter
}

Eine systematische Übersichtsarbeit

\author{
INAUGURAL-DISSERTATION \\ zur Erlangung des Doktorgrades \\ der Medizinischen Fakultät \\ der Georg-August-Universität zu Göttingen
}

vorgelegt von

\section{Clara Landgraf}

aus Rastede 
Dekan:

1. Berichterstatterin: Prof. Dr. rer. nat. A. Rodenbeck

2. Berichterstatter: Priv.-Doz. Dr. med. T. Raupach, MME

3. Berichterstatter: PD S. Straube, BM BCh, MA, DPhil

Tag der mündlichen Prüfung: 14. Mai 2013 


\section{Inhaltsverzeichnis}

Abkürzungsverzeichnis $\quad$ IV

1 Einleitung 1

1.1 Einführung ............................ 1

1.2 Nikotin . . . . . . . . . . . . . . . . . . . . 2

1.2.1 Verbreitung des Tabakkonsums ............... 2

1.2.2 Gesundheitliche Folgen des chronischen Tabakkonsums . . . . . . . . . 3

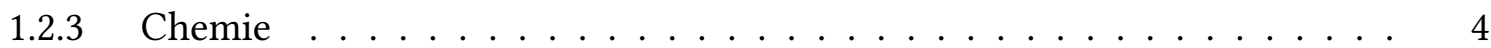

1.2.4 Pharmakokinetik ...................... 4

1.2.5 Der Nikotinrezeptor $\ldots \ldots \ldots \ldots \ldots$

1.2.6 Physiologische Wirkungen . . . . . . . . . . . . . 6

1.2.7 Tabak- und Nikotinabhängigkeit . . . . . . . . . . . . 8

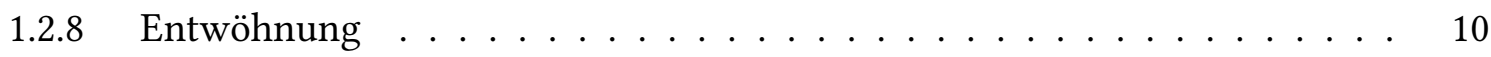

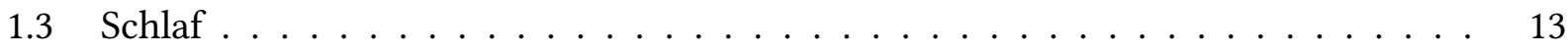

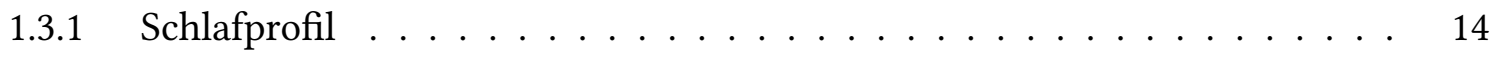

1.3.2 Physiologische Funktion . . . . . . . . . . . . . . . . 14

1.3.3 Regulation . . . . . . . . . . . . . . . . . 16

1.4 Dopamin . . . . . . . . . . . . . . . . . . . . . . . . . 19

1.4.1 Das Belohnungssystem . . . . . . . . . . . . . . . 20

1.5 Hypothalamus-Hypophysen-Nebennieren-Achse . . . . . . . . . . . . . 21

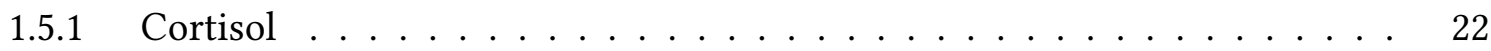

1.6 Der Zusammenhang zwischen Nikotin, Schlaf, Dopamin und HHN-Achse . . . . . 24 
1.6.1 Nikotin und Schlaf . . . . . . . . . . . . . . . . . . 24

1.6.2 Nikotin und Dopamin . . . . . . . . . . . . . . . . . . 25

1.6.3 Nikotin und HHN-Achse . . . . . . . . . . . . . . . . . . . 26

1.7 Fragestellung und Zielsetzung . . . . . . . . . . . . . . . . . . . . 27

2 Methoden $\quad 29$

2.1 Definitionen von Rauchgewohnheiten . . . . . . . . . . . . . . . . . . . 29

2.2 Polysomnografie . . . . . . . . . . . . . . . . . . . . . . . 29

2.3 Definitionen von Schlafvariablen . . . . . . . . . . . . . . . . . . 30

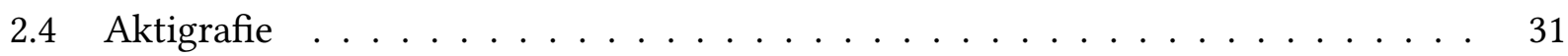

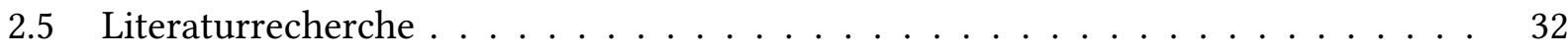

2.5.1 Objektive Schlafparameter . . . . . . . . . . . . . . . . . 34

2.5.2 Dopaminerges System . . . . . . . . . . . . . . . . . 36

2.5.3 Hypothalamus-Hypophysen-Nebennieren-Achse . . . . . . . . . 38

$\begin{array}{llr}3 & \text { Ergebnisse } & 41\end{array}$

3.1 Übersicht . . . . . . . . . . . . . . . . . . . . . . . . . 41

3.2 Objektive Schlafparameter . . . . . . . . . . . . . . . . . 43

3.2.1 Nikotingabe in tierexperimentellen Untersuchungen . . . . . . . . . . . 43

3.2.2 Nikotingabe an Nichtraucher . . . . . . . . . . . . . . . 48

3.2.3 Nikotinentzug bei Gelegenheitsrauchern . . . . . . . . . . . . . . . 54

3.2.4 Nikotinentzug bei Rauchern . . . . . . . . . . . . . . . 55

3.3 Dopaminerges System . . . . . . . . . . . . . . . . . . . . . . . . . 60

3.3.1 Nikotingabe an Nichtraucher . . . . . . . . . . . . . . . . . 60

3.3.2 Nikotinentzug bei Gelegenheitsrauchern . . . . . . . . . . . . . . 60

3.3.3 Nikotinentzug bei Rauchern . . . . . . . . . . . . . . . . 61

3.4 Hypothalamus-Hypophysen-Nebennieren-Achse . . . . . . . . . . . . . . . 63

3.4.1 Nikotingabe in tierexperimentellen Untersuchungen . . . . . . . . . 63

3.4 Nikotingabe an Nichtraucher . . . . . . . . . . . . . 72

3.4.3 Nikotinentzug bei Gelegenheitsrauchern . . . . . . . . . . . . . . . 73

3.4.4 Nikotinentzug bei Rauchern . . . . . . . . . . . . . . . . . . . . 75 
4 Diskussion $\quad 82$

4.1 Allgemeine Anmerkungen zu den Ergebnissen . . . . . . . . . . . . . . . . . 84

4.2 Anmerkungen zur Literaturrecherche . . . . . . . . . . . . . . . . . . 86

4.3 Ergebnisse der Literaturrecherche . . . . . . . . . . . . . . . . . . . 88

4.3.1 Objektive Schlafparameter . . . . . . . . . . . . . . . . 88

4.3.2 Dopaminerges System . . . . . . . . . . . . . . . . . . 99

4.3.3 Hypothalamus-Hypophysen-Nebennieren-Achse . . . . . . . . . . 104

4.4 Schlussfolgerungen und Ausblick . . . . . . . . . . . . . . . . . . . 116

$5 \quad$ Zusammenfassung $\quad 124$

6 Anhang: Tabellen 6.1-6.7 127

$\begin{array}{llr}7 & \text { Literaturverzeichnis } & 140\end{array}$

8 Abbildungsverzeichnis $\quad 160$

9 Tabellenverzeichnis $\quad 161$ 


\section{Abkürzungsverzeichnis}

$\begin{array}{ll}\text { ACh } & \text { Acetylcholin } \\ \text { ACT } & \text { Apomorphine-Challenge-Test } \\ \text { ACTH } & \text { Adrenocorticotropes Hormon } \\ \text { ADHS } & \text { Aufmerksamkeitsdefizit-Hyperaktivitätsstörung } \\ \text { ARAS } & \text { Aufsteigendes retikuläres Aktivierungssystem } \\ \beta 2-\text { KO } & \text { Beta2-Knockout } \\ \text { B2-nACh-R-Knockout } & \text { Beta2-nACh-Rezeptor-Knockout } \\ \text { BMI } & \text { Body-Mass-Index } \\ \text { BP } & \text { Bindungspotential } \\ \text { C } & \text { Cortisol } \\ \text { CBG } & \text { Cortisolbindendes Globulin } \\ \text { CRH } & \text { Corticotropin-Releasinghormon } \\ \text { CS } & \text { Corticosteron } \\ \text { CYP-450 } & \text { Cytochrom-P-450 } \\ \text { d } & \text { Tag(e) } \\ \text { D-Rezeptor } & \text { Dopaminrezeptor } \\ \text { DHEA(S) } & \text { Dehydroepiandrosteron(-sulfat) } \\ \text { DSM } & \text { Diagnostic and Statistical Manual of Mental Disorders } \\ \text { DST } & \text { Dexamethason-Suppressions-Test } \\ \text { EEG } & \text { Elektroenzephalografie } \\ \text { EMG } & \text { Elektromyografie } \\ \text { FTND } & \text { Fagerström Test for Nicotine Dependence } \\ \text { GABA } & \text { Gamma-Aminobuttersäure } \\ \text { GH } & \text { Growth-Hormone (Wachstumshormon) } \\ \text { h } & \text { Stunde(n) } \\ \text { HAM-D } & \text { Hamilton Rating Scale for Depression } \\ \text { HHN-Achse } & \text { Hypothalamus-Hypophysen-Nebennieren-Achse } \\ \text { ICD } & \text { International Classification of Diseases } \\ \text { i.c.v. } & \text { Intrazerebral } \\ \text { i.p. } & \text { Intraperitoneal } \\ \text { i.v. } & \text { Intravenös } \\ & \end{array}$




\begin{tabular}{|c|c|}
\hline kg & Kilogramm \\
\hline LDL & Low-Density-Lipoprotein \\
\hline LE & Long-Evans \\
\hline m & Männlich \\
\hline mACh-Rezeptor & Muskarinischer Acetylcholin-Rezeptor \\
\hline mg & Milligramm \\
\hline$\mu \mathrm{g}$ & Mikrogramm \\
\hline $\min$ & Minuten \\
\hline MSLT & Multipler Schlaf-Latenztest \\
\hline MT & Movement Time \\
\hline nACh-Rezeptor & Nikotinischer Acetylcholin-Rezeptor \\
\hline NN & Nebenniere \\
\hline NNM & Nebennierenmark \\
\hline NP & Nikotinpflaster \\
\hline NR & Nichtraucher \\
\hline NREM & Non-Rapid-Eye-Movement \\
\hline PET & Positronen-Emissions-Tomografie \\
\hline PGO-Wellen & Ponto-geniculo-okzipitale Wellen \\
\hline PLM & Periodic-Leg-Movements \\
\hline PSG & Polysomnografie \\
\hline PVN & Paraventrikulärer Nukleus \\
\hline $\mathbf{R}$ & Raucher \\
\hline REM & Rapid-Eye-Movement \\
\hline RLS & Restless-Legs-Syndrom \\
\hline s.c. & Subkutan \\
\hline SCN & Nucleus suprachiasmaticus \\
\hline SD & Sprague-Dawley \\
\hline SWS & Slow-Wave-Sleep \\
\hline TST & Total-Sleep-Time (Gesamtschlafzeit) \\
\hline VLPO & Nucleus präopticus venterolateralis \\
\hline $\mathbf{W}$ & Wach \\
\hline $\mathbf{w}$ & Weiblich \\
\hline WHO & World Health Organization \\
\hline WT & Wildtyp \\
\hline WZ & Wachzeit \\
\hline ZNS & Zentrales Nervensystem \\
\hline$\uparrow$ & Signifikant erhöht \\
\hline$\leftrightarrow$ & Gleichbleibend \\
\hline$\downarrow$ & Signifikant erniedrigt \\
\hline
\end{tabular}




\section{Einleitung}

\subsection{Einführung}

Gegenwärtig konsumiert etwa ein Viertel der Menschheit Tabakprodukte (World Health Organization, 2011), obwohl die negativen gesundheitlichen Folgen allgemein bekannt sind. Verantwortlich hierfür ist zu einem großen Teil die Entstehung einer Nikotinabhängigkeit durch den Konsum von Tabakprodukten, die einer von vielen Rauchern erwünschten Abstinenz entgegenwirkt.

Reizbarkeit, Nervosität, Verschlechterung der Stimmung bis hin zu Depression und Schlaflosigkeit sind die im Entzug häufig berichteten Symptome, die oft zum Rückfall in die Nikotinsucht führen. Die neurobiologischen Korrelate dieser Entzugssymptome sowie die Wirkungen des Nikotins auf die physiologischen Systeme sind Gegenstand aktueller Forschung. Da bekannt ist, dass Nikotin die Ausschüttung diverser Transmitter beeinflusst, die an der Regulation des Schlafs beteiligt sind, verwundert es nicht, dass sowohl Tabakkonsum als auch der Entzug von Tabak Schlafstörungen hervorrufen können.

Unter den durch Nikotin beeinflussten Transmittern sind auch das Glucocorticoid Cortisol und das Catecholamin Dopamin. Cortisol als Hormon der Hypothalamus-Hypophysen-Nebennieren-Achse (HHN-Achse) und Dopamin als integraler Bestandteil des bei einer Abhängigkeit aktivierten Belohnungssystems sind eng mit dem Schlaf-Wach-Zyklus verknüpft. Um die Auswirkungen des Nikotinkonsums und eines Nikotinentzugs zu erfassen, ist es somit sinnvoll, den Schlaf, die Transmitter der HHN-Achse und Dopamin gemeinsam zu betrachten.

Um zu verstehen, welche Veränderungen durch Nikotinkonsum und Nikotinentzug bezüglich des Schlafs, der HHN-Achse und des dopaminergen Systems entstehen, wurden bereits einige Studien durchgeführt. Die vorliegende Arbeit fasst nach aktuellem Kenntnisstand als erste die 
zu diesem Thema veröffentlichten Studien durch eine systematische Literaturrecherche zu einer Übersichtsarbeit zusammen. Die Zusammenfassung der Studienergebnisse kann dabei helfen, die Nikotinabhängigkeit sowie den Nikotinentzug besser zu verstehen und gegebenenfalls Prädiktoren für einen Rückfall zu ermitteln. Mit diesem Wissen und geeigneten Maßnahmen könnte den Rauchern in Zukunft die Abstinenz erleichtert und die durch das Rauchen verursachte Morbidität und Mortalität reduziert werden.

\subsection{Nikotin}

Das Nikotin erhielt seinen Namen zu Ehren des französischen Diplomaten Jean Nicot, der im 16. Jahrhundert Tabak als Heilpflanze aus Amerika nach Europa brachte. Das Nikotin entsteht in der Wurzel der Tabakpflanze, von wo es in die Blätter transportiert und gespeichert wird. Dort dient es der Pflanze zur Insektenabwehr (Soloway, 1976).

Aus den Blättern der Tabakpflanze werden durch Trocknung, Fermentation und Lagerung unterschiedlichste Tabakwaren hergestellt, die weltweit konsumiert werden.

\subsubsection{Verbreitung des Tabakkonsums}

Aus dem Mikrozensus des Statistischen Bundesamtes geht hervor, dass im Jahr 2009 in Deutschland insgesamt 27,6 \% der Befragten, die älter als 15 Jahre waren, rauchten. Seit 1999 ist der Anteil der rauchenden Bevölkerung gesunken, so rauchten zehn Jahre zuvor noch 29,2 \% der Befragten (Statistisches Bundesamt, 2011). Global betrachtet rauchten 2006 41,1\% der Männer und 8,9\% der Frauen. Das bedeutet, dass etwa ein Viertel der Menschheit zu diesem Zeitpunkt regelmäßig rauchte (World Health Organization, 2011). Zwar ist das Einstiegsalter in den Tabakkonsum heute niedriger als vor 50 Jahren (Statistisches Bundesamt, 2011), aber auch in der Altersgruppe der 15bis 20-Jährigen wird seit 1999 ein Rückgang der Raucherquote verzeichnet (Statistisches Bundesamt, 2010).

Betrachtet man die historische Entwicklung des Rauchverhaltens der deutschen Bevölkerung, so kann beobachtet werden, dass vor dem ersten Weltkrieg das Rauchen meist den oberen Gesellschaftsschichten vorbehalten war. Mit Erfindung der maschinellen Produktion von Zigaretten 
gegen Ende des 19. Jahrhunderts fand ein Wandel statt, es rauchten nun zunehmend Menschen niedriger Gesellschaftsschichten, hier vor allem die Männer. Mit Beginn der Frauenbewegung stieg ab 1945 der Anteil der Raucherinnen bis Mitte der 1960er Jahre an (Pötschke-Langer et al., 2009).

\subsubsection{Gesundheitliche Folgen des chronischen Tabakkonsums}

Beim Rauchen des Tabaks entsteht ein Aerosol, welches von den Konsumenten inhaliert wird und über 7000 identifizierte oder teilweise identifizierte Verbindungen enthält, die z. T. nachgewiesenermaßen krebserregend sind. Im Jahr 2007 verstarben in Deutschland an den Folgen des Tabakkonsums schätzungsweise 110000 Menschen, das sind $13 \%$ aller Todesfälle. Etwa ein Drittel dieser Menschen starb an Lungenkrebs und ein Sechstel an ischämischen Herzerkrankungen (Pötschke-Langer et al., 2009). Weltweit sind im Jahr 2000 4,2 Millionen Menschen an den Folgen chronischen Tabakkonsums gestorben (Mackay und Eriksen, 2002). Mehr als jeder zweite Raucher stirbt vorzeitig, so erreichen $81 \%$ der Nichtraucher ein Alter von 70 Jahren, bei den Rauchern sind dies hingegen nur 58 \% (Pötschke-Langer et al., 2009). Im Durchschnitt sterben Raucher zehn Jahre früher als Nichtraucher (Doll et al., 2004).

Die häufigsten gesundheitlichen Schäden und Risiken, die durch das Rauchen verursacht werden, sind im Folgenden aufgeführt. Für einen Großteil der Erkrankungen ist nicht Nikotin, sondern sind andere Inhaltsstoffe des Tabaks oder des Tabaksrauchs verantwortlich.

- Kardiovaskuläre Erkrankungen wie Arteriosklerose, Thrombose, koronare Herzkrankheit, Herzinfarkt, periphere arterielle Verschlusskrankheit und Schlaganfall (U. S. Department of Health und Human Services, 2004; Chi und Jaff, 2008).

- Respiratorische Erkrankungen wie Pneumonie, Bronchitis und chronisch obstruktive Lungenerkrankung (U. S. Department of Health und Human Services, 2004; Dekant und Vamvakas, 2005).

- Maligne Neoplasien in der Mundhöhle, im Kehlkopf, in der Lunge, im Magen-Darm-Trakt, in der Niere, in der Blase, im Gebärmutterhals und im Blut (akute myeloische Leukämie) (U. S. Department of Health und Human Services, 2004; Böcker et al., 2004). 
- Komplikationen in der Schwangerschaft (Böcker et al., 2004).

\subsubsection{Chemie}

Der chemische Stoff Nikotin ist ein farbloses und visköses Öl, welches sich an der Luft braun verfärbt. Mit einem pKa-Wert von 8 ist es eine schwache Base. Nikotin ist ein tertiäres Amin mit zwei Stereoisomeren, $(R)$ - und (S)-Nikotin, wobei letzteres das aktive Isomer ist, welches an den nikotinischen Acetylcholin-Rezeptor (nACh-Rezeptor) bindet. Nikotin gehört zu den SolanaceenAlkaloiden und ist das Hauptalkaloid des Tabaks. Zudem wird es als schnellwirkendes und starkes Gift bezeichnet.

\subsubsection{Pharmakokinetik}

Resorption Das im Zigarettenrauch enthaltene Nikotin wird über die Schleimhäute des Mundes und des Respirationstrakts resorbiert. Die Menge des aufgenommenen Nikotins hängt zum einen vom Nikotingehalt und $\mathrm{pH}-$ Wert des Tabaks und von der Art des Rauchvorgangs (z. B. Tiefe der Inhalation) ab, zum anderen von der chemischen Beschaffenheit des Nikotins: Ist das Nikotinmolekül sauer, wird es schlecht in die Körpergewebe aufgenommen. Da für die Zigarettenproduktion saure Tabaksorten verwendet werden, muss der Raucher inhalieren, um so die Kontaktfläche der Schleimhäute und somit die Menge des resorbierten Nikotins zu vergrößern. Des Weiteren wird der pH-Wert des Nikotins im neutralen Lungengewebe abgepuffert und so kann das Nikotin leichter in das Gewebe eindringen. Nikotin wird als Base sehr viel besser aufgenommen und deshalb nutzen Zigarettenhersteller Zusatzstoffe wie Harnstoff, um den Tabak zu alkalisieren. Der Tabak in Pfeifen oder Zigarren ist bereits alkalisch und so kann das Nikotin leicht über die Schleimhaut des Mundes aufgenommen werden. Pro gerauchte Zigarette wird unterschiedlich viel Nikotin resorbiert, abhängig von der Art des Rauchens und dem Nikotingehalt der Zigarette. Benowitz und Jacob (1984) ermittelten, dass pro Zigarette durchschnittlich 1 mg Nikotin resorbiert wird.

Verteilung Das resorbierte Nikotin gelangt in den Blutkreislauf und wird schnell über die Lungenvenen und die linke Herzkammer im gesamten Organismus verteilt. Inhalierte Stoffe umgehen den intestinalen und hepatischen First-Pass-Effekt und so erreicht Nikotin innerhalb 
von 10-20 Sekunden das Gehirn (Zevin et al., 1998). In diesem Zusammenhang ist Folgendes von Bedeutung: Je schneller eine Substanz resorbiert und in das Gehirn transportiert wird, je schneller also das Anfluten des Stoffs, desto höher ist die positive Verstärkung (Benowitz, 2008a). Andere Applikationsformen von Nikotin, die meist als Ersatztherapien im Nikotinentzug eingesetzt werden, erreichen diese extrem schnelle Anflutung im Gehirn nicht. Das Nikotin verteilt sich rasch im gesamten Blutkreislauf und wirkt auf die unterschiedlichsten Gewebe.

Konzentrationsverlauf Nachdem die Nikotinkonzentration im Blut während des Konsums einer Zigarette steil angestiegen ist, fällt sie innerhalb von 20-30 Minuten langsam ab, da das Nikotin sich in den Körpergeweben verteilt. Insbesondere der Skelettmuskel nimmt Nikotin auf (Le Houezec, 2003). Im Laufe eines Tages steigt der Nikotinspiegel im Blut der Raucher kontinuierlich an. Jede gerauchte Zigarette verursacht eine Spitze und die Nikotinkonzentration steigt, da das Nikotin im Körper akkumuliert. In der Nacht fällt die Konzentration des Nikotins durch die Elimination ab.

Abbau Nikotin besitzt eine Halbwertszeit von circa zwei Stunden und wird auf unterschiedlichen Wegen aus dem Körper eliminiert. $10 \%$ des resorbierten Nikotins werden unverändert mit dem Harn ausgeschieden, der Großteil hingegen wird oxidativ in der Leber abgebaut. Metaboliten hierbei sind u. a. Cotinin, Nikotin-N-Oxid und Nikotinglucuronid, wobei Cotinin mit etwa $70 \%$ den größten Anteil bildet. Cotinin ist pharmakologisch unwirksam, besitzt eine Halbwertszeit von circa 16 Stunden und kann somit eine Messgröße für die aufgenommene Nikotinmenge darstellen. In vielen Studien, in denen ein Nikotinentzug untersucht wird, wird Cotinin bestimmt, um die Abstinenz der Probanden zu kontrollieren.

Das in der Leber lokalisierte Enzymsystem, darunter als wichtigste Vertreter die Cytochrom-P-450Enzyme (CYP-450), verstoffwechselt Nikotin. Für den Abbau ist insbesondere der Subtyp CYP2A6 und in geringerem Umfang auch CYP2B6 verantwortlich.

Neben genetisch determinierten Verstoffwechslungsraten spielen auch andere Faktoren eine Rolle, die den Abbau von Nikotin beeinflussen können. Dies sind u. a. physiologische Gegebenheiten 
wie Alter, Rasse und Geschlecht, Nahrungsmittel, Mahlzeiten, die Durchblutungsrate der Leber, Krankheiten, Medikamente und das Rauchen selbst (Hukkanen et al., 2005).

\subsubsection{Der Nikotin rezeptor}

Rezeptoren, die den Neurotransmitter Acetylcholin ( $\mathrm{ACh}$ ) binden, heißen Acetylcholin-Rezeptoren oder Cholinozeptoren. Es sind Transmembranrezeptoren, die in der Zellmembran verschiedenster Körperzellen vorkommen, beispielsweise prä- und postsynaptisch auf Nervenzellen. Sie erfüllen je nach Lokalisation und molekularer Struktur unterschiedlichste Aufgaben. Es sind zwei unterschiedliche Subtypen bekannt, zum einen der nikotinische (nACh-Rezeptor), zum anderen der muskarinische ACh-Rezeptor (mACh-Rezeptor) - benannt nach dem Bindungsverhalten der natürlich vorkommenden Liganden Nikotin und Muskarin. Der nACh-Rezeptor bindet mit hoher Affinität Nikotin und wird Nikotinrezeptor genannt. Strukturell besteht dieser Rezeptor aus $\alpha$ und $\beta$-Untereinheiten und je nach Kombination derselben entstehen unterschiedliche Subtypen. Ein Subtyp, der im zentralen Nervensystem (ZNS) der Menschen am häufigsten vorkommt, bindet Nikotin mit hoher Affinität. Es ist ein Rezeptor mit den Untereinheiten $\alpha 4$ und $\beta 2$ (Benowitz, 2008b).

\subsubsection{Physiologische Wirkungen}

Bei der Bindung von Nikotin an die Nikotinrezeptoren kommt es zur Ausschüttung von Neurotransmittern (Benowitz, 2008a). Hierbei kann Nikotin ein bivalentes Wirkungsspektrum zugeschrieben werden (Heinz und Batra, 2003). In Abhängigkeit von Nikotindosis und der individuellen Ausgangssituation des Organismus kommt es zu unterschiedlichen Effekten. In niedrigen Dosen stimuliert Nikotin das neuronale System, verbunden mit einer Steigerung der Herz- und Atemfrequenz und der Stoffwechselrate, einem Anstieg des Blutdrucks und einer Zunahme der Aufmerksamkeit, der Konzentration und der psychomotorischen Leistungsfähigkeit (Levin et al., 1998; Benowitz et al., 1982; Kröger und Lohmann, 2007). Höhere Dosen hingegen bewirken beispielsweise eine Abnahme von Aggressivität, Nervosität, Angst oder Depressivität, verbunden mit Beruhigung und Entspannung (Kröger und Lohmann, 2007). Erfahrene Raucher können durch Frequenz und Tiefe der Inhalation die gewünschte Wirkung herbeiführen (Heinz und Batra, 2003). 
Eine Überdosierung von Nikotin kann zu Erbrechen, Kopfschmerzen, Tachy- oder Bradykardien, Hypotonie und -thermie, Antidiurese, Diarrhoe und Tremor führen (Zevin et al., 1998; Heinz und Batra, 2003). Ein Teil dieser Symptome lässt sich aus der Blockade der ganglionären Erregungsleitung erklären, da es zu einer anhaltenden Depolarisation der Zellmembranen kommt. Die orale letale Dosis für den Menschen liegt bei 50 mg Nikotin (Pangritz, 2003); andere Quellen sprechen von $1 \mathrm{mg}$ Nikotin/kg Körpergewicht (Heinz und Batra, 2003). Nach der Aufnahme einer solchen Dosis kommt es zu einer zentralen Erregung mit Krämpfen, Koma, Kreislaufkollaps und Atemlähmung. Bei ausreichend hohen Dosen kann der Tod innerhalb weniger Minuten durch Hemmung der neuromuskulären Übertragung eintreten (Haustein, 2001).

Betrachtet man die Wirkungen des Nikotins im Tagesprofil, zeigt sich ein täglich wiederkehrender Zyklus. Die erste Zigarette des Tages erzeugt erhebliche pharmakologische Effekte, vornehmlich anregender Natur. Ursache hierfür sind die vielen unbesetzten Nikotinrezeptoren und die damit verbundene stärkste Freisetzung von Neurotransmittern des Tages, wie z. B. von Dopamin. Dennoch entwickelt sich hierbei schon eine gewisse Toleranz bezüglich der gewünschten Wirkungen, da die Nikotinrezeptoren zunehmend unempfindlich werden (Desensibilisierung). Raucher erlernen, wann diese Toleranz zurückgeht, und konsumieren dann eine zweite Zigarette. Im Laufe des Tages akkumuliert das Nikotin im Körper, was zu einer weiteren Abnahme der Wirkungen führt. Dadurch wird die Motivation für den Zigarettenkonsum mehr und mehr von Entzugserscheinungen bestimmt. Die Abstinenz über Nacht sorgt für eine Resensibilisierung der Nikotinwirkungen und die erste Zigarette am Morgen erzeugt wieder die gewünschten anregenden Effekte (Dani und Heinemann, 1996; Benowitz, 2008a).

Die akuten, reversiblen Toleranzentwicklungen müssen von langsam stattfindenden chronischen Anpassungsvorgängen im ZNS von Rauchern abgegrenzt werden. Bei chronischem Nikotinkonsum findet dort eine Neuroadaptation statt. Dies bedeutet, dass die Struktur des ZNS durch anhaltenden Nikotinkonsum verändert wird - so steigt z. B. die Anzahl von Nikotinrezeptoren (Up-Regulation). Dieser Umstand scheint als Kompensation stattzufinden, da die bereits vorhandenen Rezeptoren durch die Nikotinbindung desensibilisiert sind (Benowitz, 2008a,b). Fehlt Nikotin, z. B. während des Schlafens oder eines Entzugs, sind mehr Rezeptoren unbesetzt, was zu einer erhöhten und abnormen Erregung des cholinergen Systems führt. Symptome wie körperliche Unruhe und Unbehagen 
treten auf. Der Raucher therapiert sich selbst, indem er die Nikotinrezeptoren durch den Konsum von Nikotin desensibilisiert und so eine normale Anzahl an erregbaren Rezeptoren herbeiführt (De Biasi und Dani, 2011; Dani und Heinemann, 1996). Neben den zentralen Toleranzentwicklungen finden diese auch peripher statt. Nikotindosen, die toxisch für nikotinnaive Personen wären, werden von abhängigen Rauchern kardiovaskulär und gastrointestinal gut toleriert (Heinz und Batra, 2003).

\subsubsection{Tabak- und Nikotinabhängigkeit}

\section{Definition der Tabak- und Nikotinabhängigkeit}

Weltweit definieren die zwei wichtigsten diagnostischen Klassifikationssysteme die Tabak- bzw. Nikotinabhängigkeit: Die International Classification of Diseases (ICD) der World Health Organization (WHO) und das Diagnostic and Statistical Manual of Mental Disorders (DSM) der American Psychiatric Association. Zusammenfassend ist die Abhängigkeitserkrankung durch die Unfähigkeit zur Abstinenz, einen Kontrollverlust, eine Toleranzentwicklung und das Auftreten von Entzugserscheinungen in Abstinenz gekennzeichnet (Heinz und Batra, 2003).

\section{Der Fagerström-Test}

Um den Schweregrad der Tabakabhängigkeit zu bestimmen, gibt es eine Vielzahl verschiedener Tests. Einer der bekanntesten ist von Karl-Olov Fagerström (Heatherton et al., 1991): The Fagerström Test for Nicotine Dependence (FTND). Die beiden wichtigsten Fragen zur Einschätzung der tatsächlichen Abhängigkeitsstärke sind hierbei die Fragen nach dem Zeitpunkt der ersten Zigarette am Morgen und die Anzahl der gerauchten Zigaretten pro Tag (Kröger und Lohmann, 2007). Das Ergebnis dieses Tests korreliert gut mit den Kohlenmonoxidwerten im Blut der Raucher, den Tabakfolgekrankheiten und dem Schweregrad des Entzugs (Lesch und Walter, 2009). Zudem dient das Testergebnis als Prädiktor für den Entzugserfolg. Ein hoher Wert spricht für eine geringere Chance, erfolgreich abstinent zu bleiben, und umgekehrt korreliert ein niedriger Wert mit einer höheren Chance dauerhaft in Abstinenz zu leben. Diese Einordnung kann bei der Wahl der unterstützenden Therapie im Nikotinentzug hilfreich sein (Kröger und Lohmann, 2007). 


\section{Erklärungsmodelle der Abhängigkeit}

Viele verschiedene Modelle erklären die Entstehung und Aufrechterhaltung einer Abhängigkeit. Diese Modelle entstammen z. B. der Psychologie, Neurobiologie oder Soziologie. Ein Zusammenspiel von persönlichen Faktoren (darunter genetische Aspekte), Charakteristika der Droge, soziales Umfeld und andere Faktoren machen die Erklärungsansätze so vielfältig. Ein eindeutiges Erklärungsmodell existiert nicht und Vieles ist noch unerforscht. Auch sollte man einzelne Theorien nicht isoliert betrachten, da viele Erklärungsansätze ineinandergreifen.

Psychologischer Ansatz Es existiert eine Vielzahl psychologischer Erklärungsmodelle, darunter tiefen-, trieb-, ich- und objektpsychologische Ansätze. An dieser Stelle wird das lerntheoretische Modell zur Erklärung einer Abhängigkeit dargestellt, da dieses Modell auch einen neuropsychologischen Ansatz bietet. Die neurobiologische Erklärung für die Entstehung einer Abhängigkeit wird in 1.4.1 auf Seite 20 behandelt.

Wendet man die Theorie des instrumentellen Lernens zur Erklärung des Konsums einer Substanz an, kann die Entstehung einer Abhängigkeit folgendermaßen veranschaulicht werden: Das Verhalten, hier der Konsum von Tabakprodukten, wird mit positiven und negativen Verstärkern verknüpft. Positive Verstärker sind z. B. die angenehmen Wirkungen des Nikotins wie die Aktivierung des Belohnungssystems. Diese Mechanismen sind insbesondere bei der Entstehung einer Abhängigkeit von Bedeutung. Fallen unangenehme Zustände (wie Entzugserscheinungen) durch den Konsum weg, spricht man von negativer Verstärkung und diese trägt maßgeblich zur Aufrechterhaltung des süchtigen Verhaltens bei. Die Reaktionen auf den Nikotinkonsum werden erlernt und gespeichert. Auch können zuvor neutrale Reize, wie z.B. das Anfassen eines Feuerzeugs oder der Aufenthalt an Orten, an denen man „gelernt“ hat zu rauchen, als sogenannte sekundäre Verstärker auftreten. Diese Reize sind nun mit der Aufnahme des Tabakprodukts verknüpft und können das Verlangen danach auslösen. Gerade die sekundären Verstärker, wie z. B. auch die sensorischen Aspekte des Rauchens, verhindern oft den Erfolg eines Entzugs (Rist und Watzl, 1999). 


\subsubsection{Entwöhnung}

Aufgrund der enormen Gesundheitsrisiken, die der Tabakkonsum mit sich bringt, wollen sich viele Raucher das Rauchen abgewöhnen. Der Nutzen der Raucherentwöhnung kann in einer britischen Studie an über 34000 Menschen, die über 50 Jahre beobachtet wurden, belegt werden. Aus dieser geht hervor, dass ein Rauchstopp im Alter von 60, 50, 40 oder 30 Jahren einen Gewinn von drei, sechs, neun oder zehn Lebensjahren mit sich bringt (Doll et al., 2004).

\section{Entzug}

Die Erfolgsquoten von Abstinenzversuchen betragen ohne therapeutische Maßnahmen lediglich 0,5-3\% (Cornuz et al., 2004). Ein Grund für dieses schlechte Ergebnis ist die durch die bestehende Sucht ausgelöste Entzugssymptomatik. Wie bereits unter 1.2 .6 auf Seite 6 beschrieben, ist die Neuroadaptation im Raucherhirn die Grundlage für Entzugserscheinungen. Schwindel, Kopfschmerzen, Schlafstörungen, Reizbarkeit und Depressionen sind einige der Symptome. Im Nikotinentzug kann ein charakteristisches Entzugssyndrom auftreten, welches nach diagnostischen Kriterien aus dem DSM-4 definiert ist. Die Entzugssymptomatik tritt innerhalb weniger Stunden auf und erreicht ihr Maximum nach 24 bis 48 Stunden. Über die folgenden Wochen nach Rauchstopp nehmen die Beschwerden dann meist ab (Cornuz et al., 2004). In einer Veröffentlichung von Hughes (1992) verschwanden Entzugserscheinungen wie Unruhe, Konzentrationsschwierigkeiten, Reizbarkeit oder Rastlosigkeit nach 30 Tagen. Lediglich der gesteigerte Appetit und die damit einhergehende Gewichtszunahme gingen zu diesem Zeitpunkt noch nicht zurück. Um die Entzugserscheinungen zu lindern und den Nikotinentzug erfolgreich werden zu lassen, existiert eine Vielzahl von therapeutischen Hilfen.

\section{Therapeutische Interventionen in der Raucherentwöhnung}

Um Rauchern den Ausstieg aus dem Nikotinkonsum zu erleichtern, wurden viele verschiedene Methoden, Medikamente und Interventionen auf ihren Nutzen hin untersucht. Eingeteilt werden können diese Therapien in unterschiedliche Gruppen, wie z. B. in medikamentöse oder psychologi- 
sche Maßnahmen. Im Folgenden werden einige häufig angewandte therapeutische Interventionen in der Raucherentwöhnung dargestellt.

Psychologische Verfahren Zu den psychologischen Entwöhnungsmethoden zählen Selbsthilfegruppen, Verhaltenstherapien und Entspannungsverfahren. Viele Maßnahmen können in der Gruppe oder als Einzelperson wahrgenommen werden. In speziellen Nichtraucherkursen werden Kombinationen verschiedener psychologischer Methoden angeboten. So stützt sich die Verhaltenstherapie auf die Annahme des lerntheoretischen Modells der Abhängigkeit, in der das erlernte Rauchverhalten wieder verlernt werden muss (Batra, 2000). Beratung, Abstinenzvorbereitung, Konsumbeendigung und Erfolgsstabilisierung sind die Eckpfeiler im verhaltenstherapeutischen Konzept. Das persönliche Rauchverhalten und die Beweggründe für den Rauchstopp werden analysiert und Strategien zur Rückfallprophylaxe erlernt.

Medikamentöse Verfahren Als pharmakologische Unterstützung im Nikotinentzug sind in Deutschland u. a. Nikotinersatztherapien, das Antidepressivum Bupropion und der Arzneistoff Vareniclin zugelassen. In der Nikotinersatztherapie werden unterschiedliche Nikotinpräparate angeboten, darunter Pflaster, Kaugummis, Lutschtabletten, Inhalatoren und Nasensprays. Je nach Stärke der Nikotinabhängigkeit, den Rauchgewohnheiten und Vorlieben kann zwischen den Präparaten und ihren Dosierungen gewählt werden. Auch Kombinationen verschiedener Applikationsformen sind möglich. Allen Präparaten gemein ist die Substitution von Nikotin, um durch Erreichen eines gewissen Nikotinspiegels Entzugserscheinungen zu mindern und Belohnungseffekte im dopaminergen Verstärkungssystem zu erzeugen (Talwar et al., 2004). Die Präparate unterscheiden sich allerdings stark in ihren pharmakologischen Eigenschaften. Während die Pflaster kontinuierlich Nikotin abgeben und keine schnelle Anflutung im ZNS bewirken, wird die positive Verstärkung (der Belohnungseffekt) besser durch die schnellere Resorption bei Verwendung von Nasensprays und Inhalatoren erreicht. Neben diesen Eigenschaften könnte ein weiterer Wirkmechanismus in der Desensibilisierung der Nikotinrezeptoren liegen. Sind diese durch die Nikotinersatztherapie bereits unerregbar, löst das Nikotin aus Tabakprodukten nur noch geringe belohnende Wirkungen aus und es kommt womöglich zu einer Nikotinintoxikation, wenn während der Nikotinersatztherapie geraucht wird (Benowitz, 2008a; Talwar et al., 2004). 
Das Antidepressivum Bupropion erhöht als selektiver Dopamin- und Noradrenalin-Wiederaufnahmehemmer die Dopamin- und Noradrenalinlevel im Gehirn. Hierdurch simuliert Bupropion die Wirkung von Nikotin, zudem blockiert es die Nikotinrezeptoren, was zu einer geringeren Wirkung von Tabakprodukten führt.

Vareniclin ist ein partieller Agonist an $\alpha 4-\beta 2$-Nikotinrezeptoren und erzeugt ähnliche belohnende Effekte wie Nikotin selbst. Rauchverlangen und Entzugssymptome werden verringert. Auch an dieser Stelle spielen Desensibilisierungsprozesse eine Rolle: Blockiert Vareniclin einige Nikotinrezeptoren, kann das Nikotin aus dem Tabak nicht seine volle Wirkung entfalten und der Verstärkereffekt nimmt ab.

Neben diesen Medikamenten gibt es weitere Präparate, die in der Raucherentwöhnung eingesetzt werden, darunter Clonidin und Nortriptylin, auf die hier nicht weiter eingegangen wird.

Andere Methoden Unter den zahllosen Methoden zur Raucherentwöhnung finden sich auch Akupunktur und Hypnose. Bisher konnte allerdings nicht eindeutig bewiesen werden, dass Akupunktur, Akupressur, Lasertherapie, Elektrostimulation oder Hypnose im Nikotinentzug von Nutzen sind. Geschuldet werden kann dies u. a. den methodischen Problemen bei Durchführung klinischer Studien mit den erwähnten Therapien.

Die Erfolgsquoten der einzelnen Entwöhnungstherapien variieren in der Literatur. Nach Schunack (2008) beträgt die Ein-Jahres-Abstinenzrate für die Verhaltenstherapie $13 \%$, für die Nikotinersatztherapie 14\%, für Bupropion $16 \%$ und für Vareniclin $22 \%$. Eine Kombination verschiedener Methoden kann die Wahrscheinlichkeit eines erfolgreichen Entzugs signifikant erhöhen, wenn z. B. eine Verhaltenstherapie und ein Nikotinersatz angeboten werden (Niaura, 2008).

\section{Rückfall}

Die Ursachen für einen Rückfall in die Nikotinsucht sind komplex und vielfältig, da hierbei biologische, soziale und psychische Aspekte eine Rolle spielen. Die meisten abstinenten Raucher werden innerhalb von drei Monaten rückfällig. Jeder Zweite dieser Rückfälligen greift bereits nach zwei Tagen wieder zum Tabak (Jain, 2003). Biologisch betrachtet ist der Rückfall eine Beendigung 
von Entzugssymptomen, die durch die strukturellen Änderungen im ZNS von abhängigen Rauchern hervorgerufen werden. Durch erneuten Nikotinkonsum werden die übermäßig exprimierten Nikotinrezeptoren besetzt und ein Normalmaß an cholinerger Erregung wiederhergestellt. Die unangenehmen Symptome lassen nach und es wird vermehrt Dopamin im Belohnungssystem freigesetzt, was zu Genuss und Glücksgefühlen führt. Hierbei scheint auch das möglicherweise adaptierte dopaminerge System des Abhängigen eine Rolle zu spielen, da dies eventuell verstärkt auf einen erneuten Nikotinkonsum reagiert (Heinz und Batra, 2003). Einen biologischen Ansatz in Rückfallmodellen bietet auch die Genetik der Raucher, welche es z. B. durch strukturelle Unterschiede im ZNS entweder leichter oder schwerer haben, mit dem Rauchen aufzuhören. Ein hierbei diskutierter Ansatz ist u. a. der Polymorphismus im Genort des Dopamin2-Rezeptors, z. B. ob das Allel A1 oder A2 vorliegt (Cinciripini et al., 2004).

Weitere wichtige Aspekte bei der Frage, welche Faktoren beim Rückfall in die Nikotinsucht eine Rolle spielen, sind psychologischer Natur. Erlernte Muster aus dem Bereich der Lerntheorie mit ihren positiven und negativen Verstärkern tragen dazu bei, den Nikotinkonsum aufrecht zu erhalten. Einen hohen Stellenwert erlangen gerade die sekundären Verstärker wie Anblick, Geruch und Geschmack des Tabaks, die z. B. durch eine Nikotinsubstitution nicht in Erscheinung treten (Rist und Watzl, 1999). Die Psychologie stellt eine Reihe von Rückfallmodellen zur Verfügung, die neben der lernpsychologischen Theorie ihre Daseinsberechtigung haben.

\subsection{Schlaf}

„Schlaf ist ein periodisch auftretender Zustand körperlicher Ruhe mit eingeschränktem Bewusstsein und minimaler Wahrnehmung der Außenwelt“ (Borbély, 2004, Seite 1), so eine mögliche Definition des Begriffs „Schlaf“. Die objektive Messung des Schlafs mithilfe der Polysomnografie (PSG) ist seit vielen Jahrzehnten möglich und die Unterscheidung von einzelnen Schlafstadien bekannt. So kann der Schlaf durch bestimmte messbare Charakteristika in Non-Rapid-Eye-Movement (NREM)und Rapid-Eye-Movement (REM)-Schlaf unterteilt werden, wobei der NREM-Schlaf als orthodoxer Schlaf bezeichnet und noch weiter in vier Stadien unterteilt wird. Die NREM-Schlafstadien 3 und 4 werden auch Tiefschlafstadien genannt. Der REM-Schlaf trägt seinen Namen aufgrund der in 
diesem Schlafstadium auftretenden schnellen Augenbewegungen und wird als paradoxer Schlaf bezeichnet, da während des Schlafens eine hohe kortikale Aktivität vorliegt, dem Wachzustand ähnlich. Diese Aktivität erleben wir als Träumen.

Die Definitionen und Charakteristika der einzelnen Schlafstadien und die Technik der PSG werden in Kapitel 2 ab Seite 29 behandelt.

\subsubsection{Schlafprofil}

Die Ergebnisse einer PSG können als Schlafprofil (Hypnogramm) dargestellt werden. Hierbei handelt es sich um die grafische Darstellung der ausgewerteten PSG-Parameter. Die X-Achse zeigt den zeitlichen Verlauf, auf der Y-Achse sind die Schlafstadien verzeichnet. Das typische Schlafprofil einer gesunden Versuchsperson ist in Abbildung $1.1 \mathrm{zu}$ finden. Aus der Abbildung ist ersichtlich, dass der normale Schlaf bestimmten Mustern folgt. So können drei bis fünf Schlafzyklen von 90- bis 120-minütiger Dauer identifiziert werden, in denen alle Schlafstadien durchlaufen werden. Nach der Wachphase finden sich nacheinander die NREM-Schlafstadien 1-4, gefolgt vom REM-Schlaf. Ebenso ist zu erkennen, dass der Tiefschlafanteil in der ersten Nachthälfte dominiert, in der zweiten jedoch vermehrt REM-Schlaf und die NREM-Schlafstadien 1 und 2 auftreten.

\subsubsection{Physiologische Funktion}

Etwa $30 \%$ unseres Lebens verbringen wir schlafend - ein 80-jähriger Mensch hat demnach theoretisch mit einer durchschnittlich achtstündigen Schlafphase etwa 27 Jahre seines Lebens geschlafen. Doch welche Funktion der Schlaf im menschlichen und tierischen Organismus erfüllt, ist weiterhin Gegenstand aktueller Forschung des noch relativ jungen Fachs Schlafmedizin. Unbestritten ist jedoch, dass Schlaf zur Erhaltung der physischen und psychischen Gesundheit essentiell ist. Im Folgenden werden einige Hypothesen zu den Funktionen des Schlafs aufgeführt.

Evolutionär betrachtet haben sich die Organismen der Erde durch die Entwicklung eines SchlafWach-Zyklus an den täglichen Tag-Nacht-Rhythmus und den damit einhergehenden Helligkeitsund Temperaturschwankungen angepasst. Um möglichst effizient den Energiehaushalt zu kontrollieren, haben sich, reguliert durch innere und äußere Zeitgeber, Ruhe- und Aktivitätsphasen 


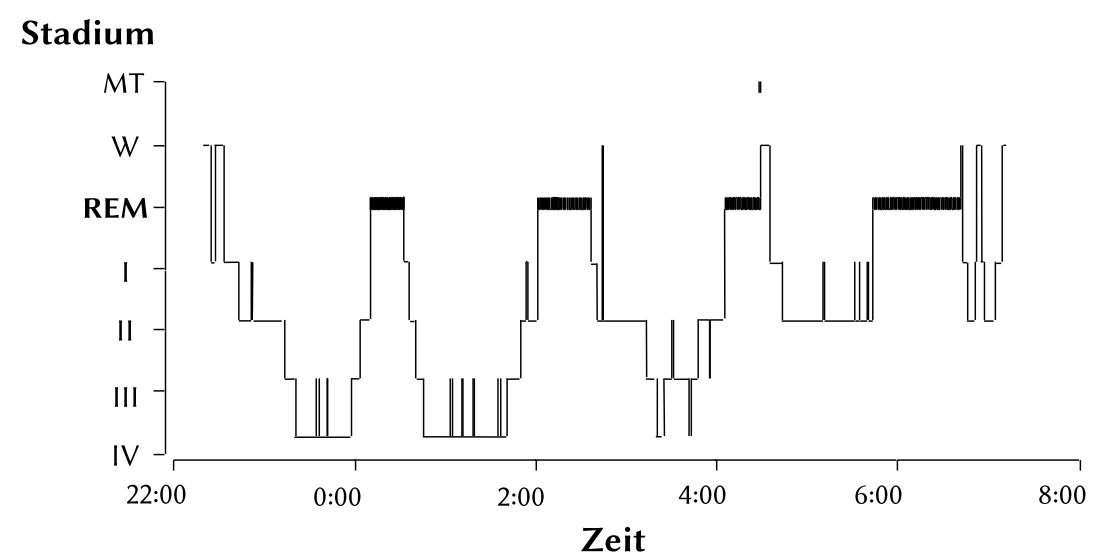

Abbildung 1.1: Schlafprofil einer gesunden Versuchsperson (MT: Movement Time, W: Wach, REM: Rapid-Eye-Movement, 1-4: Non-REM).

mit ihren charakteristischen metabolischen Aktivitätsgraden entwickelt (Schläfke und Schäfer, 1999).

Eine Hypothese zur Funktion des Schlafs ist die der Energiekonservierung. Ein ruhender Organismus verbraucht weniger Energie als ein wacher (Jung et al., 2011). Die Energie, die für das eigene Überleben und die Aufrechterhaltung der Art benötigt wird (Nahrungsaufnahme, Fortpflanzung), kann in der Aktivitätsphase effizient genutzt werden. Einige Wissenschaftler ordnen diese Energiesparmaßnahme bestimmten Schlafphasen zu. So teilt J. Horne den Schlaf in Phasen von Kern- und Füllschlaf ein und weist letzterem die Funktion der Energiekonservierung zu (Koella, 1988).

Eine andere These zu der Funktion des Schlafs ist die der Restauration. Der Schlaf stelle eine Phase der physischen und psychischen Erholung dar. Eine Theorie geht davon aus, dass den unterschiedlichen Schlafstadien bestimmte Teilbereiche der lebenserhaltenen, regenerierenden Prozesse zugeordnet sind. So sei der NREM-Schlaf für die Regenerierung der peripheren Körperfunktionen verantwortlich, der REM-Schlaf hingegen für die der zentralen (Pollmächer und Lauer, 1992). Für diese These spricht z. B. die vermehrte Ausschüttung des Wachstumshormons im Tiefschlaf, was zu einer erhöhten Gewebeneubildung führt. Gewebe wird ersetzt, akkumulierte Stoffwechselprodukte werden eliminiert (Koella, 1988). Im Sinne einer psychischen Erholung soll die gedankliche Informationsverarbeitung von großer Bedeutung sein. Hierbei erholen sich die Gehirnfunktionen, insbesondere im REM-Schlaf (Volk, 1995). Auch der Traum soll eine regenerierende Rolle spielen als eine Art „psychische Entschlackung“ (Koella, 1988). Andere Hypothesen schreiben auch dem 
NREM-Schlaf zentrale restaurative Funktionen zu, jedoch anderer Art als die des REM-Schlafs. So wird dem NREM-Schlaf die gedankliche Festigung bewusster Fakten und Ereignisse (deklarative Gedächtnisinhalte) zugeschrieben, dem REM-Schlaf hingegen die Konsolidierung unbewusster Gedanken (nicht-deklaratives Gedächtnis) (Born et al., 2006). Zudem sei der REM-Schlaf neben der Verarbeitung und Speicherung von Informationen dafür verantwortlich, dass Gedankeninhalte des Kurzzeitgedächtnisses in das Langzeitgedächtnis übergehen und weniger wichtige Informationen gelöscht werden (Volk, 1995).

Neben diesen Hypothesen scheint der REM-Schlaf auch eine wichtige Rolle in der Entwicklung und Reifung des ZNS zu spielen. Ein Neugeborenes weist einen REM-Schlafanteil von über $50 \%$ des Gesamtschlafs auf, ein Erwachsener hingegen nur circa 18 \% (Pollmächer und Lauer, 1992). Die neuronalen Netzwerke profitieren anscheinend von der Aktivierung im REM-Schlaf und das Wachstum von Axonen, Dendriten und Synapsen wird gefördert.

\subsubsection{Regulation}

Wie alle körperlichen Vorgänge unterliegen auch Schlafen und Wachen einer zentralnervösen Steuerung. Bezüglich dieser Regulation existiert eine Vielzahl von Theorien und Hypothesen, von denen sich einige in der Schlafforschung durchgesetzt haben, für deren Richtigkeit aber ein endgültiger Beweis bis heute fehlt. Im Folgenden werden die aktuellen Theorien zur Schlafregulation dargestellt.

Die Schlafregulation lässt sich in zwei Teilbereiche gliedern. Zum einen in die Schlaf-WachRegulation, zum anderen in die interne Schlafregulation. Hierbei steuert die Schlaf-Wach-Regulation u. a., wann und wie lange ein Individuum schläft, die interne Schlafregulation hingegen die zeitliche Abfolge der Schlafstadien, also die zyklische Schlafstruktur (Pollmächer und Lauer, 1992).

Schlaf-Wach-Regulation Die Schlaf-Wach-Regulation besitzt zwei Teilaspekte, die homöostatische und periodische Komponente. Beide werden durch äußere Faktoren, wie z. B. soziale Kontakte, Umgebung oder Tätigkeit, beeinflusst. Beide Komponenten bilden zusammen das „ZweiProzess- oder Schwellenmodell“, erstmalig von Borbély (1982) beschrieben und von Daan et al. 
(1984) erweitert. Hierbei steht die homöostatische Komponente für eine Art Schlafgedächtnis, welches speichert, wie lange das Individuum wach ist. Je länger dies der Fall ist, desto höher steigt der sogenannte Schlafdruck. Im Zwei-Prozess-Modell wird die homöostatische Komponente als Prozess S bezeichnet und als sein polysomnografisches Korrelat der Tiefschlaf angenommen, da nach dieser Theorie der Schlafdruck im Tiefschlaf abgebaut wird. Ein Argument hierfür bietet die Tatsache, dass die vorausgegangene Wachzeit positiv mit der Tiefschlafdauer korreliert. Die periodische Komponente steht für sämtliche zyklische Abläufe im Organismus, die durch interne Zeitgeber generiert werden. Als anatomisches Korrelat einer solchen inneren Uhr wird der Nucleus suprachiasmaticus (SCN) angesehen. Dieser erhält u. a. über Afferenzen aus der Retina Informationen über die Helligkeit der Umgebung und synchronisiert so ständig seinen eigenen zirkadianen Rhythmus mit der Außenwelt. Efferent innerviert der SCN u. a. Hirnregionen, die für die Regulation des Blutdrucks, der Körpertemperatur oder Hormonausschüttung zuständig sind. So folgt z. B. die Körpertemperatur oder die Hormonkonzentration von Cortisol oder Melatonin gewissen zeitlichen Mustern. Im Zwei-Prozess-Modell wird diese zirkadiane Periodik als Prozess C bezeichnet (Rodenbeck, 2011; Pollmächer und Lauer, 1992). Die Interaktion beider Komponenten ist in Abbildung 1.2 ersichtlich.

Der Prozess S, in Abbildung 1.2 als durchgezogene Linie erkennbar, steigt im Laufe des Tages als Ausdruck für den Schlafdruck an, da sich die Wachperiode stetig verlängert. Im Schlaf wird das Schlafbedürfnis abgebaut und die Kurve fällt relativ steil ab. Der Prozess C hingegen, der die zirkadianen Abläufe repräsentiert, hat einen anderen Verlauf und wird in Abbildung 1.2 als zwei wellenförmige Kurven (gestrichelte Linien) dargestellt. Beide Prozesse bestimmen durch

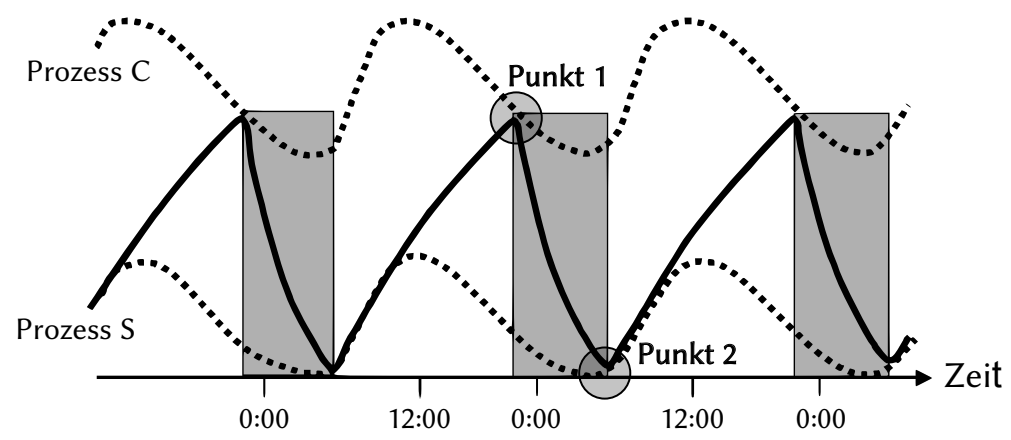

Abbildung 1.2: Das Zwei-Prozess-Modell. Die graue Fläche stellt die Schlafphase dar. Modifiziert nach Rodenbeck, 2011, Seite 1272. 
ihren Kurvenverlauf und die hierdurch resultierenden Berührungspunkte die Zeitpunkte des Einschlafens und Aufwachens. So ist bei Punkt 1 in Abbildung 1.2 die Wahrscheinlichkeit des Einschlafens im Tagesverlauf am höchsten, da hier die Wachperiode schon lange andauert und die zirkadianen Prozesse mit z. B. niedriger Körpertemperatur oder niedrigem Blut-Cortisolspiegel den Schlaf fördern werden. Bei Punkt 2 hingegen sorgt der Prozess C mit z. B. ansteigendem Blut-Cortisolspiegel und Prozess S mit geringem Schlafdruck für ein Erwachen. Durch diese beiden Berührungspunkte sind sogenannte Einschlaf- und Aufwachschwellen definiert, weshalb das ZweiProzess-Modell auch Zwei-Schwellen-Modell genannt wird (Rodenbeck, 2011; Pollmächer und Lauer, 1992).

Neurochemisch und elektrophysiologisch werden Schlafen und Wachen durch die verschiedensten Hirnareale reguliert, darunter das aufsteigende retikuläre Aktivierungssystem (ARAS) der Formatio reticularis des Hirnstamms, der Thalamus und Hypothalamus sowie der Kortex (Happe und Walther, 2009). Im Sinne eines Flip-Flop-Mechanismus (Saper et al., 2001) interagieren diese Hirnregionen indem sie abwechselnd Schlaf fördern und Wachheit unterdrücken und umgekehrt. So fördern z. B. die monoaminergen Neurone des Locus coeruleus und der dorsalen Raphekerne durch Erregung des Kortex und Hemmung der schlaffördernden Neurone des Nucleus präopticus venterolateralis (VLPO) des Hypothalamus die Wachheit. Im Schlaf hingegen werden die monoaminergen Neurone von denen des VLPOs gehemmt. Dieses System wird durch den modulierenden Einfluss des Neuropeptids Orexin (oder Hypocretin) stabilisiert (Rodenbeck, 2011; Saper et al., 2005).

Interne Schlafregulation Die interne Schlafregulation, die die Schlafstruktur mit dem Wechsel zwischen REM- und NREM-Schlaf bestimmt, ist von McCarley und Hobson (1975) in dem sogenannten reziproken Interaktionsmodell beschrieben worden. Bis heute hat eine revidierte Form dieser Theorie Bestand. Sie besagt, dass das Zusammenspiel aminerger und cholinerger Neurone den zyklischen Wechsel von REM- und NREM-Schlaf bewirkt. Die Nervenzellen des Locus coeruleus und die der Raphekerne werden als „REM-off“-Neurone bezeichnet und hemmen mit den Neurotransmittern Noradrenalin bzw. Serotonin die cholinergen Nervenzellen der Brückenhaube. Die Neuronen der Brückenhaube hingegen werden „REM-on“-Neurone genannt und hemmen die 
aminergen REM-off-Neurone. Da all diese Neurone auch autoinhibitorisch wirken, entsteht eine wellenförmige reziproke Hemmung (Rodenbeck, 2011; Pollmächer und Lauer, 1992). Abbildung 1.3 zeigt das reziproke Interaktionsmodell.

Es entsteht durch eine hohe aminerge Hemmung durch die REM-off-Neurone eine NREM-Phase. Da diese Hemmung aber im Laufe der Zeit durch die Autoinhibition an Intensität verliert, überwiegt zunehmend die cholinerge Aktivität der REM-on-Neurone. Die dadurch bewirkte Hemmung der aminergen Neurone führt zu einer REM-Phase. Da auch diese Hemmung ihre Intensität verliert, wird wieder eine NREM-Phase eingeleitet. Dieser Vorgang wiederholt sich im Laufe des Schlafens alle 90-120 Minuten, so dass die typische Schlafarchitektur entsteht (Rodenbeck, 2011; Pollmächer und Lauer, 1992).

Durch den beschriebenen Mechanismus ist also ein Rückschluss von Schlafstadien auf die vorherrschenden Transmittersysteme möglich. Der NREM-Schlaf wird vom aminergen System dominiert, der REM-Schlaf hingegen vom cholinergen System. So kann beispielsweise die bei Depressionen vorliegende verkürzte REM-Latenz (siehe 4.3 auf Seite 88) durch eine erhöhte cholinerge Aktivität erklärt werden. Abbildung 1.4 verdeutlicht den Zusammenhang.

\subsection{Dopamin}

Der Botenstoff Dopamin gehört als Catecholamin zu der Gruppe der biogenen Amine. Er fungiert im Organismus als Neurotransmitter und wird in den Zellen des Nebennierenmarks und des ZNS

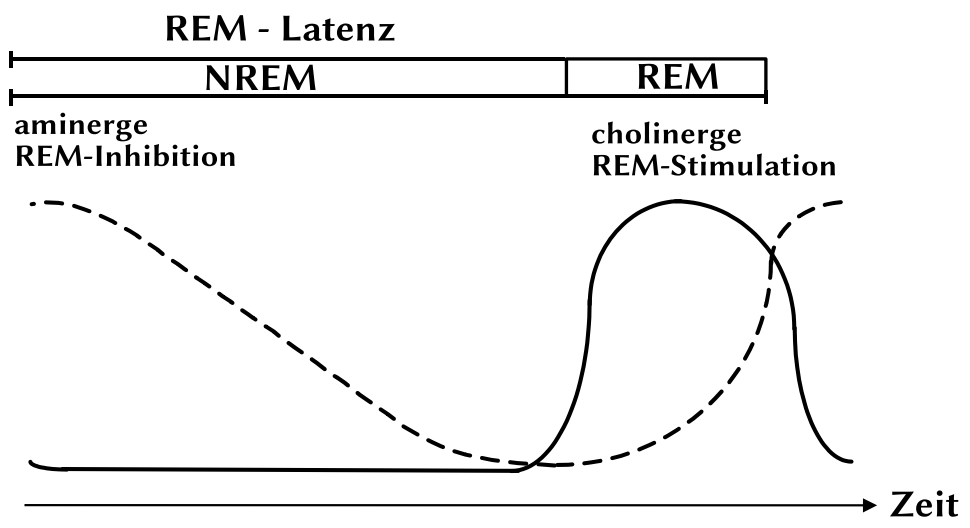

Abbildung 1.3: Reziprokes Interaktionsmodell. Modifiziert nach Rodenbeck, 2011, Seite 1272. 


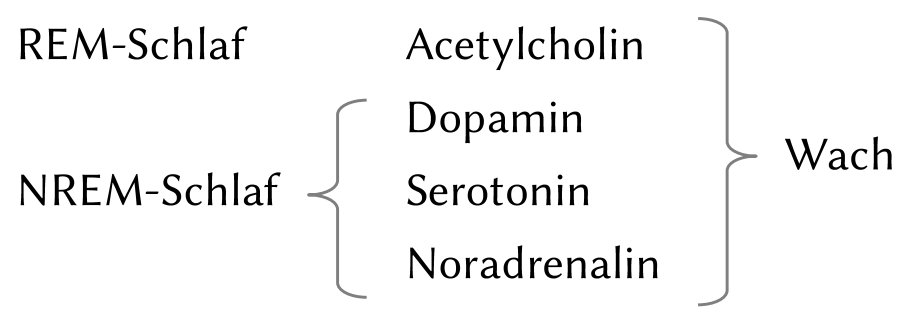

Abbildung 1.4: Polysomnografische Stadien und zugehörige Transmitter.

aus der Aminosäure Tyrosin synthetisiert. Betrachtet man das ZNS, finden sich dopaminerge Nervenzellen u. a. in der Substantia nigra und anderen Bereichen des Mesencephalons und im Diencephalon. Funktionell sind diese Bereiche mit dem Bewegungsantrieb (Substantia nigra), der Regulation vegetativer Vorgänge (Diencephalon) und der Beeinflussung psychischer und verhaltensbiologischer Abläufe (andere Zellgruppen des Mesencephalons) verknüpft. Neuronale Bahnen ziehen von diesen dopaminergen Zellgruppen in viele Teile des Gehirns, darunter die Basalganglien, das limbische System und der Neokortex.

Die Wirkungen von Dopamin werden über Dopaminrezeptoren vermittelt. Zurzeit unterscheidet man zwei Dopaminrezeptorklassen (D1- und D2-artige Rezeptoren). Zu der Gruppe der D1-artigen Rezeptoren zählen die D1- und D5-Rezeptoren, zu der Gruppe der D2-artigen Rezeptoren die D2-, D3- und D4-Rezeptoren. Die Dopaminrezeptoren kommen prä- wie postsynaptisch vor und besitzen je nach Rezeptortyp und -lage unterschiedliche Funktionen.

Der Zusammenhang zwischen Dopamin und den periodischen Beinbewegungen (Periodic-LegMovements, PLM), beispielsweise im Rahmen des Syndroms der unruhigen Beine (Restless-LegsSyndrom, RLS), wird in 1.6.2 auf Seite 26 näher beschrieben.

\subsubsection{Das Belohnungssystem}

Eine wichtige Funktion spielt Dopamin, umgangssprachlich als Glückshormon bezeichnet, im Belohnungssystem des Gehirns. Dies ist phylogenetisch betrachtet ein sehr alter Teil des ZNS. Es sorgt mithilfe der positiven und negativen Verstärkung von Verhaltensweisen dafür, dass das Überleben der eigenen Art gesichert ist. So stellt sich durch ein bestimmtes Verhalten wie Nahrungs- und Flüssigkeitsaufnahme, sexuelle Aktivität oder elterliche Fürsorge eine Befriedigung 
(primäre Verstärkung) ein, die zu einer Wiederholung des Verhaltens führt. Das Individuum lernt, physiologische Bedürfnisse zu stillen, und sorgt somit gleichzeitig für das Fortbestehen der Art (Heinz und Batra, 2003).

Strukturell werden dem Belohnungssystem, das auch mesolimbisches dopaminerges Verstärkungssystem genannt wird, einige aufsteigende Nervenbahnen des Mittelhirns zugerechnet. Diese Bahnen entspringen z. B. in der Substantia nigra oder dem seitlichen Tegmentalgebiet und ziehen in diverse Hirnregionen, darunter die Basalganglien (insbesondere Nucleus accumbens), das limbische System und der Kortex. Eine Vielzahl an hemmenden und erregenden Nervenbahnen aus vielen verschiedenen Hirnregionen beeinflusst das System mit den unterschiedlichsten Neurotransmittern. Die Botenstoffe dieses Systems sind u. a. Endorphine, Gamma-Aminobuttersäure (GABA), Glutamat und Dopamin. Letzteres ist der prominenteste Transmitter und Namensgeber des Belohnungssystems, in welchem die Botenstoffe bei einer Aktivierung vermehrt ausgeschüttet oder gehemmt werden.

\subsection{Hypothalamus-Hypophysen-Nebennieren-Achse}

Als Hypothalamus-Hypophysen-Nebennieren-Achse (HHN-Achse) bezeichnet man die Hormonkaskade, die zwischen dem Hypothalamus, der Hypophyse und den Nebennieren als Kommunikationsweg dient.

Regulation Gesteuert wird diese Achse durch äußere und innere Faktoren wie die zirkadiane Rhythmik, körperliche und seelische Stresszustände oder die Plasmakonzentrationen der beteiligten Hormone Corticotropin-Releasinghormon (CRH), Adrenocorticotropes Hormon (ACTH) und die in der Nebennierenrinde produzierten Steroidhormone. Die jeweils übergeordnete Struktur fördert durch Hormonausschüttung die Freisetzung von Hormonen aus der untergeordneten Struktur. Hierarchisch betrachtet folgt auf den Hypothalamus (CRH) die Hypophyse (ACTH) und die Nebenniere (Steroidhormone). Reguliert werden die Hormonkonzentrationen zum einen über die Förderung einer Hormonausschüttung durch die an der HHN-Achse beteiligten Strukturen und zum anderen über eine negative Rückkopplung. Das bedeutet, dass die Plasmakonzentrationen der 
Hormone der untergeordneten Strukturen die Ausschüttung von Hormonen der übergeordneten Strukturen hemmen. Die Ausschüttung der beteiligten Hormone folgt pulsatil einer zirkadianen Rhythmik mit einem Maximum in den Morgenstunden und einem Tiefpunkt am Abend.

Funktion Die Funktion der HHN-Achse besteht in der Aufrechterhaltung von wichtigen Lebensfunktionen sowie in der Ermöglichung einer adäquaten Reaktion auf Stress. So steuert dieses System u. a. den Energiehaushalt, Immunfunktionen, psychische Funktionen und Sexualität und ist somit für die Homöostase und Koordination dieser Körperfunktionen unerlässlich. Im Falle von körperlichen und/oder seelischen Stresszuständen wird die zirkadiane Rhythmik durchbrochen und es kommt neben einer Aktivierung des Sympathikus und einer vermehrten Ausschüttung von Vasopressin zur Sekretion von CRH. Diese drei Faktoren führen zu einer vermehrten Freisetzung von ACTH und somit u. a. zur Ausschüttung von Cortisol, welches für die schnelle Bereitstellung von Energie und zur Modulation des Immunsystems benötigt wird. Als Endprodukte der HHN-Achse werden in der Nebennierenrinde Steroidhormone, die sogenannten Corticosteroide, synthetisiert, zu denen die Mineralocorticoide, Glucocorticoide und Androgene gehören.

Diese Übersichtsarbeit konzentriert sich auf das Glucocorticoid Cortisol, welches als Teil der HHN-Achse zahlreiche Funktionen übernimmt und im Folgenden genauer dargestellt wird.

\subsubsection{Cortisol}

Biosynthese Cortisol (oder Hydrocortison) ist ein Steroidhormon und wird in der Nebennierenrinde aus Cholesterol synthetisiert. Es gehört zu der Gruppe der Glucocorticoide. ACTH induziert die Bildung der Enzyme, die für die Synthese von Cortisol notwendig sind. Zudem sorgt es für den Einbau von Low-Density-Lipoprotein-Rezeptoren (LDL-Rezeptoren) in die Zellwand der Nebennierenzellen, um Cholesterol für die Synthese zur Verfügung zu stellen.

Transport Im Blut findet man freies und an Transportproteine gebundenes Cortisol und Cortisolmetaboliten. Das freie Cortisol ist das physiologisch aktive Hormon und macht einen Anteil von weniger als $5 \%$ des im Blut zirkulierenden Cortisols aus. Transportproteine wie das cortisolbindende Globulin (CBG) oder Albumin verbessern die Löslichkeit des Steroids. 
Rezeptoren Die Glucocorticoide entfalten ihr Wirkung über zytoplasmatische Rezeptorproteine, die ihrerseits die Transkription bestimmter Gene beeinflussen und somit eine Zellantwort auslösen. Diese Glucocorticoid-Rezeptoren befinden sich in fast allen Organen, was ihre vielfältigen Wirkungen erklärt.

Abbau Der Abbau von Cortisol erfolgt durch Biotransformation in der Leber. Es wird mit Glucuronsäure oder Schwefelsäure konjugiert und mit dem Harn und der Galle ausgeschieden.

Physiologische Wirkungen Cortisol hat vielfältige Aufgaben im Organismus. Es greift regulierend in den Intermediärstoffwechsel und in das Immunsystem ein. Hiermit spielt es eine entscheidende Rolle in der Stressbewältigung, z. B. nach schweren Traumata. Es stellt durch seine katabole Wirkung innerhalb von Minuten energiereiche Stoffwechselprodukte zur Verfügung und reduziert andere, nicht benötigte metabolische Aktivitäten. Es reguliert somit den Protein-, Fettund Kohlenhydratstoffwechsel, sorgt als Insulinantagonist und Förderer der Gluconeogenese für einen Anstieg des Blutzuckerspiegels und mobilisiert Fettsäuren und glycogene Aminosäuren. Durch eine verstärkte Catecholaminwirkung steigt durch Cortisol die Herzkraft und die Gefäße verengen sich.

Cortisol wird neben den Catecholaminen zu den Stresshormonen gezählt und die HHN-Achse wird auch als „Stressachse“ bezeichnet. Die Aktivität dieser Achse kann bei chronischen Stresszuständen (auch psychischer Natur) dauerhaft verändert sein. Dabei können die Hormone der HHN-Achse in zu hohen oder zu niedrigen Blutkonzentrationen vorliegen.

Neben diesen Effekten haben Glucocorticoide wie Cortisol auch Einfluss auf den Wasserhaushalt. Sie hemmen die Vasopressin-Sekretion und erhöhen die glomeruläre Filtrationsrate. Hohe Cortisolspiegel bewirken hingegen mineralocorticoide Effekte und sorgen hierdurch für eine geringere Wasserausscheidung des Organismus.

Cortisol besitzt antiinflammatorische Eigenschaften und wirkt immunsuppressiv. Es hemmt u. a. Entzündungsmediatoren, reduziert die Anzahl verfügbarer T-Zellen und Eosinophilen und beeinflusst die Mikrovaskularisation. Zudem hat Cortisol eine antipyretische Wirkung und hemmt die Bildung von Prostaglandinen. Aufgrund der antiinflammatorischen, immunsuppressiven und 
zytostatischen Wirkungen der Glucocorticoide werden diese in der Therapie verschiedenster Krankheiten eingesetzt, darunter rheumatische Erkrankungen, Allergien, Autoimmun- und Krebserkrankungen. Zudem werden sie zur Substitution bei Cortisolmangelerkrankungen benötigt.

Neben diesen körperlichen Wirkungen besitzt Cortisol auch einen Einfluss auf psychische Funktionen. Dieser umfasst Verhalten und die psychische Befindlichkeit, besonders zu beobachten bei Cortisolüberschuss (z. B. beim Cushing-Syndrom: Gefühlslabilität, Depression, Verwirrtheit, Psychosen) oder -mangel (z. B. beim Addison-Syndrom: Persönlichkeitsveränderungen, Unruhe, Reizbarkeit).

\subsection{Der Zusammenhang zwischen Nikotin, Schlaf, Dopamin und HHN-Achse}

\subsubsection{Nikotin und Schlaf}

Die Forschung geht davon aus, dass sowohl der Konsum von Nikotin als auch ein Nikotinentzug Schlafstörungen verursachen können. Schlafstörungen wiederum, verbunden mit den Folgen wie Tagesmüdigkeit und Depressionen, fördern die Aufrechterhaltung der Abhängigkeit und können eine erfolgreiche Abstinenz verhindern. Es wird angenommen, dass Nikotin den Schlaf über die Ausschüttung diverser Neurotransmitter beeinflusst, die an der Regulation des Schlafs beteiligt sind. Beispielsweise fördert Nikotin die Freisetzung von Acetylcholin (Benowitz, 2008a), welches als vorherrschender Transmitter in der Generierung des REM-Schlafs gilt. Auch die vermehrte Ausschüttung von Dopamin durch Nikotin führt - verbunden mit einer Aktivierung des Belohnungssystems - zu einer erhöhten Wachsamkeit und damit zu einem gestörten Schlaf. Tierexperimentell konnte gezeigt werden, dass Nikotinrezeptoren an der Steuerung des Schlafs beteiligt sind (Léna et al., 2004) und Nikotin den Schlaf beeinflussen kann (z. B in Salín-Pascual et al., 1999). 


\subsubsection{Nikotin und Dopamin}

Das Belohnungssystem Nikotin greift in die unterschiedlichsten Transmittersysteme ein. Es ist in der Lage, die Freisetzung von Dopamin zu stimulieren (Benowitz, 2008a). Nikotin besitzt hierdurch die Fähigkeit, über eine Aktivierung des Belohnungssystems des ZNS eine Abhängigkeit zu erzeugen. Über die Bindung von Nikotin an die nACh-Rezeptoren, die sich auf Zellen des Belohnungssystems (z. B. auf Zellen des seitlichen Tegmentalgebiets) befinden, wird Dopamin ausgeschüttet, u. a. in den Nucleus accumbens (Brody, 2006). Hierbei ist besonders eine Untereinheit des Nikotinrezeptors von Bedeutung: Die $\beta 2$-Untereinheit ist in die Freisetzung von Dopamin involviert und sorgt hiermit bei Nikotinkonsum für die Aufrechterhaltung der Abhängigkeit (Benowitz, 2008a). Dies konnte durch Studien mit genetisch veränderten Mäusen festgestellt werden: Fehlte den Mäusen die $\beta 2$-Untereinheit im Nikotinrezeptor ( $\beta 2$-nACh-Rezeptor-Knockout), konnte der bei den Wildtyp-Mäusen nach Nikotingabe beobachtete dosisabhängige Anstieg der Dopaminkonzentration nicht beobachtet werden (Maskos et al., 2006).

Die Ausschüttung von Dopamin soll für die Wirkungen des Nikotins verantwortlich sein, die einen weiteren Konsum fördern. Die erhöhten Dopaminlevel werden als Lustempfinden, Genuss und Glücksgefühl wahrgenommen und als solche im Gedächtnis behalten. Neben den angeborenen primären Verstärkern aktiviert also auch der Konsum von Tabakprodukten das Belohnungssystem. Die Verstärkung durch das Belohnungssystem ist eine Voraussetzung für die Entwicklung eines abhängigen Verhaltens. Gleichermaßen wie z. B. die Nahrungsaufnahme wird der Tabakkonsum positiv (durch die angenehmen Wirkungen) und negativ (durch den Wegfall von aversiven Zuständen) verstärkt. Das Verhalten, hier der Konsum von Tabak, wird häufiger gezeigt.

Eine weitere Ebene kommt durch die sekundäre Verstärkung hinzu, die dadurch gekennzeichnet ist, dass ehemals neutrale Reize ebenfalls eine positive, mit dem Verhalten verknüpfte Assoziation erwerben. Dies kann z. B. der Anblick oder der Geruch von Zigaretten sein, der nun selbst eine Aktivierung des Belohnungssystems auslöst (Rist und Watzl, 1999). Man nimmt an, dass es im Falle eines Entzugs durch die geringere Freisetzung von Dopamin zu einem hypodopaminergen Zustand kommt, der u. a. verantwortlich ist für die Entzugssymptomatik. Die Ansprechbarkeit des Belohnungssystems auf andere Stimuli als das Rauchen soll vermindert sein und trägt dazu bei, 
dass weniger Freude empfunden wird (Benowitz, 2008a).

Nikotin, Dopamin und Schlaf Die Freisetzung von Dopamin durch Nikotin hat einen Einfluss auf den Schlaf (s. o.). Diesbezüglich scheint das dopaminerge System noch auf einer anderen Ebene mit Nikotin verbunden zu sein. Das Restless-Legs-Syndrom (RLS), häufig verbunden mit periodischen Beinbewegungen (Periodic-Leg-Movements, PLM) stellt eine Erkrankung dar, die mit dem Rauchen assoziiert ist (Ohayon und Roth, 2002). Die Pathophysiologie dieser chronischen Krankheit ist unbekannt, jedoch geht man von einer Beteiligung des dopaminergen und opioidergen Systems aus. Für diese These spricht das gute Ansprechen der Erkrankung auf Levodopa, Dopaminagonisten und Opioide (Wetter und Pollmächer, 1997). Auch die Beobachtung passagerer, funktioneller Veränderungen im dopaminergen System (u. a. grenzwertig erniedrigte dopaminerge striatale Rezeptorbindungen) sprechen für diese Annahme (Trenkwalder, 2007). Charakteristisch ist u. a. der bei den Betroffenen gestörte Schlaf, der sich in einer stärkeren Fragmentierung des Schlafprofils widerspiegelt. Ob der Tabakkonsum die Entstehung einer RLS-Erkrankung begünstigt oder Raucher eine bestehende RLS-Erkrankung durch den Konsum von Tabak als eine Art Selbstmedikation therapieren, ist nicht bekannt. Letzteres wäre denkbar, wenn man davon ausgeht, dass Nikotin den bei RLS-Patienten erniedrigten Dopaminspiegel anhebt. Die somit erhöhte Konzentration an Dopamin könnte die Symptome bessern.

Inwieweit sich die PLM auf den Erfolg eines Entzugs auswirken können, wurde für die Alkoholabhängigkeit bereits gezeigt. Hier war ein erhöhter PLM-Index mit einem größeren Rückfallrisiko verbunden (Gann et al., 2002). Überträgt man diese Beobachtung auf die Nikotinabhängigkeit, könnten die im Entzug auftretenden Schlafstörungen auch durch die vermehrten PLM erklärt werden.

\subsubsection{Nikotin und HHN-Achse}

Nikotin stimuliert die Freisetzung von Stresshormonen der HHN-Achse wie beispielsweise Cortisol (z. B. in Newhouse et al., 1990). Inwieweit die Cortisolkonzentration oder die Funktion der HHNAchse bei abhängigen Rauchern verändert ist, wird kontrovers diskutiert. Für einen Entzug wird jedoch angenommen, dass die Hormonspiegel der Stresshormone langfristig durch den Wegfall 
der stimulierenden Wirkung von Nikotin sinken (z. B. in Puddey et al., 1984). Allerdings bedeutet ein akuter Entzug für Körper und Psyche Stress, was möglicherweise mit einer Aktivierung der HHN-Achse einhergeht.

Nikotin, HHN-Achse und Schlaf Die enge Verbindung zwischen den zyklischen Mustern der Cortisolsekretion und des Schlafens und Wachens und die Beeinflussung beider Systeme durch Nikotin rechtfertigt eine gemeinsame Betrachtung. Cortisol, welches entscheidend in den Energiehaushalt und den Stoffwechsel eingreift, wird insbesondere in der Aufwachphase ausgeschüttet und fördert den Wachzustand. Demnach ist denkbar, dass Nikotin den Schlaf auch über eine vermehrte Sekretion von Cortisol stört. Ein Zusammenhang zwischen einer erhöhten Cortisolkonzentration und gestörtem Schlaf ist bereits gezeigt worden (Rodenbeck et al., 2002).

\subsection{Fragestellung und Zielsetzung}

Die vorliegende Arbeit hat das Ziel, durch eine systematische Literaturrecherche Studien zu finden, die sich mit den Auswirkungen eines Nikotinkonsums und eines Nikotinentzugs auf den Schlaf und endokrine Parameter der HHN-Achse und des dopaminergen Systems beschäftigt haben. Es soll ein bewertender Überblick über die bisher zu diesem Thema veröffentlichten Studien dargestellt werden, der die Forschungsergebnisse zusammenfasst und in Relation zueinander setzt. Es wird versucht, aus den Studienergebnissen Faktoren zu ermitteln, die eine hohe Vorhersagekraft für einen Rückfall in die Nikotinabhängigkeit besitzen. Mit dem Wissen um geeignete Prädiktoren für einen Rückfall und geeigneten Maßnahmen könnten möglicherweise die Abstinenzraten zukünftig erhöht werden. Eine weiteres Ziel der vorliegenden Arbeit ist es zu beurteilen, ob die Erfassung von Schlaf- und endokrinen Parametern sinnvoll ist, um einen Entzug objektiv zu bewerten, und ob Korrelationen von objektiven und subjektiven Messergebnissen im Entzug möglich sind.

Obwohl bereits eine Vielzahl von Studien veröffentlicht wurde, die die Zusammenhänge zwischen Nikotin, Endokrinum und Schlaf untersucht haben, bleiben offene Fragen. Die vorliegende Arbeit soll auch auf die Lücken der Forschung hinweisen und darlegen, an welcher Stelle noch 
Forschungsbedarf besteht. Die konkreten Fragen, die durch eine systematische Literaturrecherche und die Diskussion der Ergebnisse beantwortet werden sollen, lauten:

- Welche Auswirkungen auf objektive Schlafparameter und die Hormone der HHN-Achse hat die Gabe von Nikotin in tierexperimentellen Untersuchungen?

- Welche Auswirkungen auf objektive Schlafparameter, das dopaminerge System und die Hormone der HHN-Achse hat die Gabe von Nikotin an Nichtraucher?

- Welche Auswirkungen hat ein Nikotinentzug bei Gelegenheitsrauchern und Rauchern auf objektive Schlafparameter, das dopaminerge System und die Hormone der HHN-Achse?

- Wie unterscheiden sich die objektiven Schlafparameter, das dopaminerge System und die Hormone der HHN-Achse zwischen Nichtrauchern nach Nikotingabe und Gelegenheitsrauchern und Rauchern?

- Wie unterscheiden sich die objektiven Schlafparameter, das dopaminerge System und die Hormone der HHN-Achse zwischen Gelegenheitsrauchern und Rauchern im Entzug?

- Können aus den Studienergebnissen Faktoren ermittelt werden, die eine hohe Vorhersagekraft bezüglich eines möglichen Rückfalls in die Nikotinabhängigkeit besitzen, und was sind dies für Faktoren?

- Ist es sinnvoll, den Schlaf, die HHN-Achse oder das dopaminerge System zu beobachten, um einen Entzug objektiv zu messen, und lassen sich die objektiven Messergebnisse als neurobiologische Korrelate der subjektiven Symptome deuten?

- Wo bestehen Lücken in der Nikotinforschung? 


\section{Methoden}

\subsection{Definitionen von Rauchgewohnheiten}

Folgend sind einige Definitionen zur Einteilung von Rauchgewohnheiten aufgeführt (modifiziert nach CDC/National Center for Health Statistics, 2009).

- Niemals-Raucher: Person, die niemals oder weniger als 100 Zigaretten in ihrem Leben geraucht hat.

- Nichtraucher: Person, die zum jetzigen Zeitpunkt nicht raucht.

- Ehemaliger Raucher: Person, die mindestens 100 Zigaretten in ihrem Leben geraucht hat, aber zum jetzigen Zeitpunkt nicht mehr raucht.

- Gelegenheitsraucher: Person, die mindestens 100 Zigaretten in ihrem Leben geraucht hat und die zum jetzigen Zeitpunkt raucht, aber nicht täglich.

- Raucher: Person, die mindestens 100 Zigaretten in ihrem Leben geraucht hat und die zum jetzigen Zeitpunkt raucht.

\subsection{Polysomnografie}

Mithilfe der Elektroenzephalografie (EEG) kann der Schlaf objektiv erfasst und gemessen werden. Wird die Schlaf-EEG noch durch weitere Messverfahren ergänzt (z. B. Elektrookulografie, Elektromyografie des Mundbodens und der Beine, Elektrokardiografie, Pulsoxymetrie, Messung des Atemflusses und der Atembewegung), wird eine solche Ableitung als Polysomnografie (PSG) bezeichnet. 
Grundlage der EEG ist die Messung der bioelektrischen Aktivität des Gehirns, die durch Summation postsynaptischer Potentiale entsteht. Die auftretenden natürlichen Schwankungen dieser Potentiale werden in einem standardisierten Verfahren (10-20-System) mittels Elektroden auf der Schädelkalotte abgeleitet, wobei die zwischen zwei Elektroden ermittelte Potentialdifferenz verstärkt und aufgezeichnet wird.

Die EEG-Aktivität stellt sich als Wellen verschiedener Frequenzbereiche und Amplituden dar. Mithilfe der Auswertungskriterien nach Rechtschaffen und Kales (1968) können einzelnen Frequenzbereichen unterschiedliche Schlafstadien zugeordnet werden.

\subsection{Definitionen von Schlafvariablen}

Die Kriterien für die Zuordnung der Schlafstadien und eine Standardisierung von Aufnahmetechniken entwickelten Rechtschaffen und Kales (1968) mithilfe eines Komitees der Universität Kalifornien in Los Angeles und veröffentlichten die Ergebnisse in einem Handbuch.

Tabelle 2.1 bietet einen Überblick über die Frequenzbereiche der EEG-Wellen und die zugehörigen Stadien mit den typischen Charakteristika.

Neben den klassischen Schlafstadien können weitere Schlafparameter bestimmt werden, deren Definitionen (modifiziert nach Pollmächer und Lauer, 1992) im Folgenden aufgeführt sind. Es handelt sich um allgemeine Definitionen, die von denen in den Studien verwendeten Definitionen abweichen können.

- Gesamtschlafzeit: Gesamtzeit, die von „Licht aus“ bis „Licht an“ schlafend verbracht wurde (alle Stadien außer Wach).

- Schlaf-Latenz: Zeit zwischen „Licht aus“ und dem erstmaligen Auftreten von NREM-Schlafstadium 2.

- Schlaf-Effizienz: Prozentualer Anteil der Gesamtschlafzeit von „Licht aus“ bis „Licht an“.

- REM-Latenz: Zeit zwischen dem Einschlafen und der ersten REM-Schlafphase.

- REM-Frequenz: Anzahl an REM-Schlafphasen bezogen auf die Gesamtschlafzeit. 
Tabelle 2.1: Definitionen der polysomnografischen Stadien. Modifiziert nach Pollmächer und Lauer, 1992, Seite 11.

\begin{tabular}{|c|c|c|c|}
\hline Stadien & EEG & Charakteristika & Alternative Bezeichnung \\
\hline Wachzustand & Dominierende $\alpha$-Aktivität & $\begin{array}{l}\text { Lidschläge, rasche Augenbewegungen, hoher } \\
\text { Muskeltonus, Bewegungsartefakte }\end{array}$ & \\
\hline $\begin{array}{l}\text { NREM- } \\
\text { Schlafstadium } 1\end{array}$ & $\theta$-Aktivität, Vertexzacken & $\begin{array}{l}\text { Langsame Augenbewegungen, Abnahme des } \\
\text { Muskeltonus }\end{array}$ & $\begin{array}{l}\text { Orthodoxer Schlaf, leichter Schlaf, in } \\
\text { tierexperimentellen Studien } \\
\text { Slow-Wave-Sleep (SWS) } 1\end{array}$ \\
\hline $\begin{array}{l}\text { NREM- } \\
\text { Schlafstadium } 2\end{array}$ & $\begin{array}{l}\theta \text {-Aktivität, K-Komplexe, } \\
\text { Schlafspindeln }\end{array}$ & $\begin{array}{l}\text { Keine Augenbewegungen, EEG-Artefakte, } \\
\text { Abnahme des Muskeltonus }\end{array}$ & $\begin{array}{l}\text { Orthodoxer Schlaf, leichter Schlaf, } \\
\text { Spindelschlaf, in tierexperimentellen } \\
\text { Studien SWS } 1\end{array}$ \\
\hline $\begin{array}{l}\text { NREM- } \\
\text { Schlafstadium } 3\end{array}$ & $\begin{array}{l}>20 \text { und }<50 \% \text { hohe } \\
\delta \text {-Wellen }\end{array}$ & $\begin{array}{l}\text { Keine Augenbewegungen, EEG-Artefakte, } \\
\text { Abnahme des Muskeltonus }\end{array}$ & $\begin{array}{l}\text { Orthodoxer Schlaf, Tiefschlaf, in } \\
\text { tierexperimentellen Studien SWS } 2\end{array}$ \\
\hline $\begin{array}{l}\text { NREM- } \\
\text { Schlafstadium } 4\end{array}$ & $\geq 50 \%$ hohe $\delta$-Wellen & $\begin{array}{l}\text { Keine Augenbewegungen, EEG-Artefakte, } \\
\text { Abnahme des Muskeltonus }\end{array}$ & $\begin{array}{l}\text { Orthodoxer Schlaf, Tiefschlaf, in } \\
\text { tierexperimentellen Studien SWS } 2\end{array}$ \\
\hline REM-Schlaf & $\theta$-Aktivität, Sägezahnwellen & $\begin{array}{l}\text { Konjugierte rasche Augenbewegungen, } \\
\text { niedriger Muskeltonus mit phasischer } \\
\text { Aktivierung }\end{array}$ & Paradoxer Schlaf, Traumschlaf \\
\hline Movement Time & $\begin{array}{l}>50 \% \text { der Epoche durch } \\
\text { Bewegungsartefakte gestört, } \\
\text { so dass eine Zuordnung zu } \\
\text { einem anderen Stadium } \\
\text { nicht möglich ist }\end{array}$ & & \\
\hline
\end{tabular}

- REM-Dichte: Häufigkeit schneller Augenbewegungen bezogen auf die REM-Schlafphasen.

- REM-Periodendauer: Dauer einer REM-Schlafphase.

- Arousal: Aufweckreaktion, Desynchronisation der EEG.

\subsection{Aktigrafie}

Neben der PSG gibt es als weitere Messmethode zur Erfassung von Schlafgewohnheiten und Schlafstörungen die Aktigrafie. Mittels eines am Handgelenk zu tragendem Aktivitätsmessgeräts wird die Bewegung der Probanden gemessen und über einen Zeitraum von z. B. 14 Tagen gespeichert. Die Aktigrafie kann die Diagnostik von Schlafstörungen ergänzen, wenn z. B. ein Schlaftagebuch geführt wird und die aktigrafischen Ergebnisse hiermit verglichen werden. Das Aktimeter ist in der Lage, ein Schlaf-Wach-Muster zu berechnen, aus denen auch Parameter wie Schlaf-Latenz, Schlafdauer und Schlaf-Effizienz hervorgehen. Diese Messgrößen sind jedoch nicht immer zuverlässig und können eine PSG nicht ersetzen. 


\subsection{Literaturrecherche}

Das Ziel der Literaturrecherche ist, eine systematische Übersichtsarbeit zu erstellen, die sich mit der Wirkung von Nikotin auf den Schlaf, auf das dopaminerge System und auf die Hormone der HHN-Achse beschäftigt. Insbesondere soll hierbei der Nikotinentzug untersucht werden, um mögliche Prädiktoren für einen Rückfall zu identifizieren. Diesbezüglich sind auch Studien einbezogen, die Substanzen im Nikotinentzug getestet haben, falls die gemessenen Parameter auch ohne die Substanzen oder unter Placebobedingungen bestimmt wurden. Neben Studien mit menschlichen Probanden sind auch tierexperimentelle Arbeiten (in vivo) von Interesse. Ein weiteres Ziel der Literaturrecherche ist, Studien zu finden, die Nichtraucher nach einer Nikotingabe mit Gelegenheitsrauchern oder Rauchern oder die die genannten Probandengruppen im Entzug vergleichen.

Die Literaturrecherche ist in mehrere Abschnitte unterteilt.

1. Zuerst sollen Studien gefunden werden, in denen Tieren Nikotin verabreicht wurde, und in denen die Konsequenzen für

- objektive Schlafparameter und

- die Hormone der HHN-Achse

untersucht wurden.

2. Anschließend werden Studien gesucht, in denen Nichtrauchern Nikotin verabreicht wurde, und in denen die Folgen für

- objektive Schlafparameter,

- das dopaminerge System und

- die Hormone der HHN-Achse

bestimmt wurden. 
3. Es schließt sich die Literaturrecherche nach Veröffentlichungen an, die sich mit einem Nikotinentzug bei Gelegenheitsrauchern beschäftigten. Auch hier sollen die Konsequenzen für

- objektive Schlafparameter,

- das dopaminerge System und

- die Hormone der HHN-Achse

dargestellt sein.

4. Zum Schluss sollen Studien, die sich mit einem Nikotinentzug bei Rauchern auseinandersetzt haben, in die Übersichtsarbeit aufgenommen werden. Hier sind ebenfalls die Folgen des Entzugs für

- objektive Schlafparameter,

- das dopaminerge System und

- die Hormone der HHN-Achse

von Interesse.

Für diese Suche werden ausgewählte Stichwörter (siehe 2.5.1 bis 2.5.3) in die elektronischen Datenbank PubMed eingegeben. Einbezogen sind alle Studien, die bis zum 15. August 2012 publiziert wurden und in PubMed aufgeführt sind.

Die Stichwörter werden miteinander kombiniert und die Trefferlisten nach Originalstudien durchsucht, die dem einzelnen Suchziel entsprechen. Hierbei sind alle Studienarten von Interesse. Veröffentlichungen, die die Anforderungen in befriedigendem Maße erfüllen, sind in die vorliegende Übersichtsarbeit aufgenommen worden. Zusätzlich wird in den Quellenangaben jeder Studie nach weiteren Veröffentlichungen gesucht, die ebenfalls die Suchkriterien erfüllen.

Die Studien sind in Kapitel 3 und im Anhang in Tabellen aufgeführt. Hierbei konzentriert sich die Darstellung auf die für diese Übersichtsarbeit relevanten Informationen. Zudem sind nur signifikante Ergebnisse dargestellt (falls nicht anderes vermerkt) und diese in Bezug zu den Baseline-Messungen angegeben. Wenn keine Veränderungen gemessen wurden, sind diese zumeist 
nicht aufgeführt. Ebenso wurde auf die Angabe der Kontrollgruppen und Baseline-Messungen verzichtet.

\subsubsection{Objektive Schlafparameter}

Die verwendeten Stichwörter, Limitierungen und Kriterien bei der Suche nach Studien, die sich mit dem Schlaf auseinandergesetzt haben, lauten im Einzelnen:

\section{Tiere}

1. Stichwörter und Limitierungen

- nicotine, smoking, tobacco, cigarettes, cigarette smoke.

- sleep, sleeping, polysomnography, PSG, rapid-eye-movement, REM, NREM,

NonREM, Non-REM, actigraphy, actimetry, actimeter, actometer, actiwatch.

- rats, mice, cats oder die Limitierung, dass nur nach tierexperimentellen Untersuchungen gesucht wird.

2. Kriterien

- Tieren wurde Nikotin verabreicht.

- Es wurden objektive Schlafparameter erfasst.

- Ein Vergleich dieser Parameter vor und nach Nikotingabe ist möglich.

\section{Nichtraucher}

1. Stichwörter

- nicotine, smoking, tobacco, cigarettes.

- sleep, sleeping, polysomnography, PSG, rapid-eye-movement, REM, NREM,

NonREM, Non-REM, actigraphy, actimetry, actimeter, actometer, actiwatch. 
- nonsmoker(s), non-smoker(s), nonsmoking, non-smoking.

2. Kriterien

- Nichtrauchern wurde Nikotin verabreicht.

- Es wurden objektive Schlafparameter erfasst.

- Ein Vergleich dieser Parameter vor und nach Nikotingabe ist möglich.

\section{Gelegenheitsraucher}

1. Stichwörter

- nicotine, smoking, tobacco, cigarettes.

- sleep, sleeping, polysomnography, PSG, rapid-eye-movement, REM, NREM, NonREM, Non-REM, actigraphy, actimetry, actimeter, actometer, actiwatch.

- occasional smoker(s), smoking occasionally.

- withdrawal, cessation, deprivation, detoxification, abstinence.

2. Kriterien

- Gelegenheitsraucher haben einen Nikotinentzug durchgeführt.

- Es wurden objektive Schlafparameter erfasst.

- Ein Vergleich dieser Parameter vor und im Entzug ist möglich.

\section{Raucher}

1. Stichwörter

- nicotine, smoking, tobacco, cigarettes.

- sleep, sleeping, polysomnography, PSG, rapid-eye-movement, REM, NREM, NonREM, Non-REM, actigraphy, actimetry, actimeter, actometer, actiwatch. 
- withdrawal, cessation, deprivation, detoxification, abstinence.

2. Kriterien

- Raucher haben einen Nikotinentzug durchgeführt.

- Es wurden objektive Schlafparameter erfasst.

- Ein Vergleich dieser Parameter vor und im Entzug ist möglich.

- Publikationen, in denen eine Nikotinersatztherapie durchgeführt wurde oder andere Substanzen im Nikotinentzug getestet wurden, werden nur berücksichtigt, wenn die objektiven Schlafparameter auch ohne diese Verfahren oder unter Placebobedingungen bestimmt wurden.

\subsubsection{Dopaminerges System}

Die verwendeten Stichwörter und Kriterien bei der Suche nach Studien, die sich mit Parametern des dopaminergen Systems beschäftigt haben, lauten im Einzelnen:

\section{Nichtraucher}

1. Stichwörter

- nicotine, smoking, tobacco, cigarettes.

- nonsmoker(s), non-smoker(s), nonsmoking, non-smoking.

- dopamine.

2. Kriterien

- Nichtrauchern wurde Nikotin verabreicht.

- Messgrößen des dopaminergen Systems wurden bestimmt.

- Ein Vergleich dieser Messgrößen vor und nach Nikotingabe ist möglich. 


\section{Gelegenheitsraucher}

1. Stichwörter

- nicotine, smoking, tobacco, cigarettes.

- occasional smoker(s), smoking occasionally.

- withdrawal, cessation, deprivation, detoxification, abstinence.

- dopamine.

2. Kriterien

- Gelegenheitsraucher haben einen Nikotinentzug durchgeführt.

- Messgrößen des dopaminergen Systems wurden bestimmt.

- Ein Vergleich dieser Messgrößen vor und im Entzug ist möglich.

\section{Raucher}

1. Stichwörter

- nicotine, smoking, tobacco, cigarettes.

- withdrawal, cessation, deprivation, detoxification, abstinence.

- dopamine.

2. Kriterien

- Raucher haben einen Nikotinentzug durchgeführt.

- Messgrößen des dopaminergen Systems wurden bestimmt.

- Ein Vergleich dieser Messgrößen vor und im Entzug ist möglich. 
- Publikationen, in denen eine Nikotinersatztherapie durchgeführt wurde oder andere Substanzen im Nikotinentzug getestet wurden, werden nur berücksichtigt, wenn die Parameter des dopaminergen Systems auch ohne diese Verfahren oder unter Placebobedingungen bestimmt wurden.

\subsubsection{Hypothalamus-Hypophysen-Nebennieren-Achse}

Die verwendeten Stichwörter, Limitierungen und Kriterien bei der Suche nach Studien, die sich mit der HHN-Achse auseinandergesetzt haben, lauten im Einzelnen:

\section{Tiere}

1. Stichwörter und Limitierungen

- nicotine, smoking, tobacco, cigarettes, cigarette smoke.

- cortisol, hypothalamic-pituitary-adrenal axis, hypothalamic-hypophyseal-adrenal axis, HPA axis, glucocorticoid(s), corticosteroid(s).

- rats, mice, cats oder die Limitierung, dass nur nach tierexperimentellen Untersuchungen gesucht wird.

2. Kriterien

- Tieren wurde Nikotin verabreicht.

- Hormone der HHN-Achse wurden bestimmt.

- Ein Vergleich der Hormonwerte vor und nach Nikotingabe ist möglich.

\section{Nichtraucher}

1. Stichwörter

- nicotine, smoking, tobacco, cigarettes. 
- cortisol, hypothalamic-pituitary-adrenal axis, hypothalamic-hypophyseal-adrenal axis, HPA axis, glucocorticoid(s), corticosteroid(s).

- nonsmoker(s), non-smoker(s), nonsmoking, non-smoking.

2. Kriterien

- Nichtrauchern wurde Nikotin verabreicht.

- Hormone der HHN-Achse wurden bestimmt.

- Ein Vergleich der Hormonwerte vor und nach Nikotingabe ist möglich.

\section{Gelegenheitsraucher}

1. Stichwörter

- nicotine, smoking, tobacco, cigarettes.

- cortisol, hypothalamic-pituitary-adrenal axis, hypothalamic-hypophyseal-adrenal axis, HPA axis, glucocorticoid(s), corticosteroid(s).

- occasional smoker(s), smoking occasionally.

- withdrawal, cessation, deprivation, detoxification, abstinence.

2. Kriterien

- Gelegenheitsraucher haben einen Nikotinentzug durchgeführt.

- Hormone der HHN-Achse wurden bestimmt.

- Ein Vergleich der Hormonwerte vor und nach Nikotingabe ist möglich. 


\section{Raucher}

1. Stichwörter

- nicotine, smoking, tobacco, cigarettes.

- cortisol, hypothalamic-pituitary-adrenal axis, hypothalamic-hypophyseal-adrenal axis, HPA axis, glucocorticoid(s), corticosteroid(s).

- withdrawal, cessation, deprivation, detoxification, abstinence.

\section{Kriterien}

- Raucher haben einen Nikotinentzug durchgeführt.

- Hormone der HHN-Achse wurden bestimmt.

- Ein Vergleich der Hormonwerte vor und nach Nikotingabe ist möglich.

- Publikationen, in denen eine Nikotinersatztherapie durchgeführt wurde oder andere Substanzen im Nikotinentzug getestet wurden, werden nur berücksichtigt, wenn die Hormone der HHN-Achse auch ohne diese Verfahren oder unter Placebobedingungen bestimmt wurden. 


\section{Ergebnisse}

\section{1 Übersicht}

Durch die Literaturrecherche wurden 3037 Veröffentlichungen gefunden, von denen sich ein Großteil mit der HHN-Achse auseinandergesetzt hat. Aus diesen Ergebnissen entsprechen 93 Studien den Suchkriterien und diese wurden in den Ergebnisteil aufgenommen. Eine Übersicht hierzu bietet Tabelle 3.1 .

Tabelle 3.1: Anzahl an Suchergebnissen und aufgenommenen Studien.

\begin{tabular}{lrr}
\hline Thema & $\begin{array}{r}\text { Suchergebnisse } \\
\text { insgesamt }\end{array}$ & Aufgenommene Studien \\
\hline Schlaf & 816 & 25 \\
Dopaminerges System & 847 & 5 \\
HHN-Achse & 1374 & 63 \\
Alle & 3037 & 93 \\
\hline
\end{tabular}

Wieviele Studien auf die einzelnen Suchabschnitte entfallen, wird in Tabelle 3.2 dargestellt, die auch die Anzahl der beteiligten Forschungsgruppen enthält.

Ein Großteil der Studien sind nicht kontrollierte Studien. Die Verteilung der Studiendesigns ist in Tabelle 3.3 ersichtlich.

Im Folgenden werden die konkreten Studienergebnisse dargestellt. Angegeben sind jeweils nur signifikante Änderungen, falls es im Text nicht anders vermerkt ist. Die gefundenen Veröffentlichungen sind neben den im Ergebnisteil aufgeführten Tabellen im Anhang in einer ausführlichen Übersicht dargestellt (siehe Tabellen 6.1-6.7). 
Tabelle 3.2: Anzahl an Studien und Forschungsgruppen.

\begin{tabular}{lrrr}
\hline Thema & \multicolumn{2}{c}{ Anzahl der Studien | Anzahl der Forschungsgruppen } \\
\cline { 2 - 4 } & Schlaf & Dopaminerges System & HHN-Achse \\
\hline Tierexperimentelle Untersuchungen & $8 \mid 6$ & nicht untersucht & $37 \mid 26$ \\
Nikotingabe an Nichtraucher & $7 \mid 3$ & $0 \mid 0$ & $6 \mid 4$ \\
Gelegenheitsraucher im Entzug & $0 \mid 0$ & $0 \mid 0$ & $0 \mid 0$ \\
Raucher im Entzug & $10 \mid 6$ & $5 \mid 4$ & $20 \mid 12$ \\
\hline
\end{tabular}

Tabelle 3.3: Studiendesigns der Studien.

\begin{tabular}{|c|c|c|c|c|c|}
\hline \multirow[t]{2}{*}{ Studiendesign } & \multicolumn{2}{|c|}{ Nichtraucher } & \multicolumn{3}{|c|}{ Raucher } \\
\hline & Schlaf & HHN-Achse & Schlaf & HHN-Achse & Dopaminerges System \\
\hline Nicht kontrolliert & 2 & 4 & 6 & 12 & 5 \\
\hline Kontrolliert & 1 & & 1 & 6 & \\
\hline Einfachblind, kontrolliert & & 2 & & & \\
\hline $\begin{array}{l}\text { Doppelblind, randomisiert, } \\
\text { placebokontrolliert }\end{array}$ & & & 3 & & \\
\hline $\begin{array}{l}\text { Doppelblind, randomisiert, } \\
\text { placebokontrolliert, Cross-over }\end{array}$ & 4 & & & 2 & \\
\hline
\end{tabular}




\subsection{Objektive Schlafparameter}

\subsubsection{Nikotingabe in tierexperimentellen Untersuchungen}

Die Suche erbrachte 224 Suchergebnisse, von denen acht Studien den Suchkriterien entsprechen und in den Ergebnisteil aufgenommen worden sind. Sechs Forschungsgruppen haben sich in einem Zeitraum von 1965 bis 2010 mit den Auswirkungen einer Nikotingabe auf den Schlaf von Tieren beschäftigt. Die Arbeitsgruppe um R. Salín-Pascual und R. Drucker-Colín trägt drei Veröffentlichungen zu diesem Thema bei. Alle Studien untersuchten die akute Reaktion auf eine Nikotingabe, zwei Veröffentlichungen beobachteten zusätzlich einen längerer Zeitraum von bis zu 14 Tagen. In drei Studien wurde zudem der Absetztag nach einer Nikotingabe untersucht, die Ergebnisse hierzu finden sich im Abschnitt „Nikotinentzug“.

\section{Akute Nikotingabe}

Tabelle 3.4 fasst die ersten vier Studien zusammen, in denen die Konsequenzen einer Nikotingabe auf den Schlaf von Katzen untersucht wurden.

Domino und Yamamoto (1965) injizierten schlafenden Katzen intravenös (i. v.) Nikotin (0,005$0,01 \mathrm{mg} / \mathrm{kg}$ ) und konnten wenige Minuten später eine Desynchronisation der EEG-Aktivität und eine kurze Aufwachreaktion feststellen. Darauf verfielen die Tiere in einen tieferen Schlaf als vor der Nikotingabe. Nach circa 15-25 Minuten war der REM-Schlafanteil gegenüber den Messungen vor der Nikotingabe erhöht. Jewett und Norton (1966) beobachteten eine von der Nikotindosis abhängige Veränderung der polysomnografischen Parameter. Sie verabreichten Katzen Nikotin subkutan (s.c.) und konnten für die Dosis von $0,05 \mathrm{mg} / \mathrm{kg}$ Nikotin folgendes feststellen: Die Zeit, die die Tiere im REM-Schlaf und im SWS 2 verbrachten, stieg zusammen mit der Gesamtschlafzeit an. Nach einer Dosis von $0,1 \mathrm{mg} / \mathrm{kg}$ Nikotin zeigte sich ein Anstieg der REM-Schlafzeit. Nach beiden Dosen konnte eine geringere Wachzeit der Tiere beobachtet werden. Für die in dieser Studie verwendete Höchstdosis $(0,2 \mathrm{mg} / \mathrm{kg}$ Nikotin) wurden gegenteilige Veränderungen beobachtet: Die Zeit im REM-Schlaf und SWS 2 nahm zusammen mit der Gesamtschlaftzeit ab. Hinzu kam bei dieser Dosis eine Zunahme der Wachzeit. Die Elektromyografie (EMG) wurde durch alle Nikotindosen beeinflusst und zeigte eine erhöhte Muskelaktivität. In der Veröffentlichung von 
Tabelle 3.4: Auswirkungen einer akuten Nikotingabe auf polysomnografische Parameter bei Katzen. ${ }^{a}$

\begin{tabular}{|c|c|c|c|c|}
\hline \multirow[t]{2}{*}{ Autoren } & \multirow[t]{2}{*}{ Nikotindosis } & \multicolumn{3}{|c|}{ Nach Nikotingabe } \\
\hline & & REM-Parameter & sws & WZ, TST \\
\hline $\begin{array}{l}\text { Domino, } \\
\text { Yamamoto } \\
1965\end{array}$ & $\begin{array}{l}0,005- \\
0,01 \mathrm{mg} / \mathrm{kg} \\
\text { Nikotin i. v. }\end{array}$ & $\begin{array}{l}\text { REM-Schlaf } \uparrow \text { (nach } \\
15-25 \text { min) }\end{array}$ & $\begin{array}{l}\text { SWS } 2 \uparrow(\text { nach } \\
\text { 5-15 min) }\end{array}$ & \\
\hline $\begin{array}{l}\text { Jewett, } \\
\text { Norton } \\
1966\end{array}$ & $\begin{array}{l}0,05,0,1 \\
0,2 \mathrm{mg} / \mathrm{kg} \\
\text { Nikotin s. c. }\end{array}$ & $\begin{array}{l}\text { REM-Schlaf } \uparrow(0,05 \text { und } \\
0,1 \mathrm{mg} / \mathrm{kg}), \downarrow(0,2 \mathrm{mg} / \mathrm{kg})\end{array}$ & $\begin{array}{l}\text { SWS } 1 \uparrow(0,2 \mathrm{mg} / \mathrm{kg}) ; \\
\mathrm{SWS} 2 \uparrow(0,05 \mathrm{mg} / \mathrm{kg}), \\
\downarrow(0,2 \mathrm{mg} / \mathrm{kg})\end{array}$ & $\begin{array}{l}\text { WZ } \downarrow(0,05 \text { und } \\
0,1 \mathrm{mg} / \mathrm{kg}), \uparrow(0,2 \mathrm{mg} / \mathrm{kg}) ; \\
\mathrm{TST} \uparrow(0,05 \mathrm{mg} / \mathrm{kg}), \downarrow \\
(0,2 \mathrm{mg} / \mathrm{kg})\end{array}$ \\
\hline $\begin{array}{l}\text { Velazquez- } \\
\text { Moctezuma } \\
\text { et al. } 1990\end{array}$ & $\begin{array}{l}0,0092 \mathrm{mg} \\
\text { Nikotin in die } \\
\text { Formatio } \\
\text { reticularis }\end{array}$ & REM-Schlaf $\uparrow ;$ REM-Latenz $\downarrow$ & SWS $1 \downarrow$ & $\mathrm{WZ} \downarrow$ \\
\hline $\begin{array}{l}\text { Vazquez et } \\
\text { al. } 1996\end{array}$ & $\begin{array}{l}17,5,35 \\
52,5 \mathrm{mg} \mathrm{NP}\end{array}$ & $\begin{array}{l}\text { REM-Schlaf } \downarrow \text { (35 und } \\
52,5 \mathrm{mg}) ; \text { REM-Periodendauer } \\
\uparrow(17,5 \mathrm{mg}) ; \text { REM-Latenz } \downarrow \\
(17,5 \mathrm{mg}) ; \text { REM-Frequenz } \downarrow \\
\text { (alle Dosen) }\end{array}$ & & WZ $\uparrow$ (alle Dosen) \\
\hline
\end{tabular}

$\overline{\left.{ }^{a} \text { Kurzbezeichnungen: Beta2-Knockout ( } \beta 2-K O\right), ~ T a g(e) ~(d), ~ S t u n d e n ~(h), ~ i n t r a p e r i t o n e a l ~(i . ~ p .), ~ i n t r a v e n o ̈ s ~(i . ~ v .), ~ M i l-~}$ ligramm pro Kilogramm (mg/kg), Nikotinpflaster (NP), Non-Rapid-Eye-Movement (NREM), Polysomnografie (PSG), Rapid-Eye-Movement (REM), subkutan (s. c.), Slow-Wave-Sleep (SWS), Total-Sleep-Time (TST), Wachzeit (WZ), signifikant erhöht $(\uparrow)$, gleichbleibend $(\leftrightarrow)$, signifikant erniedrigt $(\downarrow)$.

Velazquez-Moctezuma et al. (1990) wurde Katzen 0,0092 mg Nikotin in die Formatio reticularis des Hirnstamms injiziert und über die Dauer von drei Stunden EEG durchgeführt. Es zeigte sich ein Anstieg der REM-Schlafzeit, ein Abfall der REM-Latenz, des prozentualen Anteils des SWS 1 sowie der Wachzeit. Der prozentuale Anteil des SWS 2 änderte sich nicht. Wie in der Studie von Jewett und Norton (1966) beobachteten Vazquez et al. (1996) dosisabhängige Änderungen der polysomnografischen Parameter. Nach dem Einsatz von Nikotinpflastern der geringsten Dosierung (17,5 mg Nikotin) über zehn Stunden wurden eine geringere REM-Latenz und eine erhöhte REMPeriodendauer gemessen. Die Gesamtzeit im REM-Schlaf war nicht verändert. Wurde die doppelte bzw. dreifache Dosis verwendet, fiel die REM-Schlafzeit ab. Nach allen Dosen kam es zu einem Abfall der REM-Frequenz und zu einer Zunahme der Wachzeit. Der SWS wurde durch Nikotin nicht beeinflusst.

In Tabelle 3.5 sind vier Studien zu finden, die sich mit einer akuten Nikotingabe an Ratten und Mäusen auseinandergesetzt haben. 
Tabelle 3.5: Auswirkungen einer akuten Nikotingabe auf polysomnografische Parameter bei Nagern.

\begin{tabular}{|c|c|c|c|c|}
\hline \multirow[t]{2}{*}{ Autoren } & \multirow[t]{2}{*}{ Nikotindosis } & \multicolumn{3}{|c|}{ Nach Nikotingabe } \\
\hline & & REM-Parameter & SWS & WZ, TST \\
\hline $\begin{array}{l}\text { Salín- } \\
\text { Pascual et } \\
\text { al. } 1999\end{array}$ & $\begin{array}{l}0,1,0,25,0,5 \\
1 \mathrm{mg} / \mathrm{kg} \\
\text { Nikotin s. c. }\end{array}$ & $\begin{array}{l}\text { REM-Schlaf } \downarrow \text { ( } 0,5 \text { und } 1 \mathrm{mg} / \mathrm{kg}) \text {; } \\
\text { REM-Latenz } \uparrow(0,5 \text { und } \\
1 \mathrm{mg} / \mathrm{kg}) ; \text { REM-Frequenz } \downarrow \text { (alle } \\
\text { Dosen) }\end{array}$ & $\begin{array}{l}\text { SWS } 2 \downarrow(0,5 \text { und } \\
1 \mathrm{mg} / \mathrm{kg})\end{array}$ & $\begin{array}{l}\text { WZ } \uparrow(0,5 \text { und } \\
1 \mathrm{mg} / \mathrm{kg})\end{array}$ \\
\hline $\begin{array}{l}\text { Guzmán- } \\
\text { Marín et al. } \\
2001\end{array}$ & $\begin{array}{l}0,1 \mathrm{mg} / \mathrm{kg} \\
\text { Nikotin s. c. }\end{array}$ & REM-Periodendauer $\uparrow$ & & \\
\hline \multirow[t]{2}{*}{$\begin{array}{l}\text { Léna et al. } \\
2004\end{array}$} & $\begin{array}{l}\text { WT: } 0,5,1, \\
2 \mathrm{mg} / \mathrm{kg} \\
\text { Nikotin i. p. }\end{array}$ & $\begin{array}{l}\text { REM-Schlaf } \downarrow \text { ( } 1 \text { und } 2 \mathrm{mg} / \mathrm{kg}, 1 \text {. } \\
\text { Stunde) }\end{array}$ & $\begin{array}{l}\text { SWS } \downarrow(1 \text { und } 2 \mathrm{mg} / \mathrm{kg} \text {, } \\
1 . \text { Stunde }),(1 \text { und } \\
2 \mathrm{mg} / \mathrm{kg}, 2 . \text { Stunde })\end{array}$ & $\begin{array}{l}\text { WZ } \uparrow(1 \text { und } 2 \mathrm{mg} / \mathrm{kg} \text {, } \\
1 . \text { Stunde }), \downarrow \text { ( } 1 \text { und } \\
2 \mathrm{mg} / \mathrm{kg}, 2 . \text { Stunde })\end{array}$ \\
\hline & $\begin{array}{l}\beta 2-\mathrm{KO}: 0,5,1 \\
2 \mathrm{mg} / \mathrm{kg} \\
\text { Nikotin i.p. }\end{array}$ & & & $\begin{array}{l}\mathrm{WZ} \downarrow(2 \mathrm{mg} / \mathrm{kg}, 3 . \\
\text { Stunde) }\end{array}$ \\
\hline $\begin{array}{l}\text { Vázquez- } \\
\text { Palacios et } \\
\text { al. } 2010\end{array}$ & $\begin{array}{l}0,4 \mathrm{mg} / \mathrm{kg} \\
\text { Nikotin s.c. }\end{array}$ & REM-Schlaf $\downarrow$; REM-Latenz $\uparrow$ & SWS $2 \downarrow$ & $\mathrm{WZ} \uparrow$ \\
\hline
\end{tabular}

Salín-Pascual et al. (1999) untersuchten die Konsequenzen einer Nikotingabe auf den Schlaf von Ratten. Sie spritzen den Tieren Nikotin in steigender Dosierung s. c. Auch in dieser Studie wurden dosisabhängige Veränderungen gefunden: Erst mit höherer Dosierung kam es zu einem Abfall der REM-Schlafzeit und der Zeit im SWS 2, sowie zu einer Erhöhung der REM-Latenz und der Wachzeit. Nach allen Nikotindosen verringerte sich die REM-Frequenz. Guzmán-Marín et al. (2001) konnten bei einer Nikotindosis von $0,1 \mathrm{mg} / \mathrm{kg}$ s. c. eine Zunahme der REM-Periodendauer beobachten. In der Studie von Léna et al. (2004) wurden zwei unterschiedliche Mäusepopulationen untersucht - zum einen Wildtyp-Mäuse, zum anderen Mäuse, denen die $\beta 2$-Untereinheit des nACh-Rezeptors fehlte. Nach intraperitonealer (i.p.) Injektion von 1 oder $2 \mathrm{mg} / \mathrm{kg}$ Nikotin zeigte sich bei den WildtypMäusen in der ersten Ableitungsstunde ein Abfall der REM- und NREM-Schlafzeit und somit eine Zunahme der Wachzeit. In der zweiten Stunde nach Nikotingabe konnte ein umgekehrtes Bild beobachtet werden, hier kam es zu einer Zunahme der NREM-Schlafzeit und zu einer Abnahme der Wachzeit. Danach konnten keine weiteren Veränderungen polysomnografischer Parameter festgestellt werden. Diese Veränderungen zeigten die genmanipulierten Mäusen nicht. Bei ihnen kam es lediglich in der dritten Stunde nach $2 \mathrm{mg} / \mathrm{kg}$ Nikotin i.p. zu einer Abnahme der Wachzeit. 
Vázquez-Palacios et al. (2010) konnten nach einmaliger Gabe von 0,4 mg/kg Nikotin einen Anstieg der Wachzeit und damit einhergehend eine Abnahme der REM-Schlafzeit und der Zeit im SWS 2 messen. Die REM-Latenz stieg nach der Nikotingabe an.

Zusammenfassend lässt sich in vielen Studien und beim direkten Vergleich der Studienergebnisse eine dosisabhängige Veränderung der polysomnografischen Parameter nach einer akuten Nikotingabe nachweisen. Die Wachzeit stieg nach höheren Nikotindosen an, verbunden mit einer Abnahme der REM-Schlafzeit und der Zeit im SWS. Betrachtet man jedoch die Auswirkungen geringerer Dosen, wurden in vielen Arbeiten ein Anstieg der REM-Schlafzeit und eine unveränderte Zeit im SWS gefunden.

\section{Chronische Nikotingabe}

Die beiden Studien, in denen Ratten über mehrere Tage Nikotin verabreicht wurde, sind in Tabelle $3.6 \mathrm{zu}$ finden.

Salín-Pascual et al. (1999) beobachteten ab dem dritten Applikationstag für eine Dosis von 0,1 mg/kg Nikotin s. c. einen Anstieg der REM-Schlafzeit. Für 0,5 mg/kg Nikotin wurde ab dem ersten Tag ein geringere REM-Schlafzeit gemessen. Die Zeit im SWS verkürzte sich nach Gabe von beiden Nikotindosen. Auch nach 7- bzw. 14-tägiger Nikotingabe konnten Vázquez-Palacios et al. (2010) dieselben Ergebnisse wie nach einer akuten Gabe messen (Anstieg der Wachzeit mit einer Abnahme der REM-Schlafzeit und der Zeit im SWS 2).

Zusammengefasst finden sich auch an dieser Stelle dosisabhängige Veränderungen der REM-

Tabelle 3.6: Auswirkungen einer chronischen Nikotingabe auf polysomnografische Parameter bei Ratten.

\begin{tabular}{lllll}
\hline \multirow{2}{*}{ Autoren } & Nikotindosis & \multicolumn{3}{c}{ Nach Nikotingabe } \\
\cline { 3 - 5 } & & REM-Parameter & SWS & WZ, TST \\
\hline Salín-Pascual et & $4 \mathrm{~d} 0,5,1 \mathrm{mg} / \mathrm{kg}$ & REM-Schlaf $\uparrow(0,1 \mathrm{mg} / \mathrm{kg}$, ab & SWS $2 \downarrow$ (alle & WZ $\downarrow(0,1 \mathrm{mg} / \mathrm{kg}), \uparrow$ \\
al. 1999 & Nikotin s. c. & Tag 3), $\downarrow(0,5 \mathrm{mg} / \mathrm{kg})$ & Dosen $)$ & $(0,5 \mathrm{mg} / \mathrm{kg})$ \\
$\begin{array}{l}\text { Vázquez-Palacios } \\
\text { et al. 2010 }\end{array}$ & 7 und 14 d 0,4 mg $/ \mathrm{kg}$ & REM-Schlaf $\downarrow ;$ REM-Latenz $\uparrow$ & SWS $2 \downarrow$ & WZ $\uparrow$ \\
\hline
\end{tabular}


Schlafzeit. Geringe chronische Nikotindosen verursachten einen Anstieg, höhere Dosen einen Abfall der REM-Schlafzeit. Die Zeit im SWS wurde durch eine mehrtägige Nikotingabe verringert.

\section{Nikotinentzug}

Tabelle 3.7 beinhaltet drei Studien, die auch die Zeit nach der Nikotingabe untersucht haben.

Vazquez et al. (1996) konnten am Absetztag nach der Nikotingabe mit einer Ausnahme keine Unterschiede in den polysomnografischen Parametern in Bezug zur Baseline-Messung ausmachen. War die Gesamtzeit, die die Tiere unter 35 und 52,5 mg Nikotinpflastern im REM-Schlaf verbrachten, noch erniedrigt, konnte am Absetztag keine solche Veränderung beobachtet werden. Lediglich die REM-Periodendauer stieg - nach Meinung der Autoren verbunden mit einer geringeren REM-Frequenz - an. Der Anstieg des REM-Schlafs unter 0,1 mg/kg Nikotin ab dem dritten Applikationstag konnte von Salín-Pascual et al. (1999) auch am Absetztag nach der Nikotingabe beobachtet werden. Der unter mehrtägiger Gabe von $0,5 \mathrm{mg} / \mathrm{kg}$ Nikotin gemessene Abfall der REM-Schlafzeit zeigte sich hingegen an dem folgenden Tag ohne Nikotin nicht. Stattdessen kam es zu einem Anstieg der REM-Schlafzeit. Vázquez-Palacios et al. (2010) stellten auch sieben Tage nach einer 14-tägigen Nikotingabe fest, dass die REM-Schlafzeit und die Zeit im SWS 2 vermindert und die Wachzeit und die REM-Latenz erhöht waren.

Zusammenfassend ist ein gemeinsamer Trend aufgrund der unterschiedlichen Methoden und Resultate der Studien nicht erkennbar.

Tabelle 3.7: Auswirkungen eines Nikotinentzugs auf polysomnografische Parameter bei Katzen und Ratten.

\begin{tabular}{|c|c|c|c|c|c|c|}
\hline \multirow[t]{2}{*}{ Autoren } & \multirow[t]{2}{*}{ Versuchstiere } & \multirow[t]{2}{*}{ Nikotindosis } & \multirow[t]{2}{*}{ Entzugszeit } & \multicolumn{3}{|c|}{ Im Entzug } \\
\hline & & & & REM-Parameter & SWS & $\begin{array}{l}\text { WZ, } \\
\text { TST }\end{array}$ \\
\hline $\begin{array}{l}\text { Vazquez et al. } \\
1996\end{array}$ & Katzen & $17,5,35,52,5 \mathrm{mg} \mathrm{NP}$ & $24 \mathrm{~h}$ & $\begin{array}{l}\text { REM-Periodendauer } \\
\uparrow(35 \text { und } 52,5 \mathrm{mg})\end{array}$ & & \\
\hline $\begin{array}{l}\text { Salín-Pascual et } \\
\text { al. } 1999\end{array}$ & Ratten & $\begin{array}{l}4 \mathrm{~d} 0,5,1 \mathrm{mg} / \mathrm{kg} \\
\text { Nikotin s.c. }\end{array}$ & $24 \mathrm{~h}$ & $\begin{array}{l}\text { REM-Schlaf } \uparrow \text { (alle } \\
\text { Dosen) }\end{array}$ & & \\
\hline $\begin{array}{l}\text { Vázquez-Palacios } \\
\text { et al. } 2010\end{array}$ & Ratten & $\begin{array}{l}7 \text { und } 14 \mathrm{~d} 0,4 \mathrm{mg} / \mathrm{kg} \\
\text { Nikotin s. c. }\end{array}$ & $7 \mathrm{~d}$ & $\begin{array}{l}\text { REM-Schlaf } \downarrow \text {; } \\
\text { REM-Latenz } \uparrow\end{array}$ & SWS $2 \downarrow$ & $\mathrm{WZ} \uparrow$ \\
\hline
\end{tabular}




\section{Zusammenfassung}

Tabelle 3.8 fasst die Ergebnisse der Studien zusammen, die eine Nikotingabe in tierexperimentellen Untersuchungen beobachtet haben.

\subsubsection{Nikotingabe an Nichtraucher}

Die Suche erbrachte 191 Suchergebnisse, von denen sieben in den Ergebnisteil aufgenommen worden sind. Drei Forschungsgruppen haben sich in der Vergangenheit zum Ziel gesetzt, die Wirkung von Nikotin auf den Schlaf von Nichtrauchern zu erforschen. Fünf Veröffentlichungen stammen aus der Forschungsgruppe um R. Salín-Pascual und R. Drucker-Colín. Vier der sieben Studien sind in den neunziger Jahren des vergangenen Jahrhunderts erschienen, die aktuellste Studie ist von 2009.

Mehr als die Hälfte der Arbeiten beschäftigte sich mit depressiven Probanden und ging der Frage nach, ob eine Nikotingabe neben Veränderungen von Schlafparametern auch zu einer Besserung der Depressionssymptomatik führt. Im Folgenden werden zuerst die Studien, die sich mit gesunden Nichtrauchern beschäftigten, erläutert und im Anschluss daran die Studien mit depressiven Probanden dargestellt. Den Abschluss macht eine Studie, die als einzige mittels Aktigrafie die Auswirkungen einer Nikotingabe auf die Aktivität im Schlaf ermittelte.

\section{Gesunde Nichtraucher}

Zwei Forschungsgruppen haben sich mit den Auswirkungen einer Nikotingabe auf den Schlaf von gesunden Nichtrauchern beschäftigt. In Tabelle 3.9 sind die vier Studien aufgeführt.

Davila et al. (1994) verabreichten Nichtrauchern um 18 Uhr 11 mg-Nikotinpflaster und konnten in den darauf folgenden PSG eine Reduktion des prozentualen Anteils des REM-Schlafs, der SchlafEffizienz und der Gesamtschlafzeit beobachten. Die Parameter des NREM-Schlafs waren nicht verändert, die Schlaf-Latenz hingegen verlängerte sich. Gillin et al. (1994) setzten Nikotinpflaster unterschiedlicher Dosierung (7 und $14 \mathrm{mg}$ Nikotin) ein, die zwei Stunden vor Beginn der PSG 
Tabelle 3.8: Auswirkungen einer Nikotingabe auf polysomnografische Parameter in tierexperimentellen Untersuchungen (Zusammenfassung).

\begin{tabular}{|c|c|c|c|c|c|}
\hline & \multirow[t]{2}{*}{ Studienanzahl } & \multirow[t]{2}{*}{ Einheitlichkeit } & \multicolumn{3}{|c|}{ Ergebnisse } \\
\hline & & & REM-Schlaf & SWS 2 & TST \\
\hline Akute Nikotingabe & 8 & & & & \\
\hline geringere Dosis & & überwiegend & $\uparrow$ & $\leftrightarrow$ & $\uparrow \leftrightarrow$ \\
\hline höhere Dosis & & ja & $\downarrow$ & $\downarrow$ & $\downarrow$ \\
\hline Chronische Nikotingabe & 2 & & & & \\
\hline geringere Dosis & & & $\uparrow$ & $\downarrow$ & $\uparrow$ \\
\hline höhere Dosis & & ja & $\downarrow$ & $\downarrow$ & $\downarrow$ \\
\hline Entzug & 3 & nein & $\uparrow \leftrightarrow \downarrow$ & $\leftrightarrow \downarrow$ & $\leftrightarrow \downarrow$ \\
\hline
\end{tabular}

${ }^{a}$ Mindestens $70 \%$ der Studien haben ähnliche Ergebnisse.

Tabelle 3.9: Auswirkung einer akuten Nikotingabe auf polysomnografische Parameter bei gesunden Nichtrauchern.

\begin{tabular}{|c|c|c|c|c|c|c|c|}
\hline \multirow[t]{2}{*}{ Autoren } & \multirow{2}{*}{$\begin{array}{l}\text { Studien- } \\
\text { design }\end{array}$} & \multirow{2}{*}{ Nikotindosis } & \multicolumn{5}{|c|}{ Nach Nikotingabe } \\
\hline & & & $\begin{array}{l}\text { REM- } \\
\text { Parameter }\end{array}$ & NREM-Schlaf & TST & $\begin{array}{l}\text { Schlaf- } \\
\text { Latenz }\end{array}$ & $\begin{array}{l}\text { - Schlaf- } \\
\text { Effizien }\end{array}$ \\
\hline Davila et al. 1994 & $a$ & $\begin{array}{l}\text { Nikotinpflaster (11 mg } \\
\text { Nikotin/24 h) }\end{array}$ & $\begin{array}{l}\text { REM-Schlaf } \\
\downarrow\end{array}$ & & $\downarrow$ & $\uparrow$ & $\downarrow$ \\
\hline Gillin et al. 1994 & $a$ & $\begin{array}{l}\text { Nikotinpflaster (7 oder } \\
14 \text { mg Nikotin/24 h) }\end{array}$ & $\begin{array}{l}\text { REM-Schlaf } \\
\downarrow\end{array}$ & Stadium $2 \uparrow$ & & & \\
\hline $\begin{array}{l}\text { Salín-Pascual et al. } \\
1995\end{array}$ & $a$ & $\begin{array}{l}\text { Nikotinpflaster } \\
(17,5 \mathrm{mg} \text { Nikotin/24 h) }\end{array}$ & $\begin{array}{l}\text { REM-Schlaf } \\
\downarrow ; \text { REM- } \\
\text { Latenz } \downarrow\end{array}$ & Stadium $1 \downarrow$ & $\downarrow$ & & \\
\hline $\begin{array}{l}\text { Salín-Pascual, } \\
\text { Drucker-Colín } \\
1998^{c}\end{array}$ & $b$ & $\begin{array}{l}\text { Nikotinpflaster } \\
(17,5 \mathrm{mg} \text { Nikotin/24 h) }\end{array}$ & $\begin{array}{l}\text { REM-Schlaf } \\
\uparrow(\text { ab PSG 2) }\end{array}$ & $\begin{array}{l}\text { Stadium } 2 \downarrow \text { (ab } \\
\text { PSG 2); Stadium } 3 \downarrow \\
\text { (PSG 1-3) }\end{array}$ & & & \\
\hline
\end{tabular}

${ }^{a}$ Doppelblind, randomisiert, placebokontrolliert, Cross-over. ${ }^{b}$ Kontrolliert. ${ }^{c} 4$ Nächte PSG. 
appliziert wurden. Es zeigte sich eine dosisabhängige Reduktion der REM-Schlafzeit und des prozentualen Anteils des REM-Schlafs, ein Anstieg des prozentualen Anteils des NREM-Schlafstadiums 2 und ein früheres Erwachen der Probanden. R. Salín-Pascual veröffentlichte zwei Studien mit ähnlichem Design. In beiden Studien wurde den Probanden zwei Stunden vor den PSG 17,5 mgNikotinpflaster appliziert. In der Studie von 1995 wurde lediglich eine Nacht mit Nikotinpflastern untersucht, in der Arbeit von 1998 hingegen vier Nächte. In beiden Veröffentlichungen wurde auch die Absetznacht ohne Nikotin beobachtet. In der Studie Salín-Pascual et al. (1995) zeigte sich akut eine Reduktion der REM-Schlafzeit, der REM-Latenz, der Gesamtschlafzeit und der Zeit im NREM-Schlafstadium 1. Die Studie drei Jahre später (Salín-Pascual und Drucker-Colín, 1998) erbrachte andere Ergebnisse. Hier konnte ab der zweiten Nacht eine erhöhte REM-Schlafzeit und eine geringere Zeit im NREM-Schlafstadium 2 beobachtet werden. Die Zeit im NREM-Schlafstadium 3 war in der ersten bis dritten Nacht vermindert. In beiden Studien sank der Punktwert des auch bei den gesunden Probanden durchgeführte Hamilton Rating Scale for Depression (HAM-D) unter Verwendung von Nikotinpflastern.

Die Forschungsgruppe um R. Salín-Pascual hat in drei Veröffentlichungen (siehe Tabelle 3.10) auch die erste Absetznacht nach der Nikotingabe polysomnografisch untersucht.

Gillin et al. (1994) stellten in der Absetznacht einen Anstieg der REM-Schlafzeit und des prozentualen Anteils des REM-Schlafs fest. Die Zeit im NREM-Schlafstadium 2 verringerte sich hingegen und es kam zu einem Abfall der REM-Latenz und -Dichte. Bei Salín-Pascual et al. (1995) zeigte sich in der Absetznacht eine weiterhin verminderte REM-Latenz. Bei Salín-Pascual und Drucker-Colín (1998) wurde in der Absetznacht nach der viertägigen Nikotingabe ein Anstieg der REM-Schlafzeit beobachtet, der höher als unter Nikotin ausfiel. Des Weiteren war die Zeit im NREM-Schlafstadium 2 nach wie vor vermindert.

Zusammenfassend kann festgehalten werden, dass Nikotin in drei von vier Studien aus zwei Forschungsgruppen die REM-Schlafzeit reduzierte und die Parameter der NREM-Schlafstadien 3 und 4 nicht beeinflusste. Ebenso war zum Großteil die REM- oder Schlaf-Latenz nicht verändert. Die Gesamtschlafzeit war in zwei Studien vermindert.

In der Absetznacht nach einer Nikotingabe konnten keine Veränderungen der Tiefschlaf- oder der Gesamtschlafzeit beobachtet werden. Ansonsten ist kein gemeinsamer Trend für den Verlauf 
Tabelle 3.10: Auswirkungen eines Nikotinentzugs auf polysomnografische Parameter bei gesunden Nichtrauchern.

\begin{tabular}{|c|c|c|c|c|c|c|}
\hline \multirow[t]{2}{*}{ Autoren } & \multirow[t]{2}{*}{$\begin{array}{l}\text { Studien- } \\
\text { design }\end{array}$} & \multirow[t]{2}{*}{ Nikotindosis } & \multirow[t]{2}{*}{$\begin{array}{l}\text { Entzugs- } \\
\text { zeit }\end{array}$} & \multicolumn{3}{|c|}{ Im Entzug } \\
\hline & & & & $\begin{array}{l}\text { REM- } \\
\text { Parameter }\end{array}$ & $\begin{array}{l}\text { NREM- } \\
\text { Schlaf }\end{array}$ & TST \\
\hline Gillin et al. 1994 & $a$ & $\begin{array}{l}\text { Nikotinpflaster (7 } \\
\text { oder } 14 \text { mg Nikotin/24 h) }\end{array}$ & $24 \mathrm{~h}$ & $\begin{array}{l}\text { REM-Schlaf } \uparrow ; \\
\text { REM-Latenz } \downarrow \text {; } \\
\text { REM-Dichte } \downarrow\end{array}$ & Stadium $2 \downarrow$ & $\begin{array}{l}\uparrow(24 \mathrm{~h} \text { nach } \\
14 \mathrm{mg})\end{array}$ \\
\hline $\begin{array}{l}\text { Salín-Pascual et al. } \\
1995\end{array}$ & $a$ & $\begin{array}{l}\text { Nikotinpflaster }(17,5 \mathrm{mg} \\
\text { Nikotin/24 h) }\end{array}$ & $24 \mathrm{~h}$ & REM-Latenz $\downarrow$ & & \\
\hline $\begin{array}{l}\text { Salín-Pascual, } \\
\text { Drucker-Colín 1998 }\end{array}$ & $b$ & $\begin{array}{l}\text { Nikotinpflaster }(17,5 \mathrm{mg} \\
\text { Nikotin/24 h) }\end{array}$ & $24 \mathrm{~h}$ & REM-Schlaf $\uparrow$ & Stadium $2 \downarrow$ & \\
\hline
\end{tabular}

${ }^{a}$ Doppelblind, randomisiert, placebokontrolliert, Cross-over. ${ }^{b}$ Kontrolliert. ${ }^{c} 4$ Nächte PSG.

polysomnografischer Parameter zu erkennen.

\section{Depressive Nichtraucher}

Die Forschungsgruppe von R. Salín-Pascual verabreichte in ihren Studien auch depressiven Probanden Nikotin und untersuchte mögliche Veränderungen polysomnografischer Parameter. Tabelle 3.11 führt die drei Studien auf, in denen die akute Reaktion auf Nikotin untersucht wurde.

Die Methodik ähnelt sich bei den drei Veröffentlichungen sehr, so wurden den depressiven Probanden in allen drei Studien Nikotinpflaster mit der Dosis von 17,5 mg Nikotin appliziert und zwei Stunden später PSG durchgeführt. In der Arbeit von Salín-Pascual und Drucker-Colín (1998) wurden vier aufeinander folgende Nächte untersucht.

Die Ergebnisse von Salín-Pascual et al. (1995) konnten von Salín-Pascual (2002) z. T. bestätigt werden, in beiden Untersuchungen stieg die REM-Schlafzeit der Probanden an und die Depressionssymptomatik besserte sich. In der Studie von 2002 kam es zusätzlich zu einem Abfall der Zeit im NREM-Schlafstadium 2. Auch bei Salín-Pascual und Drucker-Colín (1998) wurde ein Anstieg der REM-Schlafzeit und eine Besserung der Depressionen beobachtet. Eine einzige Studie verfolgte eine längerfristige Nikotingabe über acht Monate. Haro und Drucker-Colín (2004) (Tabelle 3.12) applizierten den depressiven Probanden 17,5 mg-Nikotinpflaster in folgender Dosierung: Fünf 
Tabelle 3.11: Auswirkung einer akuten Nikotingabe auf polysomnografische Parameter bei depressiven Nichtrauchern.

\begin{tabular}{|c|c|c|c|c|}
\hline \multirow[t]{2}{*}{ Autoren } & \multirow[t]{2}{*}{ Studiendesign } & \multirow[t]{2}{*}{ Nikotindosis } & \multicolumn{2}{|c|}{ Nach Nikotingabe } \\
\hline & & & REM-Parameter & NREM-Schlaf \\
\hline $\begin{array}{l}\text { Salín-Pascual et } \\
\text { al. } 1995\end{array}$ & $\begin{array}{l}\text { Doppelblind, randomisiert, } \\
\text { placebokontrolliert, Cross-over }\end{array}$ & $\begin{array}{l}\text { Nikotinpflaster } \\
(17,5 \mathrm{mg} \text { Nikotin/24 h) }\end{array}$ & REM-Schlaf $\uparrow$ & \\
\hline $\begin{array}{l}\text { Salín-Pascual, } \\
\text { Drucker-Colin } \\
1998^{a}\end{array}$ & Kontrolliert & $\begin{array}{l}\text { Nikotinpflaster } \\
(17,5 \mathrm{mg} \text { Nikotin/24 h) }\end{array}$ & $\begin{array}{l}\text { REM-Schlaf } \uparrow \\
\text { (außer PSG 2) }\end{array}$ & \\
\hline $\begin{array}{l}\text { Salín-Pascual } \\
2002\end{array}$ & Nicht kontrolliert & $\begin{array}{l}\text { Nikotinpflaster } \\
(17,5 \mathrm{mg} \text { Nikotin/24 h) }\end{array}$ & REM-Schlaf $\uparrow$ & Stadium $2 \downarrow$ \\
\hline
\end{tabular}

${ }^{a} 4$ Nächte PSG.

Tage pro Woche über sechs Monate, in Monat sieben drei Tage pro Woche und in Monat acht ein Tag pro Woche. In Monat fünf zeigte sich ein Anstieg der REM-Latenz, der Gesamtschlafzeit, der Zeit in den NREM-Schlafstadien 3 und 4 und der Schlaf-Effizienz und eine geringere Zeit im NREM-Schlafstadium 1. Die REM-Schlafzeit veränderte sich nicht. Die mittels HAM-D bestimmte Depressionssymptomatik besserte sich.

Tabelle 3.12: Auswirkung einer chronischen Nikotingabe auf polysomnografische Parameter bei depressiven Nichtrauchern.

\begin{tabular}{|c|c|c|c|c|c|c|}
\hline \multirow[t]{2}{*}{ Autoren } & \multirow[t]{2}{*}{ Studiendesign } & \multirow[t]{2}{*}{ Nikotindosis } & \multicolumn{4}{|c|}{ Nach 5 Monaten Nikotingabe } \\
\hline & & & $\begin{array}{l}\text { REM- } \\
\text { Parameter }\end{array}$ & NREM-Schlaf & TST & $\begin{array}{l}\text { Schlaf- } \\
\text { Effizienz }\end{array}$ \\
\hline $\begin{array}{l}\text { Haro, } \\
\text { Drucker- } \\
\text { Colín } \\
2004\end{array}$ & $\begin{array}{l}\text { Nicht } \\
\text { kontrolliert, } \\
\text { einfachblind }\end{array}$ & $\begin{array}{l}\text { Nikotinpflaster }(17,5 \mathrm{mg} \\
\text { Nikotin/24 h; } 5 \mathrm{~d} / \text { Woche in } \\
\text { Monat } 1-6,3 \mathrm{~d} / \text { Woche in } \\
\text { Monat } 7,1 \mathrm{~d} / \text { Woche in Monat } 8)\end{array}$ & $\begin{array}{l}\text { REM- } \\
\text { Latenz } \\
\uparrow\end{array}$ & $\begin{array}{l}\text { Stadium } 1 \downarrow ; \\
\text { Stadium } 3 \text { und } \\
4 \uparrow\end{array}$ & $\uparrow$ & $\uparrow$ \\
\hline
\end{tabular}

Auch in den Studien mit depressiven Probanden wurden z. T. die Absetznächte untersucht. Die Ergebnisse sind in Tabelle 3.13 ersichtlich.

Salín-Pascual et al. (1995) konnten den unter Nikotin beobachteten Anstieg der REM-Schlafzeit in der Absetznacht nicht mehr feststellen - im Gegensatz zu Salín-Pascual und Drucker-Colín (1998), die auch in der Absetznacht nach vier Tagen Nikotin den Anstieg der REM-Schlafzeit weiterhin beobachten konnten. Haro und Drucker-Colín (2004), die in ihrer Langzeitstudie die 
Tabelle 3.13: Auswirkung eines Nikotinentzugs auf polysomnografische Parameter bei depressiven Nichtrauchern.

\begin{tabular}{|c|c|c|c|c|c|c|c|}
\hline \multirow[t]{2}{*}{ Autoren } & \multirow[t]{2}{*}{ Studiendesign } & \multirow[t]{2}{*}{ Nikotindosis } & \multirow[t]{2}{*}{ Entzugsz } & \multicolumn{4}{|c|}{ Im Entzug: } \\
\hline & & & & $\begin{array}{l}\text { REM- } \\
\text { Parameter }\end{array}$ & $\begin{array}{l}\text { NREM- } \\
\text { Schlaf }\end{array}$ & TST & $\begin{array}{l}\text { Schlaf- } \\
\text { Effizien }\end{array}$ \\
\hline $\begin{array}{l}\text { Salín-Pascual et } \\
\text { al. } 1995\end{array}$ & $\begin{array}{l}\text { Doppelblind, } \\
\text { randomisiert, } \\
\text { placebokon- } \\
\text { trolliert, } \\
\text { Cross-over }\end{array}$ & $\begin{array}{l}\text { Nikotinpflaster (17,5 mg } \\
\text { Nikotin/24 h) }\end{array}$ & $24 \mathrm{~h}$ & $\begin{array}{l}\text { REM-Schlaf } \\
\leftrightarrow\end{array}$ & & & \\
\hline $\begin{array}{l}\text { Salín-Pascual, } \\
\text { Drucker-Colín } \\
1998^{a}\end{array}$ & Kontrolliert & $\begin{array}{l}\text { Nikotinpflaster (17,5 mg } \\
\text { Nikotin/24 h) }\end{array}$ & $24 \mathrm{~h}$ & $\begin{array}{l}\text { REM-Schlaf } \\
\uparrow\end{array}$ & & & \\
\hline $\begin{array}{l}\text { Haro, } \\
\text { Drucker-Colín } \\
2004\end{array}$ & $\begin{array}{l}\text { Nicht } \\
\text { kontrolliert, } \\
\text { einfachblind }\end{array}$ & $\begin{array}{l}\text { Nikotinpflaster }(17,5 \mathrm{mg} \\
\text { Nikotin/24 h; } 5 \mathrm{~d} / \text { Woche } \\
\text { in Monat } 1-6,3 \mathrm{~d} / \text { Woche } \\
\text { in Monat } 7,1 \mathrm{~d} / \text { Woche } \\
\text { in Monat } 8) \text {, Monat } 9-24 \\
\text { Placebo }\end{array}$ & $\begin{array}{l}15 \\
\text { Monate }\end{array}$ & $\begin{array}{l}\text { REM-Schlaf } \\
\downarrow \text { (bis } \\
\text { Monat 18); } \\
\text { REM- } \\
\text { Latenz } \uparrow\end{array}$ & $\begin{array}{l}\text { Stadium } 1 \\
\downarrow \text {; Stadi- } \\
\text { um } 3 \\
\text { und } 4 \uparrow\end{array}$ & $\uparrow$ & $\uparrow$ \\
\hline
\end{tabular}

$\overline{a^{a}} 4$ Nächte PSG.

depressiven Probanden nach acht Monaten Nikotingabe noch weitere 16 Monate polysomnografisch untersuchten und Placebopflaster einsetzten, konnten folgendes feststellen: Mit Ausnahme der REM-Schlafzeit zeigten sich die Veränderungen, die in Monat fünf unter Nikotin beobachtet wurden, auch noch in den Monaten 10-24. War die REM-Schlafzeit in Monat fünf gegenüber den Anfangswerten unverändert, fiel der Wert während der Monate 10-18 ab. Die Depressionssymptomatik der Teilnehmer war auch unter Verwendung der Placebopflaster weiterhin gebessert.

Zusammenfassend stellte man in den Studien, die eine akute Nikotingabe an depressive Probanden untersuchten, einen Anstieg der REM-Schlafzeit und ansonsten wenig beeinflusste polysomnografische Parameter fest. Eine einzige Studie unternahm einen Langzeitversuch und konnte unter mehrmonatiger Nikotingabe keine Veränderung der REM-Schlafzeit, jedoch eine Zunahme der REM-Latenz, der Zeit in den NREM-Schlafstadien 3 und 4 sowie der Gesamtschlafzeit beobachten. In allen Studien besserten sich unter Nikotin die Depressionen der Probanden.

Drei Studien beschäftigten sich auch mit den Absetznächten nach einer Nikotingabe. Auch an dieser Stelle konnten keine Veränderungen der Tiefschlafzeit oder der Gesamtschlafzeit beobachtet werden. Die Ergebnisse für den REM-Schlaf sind nicht einheitlich. Im Langzeitversuch zeigte sich 
in der Entzugszeit ein Abfall der REM-Schlafzeit, ansonsten wurden dieselben Ergebnisse wie unter Nikotin beobachtet.

\section{Zusammenfassung}

Tabelle 3.14 und 3.15 fasst die Ergebnisse der Studien zusammen, die sich mit gesunden und depressiven Nichtrauchern beschäftigt haben.

\section{Aktigrafie}

Tabelle 3.16 zeigt die einzige Studie, die bei Nichtrauchern mittels Aktigrafie die Auswirkungen einer Nikotingabe auf die Aktivität im Schlaf ermittelte.

Gehricke et al. (2009) verabreichten 27 Probanden mit der Aufmerksamkeitsdefizit-Hyperaktivitätsstörung (ADHS) zwei Tage Nikotin über 7 mg-Nikotinpflaster. Die mittels Aktigrafen gemessene Aktivität im Schlaf und am Tag unterschied sich nicht zwischen Nikotin- und Placebobedingung.

\subsubsection{Nikotinentzug bei Gelegenheitsrauchern}

Die Suche erbrachte zwei Suchergebnisse. Beide Studien erfüllen nicht die Suchkriterien.

Tabelle 3.14: Auswirkungen einer Nikotingabe auf polysomnografische Parameter gesunder Nichtraucher (Zusammenfassung).

\begin{tabular}{|c|c|c|c|c|c|}
\hline & \multirow{2}{*}{$\begin{array}{l}\text { Studien- } \\
\text { anzahl }\end{array}$} & \multirow[t]{2}{*}{ Einheitlichkeit $^{a}$} & \multicolumn{3}{|c|}{ Ergebnisse } \\
\hline & & & REM-Schlaf & NREM-Schlafstadium 3 und 4 & TST \\
\hline 1-4 d Nikotin & 4 & überwiegend & $\downarrow$ & $\leftrightarrow$ & $\leftrightarrow \downarrow$ \\
\hline Entzug & 3 & überwiegend & $\uparrow \leftrightarrow$ & $\leftrightarrow$ & $\leftrightarrow$ \\
\hline
\end{tabular}

${ }^{a}$ Mindestens $70 \%$ der Studien haben ähnliche Ergebnisse. 
Tabelle 3.15: Auswirkungen einer Nikotingabe auf polysomnografische Paramter depressiver Nichtraucher (Zusammenfassung).

\begin{tabular}{llllll}
\hline & \multicolumn{2}{l}{$\begin{array}{l}\text { Studien- Einheitlichkeit } \\
\text { anzahl }\end{array}$} & & \multicolumn{2}{c}{ Ergebnisse } \\
\cline { 3 - 6 } & & & REM-Schlaf & NREM-Schlafstadium 3 und 4 & TST \\
\hline 1-4 d Nikotin & 3 & ja & $\uparrow$ & $\leftrightarrow$ & $\leftrightarrow$ \\
Kurzzeit-Entzug & 2 & überwiegend & $\leftrightarrow \uparrow$ & $\leftrightarrow$ & $\leftrightarrow$ \\
8 Monate Nikotin & 1 & & $\leftrightarrow$ & $\uparrow$ & $\uparrow$ \\
Langzeit-Entzug & 1 & & $\downarrow$ & $\uparrow$ & $\uparrow$ \\
\hline
\end{tabular}

${ }^{a}$ Mindestens $70 \%$ der Studien haben ähnliche Ergebnisse.

Tabelle 3.16: Auswirkungen einer Nikotingabe auf die Aktivität im Schlaf bei Nichtrauchern.

\begin{tabular}{llll}
\hline Autoren & Studiendesign & Nikotindosis & $\begin{array}{l}\text { Aktivität } \\
\text { nach } \\
\text { Nikotingabe }\end{array}$ \\
\hline Gehricke et al. 2009 & $\begin{array}{l}\text { Doppelblind, randomisiert, } \\
\text { placebokontrolliert, Cross-over }\end{array}$ & $\begin{array}{l}\text { Nikotinpflaster (7 mg } \\
\text { Nikotin/24h) }\end{array}$ & $\leftrightarrow$ \\
\hline
\end{tabular}

\subsubsection{Nikotinentzug bei Rauchern}

Die Suche ergab 401 Suchergebnisse, von denen zehn den Suchkriterien entsprechen und in den Ergebnisteil aufgenommen worden sind. Fünf Forschungsgruppen haben sich in der Vergangenheit mit den polysomnografischen Folgen eines Nikotinentzugs bei Raucher beschäftigt. Die Studien sind über einen großen Zeitraum hinweg veröffentlicht worden, so stammt die älteste aus dem Jahr 1969, die neuste aus dem Jahr 2007. Zwei Forschungsgruppen haben jeweils drei Studien zu den Ergebnissen beigetragen. Dies sind die Gruppen um D. Wetter und um J. Kales. Eine weitere Forschungsgruppe hat sich 1996 mit den Veränderungen der Aktivität im Schlaf bei Rauchern im Nikotinentzug auseinandergesetzt, die mittels Aktigrafie erfasst wurde.

Um eine bessere Vergleichbarkeit zu erhalten, werden die Studien nach der Länge des Nikotinentzugs eingeteilt und besprochen. Die aktigrafische Studie wird nach den polysomnografischen Studien dargestellt. 


\section{Auswirkungen eines mehrtägigen Nikotinentzugs}

Tabelle 3.17 führt die ersten beiden Studien auf, die einen zwei- bzw. dreitägigen Nikotinentzug polysomnografisch untersucht haben.

Prosise et al. (1994) stellten während eines dreitägigen Nikotinentzugs eine erhöhte Fragmentierung des Schlafs, einhergehend mit einer größeren Anzahl an Stadienwechseln und Arousals fest. Tagsüber zeigte sich eine verstärkte Tagesmüdigkeit. Parsons und Hamme (1976) beobachteten hingegen während eines 24- bzw. 48-stündigen Entzugs einen erhöhten Anteil an Tiefschlafstadien.

In Tabelle 3.18 finden sich Studien, die einen Nikotinentzug über fünf bzw. sieben Tage begleitet haben. Soldatos et al. (1980) beobachteten einen Nikotinentzug über fünf und zwölf Tage, die Ergebnisse hierzu sind im Folgenden zeitlich getrennt dargestellt.

Kales et al. $(1969,1971)$ konnten während eines fünf- und siebentägigen Nikotinentzugs einen Anstieg der REM-Schlafzeit und des prozentualen Anteils an REM-Schlaf feststellen, zudem in der Studie von 1969 einen Abfall der REM-Latenz. Während der ersten fünf Entzugstage konnten Soldatos et al. (1980) keine Änderungen von Parametern des REM- oder NREM-Schlafs beobachten, jedoch eine geringere Schlaf-Latenz und Wachzeit. Wetter et al. $(1995,1999)$ führten zwei Untersuchungen mit derselben Methodik durch, erhielten aber z. T. unterschiedliche Ergebnisse: War unter Verwendung von Placebopflastern in beiden Studien keine Änderungen der REM- und NREM-Schlafvariablen, der Schlaf-Latenz oder der Gesamtschlafzeit aufgefallen, zeigte sich bei Wetter et al. (1995) unter Nikotingabe ein Abfall des prozentualen Anteils an NREM-Schlafstadium

Tabelle 3.17: Auswirkung eines mehrtägigen Nikotinentzugs auf polysomnografische Parameter bei Rauchern 1.

\begin{tabular}{llllll}
\hline Autoren & Studiendesign & Entzugsdauer & \multicolumn{2}{c}{ Während des Entzugs } & \\
\cline { 4 - 6 } & & & $\begin{array}{l}\text { REM- } \\
\text { Parameter }\end{array}$ & NREM-Schlaf & $\begin{array}{l}\text { Schlaf- Arousals } \\
\text { Latenz }\end{array}$ \\
\hline Parsons, Hamme 1976 & $\begin{array}{l}\text { Nicht } \\
\text { kontrolliert }\end{array}$ & $2 \mathrm{~d}$ & & $\begin{array}{l}\text { Stadium 3 und } \\
4 \uparrow\end{array}$ & $\downarrow$ \\
Prosise et al. 1994 & $\begin{array}{l}\text { Nicht } \\
\text { kontrolliert }\end{array}$ & $3 \mathrm{~d}$ & & & $\uparrow$ \\
& & & & \\
\hline
\end{tabular}


Tabelle 3.18: Auswirkung eines mehrtägigen Nikotinentzugs auf polysomnografische Parameter bei Rauchern 2.

\begin{tabular}{|c|c|c|c|c|c|c|c|}
\hline \multirow[t]{2}{*}{ Autoren } & \multirow[t]{2}{*}{ Studiendesign } & \multirow{2}{*}{$\begin{array}{l}\text { Entzugs- } \\
\text { dauer }\end{array}$} & \multicolumn{5}{|c|}{ Während des Entzugs } \\
\hline & & & $\begin{array}{l}\text { REM- } \\
\text { Parameter }\end{array}$ & $\begin{array}{l}\text { NREM- } \\
\text { Schlaf }\end{array}$ & $\begin{array}{l}\text { Schlaf- } \\
\text { Latenz }\end{array}$ & $\begin{array}{l}\text { Schlaf- } \\
\text { Effizienz }\end{array}$ & $\begin{array}{l}\text { Arousals, } \\
\text { WZ }\end{array}$ \\
\hline $\begin{array}{l}\text { Kales et al. } \\
1969\end{array}$ & Nicht kontrolliert & $5 \mathrm{~d}$ & $\begin{array}{l}\text { REM-Schlaf } \uparrow ; \\
\text { REM-Latenz } \downarrow\end{array}$ & & & & \\
\hline $\begin{array}{l}\text { Soldatos } \\
\text { et al. } 1980\end{array}$ & Nicht kontrolliert & $5 \mathrm{~d}$ & & & $\downarrow$ & & $\mathrm{WZ} \downarrow$ \\
\hline $\begin{array}{l}\text { Wetter et } \\
\text { al. } 1995\end{array}$ & $\begin{array}{l}\text { Doppelblind, } \\
\text { randomisiert, pla- } \\
\text { cebokontrolliert }\end{array}$ & $5 \mathrm{~d}$ & & $\begin{array}{l}\text { Stadium } 2 \downarrow^{a} \text {; } \\
\text { Stadium } 3 \\
\text { und } 4 \uparrow^{a}\end{array}$ & & & $\begin{array}{l}\text { Arousals } \uparrow, \\
\text { Arousals } \downarrow^{a}\end{array}$ \\
\hline $\begin{array}{l}\text { Wetter et } \\
\text { al. } 1999\end{array}$ & $\begin{array}{l}\text { Doppelblind, } \\
\text { randomisiert, pla- } \\
\text { cebokontrolliert }\end{array}$ & $5 \mathrm{~d}$ & & & & $\begin{array}{l}\uparrow(\text { nur w), } \\
\downarrow(\text { nur w) }\end{array}$ & $\begin{array}{l}\mathrm{WZ} \downarrow, \mathrm{WZ} \downarrow \\
(\text { nur m })^{a}, \mathrm{WZ} \\
\uparrow(\text { nur w) }\end{array}$ \\
\hline $\begin{array}{l}\text { Kales et al. } \\
1971\end{array}$ & Nicht kontrolliert & $7 \mathrm{~d}$ & REM-Schlaf $\uparrow$ & & & & \\
\hline
\end{tabular}

${ }^{a}$ Mit Nikotinpflaster (22 mg Nikotin/24 h).

2 und ein Anstieg der prozentualen Anteile der NREM-Schlafstadien 3 und 4. Zudem fiel unter Verwendung von Nikotinpflastern eine geringere Anzahl von Arousals auf. Wetter et al. (1999), die die Resultate auch geschlechtsspezifisch darstellten, konnten einen Unterschied zwischen Männern und Frauen ausmachen: Männer, die Nikotinpflaster trugen, zeigten eine geringere Fragmentierung des Schlafs und profitierten somit von der Nikotinersatztherapie - im Gegensatz zu den Frauen, die unter Nikotinpflastern eine geringere Schlaf-Effizienz aufwiesen. In beiden Studien war der Schlaf nach Applikation von Nikotinpflastern - zumindest bei den männlichen Probanden - objektiv verbessert, jedoch wurde dies von den Probanden subjektiv nicht wahrgenommen.

Tabelle 3.19 beinhaltet zwei Studien, in denen die Probanden über zehn bzw. zwölf Tage untersucht wurden. Die Studie von Wetter et al. (2000) beschäftigte sich neben gesunden auch mit depressiven Raucherinnen.

Soldatos et al. (1980) konnten am elften und zwölften Entzugstag keine Veränderungen bezüglich der Baseline-Messungen mehr ausmachen. Betrachtet man die Studie von Wetter et al. (2000), wurde für beide Probandengruppen im Nikotinentzug eine Abnahme der Gesamtschlafzeit und eine erhöhte Fragmentierung des Schlafs in der dritten Entzugsnacht festgestellt. Ebenso war 
Tabelle 3.19: Auswirkung eines mehrtägigen Nikotinentzugs auf polysomnografische Parameter bei Rauchern 3.

\begin{tabular}{|c|c|c|c|c|c|}
\hline \multirow[t]{2}{*}{ Autoren } & \multirow[t]{2}{*}{ Studiendesign } & \multirow[t]{2}{*}{ Entzugsdauer } & \multicolumn{3}{|c|}{ Während des Entzugs } \\
\hline & & & $\begin{array}{l}\text { REM- } \\
\text { Parameter }\end{array}$ & NREM-Schlaf & WZ, TST \\
\hline $\begin{array}{l}\text { Soldatos et al. } \\
1980\end{array}$ & $\begin{array}{l}\text { Nicht } \\
\text { kontrolliert }\end{array}$ & $12 \mathrm{~d}$ & $\leftrightarrow$ & $\leftrightarrow$ & $\leftrightarrow$ \\
\hline $\begin{array}{l}\text { Wetter et al. } \\
2000\end{array}$ & Kontrolliert & $10 \mathrm{~d}$ & $\begin{array}{l}\text { REM-Latenz } \uparrow \\
\text { (Nacht } 3 \text { und } \\
5 \text { ), } \downarrow \\
\text { (Nacht 10) }\end{array}$ & $\begin{array}{l}\text { Stadium } 1^{a} \uparrow(\text { Nacht } 3), \downarrow \\
\text { (Nacht } 5 \text { und } 10) ; \\
\text { Stadium } 3^{a} \text { und } 4^{a} \downarrow \\
\text { (Nacht } 3), \uparrow(\text { Nacht } 5 \text { und } 10)\end{array}$ & $\begin{array}{l}\text { WZ } \uparrow \text { (Nacht } 3) ; \\
\mathrm{TST}^{a} \downarrow\end{array}$ \\
\hline
\end{tabular}

${ }^{a}$ Gilt auch für depressive Probanden.

der prozentuale Anteil an Tiefschlaf in dieser Entzugsphase vermindert, stieg jedoch bis Nacht zehn wieder an. Die objektive Verschlechterung der Schlafqualität wurde von den Probandinnen auch subjektiv empfunden. Die depressiven Teilnehmerinnen unterschieden sich bezüglich der polysomnografischen Parameter lediglich in den REM-Schlafvariablen. Bei den depressiven Probandinnen stieg der prozentuale Anteil an REM-Schlaf im Nikotinentzug nicht signifikant an, bei den gesunden veränderte er sich nicht. Die REM-Latenz blieb hingegen in der depressiven Probandengruppe unverändert, stieg aber bei den gesunden Teilnehmerinnen bis Nacht fünf an, um in der zehnten Nacht wieder abzufallen.

Zusammenfassend wurden in den ersten Entzugstagen zu einem großen Teil keine Änderungen der prozentualen Anteile an REM- oder Tiefschlaf oder eine veränderte Gesamtschlafzeit beobachtet. Zwei Forschungsgruppen stellten eine erhöhte Fragmentierung des Schlafs in der dritten Entzugsnacht fest. Die Forschungsgruppe um D. Wetter konnte eine Verbesserung der Schlafqualität im Entzug durch den Einsatz von Nikotinpflastern zeigen.

\section{Auswirkungen eines Langzeit-Nikotinentzugs}

Tabelle 3.20 zeigt die einzige Studie (Moreno-Coutiño et al., 2007), die sich mit einem längerfristigen Nikotinentzug bei Rauchern auseinandergesetzt hat. Über einen Zeitraum von zwölf Monaten wurden PSG durchgeführt und folgende Veränderungen der Schlafparameter beobachtet:

Der prozentuale REM-Schlafanteil war im vierten, sechsten und neunten Monat erhöht und die 
Tabelle 3.20: Auswirkung eines Langzeit-Nikotinentzugs auf polysomnografische Parameter bei Rauchern.

\begin{tabular}{|c|c|c|c|c|}
\hline \multirow[t]{2}{*}{ Autoren } & \multirow[t]{2}{*}{ Studiendesign } & \multirow[t]{2}{*}{ Entzugsdauer } & \multicolumn{2}{|c|}{ Während des Entzugs } \\
\hline & & & REM-Parameter & NREM-Schlaf \\
\hline $\begin{array}{l}\text { Moreno-Coutiño } \\
\text { et al. } 2007\end{array}$ & $\begin{array}{l}\text { Nicht } \\
\text { kontrolliert }\end{array}$ & 12 Monate & $\begin{array}{l}\text { REM-Schlaf } \uparrow \text { (Monat 4, } 6 \\
\text { und 9); REM-Latenz } \downarrow \\
\text { (außer Monat 6) }\end{array}$ & $\begin{array}{l}\text { Stadium } 1 \downarrow \text { (Monat } 2,4 \text { und 6); } \\
\text { Stadium } 2 \uparrow \text { (außer Monat 6); } \\
\text { Stadium } 3 \text { und } 4 \downarrow\end{array}$ \\
\hline
\end{tabular}

REM-Latenz verkürzte sich in dem einjährigen Entzugszeitraum mit Ausnahme des sechsten Monats. Der prozentuale Anteil an Tiefschlaf war verkürzt. Der Punktwert des HAM-D stieg im Nikotinentzug an.

\section{Zusammenfassung}

Tabelle 3.21 fasst die Ergebnisse der Studien zusammen, die sich mit den Auswirkungen eines Entzugs auf polysomnografische Parameter bei Rauchern auseinandergesetzt haben.

\section{Aktigrafie}

In Tabelle 3.22 ist die Studie aufgeführt (Wolter et al., 1996), in der mittels Aktigrafie die Aktivität der Teilnehmer im Nikotinentzug ermittelt wurde. In dieser Studie wurden auch Nikotinpflaster unterschiedlicher Dosierung eingesetzt.

Tabelle 3.21: Auswirkungen eines Nikotinentzugs auf polysomnografische Parameter bei Rauchern (Zusammenfassung).

\begin{tabular}{llllll}
\hline Dauer der Entzugs & Studienanzahl $^{*}$ & Einheitlichkeit $^{a}$ & \multicolumn{2}{c}{ Ergebnisse } & \\
\cline { 4 - 6 } & & & $\begin{array}{l}\text { REM- } \\
\text { Schlaf }\end{array}$ & $\begin{array}{l}\text { NREM-Schlafstadium } \\
\text { 3 und 4 }\end{array}$ & TST \\
\hline Tage & 8 & ja & $\leftrightarrow$ & $\leftrightarrow$ & $\leftrightarrow$ \\
Wochen & 1 & & $\uparrow$ & $\downarrow$ & $\leftrightarrow$ \\
\hline
\end{tabular}

${ }^{a}$ Mindestens $70 \%$ der Studien haben ähnliche Ergebnisse. 
Tabelle 3.22: Auswirkung eines Nikotinentzugs auf die Aktivität im Schlaf bei Rauchern.

\begin{tabular}{llll}
\hline Autoren & Studiendesign & Entzugsdauer $\begin{array}{l}\text { Aktivität während } \\
\text { des Entzugs }\end{array}$ \\
\hline Wolter et al. 1996 & Doppelblind, randomisiert, placebokontrolliert & 1 Woche & $\leftrightarrow$ \\
\hline
\end{tabular}

Weder mit unterschiedlich dosierten Nikotin- noch mit Placebopflastern wurde in der ersten Entzugswoche eine Veränderung der nächtlichen Aktivität oder der Schlaf-Effizienz beobachtet. Die Gesamtschlafzeit war jedoch vermindert. Die subjektive Einschätzung der Probanden ergab, dass eine geringere Nikotindosis mit einer schlechteren Schlafqualität verbunden war. In den folgenden Wochen wurden keine Placebopflaster mehr eingesetzt und es wurde in der achten Woche ein Unterschied in der nächtlichen Aktivität zwischen den Nikotindosen festgestellt: Probanden mit 11 mg-Nikotinpflaster zeigten gegenüber Probanden mit 22 mg-Nikotinpflaster eine bessere Schlaf-Effizienz, die mit einer geringeren Aktivität einherging. Für die am Tag gewonnenen Messwerte konnte eine positive Korrelation zwischen Nikotindosis und Aktivität gemessen werden: Je höher die Nikotindosis der Pflaster, desto höher die Aktivität am Tag. Dementsprechend wiesen die Probanden mit Placebopflastern tagsüber die geringste Aktivität auf.

\subsection{Dopaminerges System}

\subsubsection{Nikotingabe an Nichtraucher}

Die Suche erbrachte mit den verwendeten Stichwörtern 132 Suchergebnisse. Keine dieser Studien entspricht den Suchkriterien.

\subsubsection{Nikotinentzug bei Gelegenheitsrauchern}

Die Suche erbrachte mit den verwendeten Stichwörtern vier Suchergebnisse. Keine dieser Studien entspricht den Suchkriterien. 


\subsubsection{Nikotinentzug bei Rauchern}

Die Suche erbrachte mit den verwendeten Stichwörtern 847 Suchergebnisse. Fünf dieser Studien entsprechen den Suchkriterien und sind in den Ergebnisteil aufgenommen worden. Vier Forschungsgruppen haben sich mit den Auswirkungen eines Nikotinentzugs auf dopaminerge Parameter auseinandergesetzt. Bis auf eine Arbeit sind alle Veröffentlichungen nach 2001 erschienen.

In den Studien wurden drei unterschiedliche Methoden zur Bestimmung der dopaminergen Parameter verwendet. Zum einen wurde die Positronen-Emissions-Tomografie (PET) genutzt, um das Bindungspotential von Liganden an die Dopaminrezeptoren zu bestimmen. Zum anderen wurde in einer Studie die Dopaminkonzentration im 24-Stunden-Sammelurin gemessen. In einer letzten Veröffentlichung kam der Apomorphine-Challenge-Test (ACT) zur Anwendung, durch den Rückschlüsse auf die Funktion der D2-Rezeptoren möglich sind. Hierbei fungiert Apomorphin als Ligand an die genannten Rezeptoren und führt zu einer Sekretion des Wachstumshormons, welches im Blut bestimmt wird.

Folgend werden zuerst die PET-Studien dargestellt und danach die zwei verbleibenden Studien mit anderen Messtechniken.

\section{PET-Studien}

Die dargestellten Studien finden sich in Tabelle 3.23.

Tabelle 3.23: Auswirkung eines Nikotinentzugs auf das Bindungspotential an Dopaminrezeptoren bei Rauchern. ${ }^{a b}$

\begin{tabular}{|c|c|c|c|}
\hline Autoren & BP an ... & Entzugszeit & Entzug \\
\hline Dagher et al. 2001 & D1-Rezeptoren & $12 \mathrm{~h}$ & $\leftrightarrow$ \\
\hline Yasuno et al. 2007 & D1-Rezeptoren & 6 Monate & $\uparrow$ \\
\hline Fehr et al. 2008 & D2- und D3-Rezeptoren & $24 \mathrm{~h}$ & $\leftrightarrow$ \\
\hline
\end{tabular}


Dagher et al. (2001) und Yasuno et al. (2007) haben in ihren Veröffentlichungen die D1-Rezeptoren untersucht. Dagher et al. (2001) in einem Entzugszeitraum von circa zwölf Stunden, Yasuno et al. (2007) über einen Zeitraum von sechs Monaten. Nach einer Entzugszeit von zwölf Stunden konnten Dagher et al. (2001) keine Unterschiede in dem Bindungspotential der Liganden an die D1-Rezeptoren im Striatum gegenüber der Baseline-Messung feststellen. Yasuno et al. (2007) beobachteten innerhalb der sechsmonatigen Entzugszeit einen Anstieg der Ligandenbindung im Striatum. Vergleicht man das Bindungspotential an die D1-Rezeptoren vor dem Entzug zwischen den rückfälligen und den abstinenten Probanden, sind keine Unterschiede festzustellen. Fehr et al. (2008) bestimmten das Bindungspotential an die D2- und D3-Rezeptoren vor und nach einer Entzugszeit von 24 Stunden. Wie bei Dagher et al. (2001) wurden keine Änderungen des Bindungspotentials der Liganden gefunden.

\section{Andere Studien}

Tabelle 3.24 und 3.25 zeigen zwei Studien, die mithilfe anderer Methoden als der PET dopaminerge Parameter im Nikotinentzug untersucht haben.

Tabelle 3.24 führt die Veröffentlichung von Ward et al. (1991). Sie bestimmten während eines 30tägigen Entzugs die Dopaminkonzentration im 24-Stunden-Sammelurin der abstinenten Raucher. Diese fiel während des beobachteten Zeitraums ab - nur am dritten Entzugstag konnten ähnliche Werte wie vor dem Entzug gemessen werden.

Tabelle 3.24: Auswirkung eines Nikotinentzugs auf die Dopaminkonzentration im Urin bei Rauchern.

\begin{tabular}{llll}
\hline Autoren & Studiendesign & Entzugszeit & Entzug \\
\hline Ward et al. 1991 & Nicht kontrolliert & $30 \mathrm{~d}$ & $\downarrow$ (Tag 1), ↔(Tag 3), $\downarrow$ (bis Tag 30) \\
\hline
\end{tabular}

Smolka et al. (2004) verwendeten den ACT um die Funktion der D2-Rezeptoren im Nikotinentzug zu analysieren (siehe Tabelle 3.25). Hierzu verglichen sie die Apomorphin-induzierte Ausschüttung des Wachstumshormons vor und während einer zwölfstündigen Abstinenz. Es zeigten sich keine Unterschiede. Allerdings konnte eine Korrelation der Menge des ausgeschütteten Wachstumshor- 
mons mit dem Grad der Nikotinabhängigkeit festgestellt werden: Je stärker die Nikotinabhängigkeit, desto geringer die hormonelle Antwort auf den ACT.

Tabelle 3.25: Auswirkung eines Nikotinentzugs auf den ACT bei Rauchern.

\begin{tabular}{llll}
\hline Autoren & Studiendesign & Entzugszeit Entzug \\
\hline Smolka et al. 2004 & Nicht kontrolliert & $12 \mathrm{~h}$ & $\leftrightarrow$ \\
\hline
\end{tabular}

Als Resümee der Studien, die sich mit dem dopaminergen System im Nikotinentzug auseinandersetzten, kann festgehalten werden, dass in den ersten 24 Stunden des Entzugs keine wesentlichen Veränderungen stattfinden. Betrachtet man einen längerfristigen Entzug, fällt das Dopamin im 24-Stunden-Sammelurin ab und das Bindungspotential an die D1-Rezeptoren im Striatum nimmt $\mathrm{zu}$.

\subsection{Hypothalamus-Hypophysen-Nebennieren-Achse}

\subsubsection{Nikotingabe in tierexperimentellen Untersuchungen}

In dem Abschnitt der Literaturrecherche, in dem die Auswirkungen von Nikotin auf die HHN-Achse untersucht wurden, entfällt ein Großteil der gefundenen Veröffentlichungen auf tierexperimentelle Studien. Die Suche ergab 526 Suchergebnisse, von denen 37 Studien den Suchkriterien entsprechen und in den Ergebnisteil aufgenommen worden sind. Es ließen sich hierbei 26 Forschungsgruppen finden, welche sich in einem Zeitraum von 1968 bis 2012 mit der Frage auseinandergesetzt haben, welche Konsequenzen eine Nikotingabe für die HHN-Achse bei Tieren hat. Insbesondere die akuten Veränderungen, die nach einmaliger Nikotingabe entstehen, sind erforscht worden.

Nachfolgend werden die Wissenschaftler erwähnt, die mehr als eine Studie in diesem Themengebiet veröffentlicht haben. Dies sind D. Balfour, G. Cam, T. Kita, K. Andersson, B. Sharp und M. Rhodes.

Im Gegensatz zum menschlichen Organismus ist bei Nagetieren Corticosteron das wichtigste Glucocorticoid. Dementsprechend wurde in den Studien mit Nagern Corticosteron und nicht Cortisol bestimmt. Die Darstellung der Ergebnisse wird eingeteilt nach der Dauer der Nikotingabe. 
Nachdem die Studien der akuten und chronischen Gabe behandelt wurden, folgen die Studien, die auch einen Nikotinentzug bei Tieren untersucht haben.

\section{Akute Nikotingabe}

Die Tabellen 3.26-3.30 fassen die 24 Studien zusammen, die sich mit den Konsequenzen einer akuten Nikotingabe auf die Corticosteron- und ACTH-Konzentration im tierischen Organismus beschäftigt haben. Dargestellt werden die Studien chronologisch und nach Forschungsgruppen sortiert.

Die ersten fünf Studien sind in Tabelle 3.26 aufgeführt. Kershbaum et al. (1968), Turner (1975) und Balfour et al. (1975) verabreichten Ratten Nikotin und beobachteten einen Anstieg der Corticosteron-Konzentration im Blut. Kershbaum et al. (1968) und Suzuki et al. (1973) stellten dies auch nach einer Nikotingabe an Hunde fest. Nachdem den Versuchstieren bei Suzuki et al. (1973) die Hypophysen entnommen wurden, konnte der zuvor festgestellte Hormonanstieg nicht mehr beobachtet werden. Bei Altland et al. (1978) waren Ratten vier Stunden lang dem Rauch von Zigaretten ausgesetzt. Die Corticosteron-Konzentration im Blut änderte sich nicht.

Tabelle 3.26: Auswirkung einer akuten Nikotingabe auf die HHN-Achse in tierexperimentellen Untersuchungen $1 .^{a}$

\begin{tabular}{llll}
\hline Autoren & Versuchstiere & Nikotindosis & $\begin{array}{l}\text { Nach } \\
\text { Nikotingabe }\end{array}$ \\
\cline { 3 - 4 } & & & CS \\
\hline Kershbaum et al. 1968 & Hunde, Ratten & unbekannt & $\uparrow$ \\
Suzuki et al. 1973 & Hunde & $0,1 \mathrm{mg} / \mathrm{kg}$ Nikotin i. v. & $\uparrow$ \\
Balfour et al. 1975 & Ratten & $0,4 \mathrm{mg} / \mathrm{kg}$ Nikotin s. c. & $\uparrow$ \\
Turner 1975 & Ratten & $0,4 \mathrm{mg} / \mathrm{kg}$ Nikotin s. c. & $\uparrow$ \\
Altland et al. 1978 & Ratten & Zigarettenrauch-Exposition & $\leftrightarrow$ \\
\hline
\end{tabular}

${ }^{a}$ Kurzbezeichnungen: Adrenocorticotropes Hormon (ACTH), Cortisol (C), Corticosteron (CS), Tag(e) (d), Stunden (h), intraperitoneal (i.p.), intravenös (i. v.), Milligramm pro Kilogramm (mg/kg), Mikrogramm pro Kilogramm $(\mu \mathrm{g} / \mathrm{kg})$, Minuten (min), nicht signifikant (n. s.), per os (p. o.), Sprague-Dawley (SD), subkutan (s. c.), weiblich (w), signifikant erhöht $(\uparrow)$, gleichbleibend $(\leftrightarrow)$, signifikant erniedrigt $(\downarrow)$.

Tabelle 3.27 fasst die Ergebnisse der beiden Forschungsgruppen um G. Cam und K. Andersson zusammen. Alle fünf Studien weisen einen Anstieg der Blut-Corticosteron-Konzentration nach 
Nikotingabe an Ratten nach, Cam und Bassett (1983b) zudem auch einen Anstieg der ACTHKonzentration. Cam et al. (1979) hypophysektomierten die Ratten und konnten, ebenso wie Suzuki et al. (1973), keinen Anstieg der Hormonkonzentration mehr feststellen. Im Gegensatz zu Altland et al. (1978) konnten Andersson et al. (1985) nach Zigarettenrauch-Exposition einen Anstieg der Corticosteron-Konzentration beobachten.

Die Forschungsgruppe von B. Sharp veröffentlichte zwei Studien aus der Tabelle 3.28 (Sharp und Beyer, 1986; Matta et al., 1987). Nach Nikotingabe an Ratten wurde die ACTH-Konzentration im Blut bestimmt. Auch hier kam es zu Konzentrationsanstiegen des ACTHs, auch wenn das Nikotin wie bei Matta et al. (1987) intrazerebral verabreicht wurde. Weidenfeld et al. (1989) und Caggiula et al. (1991) beobachteten nach der Gabe von Nikotin einen Anstieg der Corticosteron- bzw. der ACTH-Konzentration im Blut.

Auch in den Studien der Tabelle 3.29 zeigte sich ein Anstieg der Hormonkonzentrationen des Corticosterons und des ACTHs. Gentile et al. (2011) konnten zudem zeigen, dass die weiblichen Ratten höhere Cortiocosteron- und ACTH-Werte aufwiesen als ihre männlichen Artgenossen.

In den Studien der letzten Tabelle in diesem Abschnitt (Tabelle 3.30) wurden ebenfalls ansteigende Corticosteron- und ACTH-Konzentrationen im Blut nach Nikotingabe gefunden. Loomis und Gilmour (2010) untersuchten als einzige Urin als Analysenmaterial. Nach Nikotinapplikation stieg die Konzentration des Corticosterons im Urin der Ratten an. Bei Pentkowski et al. (2011) wurde der nikotininduzierte Anstieg des Corticosterons nur bei Ratten beobachtet, die isoliert gehalten wurden.

Tabelle 3.27: Auswirkung einer akuten Nikotingabe auf die HHN-Achse in tierexperimentellen Untersuchungen 2.

\begin{tabular}{|c|c|c|c|c|}
\hline \multirow[t]{2}{*}{ Autoren } & \multirow[t]{2}{*}{ Versuchstiere } & \multirow[t]{2}{*}{ Nikotindosis } & \multicolumn{2}{|c|}{ Nach Nikotingabe } \\
\hline & & & CS & АСТН \\
\hline Cam et al. 1979 & Ratten & $0,1,0,2,0,5 \mathrm{mg} / \mathrm{kg}$ Nikotin & $\uparrow$ & \\
\hline Cam, Bassett 1983a & Ratten & $0,2 \mathrm{mg} / \mathrm{kg}$ Nikotin i. p. & $\uparrow$ & \\
\hline Cam, Bassett 1983b & Ratten & Unbekannt & $\uparrow$ & $\uparrow$ \\
\hline Andersson et al. 1985 & Ratten & Zigarettenrauch-Exposition & $\uparrow$ & \\
\hline Andersson et al. 1993 & Ratten & 0,01-1 mg/kg Nikotin i.p. & $\uparrow$ & \\
\hline
\end{tabular}


Tabelle 3.28: Auswirkung einer akuten Nikotingabe auf die HHN-Achse in tierexperimentellen Untersuchungen 3.

\begin{tabular}{lllll}
\hline Autoren & Versuchstiere & Nikotindosis & \multicolumn{2}{c}{ Nach Nikotingabe } \\
\cline { 4 - 5 } & & & CS & ACTH \\
\hline Sharp, Beyer 1986 & Ratten & $0,1-2 \mathrm{mg} / \mathrm{kg}$ Nikotin i.p. & & $\uparrow$ \\
Matta et al. 1987 & Ratten & $0,01,0,03 \mathrm{mg} / \mathrm{kg}$ Nikotin i.v. & & $\uparrow$ \\
Weidenfeld et al. 1989 & Ratten & $0,065-2,1 \mathrm{mg} / \mathrm{kg}$ Nikotin i.v. & $\uparrow$ & $\uparrow$ \\
Caggiula et al.1991 & Ratten & $0,33,0,66 \mathrm{mg} / \mathrm{kg}$ Nikotin s. c. & $\uparrow$ & \\
\hline
\end{tabular}

Tabelle 3.29: Auswirkung einer akuten Nikotingabe auf die HHN-Achse in tierexperimentellen Untersuchungen 4.

\begin{tabular}{lllll}
\hline Autoren & Versuchstiere & Nikotindosis & \multicolumn{2}{c}{ Nach Nikotingabe } \\
\cline { 4 - 5 } & & & CS & ACTH \\
\hline Okamoto et al. 1992 & Ratten & $5 \mathrm{mg} / \mathrm{kg}$ Nikotin i.p. & $\uparrow$ & \\
Mellon, Bayer 1999 & Ratten & $1 \mathrm{mg} / \mathrm{kg} \mathrm{Nikotin} \mathrm{s.} \mathrm{c.}$ & $\uparrow$ & \\
Rhodes et al. 2001 & Ratten & $0,03-0,5 \mathrm{mg} / \mathrm{kg} \mathrm{Nikotin} \mathrm{i.p.}$ & $\uparrow$ & $\uparrow$ \\
Porcu et al. 2003 & Ratten & $0,03-2 \mathrm{mg} / \mathrm{kg} \mathrm{Nikotin} \mathrm{i.p.}$ & $\uparrow$ & \\
Gentile et al. 2011 & Ratten & $0,1,03,0,5 \mathrm{mg} / \mathrm{kg}$ Nikotin & $\uparrow$ & $\uparrow$ \\
& & i. p. & & \\
Skwara et al. 2012 & Ratten & $0,3,0,5 \mathrm{mg} / \mathrm{kg}$ Nikotin i.p. & $\uparrow$ & $\uparrow$ \\
\hline
\end{tabular}

Tabelle 3.30: Auswirkung einer akuten Nikotingabe auf die HHN-Achse in tierexperimentellen Untersuchungen 5.

\begin{tabular}{lllll}
\hline Autoren & Versuchstiere & Nikotindosis & \multicolumn{2}{c}{ Nach Nikotingabe } \\
\cline { 3 - 5 } & & & CS & ACTH \\
\hline Gądek-Michalska, Bugajski 2004 & Ratten & $1-5 \mathrm{mg} / \mathrm{kg}$ Nikotin i.p. & $\uparrow$ & $\uparrow$ \\
Lutfy et al. 2006 & Mäuse & $0,02-2 \mathrm{mg} / \mathrm{kg}$ Nikotin s. c. & $\uparrow$ & $\uparrow$ \\
Cruz et al. 2008 & Ratten & $0,4 \mathrm{mg} / \mathrm{kg}$ Nikotin s. c. & $\uparrow$ & \\
Loomis, Gilmour 2010 & Ratten & $0,1-1 \mathrm{mg} / \mathrm{kg}$ Nikotin s. c. & $\uparrow$ & \\
Pentkowski et al. 2011 & Ratten & $0,6 \mathrm{mg} / \mathrm{kg}$ Nikotin s. c. & $\uparrow$ & \\
\hline
\end{tabular}

${ }^{a}$ Nur Ergebnisse isolierter Haltung betrachtet. 
Zusammenfassend zeigte sich bis auf eine Ausnahme ein Anstieg der Hormone der HHN-Achse nach akuter bzw. einmaliger Nikotingabe.

Weitere Aspekte Aus einigen Studien geht hervor, wann die maximale Hormonkonzentration nach Nikotingabe erreicht wurde und wann die Corticosteron- und ACTH-Konzentrationen wieder auf Ausgangswerte zurückkehrten. Hierbei lag die maximale Hormonkonzentration in der ersten halben Stunde nach Nikotingabe. Der Zeitpunkt, zu dem sich die Hormone wieder auf dem Niveau vor Nikotingabe befanden, variiert stark. Es finden sich Werte zwischen 30 und 120 Minuten.

Balfour et al. (1975) setzten neben Nikotin auch Metopiron, ein Hemmstoff der Cortisolsynthese, ein und bestimmten die Corticosteron-Konzentration im Blut. Eine dreitägige Metopirongabe resultierte in einem Absinken der Hormonkonzentration. Wurde nach diesen drei Tagen einmalig Nikotin gegeben, stieg die Konzentration des Corticosterons an, jedoch nicht so stark wie nach Nikotin allein.

Andersson et al. (1993) verabreichten Ratten nicht nur Nikotin, sondern auch den Nikotinmetaboliten Cotinin. Dieser konnte keinen Hormonanstieg bewirken.

Vier Veröffentlichungen bestimmten die Hormone der HHN-Achse nach Nikotingabe und Stress bzw. Laufarbeit der Ratten. Balfour et al. (1975) konnten während eines Stresstests keine Hormonunterschiede zwischen den Ratten feststellen, die vorher Nikotin oder Kochsalz appliziert bekommen hatten. In beiden Gruppen stieg die Corticosteron-Konzentration an. Allerdings stellte sich heraus, dass diese in der Nikotingruppe länger erhöht blieb. Stellten Altland et al. (1978) nach vierstündiger Zigarettenrauch-Exposition der Ratten unter Ruhebedingungen noch keine Hormonänderungen fest, zeigte sich eine Erhöhung der Corticosteron-Konzentration nach hoher ZigarettenrauchKonzentration und gleichzeitiger Laufarbeit. Bei Lutfy et al. (2006) wurde ein Teil der Mäuse fünf Tage Stress ausgesetzt und einmalig Nikotin gegeben. In beiden Gruppen stieg die ACTH- und Corticosteron-Konzentration an. Bei Mäusen, die Nikotin erhalten hatten, konnten jedoch höhere ACTH-Konzentrationen gemessen werden - die Corticosteron-Konzentration unterschied sich von der Kontrollgruppe hingegen nicht. Cruz et al. (2008) wählten einen ähnlichen Versuchsaufbau. Sie setzten Ratten sieben Tage lang Stress aus und bestimmten die Corticosteron-Konzentration im Blut nach einmaliger Nikotingabe. Ihr Ergebnis deckt sich mit dem von Lutfy et al. (2006), 
auch hier konnte kein Unterschied in der Corticosteron-Konzentration zwischen Nikotin- und Kontrollgruppe festgestellt werden. Das ACTH wurde in dieser Studie nicht analysiert.

\section{Chronische Nikotingabe}

In den Tabellen 3.31 und 3.32 sind die elf Studien aufgelistet, in denen Versuchstieren über mehrere Tage Nikotin verabreicht wurde. Eingeteilt und erläutert werden sie nach der Länge der Nikotingabe.

Bei Chen H et al. (2008) konnten sich Ratten über eine Konstruktion drei Tage lang selbst Nikotin i. v. zuführen. Die bestimmten Hormone, das ACTH und Corticosteron, stiegen nur an Tag eins an (nicht signifikant für ACTH). Davis et al. (2005) und Caggiula et al. (1991) konnten ebenfalls eine Konzentrationserhöhung des Corticosterons nach sechs bzw. zehn Tagen Nikotingabe an Ratten feststellen. Pauly et al. (1992) verabreichten Mäusen über zwölf Tage Nikotin und auch hier war das Ergebnis, dass die Corticosteron-Konzentration im Blut der Tiere anstieg (allerdings zeigte sich dies auch in der Kontrollgruppe, die Kochsalz erhielt). Dies konnte - wenn auch nicht signifikant von Morse (1989) bestätigt werden, der Kaninchen über 13 Tage Nikotin infundierte.

Zwei weitere Studien, in denen Ratten Nikotin über einen Zeitraum von 14 Tagen gegeben wurde, sind die von Faraday et al. (2005) und Chen M et al. (2007). Faraday et al. (2005) konnten nur bei den Weibchen einer bestimmten Rattenart eine Konzentrationserhöhung des ACTHs und des Corticosterons beobachten. Die Blutwerte der Männchen änderten sich nicht. Chen M et al. (2007), die, wie in den meisten Studien üblich, keine geschlechtliche Differenzierung vornahmen, stellten fest, dass die Corticosteron-Konzentration im Blut der Tiere anstieg.

Tabelle 3.32 zeigt die vier Studien, in denen ein längerer Zeitraum beobachtet wurde.

Benwell und Balfour (1979) sowie Kurogochi et al. (1985) beobachteten über eine Zeit von 40 Tagen den Verlauf der Corticosteron-Konzentration im Blut von Ratten, die jeden Tag Nikotin bekamen. Benwell und Balfour (1979) stellten eine Erhöhung dieser Hormone bis Tag fünf fest, danach kehrten die Konzentrationen auf die Ausgangswerte zurück. Bei Kurogochi et al. (1985) zeigte sich der zirkadiane Verlauf der Corticosteron-Konzentration insoweit durch Nikotin verändert, dass die nächtlichen Werte höher lagen als bei Tieren, die kein Nikotin verabreicht bekamen. Seifert et al. 
Tabelle 3.31: Auswirkung einer chronischen Nikotingabe auf die HHN-Achse in tierexperimentellen Untersuchungen 1.

\begin{tabular}{|c|c|c|c|c|}
\hline \multirow[t]{2}{*}{ Autoren } & \multirow[t]{2}{*}{ Versuchstiere } & \multirow[t]{2}{*}{ Nikotindosis } & \multicolumn{2}{|c|}{ Nach Nikotingabe } \\
\hline & & & CS & АCTH \\
\hline Chen H et al. 2008 & Ratten & $\begin{array}{l}3 \mathrm{~d} \text { Nikotin (führten sich die } \\
\text { Ratten selbst zu) }\end{array}$ & $\uparrow(\operatorname{Tag} 1)$ & $\uparrow($ Tag 1, n. s.) \\
\hline Davis et al. 2005 & Ratten & 6 d 1 mg/kg Nikotin s. c. & $\uparrow$ & \\
\hline Caggiula et al.1991 & Ratten & $\begin{array}{l}10 \mathrm{~d} \text { 0,33, } 0,66 \mathrm{mg} / \mathrm{kg} \text { Nikotin } \\
\text { s. c. }\end{array}$ & $\uparrow$ & \\
\hline Pauly et al. 1992 & Mäuse & $12 \mathrm{~d} 6 \mathrm{mg} / \mathrm{kg}$ Nikotin i. p. & $\uparrow($ Tag 12$)$ & \\
\hline Morse 1989 & Kaninchen & $13 \mathrm{~d} 0,1-0,4 \mathrm{mg} / \mathrm{kg}$ Nikotin i. v. & $\uparrow($ n. s.) & \\
\hline Faraday et al. 2005 & $\begin{array}{l}\text { Ratten (Sprague- } \\
\text { Dawley/Long- } \\
\text { Evans) }\end{array}$ & $14 \mathrm{~d}$ 6, $12 \mathrm{mg} / \mathrm{kg}$ Nikotin s. c. & $\uparrow($ nur SD, w) & $\begin{array}{l}\uparrow(6 \mathrm{mg} / \mathrm{kg}, \\
\text { nur SD, w) }\end{array}$ \\
\hline Chen M et al. 2007 & Ratten (trächtig) & $14 \mathrm{~d} 2 \mathrm{mg} / \mathrm{kg}$ Nikotin s. c. & $\uparrow$ & \\
\hline
\end{tabular}

(1984) konnten keine Konzentrationsänderungen des Corticosterons während der 8 Wochen, in denen Ratten Nikotin gegeben wurde, feststellen. Cam und Bassett (1984) hingegen beobachteten in einem Zeitraum von 60 Tagen einen Anstieg der Hormone bis Tag 20 unter Nikotingabe.

Zusammenfassend zeigt sich auch nach einer chronischen Nikotingabe an Tiere ein recht einheitliches Bild. Die meisten Studien berichten über einen temporären Anstieg der CorticosteronKonzentration im Blut nach mehrtägiger Nikotingabe.

Weitere Aspekte Davis et al. (2005) und Caggiula et al. (1991) führten ihre Messungen auch

Tabelle 3.32: Auswirkung einer chronischen Nikotingabe auf die HHN-Achse in tierexperimentellen Untersuchungen 2.

\begin{tabular}{|c|c|c|c|}
\hline \multirow[t]{2}{*}{ Autoren } & \multirow[t]{2}{*}{ Versuchstiere } & \multirow[t]{2}{*}{ Nikotindosis } & $\begin{array}{l}\text { Nach } \\
\text { Nikotingabe }\end{array}$ \\
\hline & & & CS \\
\hline Benwell, Balfour 1979 & Ratten & $40 \mathrm{~d}$ 0,4 mg/kg Nikotin s. c. & $\uparrow($ bis Tag 5$)$ \\
\hline Kurogochi et al. 1985 & Ratten & 40 d 10 mg/kg Nikotin p. o. & $\uparrow($ nachts) \\
\hline Seifert et al. 1984 & Ratten & 8 Wochen 1 oder $2 \mathrm{mg} / \mathrm{kg}$ Nikotin s. c. & $\leftrightarrow$ \\
\hline Cam, Bassett 1984 & Ratten & $60 \mathrm{~d} 0,2 \mathrm{mg} / \mathrm{kg}$ Nikotin i. p. & $\uparrow($ bis Tag 20) \\
\hline
\end{tabular}


in anderen, den Tieren nicht bekannten Umgebungen durch, und es zeigte sich, dass die Tiere eine höhere Corticosteron-Konzentration im Blut aufwiesen, als ihre Vergleichsgruppe in gewohnter Umgebung. Ebenso hob ein Umgebungswechsel eine zuvor eingetretene Toleranz der Hormonkonzentration gegenüber Nikotin wieder auf.

Auch während einer längeren Nikotingabe wurde in einigen Studien mit Stresstests gearbeitet. Bei Faraday et al. (2005) wurde ein Teil der Versuchstiere täglich Stress ausgesetzt und Nikotin gegeben. Hierbei zeigte sich, dass die ACTH-Konzentration im Blut bei den weiblichen Ratten nach Stress und Nikotin höher war als nach Nikotingabe ohne Stresstest (je nach Rattenart bei 6 bzw. $12 \mathrm{mg} / \mathrm{kg} / \mathrm{d})$. Ähnliche Ergebnisse wurden bei einer Rattenart für die Corticosteron-Konzentration gefunden. Im Vergleich mit der Kontrollgruppe, die über 14 Tage Kochsalz erhalten hatte, wiesen die weiblichen Ratten nach 14-tägiger Nikotingabe im Stresstest jedoch geringere CorticosteronWerte auf. Morse (1989) beobachtete bei Kaninchen unter Nikotin einen nicht signifikanten Anstieg der Corticosteron-Werte. Wurden die Tiere Stress ausgesetzt und Nikotin gegeben, stieg die Hormonkonzentration signifikant höher an als mit Stress allein. Ähnliches konnten auch Chen $\mathrm{H}$ et al. (2008) beobachten. Ratten konnten sich über 20 Tage selbst Nikotin zuführen. An Tag 20 wurden die Hormone der HHN-Achse nach milden und moderaten Elektroschocks bestimmt. Die Konstellation milde Elektroschocks mit vorheriger Nikotingabe erzeugte höhere Hormonwerte als Elektroschocks und Kochsalz. Bei höheren Stromstärken hingegen unterschieden sich die Hormonkonzentrationen nicht zwischen Tieren, die Nikotin oder Kochsalz erhalten hatten. Wurden die Tiere Immobilisationsstress ausgesetzt, unterschieden sich die Corticosteron- und ACTH-Werte nicht zwischen Nikotin- und Kontrollgruppe.

\section{Nikotinentzug}

Vier der 37 Studien haben sich mit einem der Nikotingabe folgenden Nikotinentzug bei Tieren auseinandergesetzt. Tabelle 3.33 führt diese auf.

Benwell und Balfour (1979) spritzten Ratten für 39 Tage Nikotin und bestimmten währenddessen und an Tag 40 die Corticosteron-Konzentration im Blut der Tiere. Bis Tag fünf war die Hormonkonzentration erhöht (s. o.) und lag bis Tag 39 wieder auf Ausgangsniveau. Die fehlende Nikotininjektion an Tag 40 ließ die Corticosteron-Konzentration wieder steigen. Semba et al. 
(2004) konnten zwei Tage nach einer zwölftägigen Nikotingabe an Ratten keine Hormonkonzentrationsunterschiede zwischen Ratten, die Nikotin oder Kochsalz erhalten hatten, ausmachen. Nach einem Stresstest hingegen lagen die Corticosteron-Werte der Ratten, die vor den zwei injektionsfreien Tagen Nikotin erhalten hatten, deutlich unter den Werten der Kontrollgruppe. Andersson et al. (1989) setzten Ratten für zehn Tage Zigarettenrauch aus und bestimmten zwei, drei und sieben Tage später die Hormone der HHN-Achse und verglichen diese Werte mit denen einer Kontrollgruppe, die Kochsalz erhalten hatte. Bis auf ein Absinken der Corticosteron-Konzentration am zweiten Entzugstag konnten sie keine Unterschiede feststellen. 15 Tage nach einer zwölftägigen Nikotingabe an Mäuse konnten Pauly et al. (1992) erhöhte Corticosteron-Werte im Blut im Vergleich mit der Kontrollgruppe beobachten. Nach einer Nikotingabe am zweiten Entzugstag fiel die Corticosteron-Konzentration ab.

Zusammengenommen liefern die Studien kein einheitliches Bild. Betrachtet man beispielsweise die Entzugszeit von zwei Tagen, widersprechen sich die Veröffentlichungen von Semba et al. (2004), Andersson et al. (1989) und Pauly et al. (1992).

\section{Zusammenfassung}

Tabelle 3.34 fasst die Ergebnisse der Studien zusammen, die eine Nikotingabe in tierexperimentellen Untersuchungen beobachtet haben.

Tabelle 3.33: Auswirkung eines Nikotinentzugs auf die HHN-Achse in tierexperimentellen Untersuchungen.

\begin{tabular}{llllll}
\hline Autoren & Versuchstiere & Nikotindosis & Entzugsdauer & Während des Entzugs \\
\cline { 4 - 6 } & & & CS & ACTH \\
\hline Benwell, Balfour 1979 & Ratten & $40 \mathrm{~d} 0,4 \mathrm{mg} / \mathrm{kg}$ Nikotin s. c. & $1 \mathrm{~d}$ & $\uparrow$ \\
Semba et al. 2004 & Ratten & $14 \mathrm{~d} 6 \mathrm{mg} / \mathrm{kg}$ Nikotin s.c. & $2 \mathrm{~d}$ & $\leftrightarrow$ \\
Andersson et al. 1989 & Ratten & $10 \mathrm{~d}$ & $7 \mathrm{~d}$ & $\downarrow$ (an Tag 2) & $\leftrightarrow$ \\
& & Zigarettenrauch-Exposition & & \\
Pauly et al. 1992 & Mäuse & $12 \mathrm{~d}$ 6 mg/kg Nikotin i.p. & $15 \mathrm{~d}$ & $\uparrow$ \\
\hline
\end{tabular}


Tabelle 3.34: Auswirkungen einer Nikotingabe auf die HHN-Achse in tierexperimentellen Untersuchungen (Zusammenfassung).

\begin{tabular}{llll}
\hline & Studienanzahl & Einheitlichkeit $^{a}$ & $\begin{array}{l}\text { Ergebnisse: } \\
\text { HHN- } \\
\text { Hormone }\end{array}$ \\
\hline Akute Nikotingabe & 24 & ja & $\uparrow$ \\
Chronische Nikotingabe & 11 & ja & $\uparrow$ \\
Entzug & 4 & nein & $\uparrow \leftrightarrow \downarrow$ \\
${ }^{a}$ Mindestens 70\% der Studien haben ähnliche Ergebnisse. &
\end{tabular}

\subsubsection{Nikotingabe an Nichtraucher}

Die Suche nach Studien, in denen Hormone der HHN-Achse nach Nikotingabe an Nichtraucher bestimmt wurden, lieferte 336 Suchergebnisse, von denen sechs Studien den Suchkriterien entsprechen. Man stößt auf vier Forschungsgruppen, die in der Vergangenheit dieser Fragestellung nachgegangen sind. Es gibt eine aktuelle Veröffentlichung aus dem Jahr 2008, die übrigen sind bis 1990 erschienen.

In vier Studien wurde die Nikotinwirkungen bei Rauchern und Nichtrauchern miteinander verglichen, eine Studie beschäftigte sich neben gesunden Probanden auch mit Probanden, die an M. Alzheimer erkrankt waren und in einer letzten Studie wurde neben Nikotin ein Placebo eingesetzt.

Tabelle 3.35 bietet eine Zusammenfassung der Studienergebnisse, auf die nun näher eingegangen wird.

Gossain et al. (1986) verglichen die Blut-Cortisolwerte von Nichtrauchern und Rauchern vor und nach dem Konsum von zwei Zigaretten. Es zeigte sich, dass beide Probandengruppen vor dem Rauchen annähernd gleiche Cortisolspiegel hatten, nach dem Zigarettenkonsum jedoch nur bei Rauchern eine erhöhte Cortisolkonzentration im Blut festgestellt wurde. Dieses Ergebnis steht im Widerspruch zu den anderen Studien, die in Tabelle 3.35 aufgeführt sind. So fanden Sellini et al. (1989a,b,c) in ihren Veröffentlichungen eine Erhöhung der Cortisolspiegel im Plasma bei nikotinnaiven Menschen wenige Minuten nach Nikotinapplikation - bei Rauchern hingegen nicht. Sie wählten zwei unterschiedliche Arten der Verabreichung: Die passive Inhalation und die orale 
Aufnahme mittels Nikotinkaugummi (2 mg Nikotin). Außerdem bestimmten Sellini et al. (1989a,b,c) zusätzlich das ACTH im Plasma und auch dieser Wert stieg nur bei Nichtrauchern nach Nikotingabe an. Interessant ist, dass sich eine Erhöhung der Hormone nach Inhalation von Zigarettenrauch nur bei solchen Probanden zeigte, welche für gewöhnlich keinem Passivrauch ausgesetzt waren. Newhouse et al. (1990) infundierten elf Nichtrauchern 0,25 oder $0,5 \mu \mathrm{g} / \mathrm{kg} / \mathrm{min}$ Nikotin oder ein Placebo über 60 Minuten und verglichen die Cortisol- und ACTH-Spiegel aus dem Blut mit denen von elf Nichtrauchern, die an der Alzheimer-Krankheit litten. Dieser Probandengruppe wurde auch Nikotin $(0,125,0,25$ oder $0,5 \mu \mathrm{g} / \mathrm{kg} / \mathrm{min})$ oder ein Placebo über 60 Minuten infundiert. Bei beiden Gruppen stieg der Cortisol- und ACTH-Spiegel nach intravenösem Nikotin dosisabhängig an und fiel nach circa drei Stunden auf den Ausgangswert ab. Zudem wurde eine Korrelation zwischen dem Anstieg dieser Parameter und einer vermehrten Unruhe der Probanden gefunden. Perkins et al. (2008) verabreichten 101 Nichtrauchern Nikotin über ein Nasenspray. Innerhalb von 30 Minuten wurde den Probanden achtmal eine bestimmte Dosis (5 oder $10 \mu \mathrm{g} / \mathrm{kg}$ ) verabreicht und dieses Prozedere dreimal wiederholt. Diese Studie zeigte einen Anstieg der Cortisolkonzentration im Speichel nach Nikotinapplikation (10 $\mu \mathrm{g} / \mathrm{kg}$ Nikotin) - allerdings nur bei Probanden, die das 7-Repeat-Allel des D4-Rezeptors trugen.

Zusammenfassend wurden in fünf von sechs Studien nach Nikotingabe an Nichtraucher erhöhte Cortisol- und ACTH-Werte gefunden.

\section{Zusammenfassung}

Tabelle 3.36 fasst die Ergebnisse der Studien, die eine Nikotingabe an Nichtraucher untersuchten, zusammen.

\subsubsection{Nikotinentzug bei Gelegenheitsrauchern}

Die Suche erbrachte mit den verwendeten Stichwörtern vier Suchergebnisse. Keine dieser Studien entspricht den Suchkriterien. 
Tabelle 3.35: Auswirkungen einer Nikotingabe auf die HHN-Achse bei Nichtrauchern.

\begin{tabular}{|c|c|c|c|c|}
\hline \multirow[t]{2}{*}{ Autoren } & \multirow[t]{2}{*}{ Studiendesign } & \multirow[t]{2}{*}{ Nikotindosis } & \multicolumn{2}{|c|}{ Nach Nikotingabe } \\
\hline & & & Cortisol & АCTH \\
\hline Gossain et al. 1986 & Nicht kontrolliert & 2 Zigaretten & $\leftrightarrow$ & \\
\hline Sellini et al. 1989a & Nicht kontrolliert & Zigarettenrauch-Exposition & $\uparrow$ & $\uparrow$ \\
\hline Sellini et al. 1989b & Nicht kontrolliert & Zigarettenrauch-Exposition & $\uparrow$ & $\uparrow$ \\
\hline Sellini et al. 1989c & Nicht kontrolliert & $\begin{array}{l}\text { Nikotinkaugummis ( } 2 \mathrm{mg} \\
\text { Nikotin) }\end{array}$ & $\uparrow$ & $\uparrow$ \\
\hline Newhouse et al. 1990 & $\begin{array}{l}\text { Einfachblind, } \\
\text { placebokontrolliert }\end{array}$ & $\begin{array}{l}0,25,0,5 \mu \mathrm{g} / \mathrm{kg} / \mathrm{min} \text { Nikotin i. v. } \\
\text { über } 60 \mathrm{~min}\end{array}$ & $\uparrow$ & $\uparrow$ \\
\hline Perkins et al. 2008 & $\begin{array}{l}\text { Einfachblind, } \\
\text { placebokontrolliert }\end{array}$ & $\begin{array}{l}8 \text { mal Nikotinnasenspray ( } 5 \\
\text { oder } 10 \mu \mathrm{g} / \mathrm{kg} \text { Nikotin) }\end{array}$ & $\begin{array}{l}\uparrow \\
(10 \mu \mathrm{g} / \mathrm{kg})^{a}\end{array}$ & \\
\hline
\end{tabular}

${ }^{a}$ Bei Trägern des 7-Repeat-Allels des D4-Rezeptors.

Tabelle 3.36: Auswirkungen einer Nikotingabe auf die HHN-Achse bei Nichtrauchern (Zusammenfassung).

\begin{tabular}{|c|c|c|c|}
\hline Art der Nikotingabe & Studienanzahl & Einheitlichkeit $^{a}$ & $\begin{array}{l}\text { Ergebnisse: } \\
\text { HHN-Hormone }\end{array}$ \\
\hline Aktives Rauchen & 1 & & $\leftrightarrow$ \\
\hline Passives Rauchen & 2 & ja & $\uparrow$ \\
\hline Kaugummi & 1 & & $\uparrow$ \\
\hline Infusion & 1 & & $\uparrow$ \\
\hline Nasenspray & 1 & & $\uparrow^{b}$ \\
\hline
\end{tabular}

${ }^{a}$ Mindestens $70 \%$ der Studien haben ähnliche Ergebnisse. ${ }^{b}$ Genetische Untergrupppe. 


\subsubsection{Nikotinentzug bei Rauchern}

Die Suche zu diesem Themengebiet erbrachte 512 Suchergebnisse, von denen 20 den Suchkriterien entsprechen. Zwölf Forschungsgruppen haben sich mit der Frage beschäftigt, welche hormonellen Auswirkungen ein Nikotinentzug bei Rauchern hat. Die frühste Veröffentlichung ist aus dem Jahr 1982, die aktuellste von 2011.

Die Forschungsgruppen von O. und C. Pomerleau, M. al'Absi, M. Ussher und D. Gilbert haben mehr als eine Veröffentlichung zu diesem Themengebiet beitragen. Eingeteilt und besprochen werden die Studien im Folgenden nach der Länge des Nikotinentzugs.

\section{Auswirkungen eines mehrstündigen Nikotinentzugs}

Acht der 20 Studien untersuchten einen mehrstündigen Nikotinentzug bei Rauchern. Sie entstammen aus den sechs Forschungsgruppen um D. Cherek, O. und C. Pomerleau, J. Hughes, M. Ussher, N. Benowitz und M. al'Absi, der drei Veröffentlichungen zu diesem Abschnitt beiträgt. Eine Übersicht bietet Tabelle 3.37.

Cherek et al. (1982) maßen während mehrerer zweistündiger Sitzungen die Cortisolkonzentration der Probanden im Blut und Speichel. In der Hälfte dieser Sitzungen hatten die Teilnehmer eine einfache Aufgabe zu bearbeiten, in der anderen nicht. Während dieser beiden Sitzungsarten mussten die vier männlichen Raucher entweder Zigaretten konsumieren oder abstinent bleiben. Cherek et al. (1982) fanden keine Konzentrationsunterschiede des Cortisols zwischen den einzelnen Bedingungen. Diese Beobachtung machten auch al'Absi et al. (2002) in einer ihrer Studien. Sie konnten keine Unterschiede in der Cortisolkonzentration im Speichel zwischen Probanden, die geraucht hatten oder 18 Stunden abstinent geblieben waren, ausmachen. In ihrer anderen Studie in diesem Abschnitt (Cohen et al., 2004) beobachteten die Autoren um M. al'Absi hingegen nach vierstündiger Abstinenz 20 männlicher Raucher einen Abfall in der Speichel-Cortisolkonzentration. Zudem entdeckten sie einen Zusammenhang: Je höher die Cortisolkonzentration vor dem Entzug war, desto stärker waren die Entzugserscheinungen im Entzug. Pomerleau et al. (1992) fanden nach einer zwölfstündigen Abstinenz acht weiblicher Teilnehmerinnen neben einer positiven Korrelation von Entzugs- und Menstruationssymptomen eine geringere Cortisolkonzentration 
im Blut der Probandinnen als unter Nikotinkonsum. Ussher et al. (2011) konnten nach einer 15-stündigen Abstinenz keine Veränderungen in der Speichel-Cortisolkonzentration ausmachen. Ebenso wurde keine Korrelation zwischen Hormonkonzentration und subjektiven Parametern beobachtet. Die Gabe von Hydrocortison hob die Cortisolspiegel nach einer Dosis von $40 \mathrm{mg}$ an, das Verlangen nach Zigaretten oder Entzugserscheinungen wurden dadurch allerdings nicht beeinflusst. Auch Benowitz et al. (1984) stellten keine Unterschiede in der Cortisolkonzentration nach einer Entzugszeit von 24 Stunden fest. Al'Absi et al. (2004) hingegen beobachteten bei den nach einer Woche rückfälligen Probanden einen stärkeren Abfall der Speichel-Cortisolkonzentration als bei Probanden, die nach einer Woche noch abstinent waren. In der letzten Studie aus Tabelle 3.37 (Hughes et al., 1988) wurde nach einer 24-stündigen Abstinenz der zehn Probanden eine höhere Blut-Cortisolkonzentration beobachtet als unter der Bedingung, in der geraucht wurde. Eine Korrelation zwischen Cortisolanstieg und Entzugserscheinungen wurde nicht gefunden. Der ebenfalls durchgeführte Dexamethason-Suppressions-Test (DST) lieferte im Entzug dieselben Ergebnisse wie unter Nikotin.

Zusammenfassend ergibt sich beim Betrachten der Studienergebnisse kein einheitliches Bild. Unter Berücksichtigung, dass wenige der acht Studien dieselbe Entzugszeit untersuchte, kann folgendes festgehalten werden: Drei Studien beobachteten einen Abfall, eine einen Anstieg der Cortisolkonzentration. Vier weitere Veröffentlichungen konnten keine Änderungen der Cortisolwerte im Entzug feststellen.

\section{Auswirkungen eines mehrtägigen Nikotinentzugs}

Tabelle 3.38 listet die sieben Studien auf, die sich mit einem mehrtägigen Nikotinentzug bei Rauchern beschäftigten. Fünf Forschungsgruppen haben dies untersucht, im einzelnen die von W. Pickworth, V. Teneggi, A. Rasmusson, M. al'Absi und O. und C. Pomerleau. Die letzten beiden Forschungsgruppen veröffentlichten jeweils zwei Studien zu diesem Thema.

Ceballos und al'Absi (2006) führten bei 68 Rauchern einen zweitägigen Entzug durch und konnten keine Veränderungen der Speichel-Cortisolkonzentration erfassen. Auch gab es keine Unterschiede in den Cortisolspiegeln der zwei Beobachtungstage zwischen Probanden, die nach vier Wochen 
Tabelle 3.37: Auswirkung eines mehrstündigen Nikotinentzugs auf die HHNAchse bei Rauchern.

\begin{tabular}{|c|c|c|c|}
\hline \multirow[t]{2}{*}{ Autoren } & \multirow[t]{2}{*}{ Studiendesign } & \multirow[t]{2}{*}{ Entzugsdauer } & \multirow{2}{*}{$\begin{array}{l}\text { Während des } \\
\text { Entzugs } \\
\text { Cortisol }\end{array}$} \\
\hline & & & \\
\hline Cherek et al. 1982 & Randomisiert, kontrolliert & $2 \mathrm{~h}$ & $\leftrightarrow$ \\
\hline Cohen et al. 2004 & Nicht kontrolliert & $4 \mathrm{~h}$ & $\downarrow$ \\
\hline Pomerleau et al. 1992 & Nicht kontrolliert & $12 \mathrm{~h}$ & $\downarrow$ \\
\hline Ussher et al. 2011 & $\begin{array}{l}\text { Doppelblind, randomisiert, } \\
\text { placebokontrolliert, } \\
\text { Cross-over }\end{array}$ & $15 \mathrm{~h}$ & $\leftrightarrow$ \\
\hline al'Absi et al. 2002 & Nicht kontrolliert & $18 \mathrm{~h}$ & $\leftrightarrow$ \\
\hline al'Absi et al. 2004 & Nicht kontrolliert & $24 \mathrm{~h}$ & $\downarrow$ \\
\hline Benowitz et al. 1984 & Nicht kontrolliert & $24 \mathrm{~h}$ & $\leftrightarrow$ \\
\hline Hughes et al. 1988 & Nicht kontrolliert & $24 \mathrm{~h}$ & $\uparrow$ \\
\hline
\end{tabular}

noch abstinent (17 Probanden) oder rückfällig (51 Probanden) geworden waren. In dieser Studie wurde, wie auch bei Rasmusson et al. (2006), die Dehydroepiandrosteronsulfat- (DHEAS-) Konzentration gemessen und in Verhältnis zu Cortisol gesetzt. Der Quotient Cortisol/DHEAS unterschied sich nicht zwischen Rückfälligen und Abstinenten. Ebenso konnte keine Korrelation der Hormonkonzentrationen mit einer Rückfallwahrscheinlichkeit gefunden werden. Auch Pickworth et al. (1996) konnten während eines dreitägigen Nikotinentzugs, in dem Placebopflaster verwendet wurden, keine Änderungen der Cortisol- oder ACTH-Konzentrationen im Blut nachweisen. Wenn jedoch Nikotinpflaster (in der Dosierung 10, 20 oder $30 \mathrm{mg}$ Nikotin über 16 Stunden) eingesetzt wurden, konnte ein Anstieg der Cortisol-, wie auch der ACTH-Konzentration am zweiten Entzugstag beobachtet werden. Teneggi et al. (2002), die auch Nikotin- und Placebopflaster während eines dreitägigen Entzugs verwendeten, konnten die Ergebnisse von Pickworth et al. (1996) nicht voll bestätigen. Sie beobachteten zwar auch keine Unterschiede in der Blut-Cortisolkonzentration im Entzug unter Verwendung von Placebopflastern, einen Anstieg der Cortisolkonzentration unter Nikotinpflastern (21 mg Nikotin über 24 Stunden) fanden sie hingegen nicht. In dieser Studie wurde das Cortisol auch im Urin bestimmt, und es zeigte sich am ersten Entzugstag eine geringere Cortisolkonzentration bei den Probanden, die kein Nikotin appliziert bekommen hatten. In einer weiteren Studie (Kotlyar et al., 2006) konnte ebenfalls keine Änderung der Cortisolkonzen- 
tration im Blut während eines dreitägigen Nikotinentzugs beobachtet werden. Dieses Ergebnis entstand unter Verwendung eines Bupropion-Placebos, welches 17 Tage lang vor dem Entzug eingenommen wurde. In dieser Arbeit wurde z. T. unter Stressbedingungen gemessen - einmal unter Nikotinkonsum und einmal nach dreitägiger Abstinenz. In der Abstinenz wurden nur nach 17-tägiger Bupropiongabe (300 mg) erhöhte Cortisolspiegel während der Stresstests beobachtet. Einen viertägigen Entzug untersuchten Pomerleau et al. (2004). Als Probanden dienten Raucherinnen; Die Hälfte der Frauen litt zudem unter Depressionen. Auch hier kam es im Entzug zu keiner Konzentrationsänderung des Cortisols oder ACTHs im Blut, unabhängig davon, ob Depressionen vorlagen oder nicht. Unterschiede gab es allerdings bei den Entzugserscheinungen, die vermehrt bei den depressiven Frauen auftraten. Das ACTH wurde außerdem während des Entzugs bei den depressiven Probandinnen im DST kaum supprimiert. Auch Rasmusson et al. (2006) beschäftigten sich mit depressiven Symptomen und erfassten diese während eines Entzugs von acht Tagen. Bei den Probanden wurden die Cortisol-, Dehydroepiandrosteron- (DHEA-) und DHEAS-Spiegel im Blut gemessen und am 15. Tag der Raucherstatus bestimmt. Eine signifikante Veränderung der Cortisolkonzentration wurde nicht beobachtet, aber ein Trend für einen Cortisolanstieg bei den Probanden, die einen Rückfall erlitten. Bei den am 15. Tag noch abstinent Gebliebenen wurde ein Trend für einen Cortisolabfall registriert. Auch war bei dieser abstinenten Gruppe das Verhältnis von DHEA zu Cortisol erhöht, bei den Rückfälligen erniedrigt. Nur bei Frauen wurde eine positive Korrelation von Entzugserscheinungen und depressiven Symptomen mit dem Cortisolspiegel beobachtet (außerdem eine negative Korrelation von Entzugserscheinungen und depressiven Symptomen mit dem Verhältnis von DHEA(S) zu Cortisol). Die letzte Publikation in der Tabelle 3.38 ist eine weitere von Pomerleau et al. (2000). Hier sank die Cortisolkonzentration während eines elftägigen Entzugs ab.

Betrachtet man zusammenfassend die Studienergebnisse, die ohne medikamentöse Interventionen erhalten wurden, wurde in sechs von sieben Studien eine unveränderte Cortisolkonzentration im Blut bzw. Speichel während eines Nikotinentzugs beobachtet. In einer Studie wurde ein vorübergehender Cortisolabfall im Urin festgestellt. Lediglich eine weitere Studie fand einen Abfall des Cortisolspiegels im Entzug. 
Tabelle 3.38: Auswirkung eines mehrtägigen Nikotinentzugs auf die HHN-Achse bei Rauchern.

\begin{tabular}{|c|c|c|c|c|}
\hline \multirow[t]{2}{*}{ Autoren } & \multirow[t]{2}{*}{ Studiendesign } & \multirow[t]{2}{*}{ Entzugsdauer } & \multicolumn{2}{|c|}{ Während des Entzugs } \\
\hline & & & Cortisol & АСТН \\
\hline $\begin{array}{l}\text { Ceballos, al'Absi } \\
2006\end{array}$ & Nicht kontrolliert & $2 \mathrm{~d}$ & $\leftrightarrow$ & \\
\hline Kotlyar et al. 2006 & $\begin{array}{l}\text { Einfachblind, randomisiert, } \\
\text { placebokontrolliert }\end{array}$ & $3 \mathrm{~d}$ & $\leftrightarrow$ & \\
\hline Pickworth et al. 1996 & $\begin{array}{l}\text { Doppelblind, randomisiert, } \\
\text { placebokontrolliert, } \\
\text { Cross-over }\end{array}$ & $3 \mathrm{~d}$ & $\leftrightarrow$ & $\leftrightarrow$ \\
\hline Teneggi et al. 2002 & $\begin{array}{l}\text { Doppelblind, randomisiert, } \\
\text { placebokontrolliert, } \\
\text { Cross-over }\end{array}$ & $3 \mathrm{~d}$ & $\begin{array}{l}\text { Blut: } \leftrightarrow \text {, Urin: } \downarrow \\
\text { (Tag 1) }\end{array}$ & \\
\hline Pomerleau et al. 2004 & Nicht kontrolliert & $4 \mathrm{~d}$ & $\leftrightarrow$ & $\leftrightarrow$ \\
\hline $\begin{array}{l}\text { Rasmusson et al. } \\
2006\end{array}$ & Nicht kontrolliert & $8 \mathrm{~d}$ & $\leftrightarrow$ & \\
\hline Pomerleau et al. 2000 & Nicht kontrolliert & $11 \mathrm{~d}$ & $\downarrow$ & \\
\hline
\end{tabular}

\section{Auswirkungen eines mehrwöchigen Nikotinentzugs}

Vier Forschungsgruppen beschäftigten sich mit einem mehrwöchigen Nikotinentzug. Tabelle 3.39 bietet eine Übersicht über die Studien der Forschungsgruppen von D. Gilbert (die zwei Studien veröffentlichten), S. Frederick, I. Puddey und M. Ussher.

Drei Veröffentlichungen (Meliska et al., 1995; Frederick et al., 1998; Gilbert et al., 1999) erfassten während eines vierwöchigen (bzw. eines 31-tägigen) Entzugs die Cortisolspiegel der Probanden. Die Forschungsgruppe um D. Gilbert beobachtete in ihren beiden Studien (Meliska et al., 1995; Gilbert et al., 1999) ein signifikantes Absinken der Serum-Cortisolkonzentration im Entzug. Auch bei Frederick et al. (1998) wurde in der zweiten und vierten Entzugswoche ein Abfall der SpeichelCortisolkonzentration registriert, nicht jedoch ein verändertes Ergebnis des durchgeführten DSTs. In dieser Studie wurde 52 Wochen nach Beginn der Abstinenz der Raucherstatus überprüft und festgestellt, dass ein stärkerer Abfall des Cortisolspiegels mit vermehrten Entzugserscheinungen korreliert war und einen Rückfall nicht signifikant wahrscheinlicher machte als ein nicht so starker Abfall der Hormonkonzentration. In dieser Studie wurden die Messergebnisse der Männer und Frauen miteinander verglichen und man kam zu dem Ergebnis, dass es hierbei zwischen den 
Geschlechtern keine Unterschiede gab. Puddey et al. (1984) und Ussher et al. (2006) führten einen Entzug von sechs Wochen durch und kamen ebenfalls zu dem Resultat, dass die Cortisolkonzentration in dieser Zeit abfällt. In der Arbeit von Ussher et al. (2006) trugen die Probanden z. T. Nikotinpflaster (15 mg) über 16 Stunden, aber auch bei diesen Teilnehmern zeigte sich der Cortisolabfall. Bei den 30 von 112 Rauchern, die abstinent blieben, konnte nach einem starken anfänglichen Abfall des Cortisolspiegels eine langsame Zunahme der Hormonkonzentration im Verlauf der Entzugswochen beobachtet werden (die Cortisolspiegel blieben aber signifikant erniedrigt). Ein Unterschied zwischen Probanden mit und ohne Nikotinpflaster wurde nicht beobachtet. Bei Männern und Frauen zeigten sich ähnliche Ergebnisse. Ussher et al. (2006) fiel zudem eine nicht signifikante positive Korrelation zwischen Cortisolabfall und Rückfallwahrscheinlichkeit auf sowie eine negative Korrelation zwischen Entzugserscheinungen und der absoluten Cortisolkonzentration im Entzug.

Tabelle 3.39: Auswirkung eines mehrwöchigen Nikotinentzugs auf die HHNAchse bei Rauchern.

\begin{tabular}{llll}
\hline Autoren & Studiendesign & Entzugsdauer & $\begin{array}{l}\text { Während des } \\
\text { Entzugs }\end{array}$ \\
\cline { 4 - 4 } & & & Cortisol \\
\hline Frederick et al. 1998 & Nicht kontrolliert & 4 Wochen & $\downarrow$ \\
Gilbert et al. 1999 & Randomisiert, kontrolliert & 31 Tage & $\downarrow$ \\
Meliska et al. 1995 & Randomisiert, kontrolliert & 31 Tage & $\downarrow$ \\
Puddey et al. 1984 & Randomisiert, kontrolliert & 6Wochen & $\downarrow$ \\
Ussher et al. 2006 & Nicht kontrolliert & 6 Wochen & $\downarrow$ \\
\hline
\end{tabular}

Zusammenfassend berichten alle Studien, in denen ein mehrwöchiger Entzug von Rauchern untersucht wurde, dass die Cortisolkonzentration in dieser Zeit abfällt.

\section{Zusammenfassung}

Tabelle 3.40 fasst die Ergebnisse der Studien, die einen Entzug von Rauchern untersuchten, zusammen. 
Tabelle 3.40: Auswirkungen eines Nikotinentzugs auf die HHN-Achse bei Rauchern (Zusammenfassung).

\begin{tabular}{llll}
\hline $\begin{array}{l}\text { Dauer des } \\
\text { Entzugs }\end{array}$ & Studienanzahl & Einheitlichkeit & \\
& & $\begin{array}{l}\text { Ergebnisse: } \\
\text { HHN-Hormone }\end{array}$ \\
\hline Stunden & 8 & nein & $\uparrow \leftrightarrow \downarrow$ \\
Tage & 7 & ja & $\leftrightarrow$ \\
Wochen & 5 & ja & $\downarrow$ \\
\hline
\end{tabular}

${ }^{a}$ Mindestens $70 \%$ der Studien haben ähnliche Ergebnisse. 


\section{Diskussion}

Nach aktuellem Kenntnisstand ist dies die erste systematische Übersichtsarbeit, die sich durch eine umfassende Literaturrecherche mit den Auswirkungen von Nikotin auf objektive Schlafparameter, den Neurotransmitter Dopamin und auf die Hypothalamus-Hypophysen-Nebennieren-Achse beschäftigt. Hierbei sind neben Veröffentlichungen, die Nichtraucher, Gelegenheitsraucher und Raucher untersuchten, auch tierexperimentelle Studien von Interesse. Insbesondere hat diese Übersichtsarbeit einen Fokus auf den Nikotinentzug, um mögliche Prädiktoren für einen Rückfall zu identifizieren.

Durch die Literaturrecherche wurden 3037 Veröffentlichungen ausfindig gemacht, von denen 93 den Suchkriterien entsprechen und in den Ergebnisteil aufgenommen worden sind. Ein Großteil der Ergebnisse beschäftigt sich mit der HHN-Achse. Die Mehrzahl der Veröffentlichungen besteht aus nicht kontrollierte Studien.

Es konnten keine Veröffentlichungen gefunden werden, die sich mit Gelegenheitsrauchern beschäftigten oder die Konsequenzen einer Nikotingabe auf das dopaminerge System bei Nichtrauchern untersuchten. Zudem konnten keine Studien ausfindig gemacht werden, in denen Nichtraucher nach einer Nikotingabe mit Rauchern oder Gelegenheitsrauchern bezüglich des Schlafs oder dopaminerger Parameter verglichen wurden.

Zusammenfassend fällt auf, dass sich die Wirkungen von Nikotin oder eines Nikotinentzugs auf den Schlaf oder die HHN-Achse bei den untersuchten Gruppen ähneln. So stieg die Cortisolbzw. Corticosteroid- und ACTH-Konzentration nach einer Nikotingabe an Nichtraucher und in tierexperimentellen Untersuchungen an. Hierbei scheint die Art und Weise der Applikation keine Rolle zu spielen, fast alle Studien beobachteten einen Anstieg der Hormone der HHN-Achse. Des Weiteren konnte in den tierexperimentellen Studien, die sich mit polysomnografischen Parametern 
beschäftigten, festgestellt werden, dass höhere Nikotindosen die Wachheit förderten (verbunden mit einer Abnahme des REM- und NREM-Schlafs) - unabhängig von der Dauer der Nikotingabe. Auch in den Studien, die Nichtraucher untersuchten, konnte zumindest eine Abnahme des REMSchlafs beobachtet werden.

Betrachtet man die Studien, die einen Entzug von wenigen Tagen polysomnografisch untersucht haben, fällt eine weitere Gemeinsamkeit auf: Weder für gesunde oder depressive Nichtraucher, die nach einer Nikotingabe am Absetztag untersucht wurden, noch für Raucher im Entzug änderte sich die Gesamtschlafzeit oder die REM- oder NREM-Schlafzeit.

Uneinheitliche Ergebnisse wurden hingegen für die Hormone der HHN-Achse im Entzug von wenigen Stunden bis Tagen gefunden. In den tierexperimentellen Studien und in den Veröffentlichungen, in denen ein Entzug von Rauchern untersucht wurde, konnten keine einheitlichen Ergebnisse beobachtet werden.

In Studien, in denen eine Nikotingabe oder ein Nikotinentzug aktigrafisch untersucht wurde, konnten weder für Nichtraucher nach Nikotingabe noch für Raucher im Entzug Änderungen der Aktivität im Schlaf gemessen werden.

Zusammenfassend kann festgehalten werden, dass es wenige Studien zu einem Großteil der genannten Themengebiete gibt. Beispielsweise ergab die Suche nach Veröffentlichungen, die sich mit dem dopaminergen System beschäftigten, nur fünf Studien. Ähnlich sieht es für Studien aus, die Nichtraucher nach einer Nikotingabe untersuchten. Diesbezüglich fiel zudem auf, dass nur wenige Forschungsgruppen an der Veröffentlichung der gefundenen Studien beteiligt waren.

Bei der Literaturrecherche wurden zwei Veröffentlichungen gefunden, die eine ähnliche systematische Übersicht über die Wirkung von Nikotin und eines Nikotinentzugs bezüglich des Schlafs und der HHN-Achse bieten. Jähne et al. (2010) führten eine systematische Literaturrecherche durch, beschränkten sich aber auf die Folgen einer Nikotingabe und eines Nikotinentzugs auf den Schlaf. Alle Studien, die von Jähne et al. (2010) gefunden wurden, sind - wenn sie den Suchkriterien entsprechen - auch Teil der vorliegenden Übersichtsarbeit, die zusätzlich aktuelle Studien bis 2012 und zwei weitere Themengebiete (dopaminerges System und HHN-Achse) beinhaltet. Berlin 
(2009) veröffentlichte eine Übersichtsarbeit zu den endokrinen Konsequenzen eines Nikotinentzugs. Hierbei konzentrierte sich der Autor auf den Zeitraum August 1998 bis Juli 2008 und auf Humanstudien, die in englischer Sprache verfasst worden sind. Für die vorliegende Arbeit konnten in derselben Zeitspanne acht weitere Studien ausfindig gemacht werden. Zudem weist die vorliegende Arbeit alle Studien auf, die auch bei Berlin (2009) zu finden sind, wenn sie den Suchkriterien entsprechen.

\subsection{Allgemeine Anmerkungen zu den Ergebnissen}

Bei der beurteilenden Betrachtung der Ergebnisse müssen einige grundlegende Dinge beachtet werden. Eine Vergleichbarkeit der Studienresultate ist meist nicht ohne Einschränkungen gegeben, zu unterschiedlich sind die Fragestellungen und Methoden der einzelnen Studien.

Probanden und Versuchstiere Schaut man sich die menschlichen Probandengruppen an, variieren Alter, Geschlechterverteilung, ethnische Gruppe, Vorerkrankungen, Medikamenteneinnahme und bei den Rauchern Dauer und Stärke der Nikotinabhängigkeit, um einige wichtige Differenzierungsmerkmale zu benennen. Ein Vergleich dieser Probandengruppen miteinander ist insofern problematisch, da diese möglicherweise völlig unterschiedlich auf die Studieninterventionen reagieren. Beispielsweise könnte ein mitteleuropäischer, gesunder, stark rauchender Mann einen anderen Hormonkonzentrationsverlauf im Nikotinentzug aufweisen als eine ältere asiatische Frau, die weniger stark raucht und schon eine längere Krankengeschichte hat. Im Abschnitt über die Konsequenzen eines Nikotinentzugs auf die Hormone der HHN-Achse lag z. B. das Durchschnittsalter der Probanden bei Gilbert et al. (1999) bei circa 28 Jahren, bei Ussher et al. (2006) bei circa 43 Jahren. Cherek et al. (1982) und Cohen et al. (2004) untersuchten nur männliche Probanden, Pomerleau et al. (2004) nur weibliche usw.

In den tierexperimentellen Arbeiten wurden unterschiedliche Tierarten untersucht, zumeist Ratten, Mäuse oder Katzen. Ein Vergleich von verschiedenen Tierordnungen miteinander muss ebenso kritisch betrachtet werden wie Rückschlüsse von tierexperimentell gewonnenen Ergebnissen auf den Menschen. 
Fragestellung Weitere Gründe für die eingeschränkte Vergleichbarkeit sind die unterschiedlichen Fragestellungen, Abläufe und Interventionen der Studien. Ein Teil der dargestellten Ergebnisse sind Studien entnommen, deren primäres Studienziel nicht der Erhalt dieser Ergebnisse war. Sie sind vielmehr im Rahmen einer anderen Fragestellung mit untersucht worden oder dienten als Kontrolle. Diese Ergebnisse sind demnach von den Autoren nicht in Hinblick der Fragestellung dieser Übersichtsarbeit diskutiert worden. Die polysomnografischen Parameter im Nikotinentzug bei Wetter et al. $(1995,1999)$ und einigen anderen Veröffentlichungen sind beispielsweise unter Placebobedingungen bei der Testung von Nikotinpflastern entstanden. Problematisch hierbei könnte sein, dass diese Ergebnisse möglicherweise auch durch die Gabe eines Placebos verfälscht worden sind.

Methodik Des Weiteren unterscheidet sich die Methodik der Studien untereinander z. T. erheblich. Betrachtet man die Forschungsarbeiten über Nichtraucher, denen Nikotin appliziert wurde, variiert zum einen der Zeitpunkt, die Dauer und die Art der Nikotingabe sowie zum anderen die Dosis des Nikotins. Bei Sellini et al. (1989c) wurden beispielsweise Nikotinkaugummis eingesetzt, bei Newhouse et al. (1990) Nikotininfusionen und bei Perkins et al. (2008) Nikotin-Nasenspray. Dasselbe gilt für die tierexperimentellen Arbeiten. Die hierbei resultierenden Dosen, die im Organismus zu Konsequenzen führen könnten, dürfen als völlig unterschiedlich angenommen werden.

Ein weiterer wichtiger Punkt bei Betrachtung der Studien, die die Hormone der HHN-Achse zum Thema haben, sind die unterschiedlichen Zeitpunkte der Messungen. Die Hormonkonzentrationen von Cortisol (bzw. Corticosteron) und/oder ACTH wurden zu unterschiedlichen Zeitpunkten nach Nikotingabe und zu unterschiedlichen Tagezeiten bestimmt. Gerade bei einer zirkadianen Rhythmik von Hormonkonzentrationen mit ihren tageszeitlichen Schwankungen ist der Zeitpunkt der Messung von größter Bedeutung. Hinzu kommt, dass viele Studien über den zeitlichen Aspekt gar keine Auskunft geben.

Auch das Analysenmaterial variiert. Beispielsweise wurde bei Teneggi et al. (2002) das Blut und der Urin untersucht, bei Ceballos und al'Absi (2006) hingegen der Speichel.

Betrachtet man die polysomnografischen Studien, unterscheidet sich auch hier die Technik, insbesondere bei den tierexperimentellen Arbeiten. Hier gibt es große Unterschiede in der technischen 
Art der Ableitung. So variieren Elektrodenart, -anzahl, und/oder - platzierung.

Weitere Aspekte Lässt man die Einschränkungen, die sich aus den Methoden der Studien ergeben (z. B. niedrige Evidenzklasse des Studiendesigns, geringe Probandenzahl, u. a.), außer acht, sind einige wichtige Aspekte zu beachten. Um eine hohe Aussagekraft über ein Thema zu erhalten, müssen möglichst viele Studien gefunden werden, die eine hohe Vergleichbarkeit aufweisen. Mit dieser Voraussetzung wäre die Erstellung einer Metaanalyse möglich, die einen Überblick über die Forschungsergebnisse des untersuchten Themas bietet, z. B. über den Verlauf der Cortisolkonzentration im Nikotinentzug. Diese Voraussetzungen sind bei Betrachtung der Ergebnisse dieser Übersichtsarbeit zu einem großen Teil nicht erfüllt. Dies liegt zum einen an der oft geringen Studienzahl und zum anderen daran, dass die Studien selten über eine homogene Methodik verfügen. Als Beispiel sind die Funde im Abschnitt über die Konsequenzen eines Nikotinentzugs auf dopaminerge Parameter zu nennen. Hier untersucht fast jede der fünf Studien unterschiedliche Teilaspekte dieses Themas: Dagher et al. (2001) konzentrierten sich auf das Bindungspotential an D1-Rezeptoren in der PET, Fehr et al. (2008) hierbei auf die D2- und D3-Rezeptoren. Ward et al. (1991) und Smolka et al. (2004) untersuchten den 24-Stunden-Sammelurin bzw. führten einen ACT durch. Ein gemeinsamer Effekt ist hier somit durch eine Metaanalyse nicht darstellbar. Da diese Übersichtsarbeit einen besonderen Fokus auf den langfristigen Nikotinentzug hat, bieten viele der gefundenen Studien keinen Erkenntnisgewinn, weil oft nur wenige Stunden des Entzugs untersucht wurden. Als Beispiel sei an dieser Stelle ebenfalls auf die Ergebnisse der Literaturrecherche verwiesen, die sich mit Studien über dopaminerge Parameter beschäftigte. In drei von fünf Veröffentlichungen wurde ein Zeitraum von 12 bzw. 24 Stunden analysiert. Ähnlich verhält es sich in den anderen Themenbereichen. Die Konsequenzen eines Nikotinentzugs auf polysomnografische Parameter oder auf den Cortisolspiegel wurden in vielen Studien nur über 48 oder 72 Stunden verfolgt.

\subsection{Anmerkungen zur Literaturrecherche}

In der vorliegenden Arbeit wurde mithilfe einer systematischen Literaturrecherche versucht, die Auswirkungen einer Nikotingabe an Tiere und Nichtraucher sowie eines Nikotinentzugs bei 
Gelegenheitsrauchern und Rauchern auf neuroendokrine und polysomnografische Parameter möglichst umfassend darzustellen. Bei diesem Vorgang sind jedoch einige Einschränkungen zu beachten.

Suchmaschine Zum einen wurde nur in der Datenbank PubMed nach Studien gesucht. Da PubMed die größte und wichtigste Datenbank bezüglich Medizin, Biologie, Psychologie und Biochemie und die primäre Plattform bei der Veröffentlichung von Studienergebnissen darstellt, ist von einem umfassenden Angebot relevanter Studien auszugehen. Doch nicht alle Zeitschriften sind in PubMed gelistet und somit können Veröffentlichungen fehlen.

Stichworte Eine weitere Einschränkung der vorliegenden Arbeit stellt die Auswahl der Stichworte bei der Suche nach Studien dar. Es wurde darauf geachtet, essentielle Suchbegriffe zu finden, die den Themenbereich suffizient abdecken. Dennoch könnten einige Veröffentlichungen andere Begrifflichkeiten verwendet haben und somit konnten diese Veröffentlichungen nicht gefunden werden.

Suchvorgang Bezüglich der Auswahl der Studien aus PubMed ist anzumerken, dass nach den Titeln der Veröffentlichungen beurteilt wurde, ob die Studien relevant für die Übersichtsarbeit sind. Möglicherweise wurden durch dieses Vorgehen die Studien nicht einbezogen, bei denen die Relevanz erst in der Zusammenfassung deutlich wurde. Diesbezüglich ist davon auszugehen, dass Studien, die die Themen der vorliegenden Arbeit nur marginal untersuchten, nicht unbedingt in den Ergebnisteil einbezogen sind.

Sprache Ein letzter Punkt ist, dass einige ausländische Forschungsgruppen ihre Veröffentlichungen nicht in Englisch publizierten. Diese Studien sind in der vorliegenden Arbeit nicht berücksichtigt. 


\subsection{Ergebnisse der Literaturrecherche}

\subsubsection{Objektive Schlafparameter}

\section{Zusammenfassung}

Tierexperimentelle Studien konnten zeigen, dass Nikotin eine dosisabhängige Wirkung auf den Schlaf besaß. Niedrige Dosen erhöhten den REM-Schlaf, höhere Dosen verringerten den REM- und NREM-Schlaf. Bei Nichtrauchern wurde nach Nikotinapplikation eine Abnahme des REM-Schlafs beobachtet. Studien mit Gelegenheitsrauchern wurden nicht gefunden, jedoch Studien, die einen Entzug von Rauchern begleiteten. Hier wurde im akuten Entzug eine stärkere Fragmentierung des Schlafs festgestellt. Eine Nikotinsubstitution besserte den Schlaf insbesondere bei Männern. Die einzige Langzeitstudie zeigte eine Verschlechterung der Schlafqualität und eine Zunahme von depressiven Symptomen im Entzug.

\section{Tierexperimentelle Untersuchungen}

Es wurde eine dosisabhängige Veränderung der polysomnografischen Parameter nach Nikotingabe gefunden. Niedrige Dosierungen verursachten einen Anstieg des REM-Schlafs bei unverändertem (akute Gabe) oder verringertem (chronische Gabe) NREM-Schlaf. Eine höhere Nikotindosis steigerte die Wachheit der Tiere, verbunden mit einem Abfall des REM- und NREM-Schlafs. Die Mechanismen, über die Nikotin die Schlafarchitektur verändert, sind Gegenstand aktueller Forschung und sind zum Teil noch nicht verstanden. Es existiert aber eine Vielzahl von Hypothesen, von denen einige im Folgenden aufgeführt werden.

Nikotin und die Schlaf-Wach-Regulation Viele Autoren schreiben Nikotin über seine aktivierende Wirkung eine generelle Förderung der Wachheit zu. So auch Jewett und Norton (1966), die ebenso wie Domino und Yamamoto (1965) und Vázquez-Palacios et al. (2010) eine Steigerung der Wachheit polysomnografisch erfassten. Die Förderung der Wachheit sei dem Umstand geschuldet, dass Nikotin die Ausschüttung von diversen Transmittern auslöst, die maßgeblich an der Ausbildung des Wachzustandes beteiligt sind. Salín-Pascual et al. (1999) halten die durch Nikotin ausgelöste Freisetzung von Dopamin, Adrenalin, Noradrenalin, Acetylcholin und Serotonin für 
die Ursache der erhöhten Wachzeit nach subkutaner Applikation von Nikotin. Die Freisetzung sei hierbei über die nACh-Rezeptoren erfolgt, da Mecamylamin, ein Antagonist dieser Rezeptoren, die Nikotinwirkung verhinderte. Léna et al. (2004) sprechen sich in diesem Zusammenhang für eine Beteiligung der $\beta 2$-Untereinheit des nACh-Rezeptors aus, da bei Mäusen ohne die genannte Untereinheit die Zunahme der Wachzeit nicht beobachtet wurde.

Der Wachzustand ist u. a. geprägt durch das Überwiegen aminerger und cholinerger Transmitter, die, ausgeschüttet aus Kernen des Hirnstamms, gemeinsam mit dem thalamokortikalen Netzwerk für die charakteristische Wach-EEG verantwortlich sind. Hierbei sind noradrenerge Neurone aus dem Locus coeruleus, cholinerge Neurone aus den Nuclei pedunculopontinus und dorsolateralis tegmentalis und serotonerge Neurone aus den dorsalen Raphekernen mit dem Thalamus und dem Kortex (als Teil des ARAS) verbunden und hemmen den schlaffördernden VLPO des Hypothalamus (Alóe et al., 2005). Dass Nikotin, welches nachgewiesenermaßen in der Lage ist, viele verschiedene Transmitter freizusetzen, in dieses System eingreift und dadurch die Wachheit fördert, ist denkbar. Auch Saint-Mleux et al. (2004) fanden heraus, dass Nikotin die Wachheit fördert, indem es über nACh-Rezeptoren die Freisetzung von Noradrenalin bewirkt und auf diese Weise den VLPO hemmt. Da eine Zunahme der Wachzeit insbesondere nach höheren Dosierungen festgestellt worden ist, scheint ein dosisabhängiger Mechanismus vorzuliegen, bei dem verschiedene Nikotindosen unterschiedliche Auswirkungen auf die Schlaf-Wach-Regulation haben. Dieser Umstand scheint auch für die interne Schlafregulation vorzuliegen.

Nikotin und die interne Schlafregulation Für geringe akute und chronische Dosierungen des Nikotins wurde in vielen Studien eine Erhöhung des REM-Schlafs beobachtet. Die Autoren gehen mehrheitlich davon aus, dass diese Steigerung des REM-Schlafanteils durch die verstärkte Aktivierung des cholinergen Systems hervorgerufen wird, da dieses System den dominierenden Anteil während dieser Schlafphase besitzt. Nikotin fördere direkt über nACh-Rezeptoren oder indirekt über die Freisetzung von ACh (und somit auch über mACh-Rezeptoren) im Hirnstamm die Generierung von REM-Schlaf. Das Gleichgewicht zwischen REM- und NREM-Schlaf wird somit durch Nikotin gestört. Bezieht man das reziproke Interaktionsmodell von McCarley und Hobson (1975) als Veranschaulichung hinzu, wäre dieses Gleichgewicht zugunsten der REM-on- 
Neurone verschoben. Der NREM-Schlaf wird anscheinend bei geringen akuten Nikotindosen nicht beeinflusst.

Höhere akute und chronische Nikotindosen scheinen nicht mehr selektiv das cholinerge System zu beeinflussen, sondern zunehmend auch anderer Transmittersysteme, die in ihrer Gesamtheit die Wachheit fördern und somit den REM- und NREM-Schlaf vermindern.

Salín-Pascual et al. (1999) untersuchten auch den Absetztag nach einer chronischen Nikotingabe. Der unter Nikotin verminderte REM-Schlafanteil erhöhte sich im Nikotinentzug. Die Autoren sprechen in diesem Zusammenhang von einer Erholung (Rebound) des REM-Schlafs. Die Beobachtung einer Erholung machten auch Léna et al. (2004) nach einer akuten Nikotingabe. War der NREM-Schlaf in der ersten Stunde nach Nikotin vermindert und die Wachzeit erhöht, kehrte sich dies in der zweiten Stunde um: Der NREM-Schlaf erhöhte sich, begleitet von einer Abnahme der Wachzeit.

Nikotin und ponto-geniculo-okzipitale Wellen Ein weiterer Aspekt bei der Beeinflussung des Schlafs durch Nikotin sind dessen Wirkungen auf die sogenannten ponto-geniculo-okzipitale (PGO-) Wellen. Diese treten als phasische Erscheinung (d.h. sporadisch und nicht tonisch) kurz vor und während des REM-Schlafs auf und werden interpretiert als die Reaktion auf körperinterne visuelle Orientierungsreize (Schläfke und Schäfer, 2009). Die PGO-Wellen entstehen demnach nicht durch Reize aus der Außenwelt, sondern werden vom ZNS selbst generiert und stimulieren den Kortex, was Erinnerungen und Wahrnehmungen hervorruft - das Träumen (Becker-Carus, 2011).

Man nimmt an, dass die PGO-Wellen in den Nuclei pedunculopontinus und dorsolateralis tegmentalis generiert werden und somit aus cholinergen Neuronen entstehen, die auch für die REM-Schlafgenerierung zuständig sind. Gehemmt werden diese Wellen aminerg durch die dorsalen Raphekerne und den Locus coeruleus (für detailliertere Ausführungen ist auf Shiromani und Gillin (1987) mit den enthaltenen Literaturangaben verwiesen).

In mehreren Veröffentlichungen (Mihailescu et al., 2001; Guzmán-Marín et al., 2001; Vazquez et al., 1996) konnte gezeigt werden, dass Nikotin die PGO-Wellen unterdrückt. Die Autoren dieser 
Studien erklären dies über eine Stimulation der dorsalen Raphekerne durch Nikotin. Die hieraus resultierende Ausschüttung von Serotonin sei für die Inhibition der PGO-Wellen verantwortlich.

Vazquez et al. (1996) sprechen auch die Widersprüche bei den Beobachtungen bezüglich der PGO-Wellen an. Sie zitieren eine Studie von Hu et al. (1988), in der die PGO-Wellen auch durch Mecamylamin blockiert wurden. Somit inhibierten Agonisten und Antagonisten der nACh-Rezeptoren die Generierung von PGO-Wellen. Als mögliche Erklärung hierfür nennen Vazquez et al. (1996) unterschiedliche Angriffspunkte der Substanzen. Ihrer Meinung nach wirke Nikotin über die dorsalen Raphekerne hemmend auf die PGO-Wellen, Mecamylamin hingegen über die direkte Blockierung der PGO-Wellen-generierenden Kerne.

Auch Guzmán-Marín et al. (2001) fallen Widersprüche auf. Zwar halten auch sie die Unterdrückung der PGO-Wellen für das Resultat der Nikotinwirkung an den dorsalen Raphekernen, merken jedoch an, dass diese auch die Kerne hemmen, die den REM-Schlaf generieren. In ihrer Studie wurde allerdings ein Anstieg der REM-Periodendauer beobachtet. Die Autoren erklären dies über eine direkte Aktivierung der REM-on-Neurone durch Nikotin.

\section{Nichtraucher}

Der Einfluss von Nikotin auf den Schlaf und die Stimmung gesunder Nichtraucher Zusammenfassend konnte nach einer Nikotingabe an gesunde Nichtraucher eine Abnahme des REM-Schlafs bei unveränderten NREM-Schlafstadien 3 und 4 und eine unbeeinflusste REM- oder Schlaf-Latenz beobachtet werden. In einer Studie konnte am Absetztag nach der Nikotingabe eine Erholung des REM-Schlafs gemessen werden.

Wie auch in den tierexperimentellen Studien halten die Autoren den Abfall des REM-Schlafanteils für das Resultat der aktivierenden Wirkung des Nikotins, welches somit die Schlafarchitektur und -qualität stört. Davila et al. (1994) und Salín-Pascual et al. (1995) nennen diesbezüglich die durch Nikotin vermehrt freigesetzten Catecholamine, die den Wachzustand fördern. Warum Nikotin in diesem Zusammenhang den REM-Schlaf und nicht die NREM-Schlafstadien 3 und 4 verändert, ist nicht geklärt. Möglicherweise wird selektiv das cholinerge System, welches für die Generierung des REM-Schlafs unerlässlich ist, durch Nikotin inhibiert. Den Widerspruch zu den tierexperimentell 
gewonnenen Ergebnissen, die bei geringen Nikotindosen einen Anstieg des REM-Schlafs zeigten, halten Gillin et al. (1994) beispielsweise für das Ergebnis der unterschiedlichen Methoden. Sie verweisen hierbei auf die verschiedenen Arten der Nikotinverabreichung und den Einsatz von unterschiedlichen Spezien.

Salín-Pascual und Drucker-Colín (1998) schreiben in ihrer Veröffentlichung, dass sie nach Studium der bisherigen Studien zu den Konsequenzen einer Nikotingabe an Nichtraucher überrascht waren, einen Anstieg des REM-Schlafs ab der zweiten Nacht unter Nikotin beobachtet zu haben, welcher sich auch noch nach vier Tagen Nikotin und sogar verstärkt im darauffolgenden Nikotinentzug zeigte. Sie halten es für möglich, dass durch die chronische Nikotingabe eine Sensibilisierung des REM-Schlafauslösenden Systems stattfand, woraufhin sich der REM-Schlaf erhöhte.

Eine Erholung der veränderten Schlafparameter im Nikotinentzug konnte in der Studie von Gillin et al. (1994) beobachtet werden, in der der REM-Schlaf und das NREM-Schlafstadium 2 in der Absetznacht kompensatorisch erhöht bzw. vermindert waren.

Gillin et al. (1994) konnten keine stimmungsverbessernden Wirkungen des Nikotins feststellen, was im Widerspruch zu den Veröffentlichungen von Salín-Pascual et al. (1995) und Salín-Pascual und Drucker-Colín (1998) steht (s. u.).

\section{Der Einfluss von Nikotin auf den Schlaf und die Stimmung depressiver Nichtraucher}

Bei den Studien, die Probanden mit Depressionen untersuchten, fiel auf, dass im Gegensatz zu den gesunden Probanden mehrheitlich ein Anstieg des REM-Schlafs beobachtet wurde. Andere polysomnografische Parameter waren nach einer akuten Nikotingabe nicht wesentlich beeinflusst. Eine chronische Applikation von Nikotinpflastern ließ die REM-Latenz, die Gesamtschlafzeit und den Tiefschlaf ansteigen. In allen Studien besserten sich die Depressionssymptome.

Der Zusammenhang zwischen Nikotinkonsum und Depressionen sowie zwischen Depressionen und Schlaf ist in vielen Veröffentlichungen belegt worden. So stellten Glassman et al. (1990) eine erhöhte Raucherquote bei Menschen mit Depressionen fest und dass diese bei einem Abstinenzversuch weniger erfolgreich waren. Auch gelten Schlafstörungen als ein wichtiges Symptom bei 
Depressionen. Es wird von frühmorgendlichem Erwachen und einem verminderten Tiefschlafanteil gesprochen (Kotterba und Schäfer, 1999). Besonders charakteristisch für eine Depression scheinen jedoch Veränderungen der REM-Schlafparameter zu sein. Diese zeichnen sich durch einen verlängerten REM-Schlaf und eine verkürzte REM-Latenz aus (Hajak und Rüther, 1995), die möglicherweise aus einer erhöhten cholinergen Aktivität im ZNS von Depressiven hervorgeht (die REM-Latenz wird als Maßstab für die Aktivität des cholinergen Systems angesehen) (Salín-Pascual, 2002).

Doch nicht nur das cholinerge System scheint bei Menschen mit Depressionen verändert zu sein, auch das aminerge System ist beeinflusst. Möglicherweise ist der bei Depressionen vorliegende Mangel an Serotonin ursächlich für die verminderte Inhibition des REM-Schlafs durch die dorsalen Raphekerne (Jähne et al., 2010). Aus diesen veränderten Aktivitäten der Transmittersysteme resultiert demnach eine Störung der Balance zwischen den aminergen und cholinergen Transmittern (Janowsky et al., 1972), was zu den oben genannten Veränderungen der polysomnografischen Parameter führt.

Zusammenfassend kann festgehalten werden, dass Nikotin bei depressiven Probanden nach akuter Applikation den REM-Schlaf erhöhte und während einer chronischen Gabe die REM-Latenz verlängerte. Diesem scheinbaren Widerspruch - Förderung und Inhibition des cholinergen Systems durch Nikotin - begegnen Jähne et al. (2010) mit der Hypothese, dass eine akute Nikotingabe primär aktivierend auf das cholinerge System wirkt und somit den REM-Schlaf erhöht, eine chronische Gabe jedoch vermehrt das serotonerge System anspricht, was zu einer Inhibition des REM-Schlafs und einer verlängerten REM-Latenz führt. Auch die Autoren der Studien dieser Übersichtsarbeit nehmen an, dass die durch Nikotin aufgetretene Verlängerung des REM-Schlafs möglicherweise auf die bei Depressiven vorkommende Hypersensitivtät des cholinergen Systems zurückzuführen ist. Dieser Meinung sind auch Salín-Pascual und Galicia-Polo (1999), die in ihrer Studie ebenfalls eine Erhöhung des REM-Schlafs und eine Verbesserung der Depressionen beobachten konnten.

Der Zusammenhang zwischen REM-Schlafparametern und Stimmung ist von Haro und DruckerColín (2004) gezeigt worden. In ihrer Untersuchung konnte die durch Nikotin normalisierte REMLatenz mit einer Verbesserung der Depressionssymptomatik in Verbindung gebracht werden. Auch Salín-Pascual (2002) konnte zeigen, dass insbesondere Probanden mit einer geringen REM-Latenz 
vor der Nikotingabe eine Besserung der Depressionen durch Nikotin erfuhren.

Haro und Drucker-Colín (2004) konnten in ihrer Langzeitstudie neben der Besserung der Depressionen eine deutliche Verbesserung der bei Depressiven gestörten Schlafarchitektur beobachten. Die Gesamtschlafzeit, die Schlaf-Effizienz und der Tiefschlafanteil stiegen an. Die Autoren sehen hierbei einen Zusammenhang mit dem serotonergen System, da Tryptophan, eine Aminosäure, aus der Serotonin synthetisiert wird, den Tiefschlaf fördert, die Gesamtschlafzeit verlängert und in zu geringer Konzentration Depressionen auslösen kann. Die bei depressiven Patienten verringerten Tryptophanspiegel (Song et al., 1998) werden somit durch Nikotin erhöht, was dazu führt, dass sich die Depressionen und die Schlafarchitektur verbessern. Die Autoren resümieren, dass Nikotin für die Behandlung von Depressionen geeignet ist.

\section{Raucher}

Vor der Interpretation der polysomnografischen Veränderungen im Nikotinentzug ist wichtig zu erfahren, inwieweit der Schlaf von Rauchern vor der Abstinenz im Gegensatz zu Nichtrauchern verändert war. Hierzu liegen einige Studien vor, die bei Jähne et al. (2010) in einer Übersicht aufgeführt sind.

Unterschiede zwischen Rauchern und Nichtrauchern bezüglich des Schlafs Viele Veröffentlichungen haben gezeigt, dass Raucher häufiger unter Schlafstörungen leiden als Nichtraucher. Wetter und Young (1994) konnten beispielsweise anhand von Fragebögen die subjektiven Beschwerden von Rauchern und Nichtrauchern ermitteln und stellten fest, dass Raucher stärkere Probleme mit dem Einschlafen und Aufwachen hatten als Nichtraucher. Die Raucherinnen klagten zudem vermehrt über Tagesmüdigkeit, die männlichen Raucher hingegen über Albträume. Auch Cohrs et al. (2012) stellten im Rahmen der German Multicenter Study on Nicotine Dependence eine geringere subjektive Schlafqualität, eine erhöhte Schlaf-Latenz und eine geringere Schlafdauer bei Rauchern im Gegensatz zu Nichtrauchern fest. Insbesondere starke Raucher klagten vermehrt über Schlafstörungen.

Objektive Unterschiede in der Schlafqualität zwischen Rauchern und Nichtrauchern konnten von Soldatos et al. (1980) und Zhang et al. (2006, 2008) beobachtet werden. Soldatos et al. (1980) 
konnten zwar keine Unterschiede in den Parametern der Schlafstadien zwischen Rauchern und Nichtrauchern ausmachen, jedoch eine erhöhte Schlaf-Latenz und Wachzeit bei Rauchern messen. Die letzten beiden Punkte wurden auch von Zhang et al. (2006) bestätigt. In ihrer Studie wurden zudem noch folgende Unterschiede aufgedeckt: Raucher wiesen einen erhöhten Anteil der NREM-Schlafstadien 1 und 2 und einen verminderten Tiefschlafanteil auf sowie eine geringere Schlaf-Effizienz als Nichtraucher. Die bei Rauchern aufgetretene Verschiebung zugunsten des leichteren Schlafs wurde von derselben Forschungsgruppe zwei Jahre später (Zhang et al., 2008) mittels Spektralanalyse des Schlafs bestätigt. Insbesondere in den ersten Schlafphasen waren die Unterschiede zwischen Rauchern und Nichtrauchern zu beobachten. Den Autoren zufolge spreche dies für den Einfluss des Nikotins auf den Schlaf, da die Nikotin-Blutkonzentration in dieser Phase am höchsten ist und im Laufe des Schlafens sinkt.

Die Gründe für die subjektiv empfundene und objektiv gemessene schlechtere Schlafqualität der Raucher scheinen vielfältig zu sein und sind Gegenstand aktueller Forschung. Zhang et al. (2006, 2008) und Soldatos et al. (1980) nehmen u. a. an, dass die durch Nikotin ausgelöste Ausschüttung diverser Neurotransmitter für die Beeinflussung des Schlafprofils ursächlich ist. Wie bereits bei den Studien mit Tieren und Nichtrauchern erwähnt wurde, scheint Nikotin auch bei Rauchern die Wachheit zu fördern. Interessanterweise war diesbezüglich bei Rauchern der Tiefschlaf vermindert, bei Nichtrauchern nach Nikotinapplikation hingegen der REM-Schlaf. Die Studien erklären ihre Beobachtungen mit ähnlichen Argumenten: Nikotin fördere die Wachheit aufgrund der Freisetzung der unterschiedlichsten Botenstoffe zum einen auf Kosten des Tiefschlafs, zum anderen auf Kosten des REM-Schlafs. Beachtet werden muss hierbei, dass den Nichtrauchern Nikotin nur über wenige Tage verabreicht wurde und dass ein genereller Vergleich zwischen Nichtrauchern nach Nikotingabe und chronischen Rauchern sehr schwierig ist.

Ein weiterer Grund für den gestörten Schlaf von Rauchern ist möglicherweise die Beeinträchtigung des Schlafs durch die während der Schlafphase auftretenden Entzugserscheinungen (Zhang et al., 2006). Die steigende Anzahl unbesetzter Nikotinrezeptoren führt zu einem Ungleichgewicht im cholinergen System, was z. B. körperlichen Stress und Unruhe auslöst und möglicherweise den Schlaf negativ beeinträchtigt.

Zhang et al. (2006) halten die vielfältigen gesundheitsschädlichen Folgen des Rauchen ebenfalls 
für eine Ursache für den gestörten Schlaf von Rauchern. Beispielhaft sei an dieser Stelle auf die zahlreichen Lungenerkrankungen verwiesen, die durch den Tabakkonsum ausgelöst werden, die wiederum den Schlaf stören können.

Die beobachteten Unterschiede in der Schlafqualität zwischen Rauchern und Nichtrauchern scheinen reversibel zu sein. In der Veröffentlichung von Zhang et al. (2006) konnten keine Unterschiede in der Schlafarchitektur zwischen Nichtrauchern und ehemaligen Rauchern entdeckt werden.

Der Einfluss eines mehrtägigen Nikotinentzugs auf den Schlaf Die Studien über einen mehrtägigen Entzug erbrachten keine eindeutigen Ergebnisse. Zwei unabhängige Forschungsgruppen (die von G. Prosise und D. Wetter) haben jedoch eine erhöhte Fragmentierung des Schlafs während der ersten drei Entzugsnächte festgestellt. Wetter et al. (2000) halten die Fragmentierung des Schlafs, welche gekennzeichnet ist durch häufigere Stadienwechsel und Arousals, für ein wichtiges Kriterium bei der Beurteilung der Schlafqualität und für ein wichtiges, valides Entzugssymptom. Durch welche Mechanismen die Fragmentierung entsteht ist nicht bekannt.

Schlaf und Nikotinersatztherapien Da der im Entzug gestörte Schlaf z. B. durch eine verstärkte, belastende Tagesmüdigkeit das Rückfallrisiko erhöhen kann, ist der Nutzen einer Nikotinersatztherapie auch bezüglich polysomnografischer Parameter untersucht worden.

Wetter et al. $(1995,1999)$ haben Nikotinpflaster eingesetzt und kamen zu dem Ergebnis, dass der Schlaf im Entzug durch die Nikotinsubstitution verbessert wurde (insbesondere bei Männern). Die Fragmentierung nahm unter Nikotin ab und der Tiefschlafanteil stieg an. In Wetter et al. (1999) analysierte die Forschungsgruppe die Ergebnisse von Männern und Frauen getrennt voneinander. Sie stellte fest, dass die Männer eher von einer Nikotinersatztherapie profitierten als die Frauen. Waren zum Zeitpunkt der Baseline-Messungen die Parameter der Schlaffragmentierung zwischen beiden Geschlechtern gleich, zeigte sich im Entzug eine geringere Schlaf-Effizienz und eine höhere Wachzeit bei Frauen unter Nikotin. Die Männer hingegen erwachten unter der Verwendung von Nikotinpflastern seltener. Die Gründe für die beobachteten Unterschiede sind nicht bekannt - auch nach der Kontrolle von Einflussfaktoren wie Alter, Nikotinabhängigkeit oder Body-Mass-Index (BMI) waren die Unterschiede signifikant. 
Die bei Wetter et al. $(1995,1999)$ verwendeten Nikotinpflaster hatten die Dosierung von $22 \mathrm{mg}$ Nikotin und wurden über 24 Stunden getragen. Staner et al. (2006) und Aubin et al. (2006) verglichen den Nutzen von Nikotinpflastern unterschiedlicher Dosierung und Tragezeit auf den Schlaf im Nikotinentzug miteinander. Sie kamen zu dem Ergebnis, dass Nikotinpflaster, die über 24 Stunden getragen wurden, den Schlaf noch mehr verbesserten als eine Tragezeit von 16 Stunden. Dies zeigte u. a. das Auftreten eines erhöhten Tiefschlafanteils und die geringere Schlaf-Fragmentierung unter Verwendung der 24-Stunden-Nikotinpflaster. Zudem war unter dieser Dosierung die Dichte des REM-Schlafs erhöht und dies könnte für eine stärkere Traumaktivität sprechen. Auch Page et al. (2006) fanden heraus, dass die Intensität der Träume im REM-Schlaf unter Nikotinpflastern erhöht war.

Doch auch die Nikotinersatztherapie scheint ebenso wie der Nikotinentzug Schlafstörungen auszulösen. So berichteten die Probanden bei Fredrickson et al. (1995) und der Imperial Cancer Research Fund General Practice Research Group (1993) von Schlafproblemen, wie z. B. Ein- und Durchschlafschwierigkeiten. Auch objektiv konnte beispielsweise bei Page et al. (2006), Staner et al. (2006) und Aubin et al. (2006) ein gestörter Schlaf nach Applikation von Nikotinpflastern beobachtet werden. Eine verlängerte Wachzeit, mehr Arousals, eine geringere Schlaf-Effizienz und eine erhöhte Schlaf-Latenz waren das Resultat der Verwendung von Nikotinpflastern.

Der Einfluss eines Langzeit-Nikotinentzugs auf den Schlaf Eine einzige Studie untersuchte den Langzeitverlauf eines Nikotinentzugs polysomnografisch. Moreno-Coutiño et al. (2007) stellten neben einer Abnahme des Tiefschlafs und der Schlaf-Effizienz eine Zunahme des REM-Schlafs und eine Abnahme der REM-Latenz während einer zwölfmonatigen Entzugszeit fest. Zudem erhöhte sich der Punktwert des HAM-Ds. Die Autoren postulieren, dass Raucher durch den Nikotinkonsum im Sinne einer Selbstmedikation depressive Symptome kontrollieren. Im Entzug zeigte sich eine Zunahme der Depressionen und ein Überwiegen der cholinergen Aktivität, was sich durch den erhöhten REM-Schlafanteil darstellte, der für depressive Menschen charakteristisch ist (s. o.). Zudem schreiben sie Serotonin eine entscheidende Rolle bei den beobachteten Veränderungen im Entzug zu. Durch den Wegfall der durch Nikotin ausgelösten Serotoninausschüttung im Entzug sei die vermehrte Ausbildung depressiver Symptome zustande gekommen. 
Depressionen und Nikotinentzug Eine weitere Studie untersuchte neben gesunden auch depressive Probandinnen während eines zehntägigen Entzugs (Wetter et al., 2000). Der Vergleich beider Gruppen zeigte, dass sich die Ergebnisse der Baseline-Messungen beider Gruppen ähnelten, sich im Entzug jedoch in den REM-Schlafvariablen unterschieden. Blieb der REM-Schlafanteil bei Gesunden im Entzug gleich, stieg er in der depressiven Gruppe nicht signifikant an. Die REM-Latenz änderte sich im Gegensatz zu den gesunden Probandinnen nicht, welche in den ersten Tagen eine verlängerte REM-Latenz aufwiesen. Die Studienresultate passen nach Meinung der Autoren zu bisherigen Erkenntnissen, dass Stress bei gesunden Menschen zu einer erhöhten REMLatenz führen kann, depressive Menschen hingegen wenig empfänglich sind für stressinduzierte Veränderungen der REM-Latenz.

Die von den Probandinnen subjektiv eingeschätzte Schlafqualität unterschied sich zwischen den gesunden und depressiven Studienteilnehmerinnen kaum. Die Autoren resümieren, dass die objektiv bestimmten REM-Schlafvariablen sehr sensitive Parameter darstellen, wenn es darum geht, Unterschiede im Verlauf des Nikotinentzugs zwischen Menschen mit und ohne affektive Störung aufzudecken, die durch eine subjektive Beurteilung des Schlafs nicht in Erscheinung treten.

Objektive Messergebnisse und subjektive Bewertungen des Schlafs Interessanterweise stimmten in den Studien die objektiven Messergebnisse oft nicht mit den von den Probanden geschilderten Symptomen überein. So bewerteten die Studienteilnehmer mit Nikotin- oder Placebopflaster bei Wetter et al. $(1995,1999)$ die Schlafqualität im Entzug annähernd gleich. Der objektiv verbesserte Schlaf unter der Verwendung von Nikotinpflastern spiegelte sich demnach nicht in den subjektiv empfundenen Variablen wider. Dies lässt auf die Vielzahl der Komponenten schließen, die bei der Entstehung der subjektiven Einschätzung des Schlafs beteiligt sind, und lässt die Verwertbarkeit der subjektiven Parameter als Maß für die Schlafqualität anzweifeln - oder andersherum: Die objektiv gemessenen Schlafparameter sind anscheinend nicht in der Lage, das subjektive Empfinden widerzuspiegeln. 


\subsubsection{Dopaminerges System}

\section{Zusammenfassung}

Es konnten keine Studien über die Auswirkungen einer Nikotingabe an Nichtraucher sowie über die Konsequenzen eines Nikotinentzugs bei Gelegenheitsrauchern gefunden werden. Fünf Veröffentlichungen beschäftigten sich mit dem Nikotinentzug von Rauchern. Das bei Rauchern im Gegensatz zu Nichtrauchern verminderte Bindungspotential an striatale Dopaminrezeptoren in der PET sowie eine geringere Sensitivität der D2-Rezeptoren blieb auch in den ersten 12 bzw. 24 Stunden eines Entzugs erhalten. Im längerfristigen Entzug nahm das Bindungspotential an D1-Rezeptoren des Striatums zu und die Dopaminausscheidung über den Urin ab.

\section{Nichtraucher}

Durch die Literaturrecherche sollten Studien gefunden werden, in denen Nichtrauchern Nikotin verabreicht wurde und die Folgen für das dopaminerge System untersucht wurden. Es konnten allerdings mit den verwendeten Stichwörtern keine Studien gefunden werden, die sich mit diesem Thema beschäftigten.

Ergebnisse tierexperimenteller Arbeiten Die Folgen einer Nikotingabe auf das dopaminerge System nikotinnaiver Lebewesen sind tierexperimentell zum Teil gut untersucht. Aus diesen Ergebnissen, die sicher nicht eins zu eins auf den Menschen übertragen werden können, lassen sich Hinweise auf die Wirkung des Nikotins auf das dopaminerge System finden. Einige Ergebnisse dieser Arbeiten sind im Folgenden dargestellt.

Bei Ratten wurde ein dosisabhängiger Anstieg der Dopaminkonzentration im Nucleus accumbens nach subkutaner Nikotinapplikation beobachtet. Eine der Nikotingabe vorausgehende Applikation des Stoffes Mecamylamin (ein zentraler Antagonist der nACh-Rezeptoren) konnte die Dopaminausschüttung blockieren, was darauf schließen lässt, dass die erhöhten Dopaminkonzentrationen aus einer Aktivierung der nACh-Rezeptoren resultierten (Imperato et al., 1986). Eine Toleranz stellte sich auch nach mehrtägiger Nikotinapplikation nicht ein, es konnten dieselben erhöhten Dopaminkonzentrationen wie nach einer akuten Gabe beobachtet werden (Damsma et al., 1989). 
Pontieri et al. (1996) zeigten, dass sich die Dopaminausschüttung nach intravenöser Nikotingabe bei Ratten auf die Schalenregion des Nucleus accumbens konzentrierte, während in der Kernregion keine signifikant erhöhten Dopaminwerte gemessen wurden.

Zhang et al. (2012) haben in ihrer Veröffentlichung u. a. die Dopaminkonzentration in der Schalenregion des Nucleus accumbens bei Mäusen gemessen, die vier bzw. zwölf Wochen Nikotin über das Trinkwasser erhielten. Der darauf folgende Entzug über zehn Tage war Gegenstand der Studie. Am ersten Entzugstag zeigte sich eine gegenüber der Kontrolle (Mäuse, die kein Nikotin erhalten hatten) verminderte Dopaminausschüttung im Nucleus accumbens. Diese geringere Dopaminkonzentration blieb unterschiedlich lang, von der Dauer der Nikotinapplikation abhängig, erhalten: Mäuse, die vier Wochen Nikotin bekamen, wiesen an Tag fünf des Entzugs ähnliche Dopaminwerte auf wie die Kontrolle, wohingegen die Mäuse mit zwölfwöchiger Nikotinapplikation an diesem Tag geringere Werte aufwiesen als am ersten Entzugstag. Erst nach zehn Tagen ohne Nikotin normalisierte sich die Dopaminkonzentration auch in dieser Gruppe. Neben diesen Untersuchungen wurde auch eine Art Rückfall simuliert. Im Entzug wurde den Mäusen Nikotin verabreicht und die resultierenden Dopaminkonzentrationen gemessen. Der zuvor hypodopaminerge Zustand wurde durch eine erneute Nikotingabe aufgehoben und interessanterweise war die Dopaminkonzentration nun höher als bei der Kontrolle.

Diese Ergebnisse zusammengenommen sprechen für eine nikotin-induzierte Aktivierung des Belohnungssystems, welche sich durch eine erhöhte Dopaminausschüttung insbesondere in der Schalenregion des Nucleus accumbens äußert. Diese Wirkung scheint aus einer Besetzung der nACh-Rezeptoren auf den Zellen des Belohnungssytems zu resultieren. Zudem unterliegt die dopaminerge Antwort auf Nikotin nach Damsma et al. (1989) keiner Toleranz: Chronische Nikotingaben riefen dieselben Belohnungseffekte hervor wie eine akute Gabe. Es konnte außerdem gezeigt werden, dass es im Entzug zu einem dosisabhängigen, hypodopaminergen Zustand kam, der durch einen Rückfall aufgehoben wurde. In dieser Situation wurde beobachtet, dass das dopaminerge System empfindlicher auf die erneute Nikotingabe reagierte und somit vermutlich eine ausgeprägtere Verstärkung des Konsums stattfand. 


\section{Raucher}

\section{Unterschiede zwischen Rauchern und Nichtrauchern bezüglich des dopaminergen Sys-}

tems Drei der gefundenen Studien untersuchten mithilfe der PET die Ligandenbindung an zentrale Dopaminrezeptoren (Dagher et al. (2001) und Yasuno et al. (2007) konzentrierten sich auf D1-, Fehr et al. (2008) auf D2- und D3-Rezeptoren). Diesen Veröffentlichungen gemein ist, dass sie Raucher und Nichtraucher miteinander verglichen und somit Unterschiede im dopaminergen System zwischen diesen beiden Probandengruppen aufdeckten.

Es lässt sich feststellen, dass Raucher im Vergleich zu nicht rauchenden Probanden ein geringeres Bindungspotential an die untersuchten Dopaminrezeptoren aufwiesen. Die Unterschiede waren nach Yasuno et al. (2007) und Fehr et al. (2008) auf bestimmte Hirnregionen beschränkt. So beobachteten beide Forschungsgruppen diese Unterschiede nur in Teilen des Striatums. Auch Dagher et al. (2001) konnten das geringere Bindungspotential in dieser Hirnstruktur bestätigen (sie hatten sich mit der PET auf dieses Areal beschränkt).

Die Autoren halten es für möglich, dass die durch Nikotin vermehrte Dopaminausschüttung ursächlich ist für diese Veränderung. Sie gehen von einer kompensatorischen Down-Regulation der Dopaminrezeptoren als Antwort auf die chronisch erhöhten Dopaminkonzentrationen aus. Diese Unterfunktion des postsynaptischen dopaminergen Systems wurde schon für den chronischen Konsum anderer Drogen gezeigt, z. B. Alkohol (Volkow et al., 1996), Kokain (Volkow et al., 1997) und Heroin (Wang et al., 1997). Die Down-Regulation der Dopaminrezeptoren könnte ein Grund für die Aufrechterhaltung des Nikotinkonsums darstellen, da laut Yasuno et al. (2007) und Fehr et al. (2008) das Verlangen der Probanden nach Nikotin mit der Dichte der Dopaminrezeptoren korreliert: Yasuno et al. (2007) beobachteten eine negative Korrelation im Nucleus accumbens (D1Rezeptoren), das heißt, je geringer die Dichte der Rezeptoren dort, desto größer das Verlangen. Fehr et al. (2008) zeigten diese Verknüpfung in anderen Hirnregionen für die D2- und D3- Rezeptoren (allerdings nicht im Nucleus accumbens, hier wurde im Gegensatz zu den Erwartungen der Autoren eine positive Korrelation beobachtet). Diese Wechselbeziehung zwischen Verlangen und dem Bindungspotential an Dopaminrezeptoren in der PET ist auch in der Forschung über Alkoholabhängigkeit bekannt. Heinz et al. (2004) z. B. beobachteten, dass das Verlangen der 
Probanden nach Alkohol umso höher ausfiel, je geringer das Bindungspotential an D2-Rezeptoren im ventralen Striatum war.

Das geringere Bindungspotential an Dopaminrezeptoren in der PET bei Rauchern deuten die Autoren meist als eine Down-Regulation der Anzahl oder Dichte der Rezeptoren. Smolka et al. (2004) haben hingegen mithilfe des ACTs die Funktion der Dopaminrezeptoren untersucht und die hormonelle Antwort auf eine Bindung an diese gemessen. Diese war bei den Rauchern im Verhältnis zu den nichtrauchenden Probanden erniedrigt, wobei hierfür keine eindeutige Aussage über die Ursache gemacht werden kann, da entweder auch die Anzahl erniedrigt oder die Funktion beeinträchtigt war. Auch die Autoren dieser Studie halten diese Unterschiede für eine Folge des Nikotinkonsums, da ihre Ergebnisse mit der Nikotinabhängigkeit korrelieren: Je höher die Stärke der Abhängigkeit, desto geringer die hormonelle Antwort (Sensitivität).

Nikotinabhängigkeit, dopaminerges System und Genetik Eine andere Hypothese der Autoren hält die beobachteten Unterschiede nicht für die Folgen, sondern für die Ursache des Rauchens. Eine möglicherweise genetisch bedingte Unteraktivität des dopaminergen Systems wäre dann ein prädestinierender Faktor für die Entwicklung einer Nikotinabhängigkeit. Eine genetische Komponente der Abhängigkeitsentwicklung ist in der Suchtforschung ein vieldiskutiertes Thema, so gehen einige Forschungsarbeiten über den Einfluss von genetischen Faktoren in der Alkoholabhängigkeit davon aus, dass über $60 \%$ der Suchtentstehungsfaktoren genetischen Ursprungs sind (Heath et al., 1997). Ähnliche Prozentzahlen können in Studien über die Nikotinabhängigkeit gefunden werden. McGue et al. (2000) geben an, Tabakkonsum und Nikotinabhängigkeit seien zu $40-60 \%$ hereditär.

Eine Übersicht über mögliche Kandidatengene, die bei der Entwicklung einer Nikotinabhängigkeit eine Rolle spielen könnten, bietet z. B. die Veröffentlichung von Yoshimasu und Kiyohara (2003). Hierin werden auch die Polymorphismen der Dopaminrezeptoren diskutiert und im Falle des D2-Rezeptors eine interessante Entdeckung beschrieben: Für das Gen, das für die Kodierung der D2-Rezeptoren zuständig ist, liegen zwei unterschiedliche Allele vor (A1 und A2). Menschen mit Suchterkrankungen und Übergewicht tragen häufiger das Allel A1. Dieses ist mit einer geringeren Anzahl an D2-Rezeptoren assoziiert, was für den Träger eine angeborene Unterfunktion 
des Dopaminsystems bedeuten könnte. Menschen mit dem Allel A1 sind also möglicherweise prädestiniert für die Entwicklung einer Sucht, da sie durch den Konsum von z. B. Nikotin eine stärkere Belohnung verspüren als Menschen ohne diese Unterfunktion (Noble et al., 1994).

Der Einfluss eines Nikotinentzugs auf das dopaminerge System Wie auch immer sich die bestehenden Unterschiede zwischen Rauchern und Nichtrauchern erklären lassen, sie geben in jedem Fall einen Hinweis auf mögliche Faktoren, die einen erfolgreichen Entzug erschweren. Interessanterweise scheinen die bei Rauchern vorliegenden Unterschiede einer gewissen Stabilität zu unterliegen, da laut der gefundenen Veröffentlichungen in den ersten 12 bis 24 Stunden keine Veränderungen der gemessenen Parameter auftraten. Eine einzige Studie untersuchte mithilfe der PET auch den längerfristigen Abstinenzverlauf und konnte eine Zunahme des Bindungspotentials an D1-Rezeptoren beobachten (Yasuno et al., 2007). Dieser Umstand könnte als eine Art Erholung oder Restitution gedeutet werden, wenn man davon ausgeht, dass Nikotin für die Down-Regulation der Rezeptoren verantwortlich war. Gleichzeitig verminderte sich im Laufe der Abstinenz das Verlangen nach Nikotin, was die bereits erwähnte Aussage über die Entdeckung einer Korrelation zwischen diesem Verlangen und dem Bindungspotential an striatale D1-Rezeptoren unterstützt.

Ward et al. (1991), deren Studie sich erheblich von den bisher erwähnten Veröffentlichungen unterscheidet, beschäftigten sich mit der peripheren Dopaminkonzentration im längerfristigen Nikotinentzug. Sie untersuchten die Dopaminkonzentration im Urin von abstinenten Rauchern und fanden - unabhängig von der vorherigen Stärke des Tabakkonsums - einen deutlichen Konzentrationsabfall über einen Entzugszeitraum von 30 bzw. 90 Tagen. Dieses Ergebnis interpretieren die Autoren als Resultat des Wegfalls der chronischen Aktivierung des sympathischen Nervensystems. Ein von anderen physiologischen Parametern bekanntes Phänomen, der Rebound, wurde im Versuchszeitraum nicht beobachtet. Gemeint ist ein vorübergehender, der Nikotinwirkung entgegengesetzter Effekt, hier ein kurzes Absinken der Dopaminkonzentration, auf welches ein Ansteigen auf Werte wie vor dem Entzug folgt.

Lediglich eine Studie gab Auskunft über periodische Beinbewegungen während des Entzugs. Prosise et al. (1994) beobachteten eine nicht signifikante Erhöhung des PLM-Indexes. Einen Zusammenhang von PLM mit einem erhöhten Rückfallrisiko, wie es für die Alkoholabhängigkeit 
gezeigt wurde (Gann et al., 2002), konnte für die Nikotinabhängigkeit nicht festgestellt werden.

\subsubsection{Hypothalamus-Hypophysen-Nebennieren-Achse}

\section{Zusammenfassung}

Die tierexperimentellen Arbeiten ergaben, dass die Hormone der HHN-Achse nach akuter Nikotingabe anstiegen. Wurde Nikotin chronisch verabreicht, wurde zumindest ein temporärer Anstieg beobachtet. Ein Entzug bei den Versuchstieren erbrachte keine eindeutigen Ergebnisse. Nach Nikotingabe an Nichtraucher konnte ebenfalls eine Erhöhung der Cortisol- und ACTH-Konzentration gemessen werden. Studien, die einen Entzug bei Gelegenheitsrauchern untersuchen, konnten nicht gefunden werden, jedoch einige, die einen Entzug von Rauchern begleitet haben. Diese konnten zeigen, dass sich die Konzentrationen der Hormone der HHN-Achse während der ersten Entzugstage nicht wesentlich änderten, in der längerfristigen Abstinenz jedoch sanken.

\section{Tierexperimentelle Untersuchungen}

Alle bis auf eine (Altland et al., 1978) der 24 Studien, in denen Tiere einmalig Nikotin appliziert bekamen, stellten einen Anstieg der gemessenen Hormone der HHN-Achse fest. Mit zwei Ausnahmen (Seifert et al., 1984; Morse, 1989) konnte in allen Studien, in denen Tieren Nikotin über mehrere Tage bzw. Wochen gegeben wurde, zumindest ein temporärer Anstieg der Hormone der HHN-Achse beobachtet werden.

Lokalisation der Nikotinwirkung Wodurch diese erhöhten Hormonwerte nach Nikotingabe zustande kamen, war z. T. Gegenstand der Untersuchungen. Matta et al. (1987) gingen z. B. der Frage nach, ob die nikotin-induzierte Erhöhung der HHN-Hormone durch einen peripheren oder zentralen Mechanismus zustande kommt. Hierfür setzten sie Cytisin ein, einen Agonisten der nACh-Rezeptoren, der die Bluthirnschranke im Gegensatz zu Nikotin nur in geringem Maße überwinden kann. Es stellte sich heraus, dass nur Nikotin, aber nicht Cytisin einen Anstieg des ACTH-Wertes im Blut bewirkte. Ebenso konnte ein peripherer Antagonist der nACh-Rezeptoren (Hexamethonium) den nikotin-induzierten Anstieg des ACTHs im Blut nicht verhindern, jedoch 
ein zentraler Antagonist der nACh-Rezeptoren (Mecamylamin). Diese Ergebnisse sprechen für einen zentralen Wirkungsmechanismus, schließen jedoch periphere Wirkungen nicht aus. Turner (1975) hingegen postuliert, dass die zentrale Wirkung des Nikotins nicht signifikant sei und die nikotin-induzierte Corticosteron-Sekretion über eine Ausschüttung von Catecholaminen des Nebennierenmarks (NNM) zustande komme. Dies schloss er aus seiner Untersuchung, in der der Corticosteron-Anstieg im Blut der Ratten nach Nikotin nicht stattfand, wenn er das NNM der Tiere entfernte, jedoch gestresste Tiere ohne NNM weiterhin erhöhte Corticosteron-Werte aufwiesen. Caggiula et al. (1991) nehmen an, dass zentrale und periphere Mechanismen an der Nikotinwirkung auf die HHN-Achse beteiligt sind, die zentralen aber überwiegen. Hierfür spricht auch die breite Verteilung der nACh-Rezeptoren in peripheren und zentralen Strukturen und möglicherweise wirken beide Mechanismen synergistisch.

Suzuki et al. (1973) und Cam et al. (1979) entnahmen in ihren Studien den Versuchstieren die Hypophysen und verabreichten den Tieren Nikotin. Der zuvor an den nicht operierten Tieren festgestellte Hormonanstieg des Corticosterons blieb aus, was die Schlussfolgerung zulässt, dass der Corticosteron-Anstieg durch eine Nikotinwirkung an der oder über die Hypophyse zustande kommt. Eine direkte Nikotinwirkung an der Hypophyse ist jedoch umstritten. Matta et al. (1987) konnten zeigen, dass Nikotin in vitro keinen Einfluss auf die Sekretion von $\beta$-Endorphin aus der Hypophyse hat, einem Peptid, das zusammen mit ACTH aus dem gemeinsamen Vorläuferprotein Proopiomelanocortin entsteht. Moidel et al. (2006) hingegen beobachteten einen Anstieg der ACTH-Konzentration nach Nikotingabe an Hypophysenpräparate.

Viele Autoren sprechen sich für eine höhere Lokalisation der Nikotinwirkung aus und halten den Hypothalamus selbst oder seine Afferenzen für den primären Angriffspunkt von Nikotin (Matta et al., 1987; Weidenfeld et al., 1989; Caggiula et al., 1991). Matta et al. (1987) konnten durch eine Nikotinapplikation in verschiedene Regionen des dritten Ventrikels zeigen, dass die resultierenden ACTH-Werte je nach Region unterschiedlich waren: Die nikotin-induzierte Ausschüttung von ACTH war im unteren Ventrikelbereich, in dem die Kerne des Hypothalamus liegen, höher als nach einer Nikotingabe in die oberen Ventrikelbereiche. Die Autoren schlossen hieraus, dass der Wirkungsort von Nikotin am Hypothalamus selbst oder distal des unteren Teils des dritten Ventrikels liegt. Nach einer weiteren Untersuchung der Forschungsgruppe um S. Matta (Matta 
et al., 1990a) nehmen die Autoren an, dass das Nikotin nicht am Hypothalamus selbst wirkt, sondern über catecholaminerge Afferenzen des Hirnstamms eine Aktivierung der HHN-Achse bewirkt. Dies stellten sie fest, nachdem sie durch Gabe von Mecamylamin in den paraventrikulären Nukleus (PVN, für die Sekretion von CRH zuständig) einen Anstieg von CRH durch Nikotin nicht verhindern konnten. Wurde dieser Antagonist jedoch in den vierten Ventrikel gegeben, der durch die räumliche Nähe zum Hirnstamm gekennzeichnet ist, stellten Matta et al. (1990a) eine Blockade der Nikotinwirkung fest. In einer anderen Studie der Forschungsgruppe Matta et al. (1990b) wurde die Beteiligung der catecholaminergen Zellgruppen des Hirnstamms bezüglich der Nikotinwirkung auf die HHN-Achse untersucht. Die Autoren kamen zu dem Schluss, dass Nikotin im Hirnstamm adrenerge und noradrenerge Zellgruppen aktiviert und diese über ihre Afferenzen den Hypothalamus zur Sekretion von CRH anregen.

Desensibilisierung der akuten Nikotinwirkung Sharp und Beyer (1986) untersuchten nicht nur eine einmalige Nikotingabe, sondern analysierten die ACTH-Konzentration auch nach einer zweiten Gabe und konnten eine Desensibilisierung der Hormonantwort beobachten. Eine vorherige Nikotingabe reduzierte den durch eine zweite Nikotingabe resultierenden Anstieg des ACTHSpiegels. Diese geringere Hormonantwort hielt für circa sechs Stunden an. Die Autoren halten einen beschleunigten Abbau des Nikotins nach einer zweiten Gabe nicht für die Erklärung der beobachteten geringeren Hormonantwort. Ebenfalls schreiben sie dem negativen Feedback durch das nach der ersten Gabe ausgeschüttete Corticosteron als Erklärungsversuch keine große Bedeutung zu, da nach einer Adrenalektomie zwar höhere ACTH-Werten gemessen werden konnten, diese aber geringer waren als nach der ersten Nikotingabe.

Einige Veröffentlichungen erklären die Desensibilisierung im Sinne einer Veränderung auf Rezeptorebene. Hierauf wird im folgenden Abschnitt bei der Diskussion der Studien eingegangen, in denen Tieren chronisch Nikotin zugeführt wurde.

Akute Nikotingabe und Stressexposition Einige Studien dieses Abschnittes untersuchten die Hormone der HHN-Achse auch nach Nikotingabe und Stressexposition (Balfour et al., 1975; Altland et al., 1978; Lutfy et al., 2006; Cruz et al., 2008). Lediglich eine dieser Veröffentlichungen 
(Cruz et al., 2008) konnte keinen Einfluss einer Stressreaktion auf die Nikotinwirkung beobachten und resümiert somit, dass Nikotin keinen additiven Effekt bei der durch einen Stressor ausgelösten Corticosteron-Sekretion besitzt.

Lutfy et al. (2006) konnten bei einem ähnlichen Versuchsaufbau auch keine Unterschiede in der Corticosteron-Ausschüttung feststellen, nehmen aber an, dass die Hormonantwort auf Stress schon ohne eine Nikotingabe maximal war und durch Nikotin nicht weiter beeinflusst werden konnte. Der in dieser Studie gemessene ACTH-Anstieg über die Werte der Kontrollgruppe hinaus zeigt zum einen, dass Nikotin und Stress eine additive Wirkung auf die Ausschüttung von ACTH besitzen, und zum anderen, dass die Komponenten der HHN-Achse unterschiedlich auf Nikotin und Stress reagieren.

Die These der additive Wirkung bei der Aktivierung der HHN-Achse durch Nikotin und Stressexposition wird auch von anderen Studien zu diesem Thema unterstützt. So beobachteten Balfour et al. (1975) zwar eine ähnliche Stressreaktion bei Tieren, die Nikotin oder Kochsalz erhalten hatten, aber es zeigte sich, dass die Cortiocosteron-Werte der Nikotingruppe in der anschließenden Erholungsphase länger erhöht waren. Ebenso zeigte Altland et al. (1978), dass nach Laufarbeit und hoher Zigarettenrauch-Exposition die Cortiocosteron-Spiegel höher lagen als bei Laufarbeit allein. Diese Ergebnisse, ebenso wie die der Studien, die eine chronische Nikotingabe und Stress untersucht haben (s. u.), lassen zumindest auf eine partielle Beeinflussung der Stressreaktion durch Nikotin schließen.

Desensibilisierung der chronischen Nikotinwirkung In den Studien, in denen die Corticosteron- und/oder ACTH-Konzentration nach mehrtägiger Nikotingabe wieder auf Ausgangswerte sank, werden von den Autoren die verschiedensten Erklärungsmodelle diskutiert. Allgemein wird von Toleranz, Adaptation oder Desensibilisierung gesprochen (Benwell und Balfour, 1979; Caggiula et al., 1991; Pauly et al., 1992; Chen M et al., 2007; Cam und Bassett, 1984). Die Gründe für eine abnehmende hormonelle Antwort auf eine chronische Nikotingabe sind nicht geklärt, dennoch existieren hierzu einige Thesen.

Möglicherweise verändert eine mehrmalige Nikotingabe die Pharmakokinetik des Nikotins, so dass es schneller abgebaut wird. Diese Theorie wird von Cam und Bassett (1984) abgelehnt, da sich die 
Nikotinkonzentration im Blut der Versuchstiere nicht änderte. Auch Sharp und Beyer (1986) halten eine durch Nikotin induzierte Veränderung des eigenen Metabolismus für unwahrscheinlich. Sie beobachteten eine Desensibilisierung der Hormonantwort nach mehrmaliger akuter Nikotingabe, die für mindestens sechs Stunden anhielt. Chen M et al. (2007) hingegen halten einen erhöhten nikotin-induzierten Abbau von Nikotin für möglich. Sie verweisen auf eine Studie von Lee et al. (2006), in der das nikotinabbauende Enzym CYP2B6 im Gehirn von Affen durch Nikotin induziert und somit der Abbau desselbigen beschleunigt wurde.

Cam und Bassett (1984) nennen als mögliche Ursache der abnehmenden hormonellen Antwort auf Nikotin die negative Rückkopplung. Hierbei bewirkt ein durch Nikotin erhöhter Hormonspiegel des ACTHs oder des Corticosterons möglicherweise eine verminderte Aktivität der HHN-Achse. Dass diese jedoch auf andere Stressoren, beispielsweise psychologischer Natur, unverändert reagiert, erklären die Autoren mit der Hypothese, dass Nikotin und psychologischer Stress auf unterschiedlichen Signalwegen verarbeitet werden. Hierbei gehen sie auf der Ebene des Hypothalamus mit seinen CRH-freisetzenden Neuronen von verschiedenen Schwellenwerten bei der negativen Rückkopplung aus. Auf dem stressverarbeitenden Signalweg läge die Schwelle der negativen Rückkopplung höher als auf dem nikotinverarbeitende Signalweg. So würde die hormonelle Antwort auf Nikotin einer hohen negativen Rückkopplung unterliegen und bei chronischer Gabe schnell durch die steroidempfindlichen Neurone des Hypothalamus gehemmt. Die Antwort des Hypothalamus auf Stress hingegen würde über steroidresistente Neurone vermittelt und adaptiere somit nicht vollständig.

Einige Autoren halten die Entwicklung einer Desensibilisierung der Hormonantwort für das Resultat einer Veränderung auf Rezeptorebene. Wie unter 1.2.6 auf Seite 6 beschrieben, bewirkt ein chronischer Nikotinkonsum eine Vermehrung von nACh-Rezeptoren im ZNS. Caggiula et al. (1991) und Chen $\mathrm{M}$ et al. (2007) gehen davon aus, dass diese strukturellen Veränderungen zumindest einen Teil zur Toleranzentstehung bezüglich der HHN-Achse beitragen könnten. Da es durch eine chronische Nikotingabe nicht zu einer veränderten Noradrenalinausschüttung im PVN des Hypothalamus kam, schließen Chen M et al. (2007) die Beteiligung von nACh-Rezeptoren auf catecholaminerge Neuronen des Hirnstamms aus. Sie halten es vielmehr für möglich, dass die Desensibilisierung der Nikotinrezeptoren des posterioren paraventrikulären Thalamus für eine 
adaptierte Hormonantwort der HHN-Achse auf Nikotin sorgt, da diese Struktur schon bekannt dafür ist, auf wiederholten Stress mit einer Desensibilisierung der genannten Rezeptoren zu reagieren. Pauly et al. (1992) konnten in ihrer Studie den Zusammenhang zwischen der Toleranz und der Rezeptoranzahl hingegen nicht bestätigen.

Die Toleranz kann auch durch andere Mechanismen erklärt werden. So mag eine zentrale Inhibition, die Unfähigkeit der Hypophyse oder der Nebennieren, weiterhin auf Aktivatoren zu reagieren, oder ein erhöhter peripherer Umsatz von Corticosteroiden eine Rolle spielen. Gegen diese Thesen spricht allerdings, dass die Tiere, die chronisch Nikotin erhielten, eine adäquate hormonelle Reaktion auf einen neuen Stressor zeigten und somit die Funktion der HHN-Achse nicht generell beeinflusst zu sein scheint (Cam und Bassett, 1984).

Nikotinentzug Betrachtet man die vier Studien, die auch einen Entzug untersucht haben (Benwell und Balfour, 1979; Semba et al., 2004; Andersson et al., 1989; Pauly et al., 1992), lässt sich kein Trend für den Hormonkonzentrationsverlauf feststellen. Andersson et al. (1989) wollen mit ihren Untersuchungsergebnissen eine Erklärung für die Entstehung von Entzugserscheinungen (insbesondere psychologischer Natur) gefunden haben. Sie stellten am zweiten Entzugstag nach einer zehntägigen Nikotinexposition eine verminderte Corticosteron-Konzentration bei unveränderter ACTH-Konzentration im Blut von Ratten fest. Dieses hormonelle Ungleichgewicht, zustandekommend möglicherweise durch eine Desensibilisierung von ACTH-Rezeptoren der Nebennierenrinde, soll einen Einfluss auf die Verhaltenspychologie im Entzug haben.

Chronische Nikotingabe, Nikotinentzug und Stressexposition Auch die Studien, die eine chronische Nikotingabe oder einen Nikotinentzug untersuchten, haben sich mit einer möglicherweise durch Nikotin verursachten veränderten Reaktion der HHN-Achse auf Stress auseinandergesetzt (Faraday et al., 2005; Morse, 1989; Chen H et al., 2008; Semba et al., 2004). Ähnlich wie bei den Studien, in denen eine akute Nikotingabe thematisiert wurde, wurden auch hier unterschiedliche Ergebnisse erhalten. Chen H et al. (2008) konnten keine Modulation der hormonellen Antwort auf Immobilisationsstress durch eine chronische Nikotingabe feststellen, jedoch bei der Verabreichung von geringen Elektroschocks. Demnach scheint die Qualität des Stressors eine bedeutende 
Rolle bei der Beeinflussung der HHN-Achse durch Nikotin zu spielen. Dass hierbei die Größe des Schmerzreizes von Bedeutung ist, zeigt sich daran, dass man diese Beeinflussung nur bei einer geringen Stromstärke beobachten konnte.

Morse (1989) und Faraday et al. (2005) konnten ebenfalls eine veränderte Hormonantwort bei der Konstellation Nikotin und Stress im Gegensatz zu Nikotin oder Stress allein feststellen. Diese fiel bei Morse et al. höher aus und unterstützt somit die These der additiven Wirkung von Nikotin auf die Stressreaktion. In der Arbeit von Faraday et al. (2005), in der eine geschlechtliche Differenzierung vorgenommen wurde, wurde dies nur für weibliche Ratten gezeigt. Die Unterschiede zwischen den Geschlechtern führen die Autoren nicht auf den weiblichen Hormonzyklus zurück, sie schließen die hormonelle Komponente bei der Verarbeitung von Stress und Nikotin jedoch nicht gänzlich aus.

Interessanterweise konnten bei den chronisch mit Nikotin versorgten Tieren geringere Corticosteron-Werte nach den Stresstests gemessen werden als bei der Kontrollgruppe, die Kochsalz erhalten hatte. Welche Umstände an dieser Beeinflussung der Stressreaktion beteiligt sind, ist nicht geklärt. Möglicherweise induziert eine chronische Nikotingabe eine geringere Sensitivität der HHN-Achse.

Semba et al. (2004) stellten nach einer Entzugszeit von zwei Tagen ebenfalls eine geringere Sensitivität der HHN-Achse bei der Antwort auf einen Stressor fest und halten diesen Umstand für das Resultat desensibilisierter nACh-Rezeptoren. Zudem sprechen die Autoren sich für einen Zusammenhang zwischen einer geringeren Sensitivität der HHN-Achse und einer depressiven Stimmung während des Entzugs aus.

\section{Nichtraucher}

Bis auf eine Studie (Gossain et al., 1986) konnte in allen Veröffentlichungen, in denen Nichtrauchern Nikotin verabreicht wurde, eine Erhöhung der Cortisol- und ACTH-Konzentration gemessen werden. Für die Mechanismen, die hierbei eine Rolle spielen könnten, sei auf den Abschnitt der tierexperimentellen Arbeiten verwiesen. 
Gossain et al. (1986) stellten als einzige Veröffentlichung in diesem Abschnitt fest, dass sich die Blut-Cortisolkonzentration bei Nichtrauchern nach Nikotingabe nicht änderte. Bei dem Vergleich von Nichtrauchern mit Rauchern konnten sie keine Unterschiede in den basal gemessenen Hormonwerten feststellen, jedoch zeigte sich bei der Gruppe der Raucher ein Anstieg des Cortisolspiegels nach dem Rauchen von zwei Zigaretten. Diesen Umstand diskutieren die Autoren als einen Ausdruck einer erhöhten Sensitivität der HHN-Achse bei Rauchern und somit reiht sich diese These in die Reihe von Spekulationen über die Beeinflussung der Ansprechbarkeit der HHN-Achse durch Nikotin ein.

Perkins et al. (2008) konnten genetische Unterschiede bei der hormonellen Antwort auf Nikotin entdecken und stellten fest, dass nur die Träger des 7-Repeat-Allels des D4-Rezeptors eine Erhöhung der Cortisolkonzentration nach Nikotin zeigten. Bei dieser Probandengruppe sprachen auch weitere Untersuchungsergebnisse für eine aversive Reaktion auf Nikotin. Menschen mit dieser genetischen Konstellation sind nach Meinung der Autoren möglicherweise weniger für die Entwicklung einer Nikotinsucht prädestiniert, da die initiale Sensitivität für Nikotin erhöht zu sein scheint. Da allerdings bei Nagetieren gezeigt wurde, dass Tiere, die eine hohe Sensitivität für Nikotin besitzen, schnell eine starke Toleranz für diese negativen Effekte entwickeln, ist das Risiko für eine Nikotinabhängigkeit bei Menschen mit diesem Genotyp möglicherweise erhöht.

\section{Raucher}

Bei der Betrachtung der Studien, die einen Nikotinentzug von Rauchern untersucht haben, lässt sich eine deutliche Abhängigkeit der Studienergebnisse von der Entzugszeit feststellen. Die Studien, die die ersten 24 Stunden eines Nikotinentzugs untersuchten, kamen zu keinem einheitlichen Ergebnis. Im Zeitraum weniger Entzugstage hingegen ergab sich ein deutlicheres Bild, denn fast alle Veröffentlichungen beobachteten in dieser Phase keine Veränderungen der Cortisolkonzentration. Die Langzeitstudien sprechen sich einheitlich dafür aus, dass es während einer Entzugszeit von mehreren Wochen zu einem Absinken der Cortisolkonzentration kommt.

Unterschiede zwischen Rauchern und Nichtrauchern bezüglich der HHN-Achse Inwieweit ein akuter oder chronischer Nikotinkonsum auf die HHN-Achse bei Rauchern wirkt, wird 
z. T. kontrovers diskutiert, aber eine Vielzahl von Autoren unterstützt folgende Theorie: Betrachtet man die akuten hormonellen Veränderungen, die durch das Rauchen bewirkt werden, wurde in vielen Studien gezeigt, dass nur ein hoher Nikotinkonsum in der Lage ist, die Cortisolkonzentration signifikant zu erhöhen (Gilbert et al., 1992; Seyler et al., 1984; Wilkins et al., 1982), da sich die HHN-Achse durch den anhaltenden Nikotinkonsum möglicherweise in ihrer Reaktivität verändert hat (Toleranzentstehung) oder generell nur hohe Dosen eine Aktivierung der HHN-Achse bewirken (Dosisabhängigkeit). In Hinblick auf die durchschnittlichen Hormonkonzentrationen, die z. B. über den Tag hinweg bei Rauchern vorliegen, postulieren viele Autoren, dass diese im Gegensatz zu Nichtrauchern erhöht sind (Badrick et al., 2007; Baron et al., 1995; Kirschbaum et al., 1992). Rohleder und Kirschbaum (2006) und Kirschbaum et al. (1992) nehmen diesbezüglich an, dass diese Erhöhung nicht durch eine generell erhöhte basale Aktivität der HHN-Achse bei Rauchern zustande kommt, sondern durch die Summe der Hormonkonzentrationsspitzen, verursacht durch die gerauchten Zigaretten.

Dennoch scheint ein chronischer Nikotinkonsum die Reaktivität der HHN-Achse bei Rauchern zu beeinflussen, insbesondere als Antwort auf psychischen Stress. Dies konnte in den Studien aus dem Arbeitskreis um C. Kirschbaum festgestellt werden. Es zeigte sich in mehreren Versuchsreihen, dass Raucher eine geringere hormonelle Antwort der HHN-Achse auf Stress aufwiesen als Nichtraucher (Kirschbaum et al., 1993; Rohleder und Kirschbaum, 2006). Eine Erklärung für diese Desensibilisierung finden die Autoren nicht, halten aber eine Veränderung auf Ebene der Glucocorticoidrezeptoren für möglich. Wurde die Reaktivität der HHN-Achse mithilfe des DSTs gemessen, konnten Hughes et al. (1988), Frederick et al. (1998) und Pomerleau et al. (2004) hingegen keine Auffälligkeiten entdecken.

An dieser Stelle sei auf die tierexperimentellen Arbeiten verwiesen, in denen ebenfalls eine veränderte Stressreaktion nach Nikotingabe auffiel. Diese Ergebnisse decken sich z. T. mit denen der Raucher. Es wurde für die akute Situation gezeigt, dass Nikotin und Stressexposition gemeinsam eine additive Wirkung auf die Antwort der HHN-Achse besitzen. Zum anderen wurde eine geringere generelle Reaktivität der HHN-Achse auf Stress bei Mensch und Tier beobachtet.

Akuter Nikotinentzug Cohen et al. (2004) begründen ihre Beobachtung (ein Absinken der 
Speichel-Cortisolkonzentration im vierstündigen Entzug) mit der Hypothese, dass ein chronischer Nikotinkonsum mit funktionellen und strukturellen Änderungen im ZNS einhergeht und diese den hormonellen Konzentrationsverlauf im Entzug erklären könnten. Sie sprechen in diesem Zusammenhang von einem Rebound der Antwort der HHN-Achse, also von einem der Nikotinwirkung entgegengesetzten Effekt.

Hughes et al. (1988) erklären den Anstieg der Cortisolkonzentration innerhalb eines 24-stündigen Entzugs in ihrer Arbeit damit, dass die Probanden, die im Gegensatz zu anderen Veröffentlichungen wirklich gewillt waren, mit dem Rauchen aufzuhören, dies als Stress empfanden und somit die HHN-Achse aktiviert wurde.

Die Ergebnisse der Mehrzahl der Studien, die einen Entzug über mehrere Tage verfolgten, sprechen dafür, dass es in dieser Zeit zu keinen großen Änderungen der Hormonspiegel kommt. Teneggi et al. (2002) stellten am ersten Entzugstag eine geringere Cortisolkonzentration im Urin der abstinenten Raucher fest. Im Blut hingegen ließ sich dies nicht nachweisen. Unter Einsatz von Nikotinpflastern konnte dieser Abfall ebenfalls nicht beobachtet werden. Pomerleau et al. (2000) konnten hingegen zeigen, dass die Cortisolkonzentration während eines elftägigen Entzugs abfiel. Auch bei Rasmusson et al. (2006) wurde ein Trend für einen Cortisolabfall innerhalb eines achttägigen Entzugs bei den nach 15 Tagen noch abstinenten Probanden gefunden. Für die Interpretation dieser Ergebnisse wird auf den folgenden Abschnitt verwiesen, in dem ein längerfristiger Nikotinentzug diskutiert wird.

Chronischer Nikotinentzug Die Studien, die einen Entzug über mehrere Wochen untersucht haben, kommen einheitlich zu dem Ergebnis, dass die Cortisolkonzentration im Blut und/oder Speichel bei abstinenten Probanden sinkt. Die Autoren halten diesen Umstand für den Ausdruck des Wegfalls der stimulierenden Wirkung des Nikotins auf die Hormone der HHN-Achse. Ussher et al. (2006) beispielsweise sprechen von einem Rebound der Hormonkonzentration. Die bei Rauchern chronisch erhöhten Cortisolspiegel bewirken im Organismus des Rauchers möglicherweise die Entwicklung gegensteuernder Mechanismen, um einen Hypercortisolismus zu verhindern. Fällt nun die stimulierende Wirkung des Nikotins im Entzug weg, kommt es zu dem beobachteten starken Cortisolabfall, wie es in der Untersuchung bei Ussher et al. (2006) der Fall war. Hier zeigte 
sich auch ein im Verlauf des Entzugs wieder leicht ansteigender Cortisolspiegel - dies könnte somit für eine Regeneration des Hormonsystems sprechen.

Rückfallwahrscheinlichkeit Die Studien, die einen chronischen Nikotinentzug untersuchten, widmenten sich, wie auch einige der Studien, die einen akuten Entzug untersuchten, den Konsequenzen eines starken Cortisolabfalls und den Zusammenhängen der Hormonveränderungen mit der Entzugssymptomatik und der Aussicht auf einen erfolgreichen Entzug.

Frederick et al. (1998) konnten eine Korrelation der Höhe des Cortisolabfalls mit den auftretenden Entzugserscheinungen im Entzug feststellen. Je stärker die Cortisolkonzentration sank, desto höher waren die Entzugserscheinungen und damit die Wahrscheinlichkeit eines Rückfalls (die Vorhersage eines Rückfalls war nicht signifikant). Diese Beobachtung machten auch Ussher et al. (2006). Auch sie entdeckten den Zusammenhang zwischen einem Cortisolabfall im Entzug und einer erhöhten Rückfallwahrscheinlichkeit (ebenfalls nicht signifikant). Zudem stellten sie eine Korrelation des Cortisolspiegels im Entzug und den Entzugserscheinungen und dem Verlangen nach Zigaretten fest. Je niedriger die Cortisolwerte waren, desto stärker waren die Entzugssymptome und das Verlangen. In der Studie von al'Absi et al. (2004) konnte zudem gezeigt werden, dass ehemalige Raucher, die einen stärkeren morgendlichen Abfall der Cortisolkonzentration aufwiesen, ein erhöhtes Risiko für einen Rückfall hatten.

Dass insbesondere starke Raucher ein erhöhtes Risiko für einen Rückfall hatten, wurde in mehreren Veröffentlichungen gezeigt (z. B. in Ussher et al., 2006; Dušková et al., 2010). Ussher et al. (2006) stellten eine Verbindung zwischen der Cortisolkonzentration im Entzug und dem ehemaligen Nikotinkonsum her. War dieser besonders stark, zeigte sich ein stärkerer Hormonabfall als bei weniger starken Rauchern. Die Autoren nehmen an, dass ein hoher Nikotinkonsum höhere Cortisolspiegel verursacht und dieser somit im Entzug einen besonders starken Abfall zeigt, was die oben genannten Konsequenzen eines erhöhten Rückfallrisikos mit sich bringt. Auch Cohen et al. (2004) beobachteten eine positive Korrelation der Cortisolkonzentration vor dem Entzug mit den Entzugserscheinungen und dem Verlangen nach Zigaretten im Entzug.

Durch welche Mechanismen ein niedriger Cortisolspiegel im Entzug die typische Entzugssymptomatik auslöst und wie dies mit dem Risiko eines Rückfalls verbunden ist, ist nicht gänzlich geklärt. 
Rasmusson et al. (2006) halten es diesbezüglich für möglich, dass bei Rauchern im Entzug eine Art Restauration der adaptierten Funktion der HHN-Achse stattfindet, die sich in einer veränderten Reaktivität derselben widerspiegelt. Möglicherweise reagierten die Probanden, die einen Rückfall erlitten, auf diese Veränderung sensibler und erfahren z. B. auf Stress eine stärkere Aktivierung der HHN-Achse. Den Zusammenhang zwischen Stress und Rückfallwahrscheinlichkeit nehmen auch al'Absi et al. (2002) an und halten es für möglich, dass ein höherer Stresslevel vermehrt Entzugserscheinungen verursacht und somit das Rückfallrisiko erhöht ist.

Im Gegensatz hierzu steht eine andere Theorie: Frederick et al. (1998) nehmen an, dass gerade die ehemaligen Raucher, die eine erhöhte Reaktivität der HHN-Achse auf Entzugsstress zeigen, eher abstinent bleiben. Möglicherweise wirkt bei diesen Menschen der durch Stress erhöhte Cortisolspiegel dem durch den Entzug ausgelösten Cortisolabfall und damit dem Rebound entgegen, was zu einer Abnahme des Stresses und einer geringeren Rückfallwahrscheinlichkeit führt. Auch Ussher et al. (2006) und al'Absi et al. $(2003,2005)$ postulieren, dass die bei Rauchern beobachtete geringe Reaktivität der HHN-Achse Stress verursacht und mit einem erhöhten Rückfallrisiko verbunden ist.

Doch nicht alle Studien können einen Zusammenhang zwischen Cortisolkonzentration und Entzugserscheinungen oder der Rückfallwahrscheinlichkeit nachweisen. Ceballos und al'Absi (2006) und Hughes et al. (1988) finden keine Korrelationen.

Zusammenfassend scheint es im Falle eines Entzugs und dem einhergehenden Cortisolabfall zu einer endokrinen Dysbalance zu kommen, die psychischen und physischen Stress auslöst und somit die Rückfallwahrscheinlichkeit erhöht. Inwieweit sich die messbaren Hormonveränderungen, die Entzugssymptome und die Stressantwort gegenseitig bedingen oder welcher Umstand welche Konsequenzen auslöst und was das für die Rückfallwahrscheinlichkeit bedeutet, muss noch geklärt werden. Auch die Beteiligung anderer Transmittersysteme (z. B. das der Catecholamine) darf hierbei nicht außer Acht gelassen werden. Auch die individuelle Ausgangssituation scheint von großer Bedeutung zu sein. Wichtige Faktoren hierbei sind der Grad der Nikotinabhängigkeit, die individuelle Größe der durch das Rauchen entstandenen Toleranz, die Sensitivität der Stressantwort und die psychologischen Coping-Mechanismen. 


\subsection{Schlussfolgerungen und Ausblick}

Im Folgenden sollen die konkreten Fragen aus 1.7 auf Seite 27 beantwortet und Schlussfolgerungen gezogen werden. Der Frage nach den Lücken in der Nikotinforschung soll im Abschnitt „Ausblick“ nachgegangen werden.

\section{Schlussfolgerungen}

\section{Auswirkungen einer Nikotingabe und eines Nikotinentzugs und Vergleiche zwischen} den Probandengruppen Aus den Studien, die durch die systematische Literaturrecherche ausfindig gemacht wurden, geht hervor, dass Nikotin entscheidend in zentralnervöse Systeme und Regulationen eingreift. Mehrheitlich wird davon ausgegangen, dass die Nikotinwirkungen über zentrale nikotinische Acetylcholin-Rezeptoren vermittelt werden - diese Erkenntnis wurde aus Untersuchungen mit Mecamylamin, einem Antagonist der genannten Rezeptoren, gewonnen.

Durch die Bindung des Nikotins an die Nikotinrezeptoren kommt es zu einer vermehrten Freisetzung der verschiedensten Neurotransmitter, welche Teil der Steuerung des Schlafs, der HHN-Achse oder des Belohnungssystems sind. Dementsprechend können die normalen Funktionen und Abläufe in den verschiedenen Bereichen des ZNS durch Nikotin verändert werden. Ausdruck findet dies in den Untersuchungen an nikotinnaiven Lebewesen, in denen regelmäßig Veränderungen im Schlaf und endokrinen Systemen beobachtet werden konnten. In den tierexperimentellen Studien zeigte sich eine dosisabhängige Wirkung von Nikotin auf den Schlaf, wobei geringe Nikotindosen den REM-Schlaf erhöhten, höhere Dosen jedoch die Gesamtschlafzeit und dementsprechend den REM- und NREM-Schlaf verringerten. Nikotin verminderte bei gesunden Nichtrauchern den REM-Schlaf, bei depressiven hingegen erhöhte sich dieser polysomnografische Parameter. Zudem besserten sich die Symptome der Depression. Lediglich eine Studie untersuchte die Wirkung von Nikotin auf den Schlaf von Nichtrauchern über mehrere Monate (Haro und Drucker-Colín, 2004) und diese beschränkte sich auf depressive Teilnehmer. Es zeigte sich, dass Nikotin bei depressiven Probanden einen erhöhten Anteil an Tiefschlaf, eine verlängerte Gesamtschlafzeit und REM-Latenz und eine Besserung der Depressionen bewirkte. Diesbezüglich ist anzumerken, dass sich die meisten Antidepressiva dadurch auszeichnen, den REM-Schlaf zu supprimieren (Mayers und Baldwin, 
2005). In der Langzeitstudie spiegelte sich die Suppression des REM-Schlafs in der verlängerten REM-Latenz wider. Die Konzentration der Hormone der HHN-Achse stieg nach einer Nikotingabe in tierexperimentellen Untersuchungen oder an Nichtraucher mehrheitlich an.

Einige Studien, die eine längerfristige Nikotingabe untersuchten, liefern Hinweise darauf, dass Nikotin nicht nur akut in die Regulation des Schlafs und des Endokrinums eingreift, sondern auch auf längere Sicht Veränderungen im ZNS hervorruft. Beispielsweise sei hier die Ausbildung einer Toleranz gegenüber der Nikotinwirkung erwähnt, die nach mehrmaliger Gabe in den Studien auftrat, die sich mit der HHN-Achse auseinandergesetzt haben. Auch tierexperimentelle Untersuchungen zeigten eine veränderte Dopaminausschüttung durch chronische Nikotingaben.

Allem Anschein nach ist Nikotin in der Lage, die Funktionen des ZNS längerfristig zu beeinflussen. Die strukturellen Korrelate hierfür sind Gegenstand aktueller Forschung und waren - was die Studien über das dopaminerge System betrifft - mitunter Teil der Fragestellung dieser Arbeiten, in denen Raucher und Nichtraucher miteinander verglichen wurden. Hieraus, wie auch aus anderen Untersuchungen, die sich z. B. mit der Up-Regulation von Nikotinrezeptoren durch Nikotin beschäftigten, wird deutlich, dass Nikotin neben der Funktion auch die Struktur des ZNS verändern kann, wie es schon für andere suchterzeugende Substanzen gezeigt wurde (Volkow et al., 1996, 1997; Wang et al., 1997). So werden diese chronischen Veränderungen als neurobiologische Korrelate der Abhängigkeit diskutiert.

Die tierexperimentellen Versuche oder die Studien mit Nichtrauchern können Hinweise darauf liefern, was ein Nikotinkonsum bewirken kann. Dennoch ist diesbezüglich anzumerken, dass die möglicherweise im ZNS von Rauchern vorliegenden strukturellen und funktionellen Veränderungen völlig anderer Art sein können. Ein jahrelanger Nikotinkonsum kann nicht durch eine kurzfristige Nikotingabe simuliert werden. Ein Vergleich zwischen Rauchern und Nichtrauchern nach Nikotingabe ist demnach sehr kritisch zu bewerten.

Dass sich ein chronischer Nikotinkonsum auf die Funktion und Struktur des ZNS auswirkt, zeigt der Blick auf den Zustand des Nikotinentzugs. Auch hierbei lassen sich Veränderungen polysomnografischer und neuroendokriner Parameter feststellen. Somit erfährt ein durch eine chronische Nikotingabe verändertes ZNS ein Ausbleiben der gewohnten Nikotindosis mit all ihren Wirkungen. Die polysomnografischen Untersuchungen zeigten, dass in den ersten Entzugstagen keine 
wesentlichen Veränderungen des REM- oder NREM-Schlafs stattfanden, jedoch eine erhöhte Fragmentierung des Schlafs beobachtet werden konnte. Der Einsatz von Nikotinersatztherapien konnte insbesondere bei männlichen Probanden den Schlaf verbessern (weniger Schlaffragmentierung). In der einzigen Langzeitstudie (Moreno-Coutiño et al., 2007) wurde eine Erhöhung des REM-Schlafs und eine Verminderung der Gesamtschlafzeit im Nikotinentzug beobachtet. Die Konzentration der Hormone der HHN-Achse blieb in den ersten Entzugstagen gleich, nahm jedoch im Langzeitentzug ab. Die Studien, die sich mit dopaminergen Parametern auseinandersetzten, kamen zu dem Ergebnis, dass in den ersten 12 bis 24 Stunden des Entzugs keine Veränderungen auftraten. Im Langzeitentzug, welcher in zwei Studien untersucht wurde, konnte eine Zunahme des Bindungspotentials an D1-Rezeptoren und eine Abnahme der Urin-Dopaminkonzentration festgestellt werden.

Bezüglich des dopaminergen Systems oder der HHN-Achse vertreten einige Autoren die Meinung, dass die bei chronischen Rauchern vorliegenden Veränderungen reversibel sind und im Entzug eine Art Restauration erfahren, sobald Nikotin mit seinen zahlreichen Wirkungen wegfällt. Inwieweit sich die gemessenen Parameter wieder den Werten von Nichtrauchern angleichen, ist aus den Studien nicht ersichtlich.

Ein Vergleich zwischen Nichtrauchern und Rauchern nach Nikotingabe erbrachte in einer Forschungsgruppe, dass sich die Cortisolkonzentration nur bei den Nichtrauchern erhöhte (Sellini et al., 1989a,b,c). Gossain et al. (1986) stellten hingegen fest, dass sich die Blut-Cortisolkonzentration bei Nichtrauchern nach Tabakkonsum nicht änderte, jedoch bei Rauchern erhöht war. Die Autoren sprechen in diesem Zusammenhang von einer erhöhten Sensitivität der HHN-Achse bei Rauchern.

Ein wichtiger Punkt bei der Betrachtung der gefundenen Literatur ist die eingeschränkte Vergleichbarkeit. Insbesondere sind Kurz- und Langzeitentzugsstudien schwer miteinander zu vergleichen, da unterschiedliche Voraussetzungen bestehen, was möglicherweise einen Einfluss auf die Ergebnisse haben kann. Sicherlich ist die Motivation des Rauchers von großer Bedeutung, die maßgeblich die gemessenen Parameter beeinflussen kann. In vielen Studien, die nur eine kurze Entzugszeit begleiteten, hatten die Raucher nicht vor, langfristig abstinent zu sein, und verzichteten nur für die kurze Studienzeit auf das Rauchen. In Studien, die eine längere Abstinenz beobachteten, ist dies 
meist nicht der Fall, die Raucher hatten wirklich vor, mit dem Rauchen aufzuhören. Die intentionelle Grundvoraussetzung ist demnach eine völlig andere und kann sich z. B. über psychologischen Stress auf die Funktion der HHN-Achse auswirken. Ein Raucher, der endgültig mit dem Rauchen aufhören will, hat demnach möglicherweise mehr Stress, der sich auf die HHN-Achse und andere Bereiche wie beispielsweise die Schlafregulation, auswirken kann.

Prädiktoren eines Rückfalls Um Faktoren zu finden, die einen Rückfall begünstigen oder einen Entzug erfolgreich verlaufen lassen, benötigt man Studien, in denen ein Vergleich von Messwerten von abstinenten und rückfälligen Probanden möglich ist. Im direkten Vergleich könnten Unterschiede oder charakteristische Verläufe der gemessenen Parameter erkennbar sein. Wichtig hierbei sind Unterschiede vor dem Entzug, der Verlauf des Entzugs und die Auswirkungen eines Rückfalls auf die Parameter.

Teilt man den Entzug in verschiedene Abschnitte ein (Beginn, Verlauf, Rückfall oder Abstinenz), könnte es in jeder dieser Phasen Faktoren geben, die den Entzug beeinflussen. Die individuelle Ausgangssituation des Rauchers vor dem Entzug scheint von großer Wichtigkeit zu sein. Die Dauer und Intensität des Rauchens mag hierbei eine Rolle spielen, da der chronische Nikotinkonsum möglicherweise zu Veränderungen in der Funktion und/oder der Struktur des ZNS des Rauchers geführt hat, die einen erfolgreichen Entzug, beispielsweise durch verstärkte Entzugserscheinungen, erschweren. Der Verlauf des Entzugs bezüglich des Schlafs oder des Endokrinums scheint ebenfalls eine große Bedeutung für die Wahrscheinlichkeit eines erfolgreichen Entzugs zu haben. Dies ging zumindest aus einigen Veröffentlichungen hervor, die sich mit der HHN-Achse auseinandersetzten (z. B. war ein großer Abfall der Cortisolkonzentration mit starken Entzugserscheinungen korreliert, die zu einem Rückfall führen können). Betrachtet man die Auswirkungen eines erneuten Nikotinkonsums nach einer gewissen Entzugszeit, spielen diese möglicherweise auch für den weiteren Verlauf der Nikotinabhängigkeit eine große Rolle. Möglicherweise reagiert ein Mensch, dessen Belohnungssystem an den Nikotinkonsum adaptiert war und der sich im Entzug in einem hypodopaminergen Zustand befindet, sehr empfindlich auf eine erneute Nikotingabe, was sich in einer starken Aktivierung des Belohnungssystems widerspiegeln kann. Der Rückfall wird demnach positiv verstärkt und eine weitere Abstinenz erschwert. 
In den meisten Studien ist ein Vergleich abstinenter und rückfälliger Probanden nicht möglich, da die Probanden, sobald sie rückfällig wurden, aus den weiteren Untersuchungen ausgeschlossen wurden und die entscheidenden Daten fehlen. Zudem wird in vielen Untersuchungen nur der akute Entzug von wenigen Stunden untersucht.

Betrachtet man die Studien, die sich mit dopaminergen Parametern auseinandergesetzt haben, findet sich lediglich eine Veröffentlichung (Yasuno et al., 2007), in der abstinente und rückfällige Probanden miteinander verglichen wurden. Es kam heraus, dass das Bindungspotential an D1Rezeptoren im Striatum vor dem Entzug keinen prädestinierenden Faktor einer erfolgreichen Abstinenz oder eines Rückfalls darstellt.

Bezüglich der HHN-Achse sind einige Forschungsgruppen der Frage nachgegangen, welche Faktoren einen Rückfall vorhersagen können. Es konnte eine Korrelation zwischen der Cortisolkonzentration und der Entzugssymptomatik ausfindig gemacht werden. Je stärker der Cortisolabfall im Entzug, desto höher waren die Entzugserscheinungen und desto wahrscheinlicher ein Rückfall. Demnach kann die Cortisolkonzentration im Verlauf des Entzugs anscheinend als Prädiktor für einen Rückfall oder einen erfolgreichen Entzug angesehen werden.

\section{Nutzen der Beobachtung des Schlafs, des dopaminergen Systems oder der HHN-Achse}

im Entzug Um den Verlauf eines Entzugs beurteilen zu können und mögliche Prädiktoren zu erkennen, ist die Wahl der Messparameter von entscheidender Bedeutung. Nur wenn ein Entzug durch ein objektivierbares Messverfahren dargestellt werden kann, können aus den Messergebnissen weitere Schlüsse gezogen werden. Eine Fragestellung der vorliegenden Arbeit ist demnach, ob es sinnvoll ist, die ausgewählten Parameter während eines Entzugs zu beobachten, um beispielsweise Prädiktoren zu identifizieren. Um diese Frage zu beantworten, müssen möglichst viele Studien mit ähnlichen Methoden vorliegen, in denen signifikante Veränderungen der Parameter im Entzug gemessen werden konnten und diese Veränderungen auf den Entzug zurückzuführen sind. Durch die geringe Studienzahl in einigen Themengebieten und die unzureichende Vergleichbarkeit der Studien kann die Frage nach dem Nutzen der Beobachtung des Schlafs und neuroendokriner Parameter nicht abschließend beantwortet werden. Es wird jedoch im Folgenden versucht, den Nutzen der untersuchten Messverfahren - wenn auch stellenweise theoretisch - einzuschätzen. 
Die Polysomnografie liefert mit ihren zahlreichen Ableitungen eine Vielzahl an Messergebnissen, die Einblicke in die unterschiedlichsten Körperfunktionen bietet. So können über die Verteilung der Schlafstadien indirekt Rückschlüsse auf dominierende Transmittersysteme gezogen (siehe 1.3.3 auf Seite 18) oder über die Ableitung der Beinbewegungen möglicherweise Funktionen des dopaminergen Systems beobachtet werden. Durch die Literaturrecherche konnten einige Studien gefunden werden, die signifikante Änderungen polysomnografischer Parameter im Nikotinentzug identifizierten. Beispielsweise sei hier die erhöhte Fragmentierung des Schlafs während der ersten Entzugstage genannt. Die Polysomnografie ist demnach in der Lage, objektive Messdaten zu liefern, die einen Entzug widerspiegeln.

Die Beobachtung des dopaminergen Systems im Entzug ist - zumindest theoretisch betrachtet sinnvoll, da Nikotin in der Lage ist, das dopaminerge System kurz- und längerfristig zu beeinflussen. Dass es im Entzug zu Veränderungen im dopaminergen System kommt, wurde zumindest in einer Studie gezeigt (Yasuno et al., 2007). Es wurde eine Zunahme des Bindungspotentials an D1-Rezeptoren in der PET beobachtet. Mit den geeigneten Messverfahren sind demnach Veränderungen darstellbar. Die vorliegende Übersichtsarbeit konnte allerdings nur sehr wenige Studien ausfindig machen, die die Folgen eines Entzugs für dopaminerge Parameter untersuchten. Aus diesen spärlichen Ergebnissen lässt sich nicht vollends klären, ob die Beobachtung des dopaminergen Systems, beispielsweise mithilfe der PET, sinnvoll ist, um den Verlauf eines Entzugs beurteilen zu können.

Ein Großteil der Studien, die einen Nikotinentzug untersucht haben, beschäftigte sich mit der HHN-Achse. Es konnten insbesondere im Langzeitentzug Veränderungen in der Konzentration von Hormonen der Stressachse aufgedeckt werden (ein signifikanter Konzentrationsabfall) sowie Korrelationen zwischen den Hormonkonzentrationen und subjektiven Entzugssymptomen (z. B. eine negative Korrelation zwischen der Cortisolkonzentration und Entzugserscheinungen im Entzug). Diese Studienergebnisse sprechen dafür, dass eine Beobachtung der HHN-Achse im Nikotinentzug gerechtfertigt ist.

Korrelationen von objektiven und subjektiven Messergebnissen Idealerweise können subjektiv empfundene Entzugserscheinungen durch objektive Messverfahren nachvollzogen wer- 
den. Korrelationen von objektiven und subjektiven Messwerten können zumindest einen Hinweis darauf geben, wodurch beispielsweise bestimmte Entzugssymptome ausgelöst werden. In einigen Studien wurden solche Korrelationen gefunden. Beispielsweise stellten Ussher et al. (2006) fest, dass eine niedrige Cortisolkonzentration am ersten Entzugstag mit dem Verlangen nach Zigaretten am dritten und vierten Entzugstag assoziiert war. Auch Yasuno et al. (2007) konnten eine Korrelation von dem Verlangen nach Zigaretten mit einem objektivierbaren Parameter ausmachen: Je niedriger das Bindungspotential an D1-Rezeptoren im Striatum, desto höher war das Verlangen der Probanden nach Zigaretten. Interessanterweise sind die neurobiologischen Korrelate der subjektiven Entzugserscheinungen, wie z. B. das Craving, in der Nikotinabhängigkeit wenig erforscht. Psychische Symptome können den ehemaligen Raucher auch noch nach jahrelanger Abstinenz belasten - objektivierbare Befunde gibt es allerdings nicht. In diesem Punkt ist die Forschung, die sich mit der Alkoholabhängigkeit auseinandersetzt, einige Schritte weiter (siehe hierzu Breese et al., 2011).

\section{Ausblick}

Trotz der vielen gefundenen Studien existieren große Lücken in der Nikotinforschung. Vieles ist bis heute gänzlich unerforscht. Beispielsweise konnte keine Veröffentlichung ausfindig gemacht werden, die sich den Konsequenzen einer Nikotingabe auf das dopaminerge System von Nichtrauchern widmet. Ebenso konnten keine Untersuchungen gefunden werden, die Menschen untersuchten, die nur gelegentlich rauchen. Dabei wäre diese Probandengruppe von großem Interesse, was beispielsweise die Unterschiede zu Rauchern betrifft.

Auffällig ist zudem, dass sich nach aktuellem Kenntnisstand bisher nur drei Forschungsgruppen mit den Folgen einer Nikotingabe für den Schlaf von Nichtrauchern auseinandergesetzt haben. Es gibt keine Studie, die die Langzeitwirkungen von Nikotin auf den Schlaf gesunder Nichtraucher untersucht. Ohnehin fällt auf, dass sich bisher wenige Forschungsgruppen mit den Auswirkungen einer Nikotingabe oder eines Nikotinentzugs auf polysomnografische oder dopaminerge Parameter beschäftigt haben. Auch ein Vergleich zwischen Nichtrauchern nach Nikotingabe mit Gelegenheitsrauchern oder Rauchern bezüglich des Schlafs oder des dopaminergen Systems fehlen, ebenso wie Studien, die den Entzug von Gelegenheitsrauchern und Rauchern miteinander vergleichen. 
Insbesondere mangelt es an Langzeitstudien, die den Verlauf polysomnografischer oder neuroendokriner Parameter beobachten. Hierfür sollten Studien durchgeführt werden, die sich in der Methodik soweit ähneln, dass ein sinnvoller Vergleich und die Erstellung von Metaanalysen möglich sind. Auch sollten die Werte der rückfälligen Probanden mit in die Betrachtung einbezogen werden, um mögliche Prädiktoren ermitteln zu können. Hierbei ist zu beachten, dass natürlich eine hohe Evidenzklasse des Studiendesigns wünschenswert ist, jedoch oft auf Fall-Kontroll-Studien zurückgegriffen werden muss, da ein Nikotinentzug nicht doppelblind durchgeführt werden kann. 


\section{Zusammenfassung}

Der Konsum von Tabakprodukten ist trotz der bekannten gesundheitlichen Folgen weltweit verbreitet. Nikotin ist der entscheidende Inhaltsstoff des Tabaks, der die Entwicklung und Aufrechterhaltung einer Abhängigkeit fördert. Die Nikotinabhängigkeit ist u. a. gekennzeichnet durch den wiederholten Konsum von Tabak und das Auftreten von Entzugserscheinungen in Abstinenz. Schlafstörungen, Reizbarkeit und schlechte Stimmung gehören zu den häufigsten Symptomen, die im Nikotinentzug auftreten können. Die neurobiologischen Korrelate hierfür spiegeln sich möglicherweise in polysomnografischen und endokrinen Parametern wider.

Da Nikotin wie auch der Nikotinentzug die verschiedensten zentralnervösen Systeme beeinflusst, die auch Teil der Schlafregulation, des dopaminergen Systems oder der HHN-Achse sind, ist eine Beobachtung von polysomnografischen und endokrinen Messgrößen zweckmäßig, um die Auswirkungen eines Nikotinkonsums und eines Nikotinentzugs auf den Organismus zu analysieren.

Diese Übersichtsarbeit betrachtet den Schlaf, das dopaminerge System und die Transmitter der HHN-Achse gemeinsam, da es eine enge Verbindung zwischen dem Schlaf und den genannten Systemen gibt. So wird der Schlaf auch durch jene Transmitter reguliert, auf die Nikotin einen Einfluss hat.

Das Verständnis der Veränderungen, die durch Nikotin bewirkt werden, sowie die Kenntnis über den Verlauf eines Entzugs können dazu beitragen, die Nikotinabhängigkeit besser zu verstehen und möglicherweise Prädiktoren für einen erfolgreichen Entzug oder einen Rückfall zu identifizieren. Mit den geeigneten Maßnahmen könnte die von vielen Rauchern angestrebte Abstinenz erleichtert werden.

Die vorliegende Arbeit bietet durch eine systematische Literaturrecherche eine Übersicht über die bisher durchgeführten Studien, die sich mit den Konsequenzen einer Nikotingabe und eines 
Nikotinentzugs auf den Schlaf, das dopaminerge System und die HHN-Achse beschäftigt haben. Die Recherche wurde in der elektronischen Datenbank PubMed vorgenommen. Eingeschlossen wurden alle Originalstudien, die den definierten Kriterien entsprechen und bis einschließlich 15. August 2012 publiziert wurden. Konkret wurde nach Studien gesucht, die die genannten Parameter nach einer Nikotingabe an Tiere oder Nichtraucher oder im Nikotinentzug von Gelegenheitsrauchern oder Rauchern untersucht haben. Insgesamt konnten 3037 Veröffentlichungen gefunden werden, von denen 93 den Suchkriterien entsprechen.

Aus den tierexperimentellen Arbeiten ging hervor, dass Nikotin eine dosisabhängige Wirkung auf den Schlaf hatte, wobei geringere Dosen den REM-Schlaf und höhere Dosen die Wachheit förderten. Durch Nikotin kam es zudem zu einer vermehrten Ausschüttung von Hormonen der HHN-Achse. Nichtraucher zeigten nach einer Nikotingabe eine Verminderung des REMSchlafs. Bei depressiven Nichtrauchern konnte hingegen eine Verbesserung der Schlafqualität und der Depressionen beobachtet werden. Studien, die sich mit dem dopaminergen System von Nichtrauchern auseinandergesetzt haben, wurden nicht gefunden. Die Freisetzung von Hormonen der HHN-Achse wurde auch bei Nichtrauchern durch Nikotin stimuliert. Die Suche nach Studien, die sich mit Gelegenheitsrauchern beschäftigten, blieb ergebnislos. Der Entzug bei Rauchern zeigte in den ersten Entzugstagen eine erhöhte Fragmentierung des Schlafs, die durch den Einsatz von Nikotinersatztherapien gemindert werden konnte. In der einzigen Langzeitstudie, die sich mit dem Entzug von Rauchern beschäftigte, wurde eine Zunahme des REM-Schlafs und eine Abnahme der Gesamtschlafzeit beobachtet. Die Parameter des dopaminergen Systems waren im Entzug von wenigen Stunden stabil, eine Langzeitstudie zeigte jedoch in der PET eine Zunahme des Bindungspotentials an Dopaminrezeptoren. Die Konzentration der Hormone der HHN-Achse fiel im längerfristigen Nikotinentzug ab.

Zusammengenommen kann aus der gefundenen Literatur aufgrund der geringen Studienzahl und den sehr unterschiedlichen Studienmethoden keine Metaanalyse erstellt werden. Zudem konnten aus den Ergebnissen nur ein möglicher Prädiktor für den Verlauf eines Nikotinentzugs identifiziert, ein weiterer ausgeschlossen werden. Der Verlauf der Cortisolkonzentration im Entzug war in einigen Studien mit der Stärke von Entzugserscheinungen korreliert und könnte als Prädiktor geeignet sein: Je höher der Konzentrationsabfall, desto stärker die Symptome, die einen Rückfall 
fördern. Das Bindungspotential an D1-Rezeptoren im Striatum vor dem Entzug stellt keinen prädestinierenden Faktor einer erfolgreichen Abstinenz oder eines Rückfalls dar.

Durch die Übersichtsarbeit konnten Lücken in der Nikotinforschung aufgedeckt werden. Insbesondere fehlen Studien, die sich mit dem dopaminergen System, mit Nichtrauchern nach einer Nikotingabe und Gelegenheitsrauchern im Entzug auseinandersetzen, sowie Studien, die einen längerfristigen Nikotinentzug bezüglich des Schlafs untersuchen. 
6 Anhang: Tabellen 6.1-6.7 


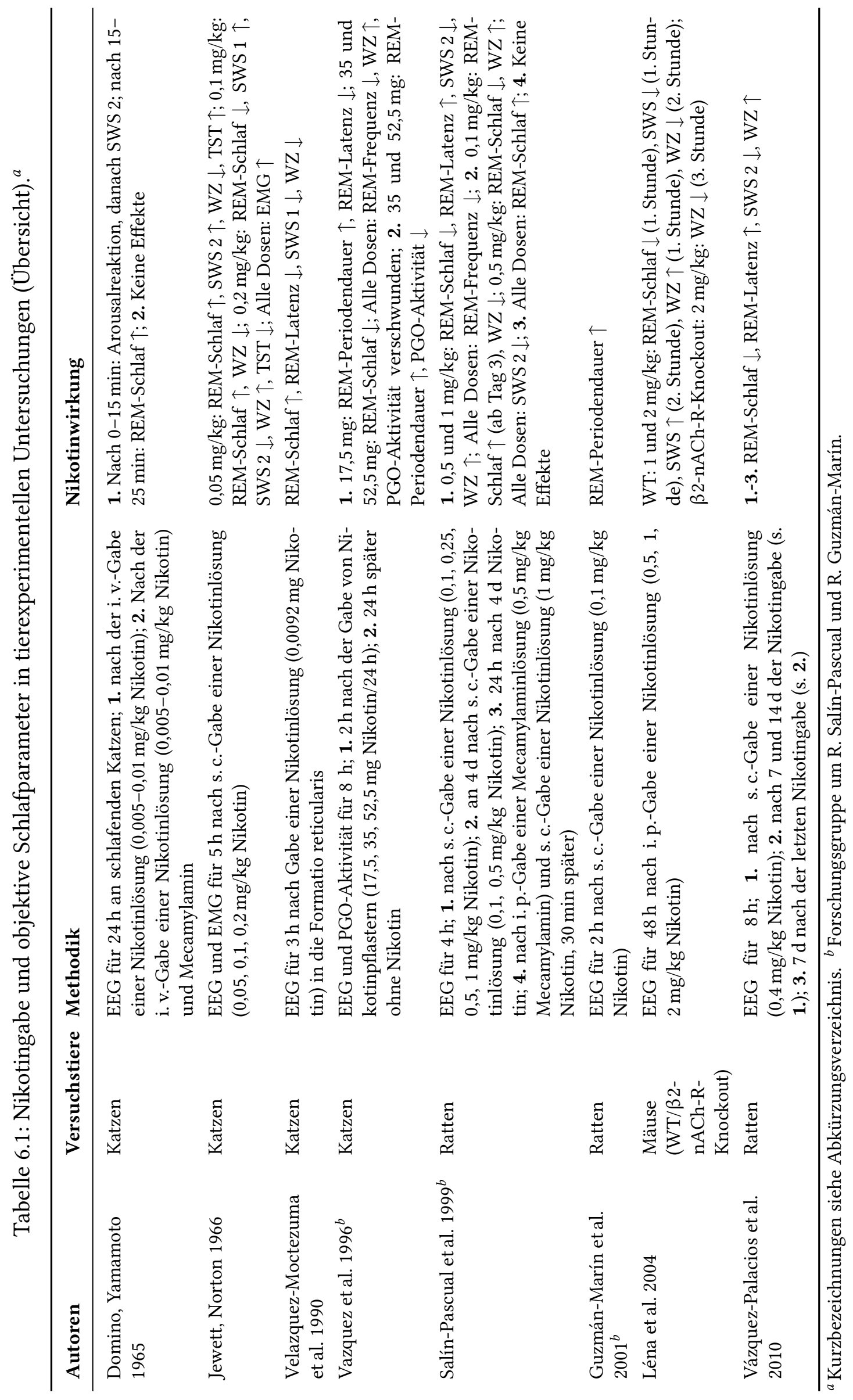




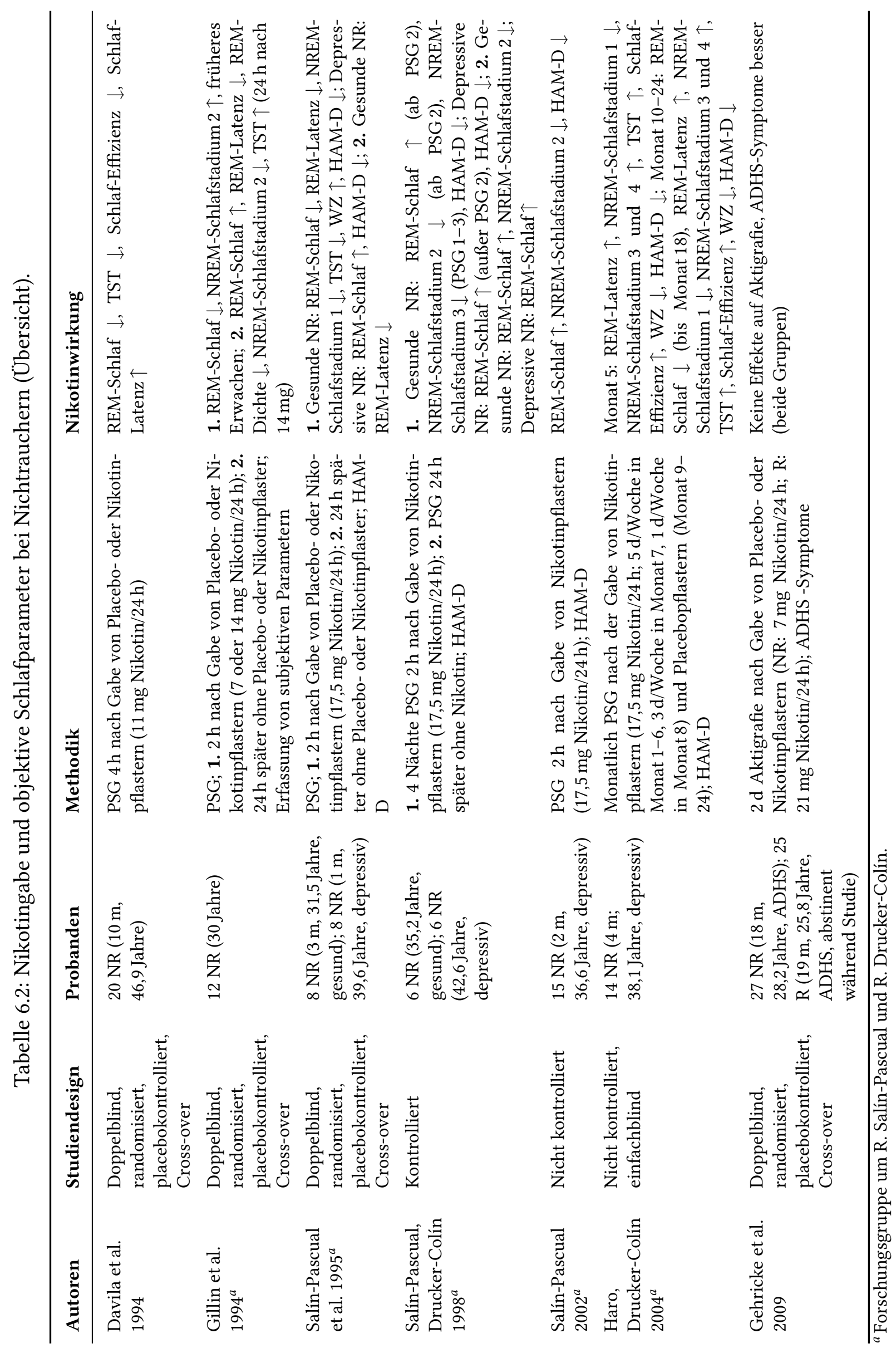




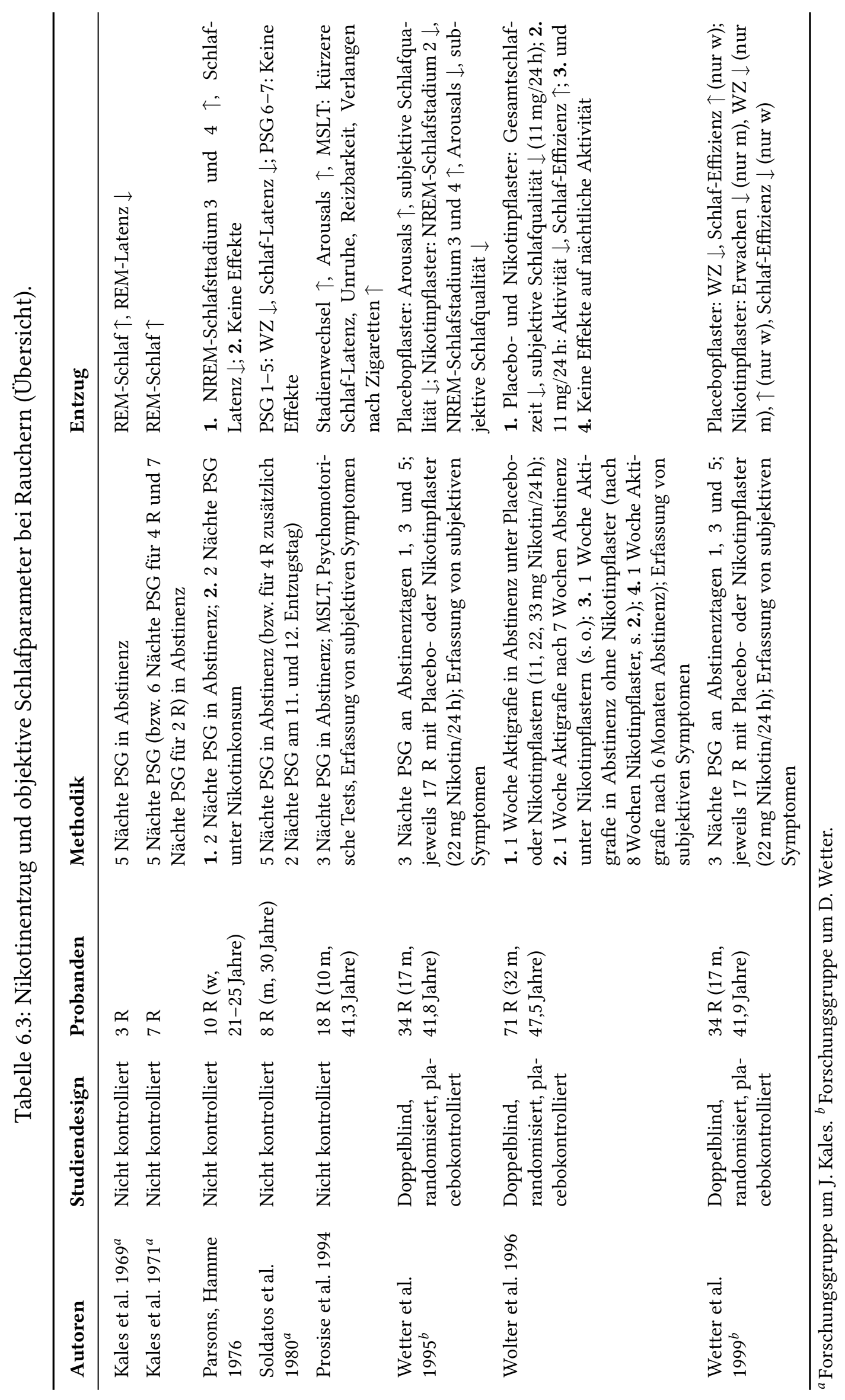




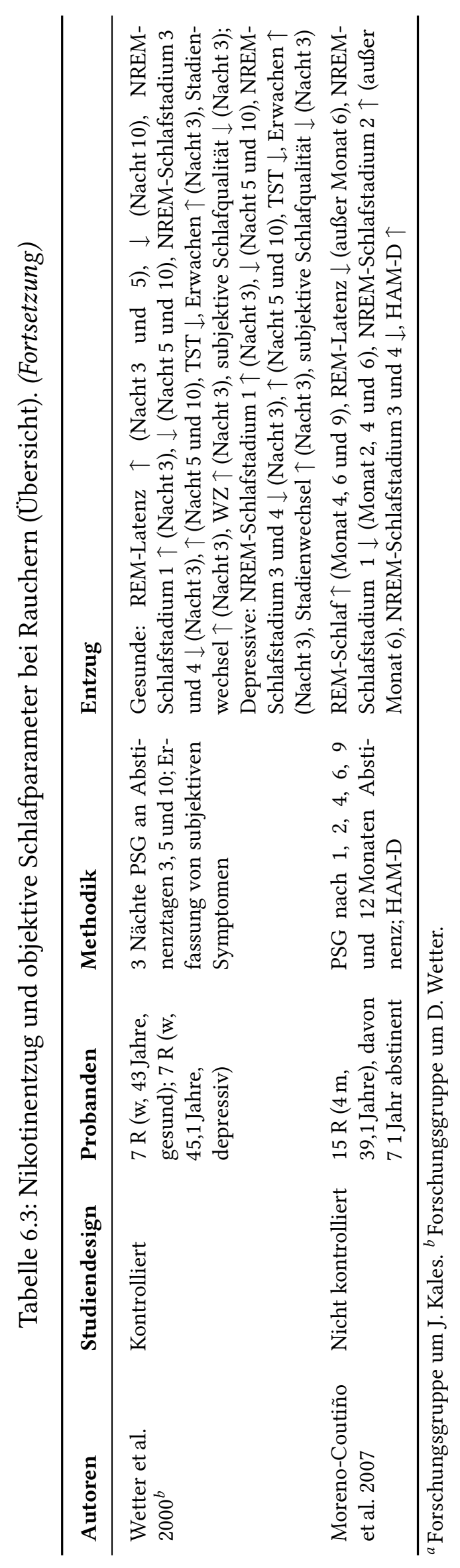




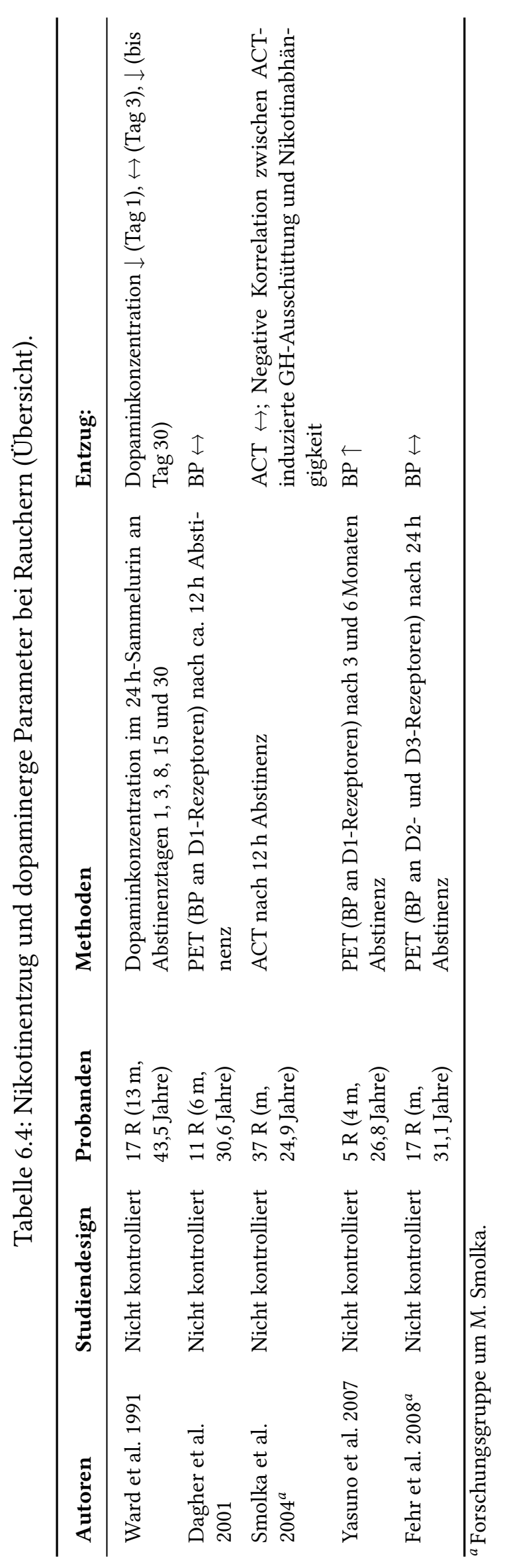




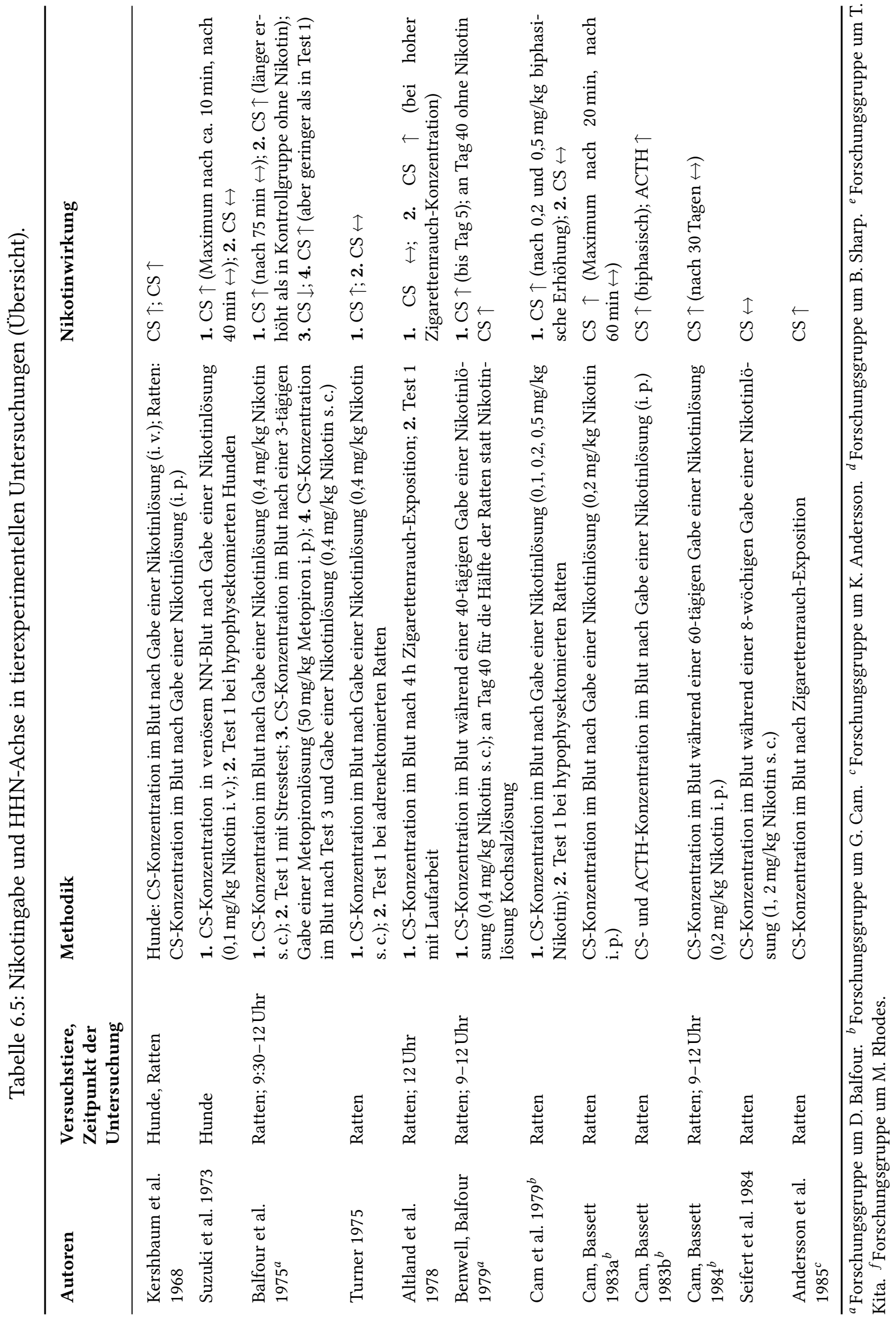




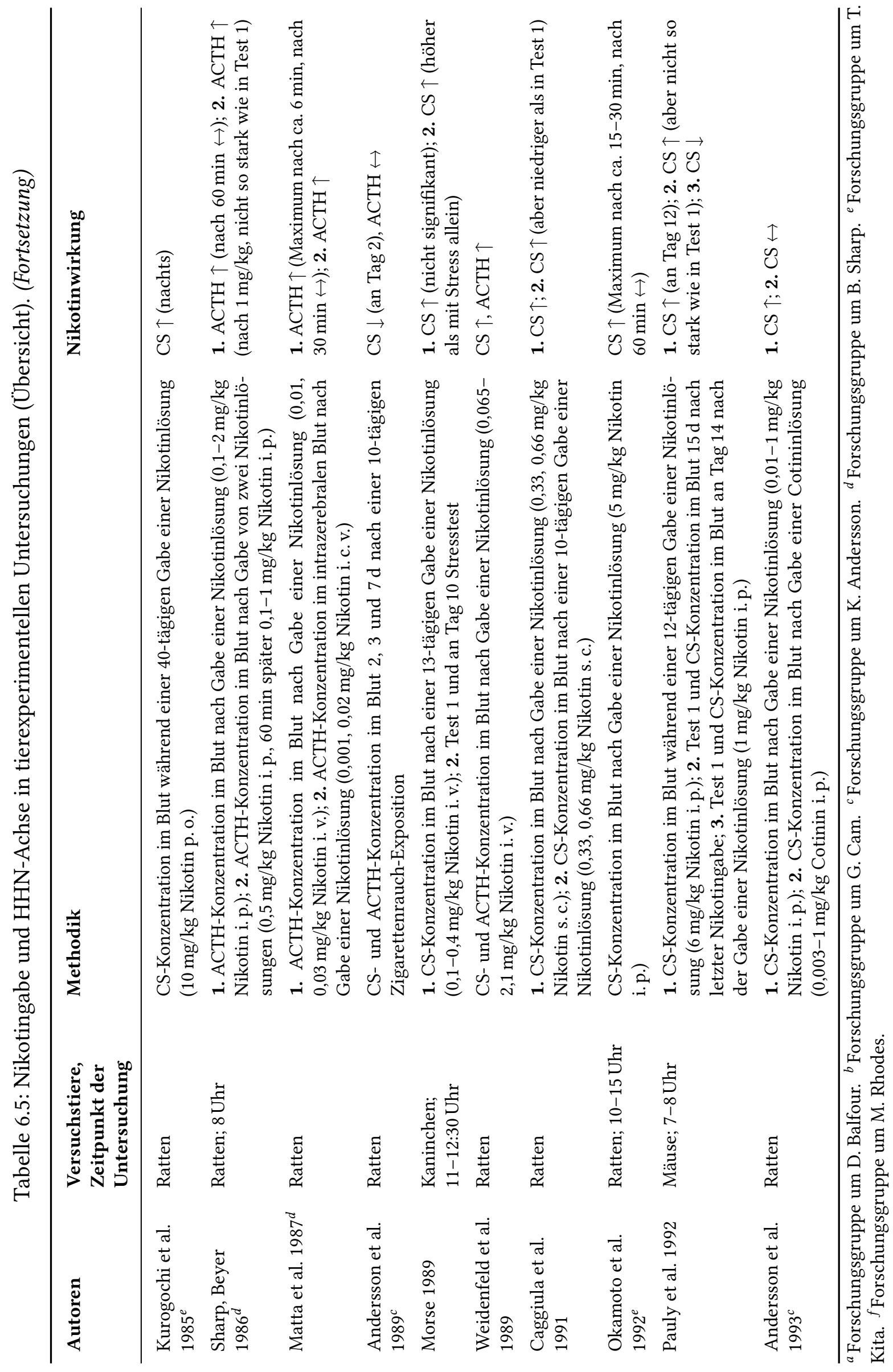




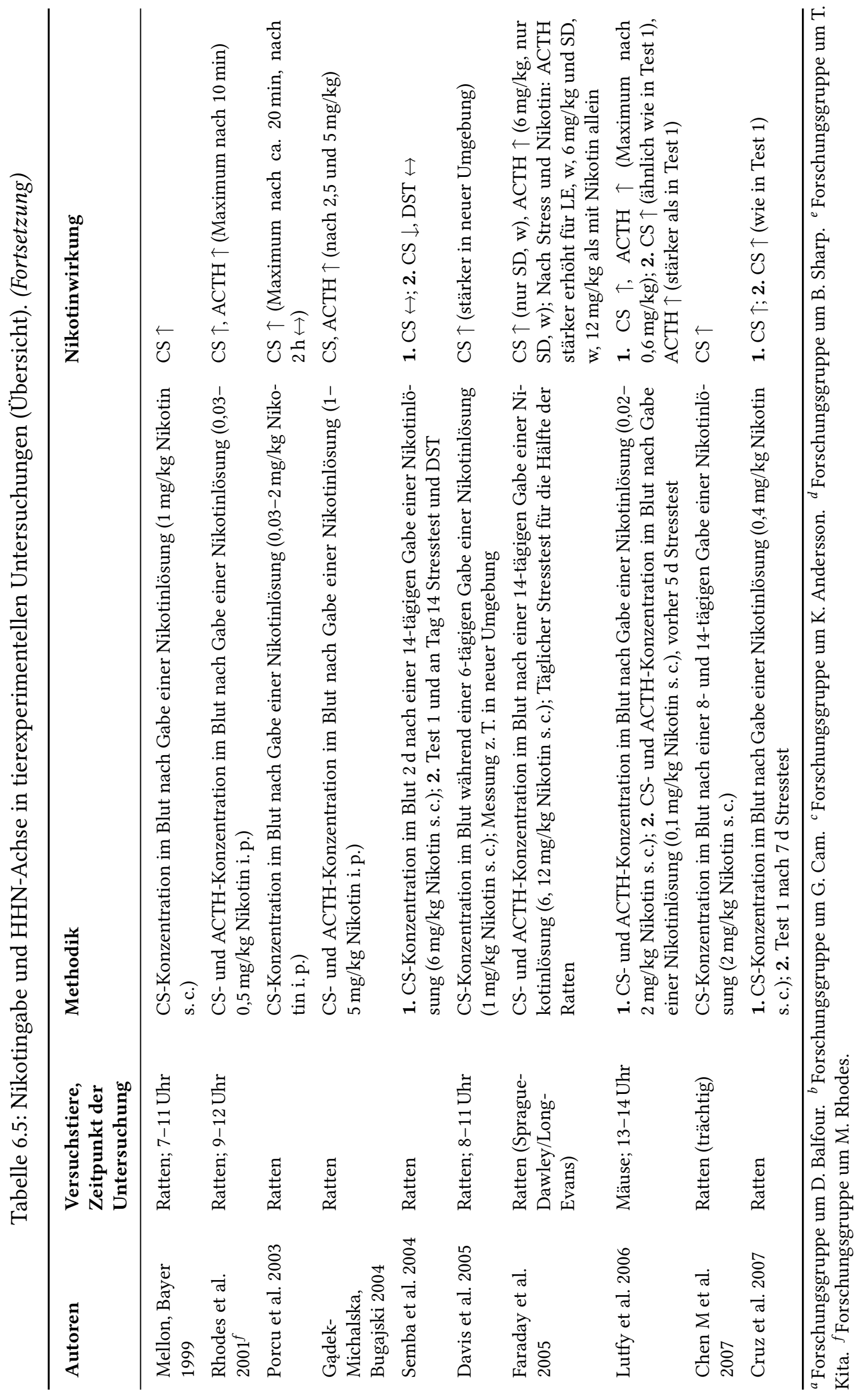




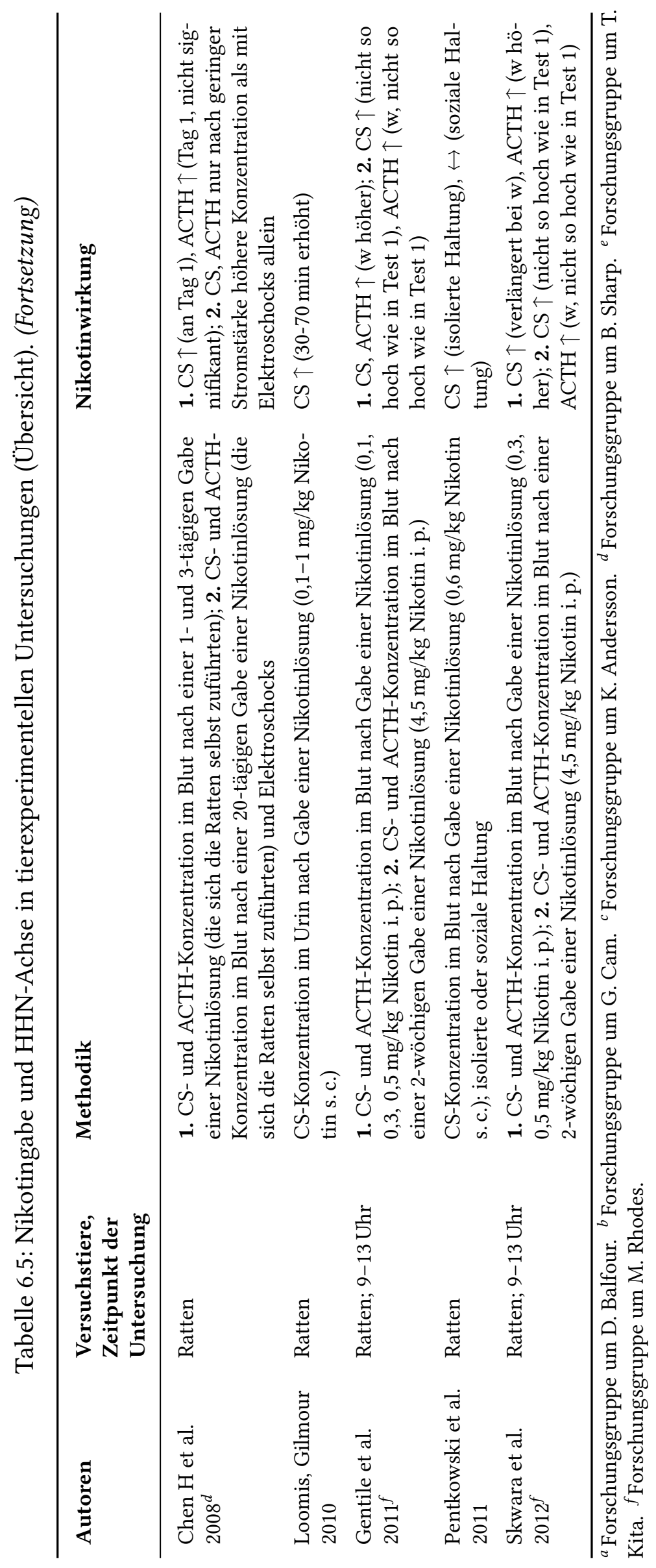




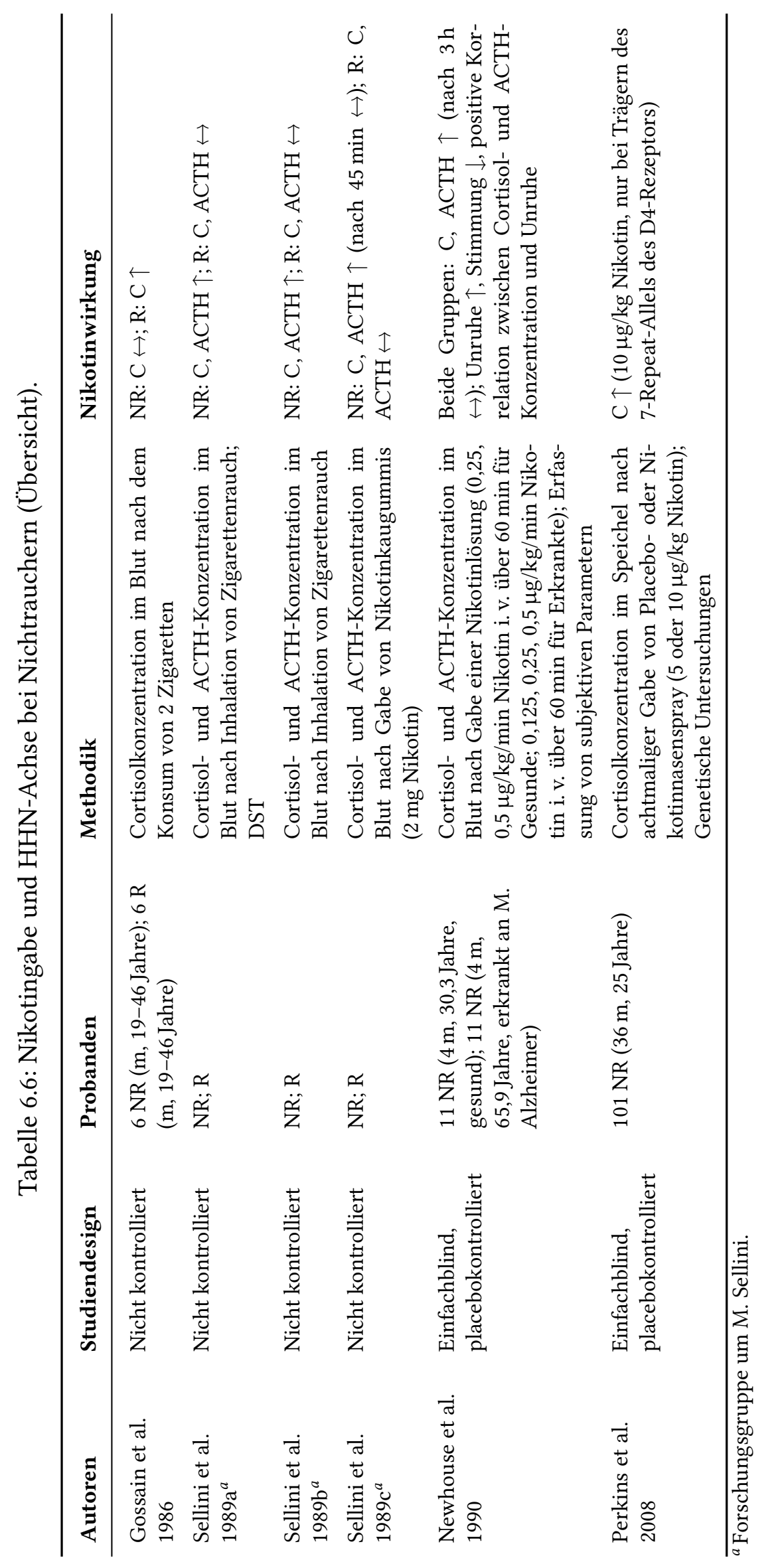




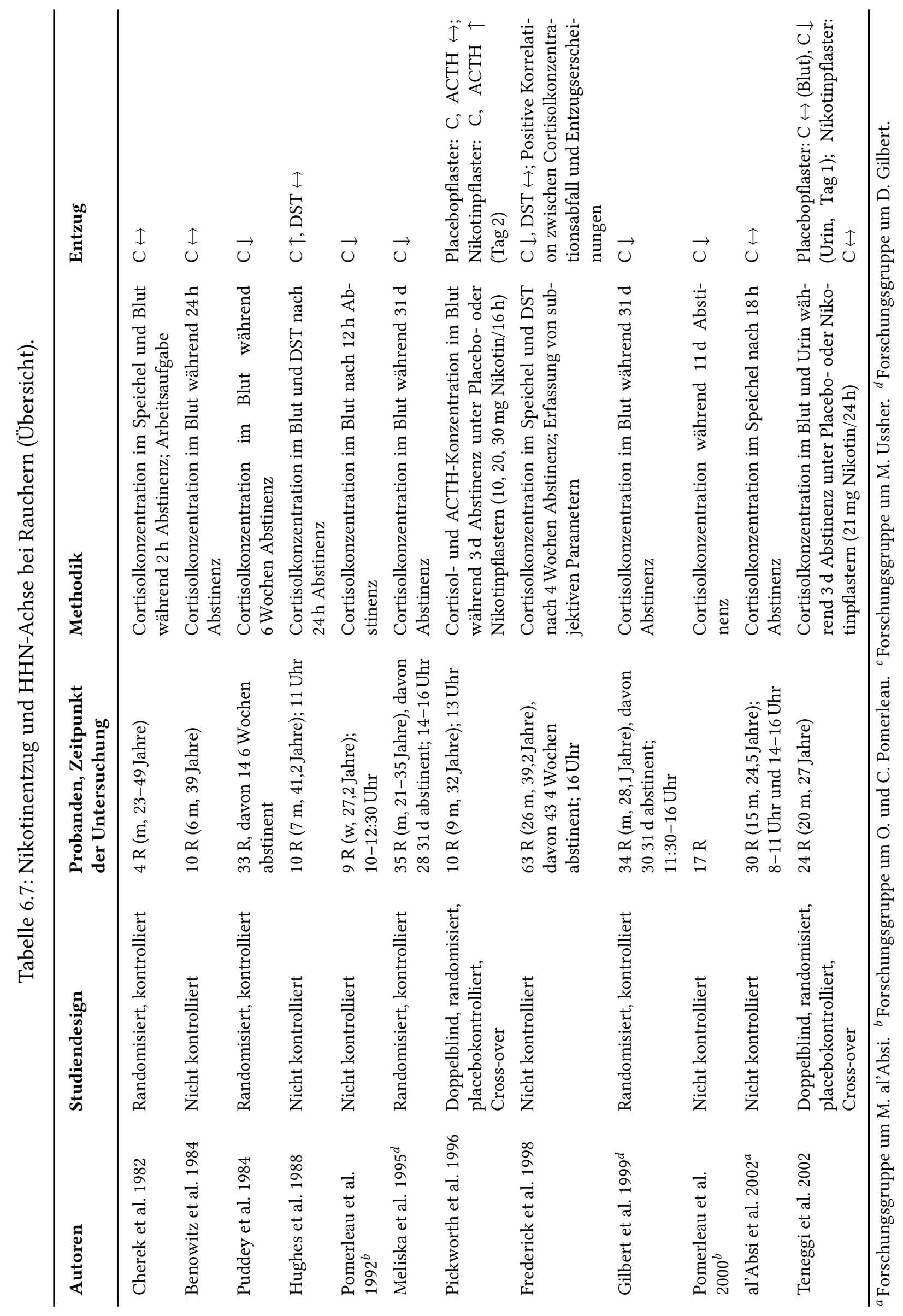




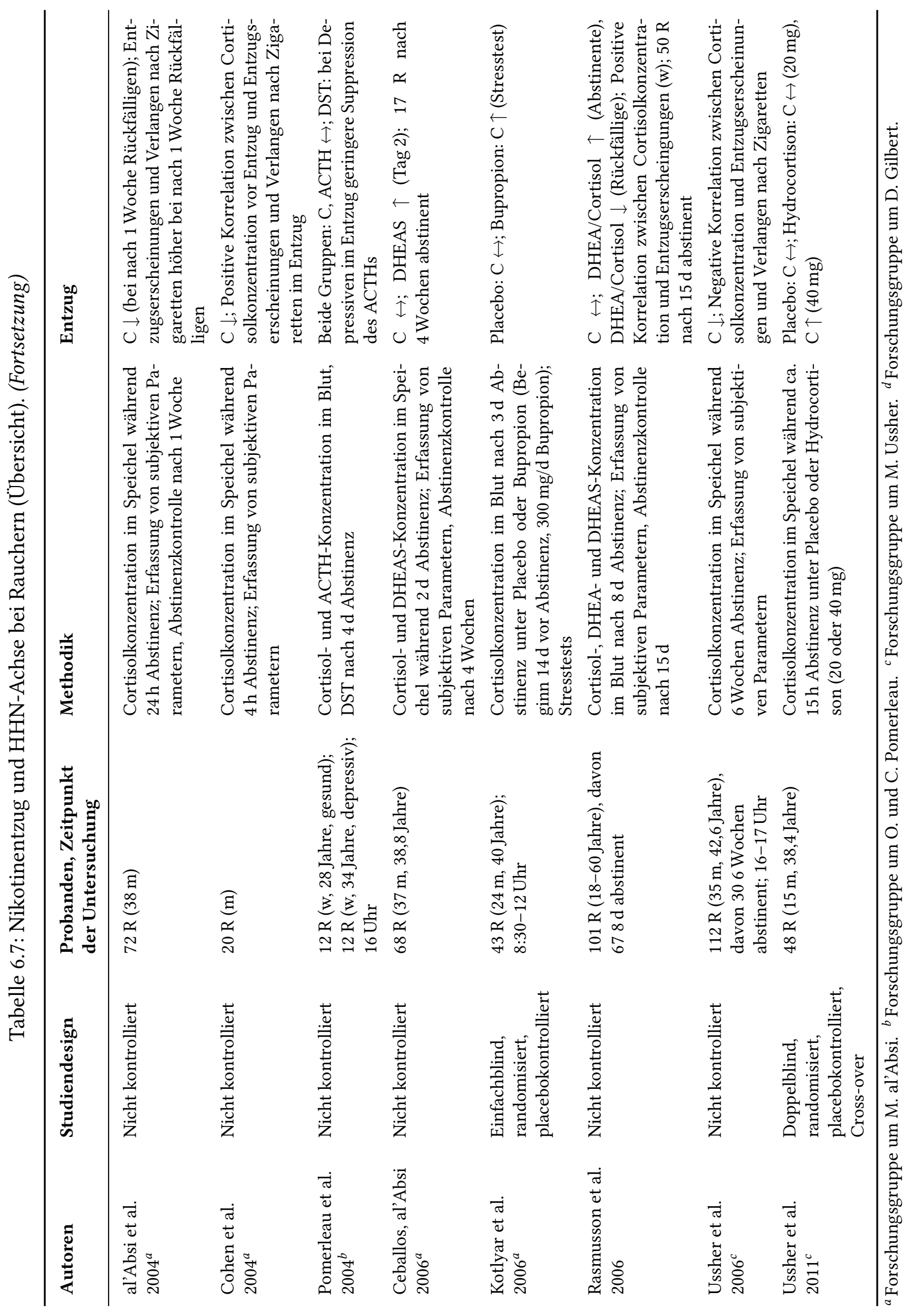




\section{Literaturverzeichnis}

al'Absi M, Amunrud T und Wittmers LE (2002): Psychophysiological effects of nicotine abstinence and behavioral challenges in habitual smokers. Pharmacol Biochem Behav $\underline{72}, 707-716$

al'Absi M, Wittmers LE, Erickson J, Hatsukami D und Crouse B (2003): Attenuated adrenocortical and blood pressure responses to psychological stress in ad libitum and abstinent smokers. Pharmacol Biochem Behav 74, 401-410

al'Absi M, Hatsukami D, Davis GL und Wittmers LE (2004): Prospective examination of effects of smoking abstinence on cortisol and withdrawal symptoms as predictors of early smoking relapse. Drug Alcohol Depend $\underline{73}$, 267-678

al'Absi M, Hatsukami D und Davis GL (2005): Attenuated adrenocorticotropic responses to psychological stress are associated with early smoking relapse. Psychopharmacology (Berl) $\underline{181}$, $107-117$

Alóe F, de Azevedo AP und Hasan R (2005): Sleep-wake cycle mechanisms. Rev Bras Psiquiatr 27, $33-39$

Altland PD, Parker MG und Brubach HF (1978): Effects of Graded Concentrations of Cigarette Smoke on Plasma Enzyme, Corticosterone, and Body Temperature in Rats. Environ Res $\underline{15}$, $160-169$

Andersson K, Fuxe K, Eneroth P, Mascagni F und Agnati LF (1985): Effects of acute intermittent exposure to cigarette smoke on catecholamine levels and turnover in various types of hypothalamic DA and NA nerve terminal systems as well as on the secretion of adenohypophyseal hormones and corticosterone. Acta Physiol Scand 124, 277-285 
Andersson K, Fuxe K, Eneroth P, Jansson A und Härfstrand A (1989): Effects of withdrawal from chronic exposure to cigarette smoke on hypothalamic and preoptic catecholamine nerve terminal systems and on the secretion of pituitary hormones in the male rat. Naunyn Schmiedebergs Arch Pharmacol 339, 387-396

Andersson K, Jansson A, Kuylenstierna F und Eneroth P (1993): Nicotine and its major metabolite cotinine have different effects on aldosterone and prolactin serum levels in the normal male rat. Eur J Pharmacol 228, 305-312

Aubin HJ, Luthringer R, Demazières A, Dupont C und Lagrue G (2006): Comparison of the effects of a 24-hour nicotine patch and a 16-hour nicotine patch on smoking urges and sleep. Nicotine Tob Res $\underline{8}, 193-201$

Badrick E, Kirschbaum C und Kumari M (2007): The Relationship between Smoking Status and Cortisol Secretion. J Clin Endocrinol Metab 92, 819-824

Balfour DJK, Khullar AK und Longden A (1975): Effects of Nicotine on Plasma Corticosterone and Brain Amines in Stressed and Unstressed Rats. Pharmacol Biochem Behav $\underline{3}, 179-184$

Baron JA, Comi RJ, Cryns V, Brinck-Johnsen T und Mercer NG (1995): The Effect of Cigarette Smoking on Adrenal Cortical Hormones. J Pharmacol Exp Ther 272, 151-155

Batra A: Tabakabhängigkeit. Steinkopff Verlag, Darmstadt 2000

Becker-Carus C: Psychische Aktivität im REM-Schlaf. In: Schulz H, Geisler P und Rodenbeck A, Kompendium Schlafmedizin. ecomed Medizin, Landsberg 2011, Seite 1-2

Benowitz NL (2008a): Clinical Pharmacology of Nicotine: Implications for Understanding, Preventing, and Treating Tobacco Addiction. Clin Pharmacol Ther $\underline{83}$, 531-541

Benowitz NL (2008b): Neurobiology of Nicotine Addiction: Implications for Smoking Cessation Treatment. Am J Med 121, S3-S10

Benowitz NL und Jacob P (1984): Daily intake of nicotine during cigarette smoking. Clin Pharmacol Ther $\underline{35}, 499-504$ 
Benowitz NL, Jacob P, Jones RT und Rosenberg J (1982): Interindividual variability in the metabolism and cardiovascular effects of nicotine in man. J Pharmacol Exp Ther 221, 368-372

Benowitz NL, Kuyt F und Jacob P (1984): Influence of nicotine on cardiovascular and hormonal effects of cigarette smoking. Clin Pharmacol Ther $\underline{36}$, 74-81

Benwell ME und Balfour DJK (1979): Effects of Nicotine Administration and Its Withdrawal on Plasma Corticosterone and Brain 5-Hydroxyindoles. Psychopharmacology (Berl) $\underline{63}$, 7-11

Berlin I (2009): Endocrine and metabolic effects of smoking cessation. Curr Med Res Opin $\underline{25}$, $527-534$

Böcker W, Denk H und Heitz PU: Pathologie, 3. Aufl. Urban und Fischer Verlag, München 2004

Borbély A (1982): A two process model of sleep regulation. Hum Neurobiol 1, 195-204

Borbély A: Schlaf als regulierter Prozess. In: Schulz H, Geisler P und Rodenbeck A, Kompendium Schlafmedizin. ecomed Medizin, Landsberg 2004, Seite 1-4

Born J, Rasch B und Gais S (2006): Sleep to Remember. Neuroscientist 12, 410-424

Breese GR, Sinha R und Heilig M (2011): Chronic alcohol neuroadaptation and stress contribute to susceptibility for alcohol craving and relapse. Pharmacol Ther $\underline{129}, 149-171$

Brody AL (2006): Functional brain imaging of tobacco use and dependence. J Psychiatr Res $\underline{40}$, $404-418$

Caggiula AR, Epstein LH, Antelman SM, Saylor SS, Perkins KA, Knopf S and Stiller R (1991): Conditioned Tolerance to the Anorectic and Corticosterone-Elevating Effects of Nicotine. Pharmacol Biochem Behav $\underline{40}, 53-59$

Cam GR und Bassett JR (1983a): The Effect of Acute Nicotine Administration on Plasma Levels of the Thyroid Hormones and Corticosterone in the Rat. Pharmacol Biochem Behav 19, 559-561

Cam GR und Bassett JR (1983b): The plasma levels of ACTH following exposure to stress or nicotine. Arch Int Pharmacodyn Ther 264, 154-167 
Cam GR und Bassett JR (1984): Effect of Prolonged Exposure to Nicotine and Stress on the PituitaryAdrenocortical Response; the Possibility of Cross-Adaptation. Pharmacol Biochem Behav $\underline{20}$, $221-226$

Cam GR, Bassett JR und Cairncross KD (1979): The action of nicotine on the pituitary-adrenal cortical axis. Arch Int Pharmacodyn Ther 237, 49-66

CDC/National Center for Health Statistics: Adult Tobacco Use Information, Website vom 17.06.2009 (http://www.cdc.gov/nchs/nhis/tobacco/tobacco_glossary.htm)

Ceballos NA und al'Absi M (2006): Dehydroepiandrosterone sulfate, cortisol, mood state and smoking cessation: Relationship to relapse status at 4-week follow-up. Pharmacol Biochem Behav $\underline{85}, 23-28$

Chen H, Fu Y und Sharp BM (2008): Chronic Nicotine Self-Administration Augments HypothalamicPituitary-Adrenal Responses to Mild Acute Stress. Neuropsychopharmacology $\underline{33}, 721-730$

Chen M, Wang T, Liao ZX, Pan XL, Feng YH und Wang H (2007): Nicotine-induced prenatal overexposure to maternal glucocorticoid and intrauterine growth retardation in rat. Exp Toxicol Pathol $\underline{59}, 245-251$

Cherek DR, Smith JE, Lane JD und Brauchi JT (1982): Effects of cigarettes on saliva cortisol levels. Clin Pharmacol Ther $\underline{32}, 765-768$

Chi YW und Jaff MR (2008): Optimal Risk Factor Modification and Medical Management of the Patient With Peripheral Arterial Disease. Catheter Cardiovasc Interv $\underline{71}$, 475-489

Cinciripini PM, Wetter DW, Tomlinson GE, Tsoh JY, De Moor CA, Cinciripini LG und Minna JD (2004): The effects of the DRD2 polymorphism on smoking cessation and negative affect: Evidence for a pharmacogenetic effect on mood. Nicotine Tob Res $\underline{6}, 229-239$

Cohen LM, al'Absi M und Collins, Jr FL (2004): Salivary cortisol concentrations are associated with acute nicotine withdrawal. Addict Behav 29, 1673-1678

Cohrs S, Rodenbeck A, Riemann D, Szagun B, Jaehne A, Brinkmeyer J, Gründer G, Wienker T, DiazLacava A und Mobascher A (2012): Impaired sleep quality and sleep duration in smokers-results from the German Multicenter Study on Nicotine Dependence. Addict Biol, im Druck 
Cornuz J, Humair JP und Zellweger JP (2004): Tabakentwöhnung. Schweiz Med Forum $\underline{4}$, 764-770

Cruz FC, DeLucia R und Planeta CS (2008): Effects of chronic stress on nicotine-induced locomotor activity and corticosterone release in adult and adolescent rats. Addict Biol $\underline{13}, 63-69$

Daan S, Beersma DG und Borbély AA (1984): Timing of human sleep : recovery process gated by a circadian pacemaker. Am J Physiol 246, R161-R183

Dagher A, Bleicher C, Aston JAD, Gunn RN, Clarke PBS und Cumming P (2001): Reduced Dopamine D1 Receptor Binding in the Ventral Striatum of Cigarette Smokers. Synapse $\underline{42}, 48-53$

Damsma G, Day J und Fibiger HC (1989): Lack of tolerance to nicotine-induced dopamine release in the nucleus accumbens. Eur J Pharmacol 168, 363-368

Dani JA und Heinemann S (1996): Molecular and Cellular Aspects of Nicotine Abuse. Neuron $\underline{16}$, 905-908

Davila DG, Hurt RD, Offord KP, Harris CD und Shepard JW (1994): Acute Effects of Transdermal Nicotine on Sleep Architecture, Snoring, and Sleep-disordered Breathing in Nonsmokers. Am J Respir Crit Care Med 150, 469-474

Davis KW, Cepeda-Benito A, Harraid JH und Wellman PJ (2005): Plasma corticosterone in the rat in response to nicotine and saline injections in a context previously paired or unpaired with nicotine. Psychopharmacology (Berl) $\underline{180}, 466-472$

De Biasi M und Dani JA (2011): Reward, Addicition, Withdrawal to Nicotine. Annu Rev Neurosci 34, 105-130

Dekant W und Vamvakas S: Toxikologie, 2. Aufl. Spektrum Akademischer Verlag, München 2005

Doll R, Peto R, Boreham J und Sutherland I (2004): Mortality in relation to smoking: 50 years' observations on male British doctors. BMJ $\underline{328}, 1519-1528$

Domino EF und Yamamoto K (1965): Nicotine: Effect on the Sleep Cycle of the Cat. Science 150, 637-638 
Dušková M, Šimůnková K, Hill M, Hruškovičová H, Hoskovcová P, Králíková E und Stárka L (2010): Higher levels of salivary $\alpha$-amylase predict failure of cessation efforts in male smokers. Physiol Res $\underline{59}, 765-771$

Faraday MM, Blakeman KH und Grunberg NE (2005): Strain and sex alter effects of stress and nicotine on feeding, body weight, and HPA axis hormones. Pharmacol Biochem Behav $\underline{80}$, $577-589$

Fehr C, Yakushev I, Hohmann N, Buchholz HG, Landvogt C, Deckers H, Eberhardt A, Kläger M, Smolka MN, Scheurich A et al. (2008): Association of Low Striatal Dopamine D2 Receptor Availability With Nicotine Dependence Similar to That Seen With Other Drugs of Abuse. Am J Psychiatry $\underline{165}, 507-514$

Frederick SL, Reus VI, Ginsberg D, Hall SM, Munoz RF und Ellman G (1998): Cortisol and Response to Dexamethasone as Predictors of Withdrawal Distress and Abstinence Success in Smokers. Biol Psychiatry $\underline{43}, 525-530$

Fredrickson PA, Hurt RD, Lee GM, Wingender L, Croghan IT, Lauger G, Gomez-Dahl L und Offord KP (1995): High dose transdermal nicotine therapy for heavy smokers: safety, tolerability and measurement of nicotine and cotinine levels. Psychopharmacology (Berl) 122, 215-222

Gądek-Michalska A und Bugajski J (2004): Role of nitric oxide in the nicotine-induced pituitaryadrenocortical response. J Physiol Pharmacol $\underline{55}$, 443-455

Gann H, Feige B, Fasihi S, van Calker D, Voderholzer U und Riemann D (2002): Periodic limb movements during sleep in alcohol dependent patients. Eur Arch Psychiatry Clin Neurosci 252, $124-129$

Gehricke JG, Hong N, Whalen CK, Steinhoff K und Wigal TL (2009): Effects of Transdermal Nicotine on Symptoms, Moods, and Cardiovascular Activity in the Everyday Lives of Smokers and Nonsmokers With Attention-Deficit/Hyperactivity Disorder. Psychol Addict Behav 23, $644-655$ 
Gentile NE, Andrekanic JD, Karwoski TE, Czambel RK, Rubin RT und Rhodes ME (2011): Sexually diergic hypothalamic-pituitary-adrenal (HPA) responses to single-dose nicotine, continuous nicotine infusion, and nicotine withdrawal by mecamylamine in rats. Brain Res Bull $\underline{85}, 145-152$

Gilbert DG, Meliska CJ, Williams CL und Jensen RA (1992): Subjective correlates of cigarettesmoking-induced elevations of peripheral beta-endorphin and cortisol. Psychopharmacology (Berl) $106,275-281$

Gilbert DG, McClernon FJ, Rabinovich NE, Dibb WD, Plath LC, Hiyane S, Jensen RA, Meliska CJ, Estes SL und Gehlbach BA (1999): EEG, Physiology, and Task-Related Mood Fail to Resolve Across 31 Days of Smoking Abstinence: Relations to Depressive Traits, Nicotine Exposure, and Dependence. Exp Clin Psychopharmacol ㅁ, 427-443

Gillin JC, Lardon M, Ruiz C, Golshan S und Salín-Pascual R (1994): Dose-Dependent Effects of Transdermal Nicotine on Early Morning Awakening and Rapid Eye Movement Sleep Time in Nonsmoking Normal Volunteers. J Clin Psychopharmacol 14, 264-267

Glassman AH, Helzer JE, Covey LS, Cottler LB, Stetner F, Tipp JE und Johnson J (1990): Smoking, smoking cessation, and major depression. JAMA $\underline{264}, 1546-1549$

Gossain VV, Sherma NK, Srivastava L, Michelakis AM und Rovner DR (1986): Hormonal Effects of Smoking-II: Effects on Plasma Cortisol, Growth Hormone, and Prolactin. Am J Med Sci 291, $325-327$

Guzmán-Marín R, Alam MN, Mihailescu S, Szymusiak R, McGinty D and Drucker-Colín R (2001): Subcutaneous administration of nicotine changes dorsal raphe serotonergic neurons discharge rate during REM sleep. Brain Res $\underline{888}, 321-325$

Hajak G und Rüther E: Insomnie. Springer Verlag, Berlin 1995

Happe S und Walther BW: Schlafmedizin in der Praxis. Ecomed Medizin, Heidelberg 2009

Haro R und Drucker-Colín R (2004): A Two-Year Study on the Effects of Nicotine and its Withdrawal on Mood and Sleep. Pharmacopsychiatry $\underline{37}, 221-227$

Haustein KO: Tabakabhängigkeit. Deutscher Ärzte-Verlag, Köln 2001 
Heath AC, Bucholz KK, Madden PAF, Dinwiddie SH, Slutske WS, Bierut LJ, Statham DJ, Dunne MP, Whitfield JB und Martin NG (1997): Genetic and environmental contributions to alcohol dependence risk in a national twin sample: consistency of findings in women and men. Psychol Med 27, 1381-1396

Heatherton TF, Kozlowski LT, Frecker RC und Fagerström KO (1991): The Fagerström Test for Nicotine Dependence: a revision of the Fagerström Tolerance Questionnaire. Br J Addict $\underline{86}$, $1119-1127$

Heinz A und Batra A: Neurobiologie der Alkohol- und Nikotinabhängigkeit. W. Kohlhammer, Stuttgart 2003

Heinz A, Siessmeier T, Wrase J, Hermann D, Klein S, Grüsser SM, Grüsser-Sinopoli SM, Flor H, Braus DF, Buchholz HG et al. (2004): Correlation Between Dopamine D2 Receptors in the Ventral Striatum and Central Processing of Alcohol Cues and Craving. Am J Psychiatry 161, 1783-1789

Hu B, Bouhassira D, Steriade M und Deschênes M (1988): The blockage of ponto-geniculo-occipital waves in the cat lateral geniculate nucleus by nicotinic antagonists. Brain Res $\underline{473}, 394-397$

Hughes JR (1992): Tobacco Withdrawal in Self-Quitters. J Consult Clin Psychol 60, 689-697

Hughes JR, Arana G, Amori G, Stewart F und Workman R (1988): Effect of Tobacco Withdrawal on the Dexamethasone Suppresion Test. Biol Psychiatry 23, 96-98

Hukkanen J, Jacob P und Benowitz NL (2005): Metabolism and Disposition Kinetics of Nicotine. Pharmacol Rev $\underline{57}, 79-115$

Imperato A, Mulas A und Di Chiara G (1986): Nicotine preferentially stimulates dopamine release in the limbic system of freely moving rats. Eur J Pharmacol $\underline{132}, 337-338$

Imperial Cancer Research Fund General Practice Research Group (1993): Effectiveness of a nicotine patch in helping people stop smoking: results of a randomised trial in general practice. BMJ $\underline{306}$, 1304-1308

Jähne A, Cohrs S, Rodenbeck A, Andreas S, Loessl B, Feige B, Kloepfer C, Hornyak M und Riemann D (2010): Nikotin. Einfluss auf den Schlaf und Relevanz für Psychiatrie und Psychotherapie. Nervenarzt $\underline{81}, 844-859$ 
Jain A (2003): Treating nicotine addiction. BMJ 327, 1394-1396

Janowsky DS, El-Yousef MK, Davis JM und Sekerke HJ (1972): A cholinergic-adrenergic hypothesis of mania and depression. Lancet 1972, 2, 632-635

Jewett RE und Norton S (1966): Effects of Some Stimulant and Depressant Drugs on Sleep Cycles of Cats. Exp Neurol 15, 463-474

Jung CM, Melanson EL, Frydendall EJ, Perreault L, Eckel RH und Wright KP (2011): Energy expenditure during sleep, sleep deprivation and sleep following sleep deprivation in adult humans. J Physiol $\underline{589}, 235-244$

Kales J, Preston TA und Tan TL (1969): Effects of cigarette withdrawal on sleep and dream patterns. Electroencephalogr Clin Neurophysiol 27, 710

Kales J, Allen C, Preston TA, Tan TL und Kales A (1971): Changes in REM Sleep and Dreaming with Cigarette Smoking and Following Withdrawal. Psychophysiology 7, 347-348

Kershbaum A, Pappajohn DJ, Bellet S, Hirabayashi M und Shafiiha H (1968): Effect of Smoking and Nicotine on Adrenocortical Secretion. JAMA 203, 275-278

Kirschbaum C, Strasburger CJ und Langkrär J (1993): Attenuated Cortisol Response to Psychological Stress but not to CRH or Ergometry in Young Habitual Smokers. Pharmacol Biochem Behav $\underline{44}$, 527-531

Kirschbaum C, Wüst S und Strasburger CJ (1992): "Normal"cigarette smoking increases free cortisol in habitual smokers. Life Sci $\underline{50}, 435-442$

Koella WP: Die Physiologie des Schlafes. Gustav Fischer Verlag, Stuttgart 1988

Kotlyar M, Brauer LH, al'Absi M, Adson DE, Robiner W, Thuras P, Harris J, Finocchi ME, Bronars CA, Candell S et al. (2006): Effect of bupropion on physiological measures of stress in smokers

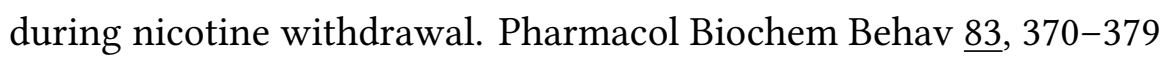

Kotterba S und Schäfer D: Neurologisch-psychiatrische Erkrankungen und Schlaf. In: Rasche K, Sanner B, Schläfer T, Schläfke ME, Sturm A, Zidek W und Schultze-Werninghaus G, Schlafbezo- 
gene Atmungsstörungen in Klinik und Praxis. Blackwell Wissenschafts-Verlag, Berlin 1999, Seite $165-181$

Kröger CB und Lohmann B: Tabakkonsum und Tabakabhängigkeit. Hogrefe Verlag, Göttingen 2007

Kurogochi Y, Nakashima T, Kita T und Nakanishi Y (1985): Influences of free intake of nicotine on several circadian rhythms in rats. Nippon Yakurigaku Zasshi 86, 35-39

Lee AM, Miksys S, Palmour R und Tyndale RF (2006): CYP2B6 is expressed in African Green monkey brain and is induced by chronic nicotine treatment. Neuropharmacology $\underline{50}, 441-450$

Le Houezec J (2003): Role of nicotine pharmacokinetics in nicotine addiction and nicotine replacement therapy: a review. Int J Tuberc Lung Dis $\underline{7}, 811-819$

Léna C, Popa D, Grailhe R, Escourrou P, Changeux JP und Adrien J (2004): Beta2-Containing Nicotinic Receptors Contribute to the Organization of Sleep and Regulate Putative MicroArousals in Mice. J Neurosci $\underline{24}, 5711-5718$

Lesch OM und Walter H: Alkohol und Tabak: medizinische und soziologische Aspekte von Gebrauch, Missbrauch und Abhängigkeit. Springer Verlag, Wien 2009

Levin ED, Conners CK, Silva D, Hinton SC, Meck WH, March J und Rose JE (1998): Transdermal nicotine effects on attention. Psychopharmacology (Berl) 140, 135-141

Loomis S und Gilmour G (2010): Corticosterone urinalysis and nicotinic receptor modulation in rats. J Neurosci Methods $\underline{188}, 243-249$

Lutfy K, Brown MC, Nerio N, Aimiuwu O, Tran B, Anghel A und Friedman TC (2006): Repeated stress alters the ability of nicotine to activate the hypothalamic-pituitary-adrenal axis. J Neurochem 99, 1321-1327

Mackay J und Eriksen M: The Tobacco Atlas. World Health Organization, Genf 2002

Maskos U, Granon S, Faure P und Changeux JP: Nicotinic acetylcholine receptor functions in the CNS investigated with a novel method of stereotaxic gene re-expression in knockout mice. In: Novartis Foundation, Understanding nicotine and tobacco addiction. John Wiley und Sons, Ltd, Chichester 2006, Seite 64-82 
Matta SG, Beyer HS, McAllen KM und Sharp BM (1987): Nicotine Elevates Rat Plasma ACTH by a Central Mechanism. J Pharmacol Exp Ther 243, 217-226

Matta SG, McAllen KM und Sharp BM (1990a): Role of the Fourth Cerebroventricle in Mediating Rat Plasma ACTH Responses to Intravenous Nicotine. J Pharmacol Exp Ther 252, 623-630

Matta SG, Singh J und Sharp BM (1990b): Catecholamines Mediate Nicotine-Induced Adrenocorticotropin Secretion via Alpha-Adrenergic Receptors. Endocrinology 127, 1646-1655

Mayers AG und Baldwin DS (2005): Antidepressants and their effect on sleep. Hum Psychopharmacol 20, 533-559

McCarley RW und Hobson JA (1975): Neuronal Excitability Modulation over the Sleep Cycle: A Structural and Mathematical Model. Science $\underline{189}$, 58-60

McGue M, Elkins I und Iacono WG (2000): Genetic and Environmental Influences on Adolescent Substance Use und Abuse. Am J Med Genet 96, 671-677

Meliska CJ, Stunkard ME, Gilbert DG, Jensen RA und Martinko JM (1995): Immune function in cigarette smokers who quit smoking for 31 days. J Allergy Clin Immunol 95, 901-910

Mellon RD und Bayer BM (1999): The Effects of Morphine, Nicotine and Epibatidine on Lymphocyte Activity and Hypothalamic-Pituitary-Adrenal Axis Responses. J Pharmacol Exp Ther 288, 635642

Mihailescu S, Guzmán-Marín R und Drucker-Colín R (2001): Nicotine stimulation of dorsal raphe neurons: effects on laterodorsal and pedunculopontine neurons. Eur Neuropsychopharmacol $\underline{11}$, $359-366$

Moidel MA, Belz EE, Czambel RK, Rubin RT und Rhodes ME (2006): Novel in vitro perfusion system for the determination of hypothalamic-pituitary-adrenal axis responses. J Pharmacol Toxicol Methods $\underline{53}, 264-271$

Moreno-Coutiño A, Calderón-Ezquerro C und Drucker-Colín R (2007): Long-term changes in sleep and depressive symptoms of smokers in abstinence. Nicotine Tob Res $\underline{9}, 389-396$ 
Morse DE (1989): Neuroendocrine responses to nicotine and stress: enhancement of peripheral stress responses by the administration of nicotine. Psychopharmacology (Berl) $\underline{98}, 539-543$

Newhouse PA, Sunderland T, Narang PK, Mellow AM, Fertig JB, Lawlor BA and Murphy DL (1990): Neuroendocrine, physiologic, and behavioral responses following intravenous nicotine in nonsmoking healthy volunteers and in patients with Alzheimer's disease. Psychoneuroendocrinology $\underline{15}, 471-484$

Niaura R (2008): Nonpharmacologic Therapy for Smoking Cessation: Characteristics and Efficacy of Current Approaches. Am J Med 121, S11-S19

Noble EP, Jeor STS, Ritchie T, Syndulko K, Jeor SCS, Fitch RJ, Brunner RL and Sparkes R (1994): D2 Dopamine Receptor Gene and Cigarette Smoking: A Reward Gene? Med Hypotheses $\underline{42}$, $257-260$

Ohayon MM und Roth T (2002): Prevalence of restless legs syndrome and periodic limb movement disorder in the general population. J Psychosom Res $\underline{53}, 547-554$

Okamoto M, Kita T, Okuda H, Tanaka T und Nakashima T (1992): Effects of Acute Administration of Nicotine on Convulsive Movements and Blood Levels of Corticosterone in Old Rats. Jpn J Pharmacol $60,381-384$

Page F, Coleman G und Conduit R (2006): The effect of transdermal nicotine patches on sleep and dreams. Physiol Behav $\underline{88}, 425-432$

Pangritz D: "Nicotin", aus Thieme RÖMPP Online, September 2003 (www.roempp.com)

Parsons LC und Hamme S (1976): The effects of nicotine withdrawal on the sleep awake cycle. Va J Sci $\underline{27}, 86$

Pauly JR, Grun EU und Collins AC (1992): Tolerance to nicotine following chronic treatment by injections: a potential role for corticosterone. Psychopharmacology (Berl) 108, 33-39

Pentkowski NS, Painter MR, Thiel KJ, Peartree NA, Cheung TH, Deviche P, Adams M, Alba J und Neisewander JL (2011): Nicotine-induced plasma corticosterone is attenuated by social interactions in male and female adolescent rats. Pharmacol Biochem Behav 100, 1-7 
Perkins KA, Lerman C, Coddington S, Jetton C, Karelitz JL, Wilson A, Jennings JR, Ferrell R, Bergen AW und Benowitz NL (2008): Gene and gene by sex associations with initial sensitivity to nicotine in nonsmokers. Behav Pharmacol $\underline{19}, 630-640$

Pickworth WB, Baumann MH, Fant RV, Rothman RB und Henningfield JE (1996): Endocrine Responses During Acute Nicotine Withdrawal. Pharmacol Biochem Behav 55, 433-437

Pollmächer T und Lauer C: Physiologie von Schlaf und Schlafregulation. In: Berger M, Handbuch des normalen und gestörten Schlafs. Springer Verlag, Berlin 1992, Seite 1-44

Pomerleau CS, Garcia AW, Pomerleau OF und Cameron OG (1992): The effects of menstrual phase and nicotine abstinence on nicotine intake and on biochemical and subjective measures in women smokers: a preliminary report. Psychoneuroendocrinology 17, 627-638

Pomerleau OF, Pomerleau CS und Marks JL (2000): Abstinence effects and reactivity to nicotine during 11 days of smoking deprivation. Nicotine Tob Res $\underline{2}, 149-157$

Pomerleau OF, Pomerleau CS, Snedecor SM, Gaulrapp S, Brouwer RN und Cameron OG (2004): Depression, smoking abstinence and HPA function in women smokers. Hum Psychopharmacol $\underline{19}, 467-476$

Pontieri FE, Tanda G, Orzi F und Di Chiara G (1996): Effects of nicotine on the nucleus accumbens and similarity to those of addictive drugs. Nature $\underline{382}, 255-257$

Porcu P, Sogliano C, Cinus M, Purdy RH, Biggio G und Concas A (2003): Nicotine-induced changes in cerebrocortical neuroactive steroids and plasma corticosterone concentrations in the rat. Pharmacol Biochem Behav $\underline{74}, 683-690$

Pötschke-Langer M, Mons U, Schaller K, Stein S, Kahnert S, Schneider NK, Nair U, Schunk S und Mersmann H: Tabakatlas Deutschland 2009. Steinkopff Verlag, Heidelberg 2009

Prosise GL, Bonnet MH, Berry RB und Dickel MJ (1994): Effects of abstinence from smoking on sleep and daytime sleepiness. Chest $\underline{105}, 1136-1141$

Puddey IB, Vandongen R, Beilin LJ und English D (1984): Haemodynamic and neuroendocrine consequences of stopping smoking-a controlled study. Clin Exp Pharmacol Physiol 11, 423-426 
Rasmusson AM, Wu R, Paliwal P, Anderson GM und Krishnan-Sarin S (2006): A decrease in the plasma DHEA to cortisol ratio during smoking abstinence may predict relapse: a preliminary study. Psychopharmacology (Berl) $\underline{186}, 473-480$

Rechtschaffen A und Kales A: A manual of standardized terminology, techniques and scoring system for sleep stages of human subjects. BIS/BRI, UCLA, Los Angeles 1968

Rhodes ME, O’Toole SM, Czambel RK und Rubin RT (2001): Male-female differences in rat hypothalamic-pituitary-adrenal axis responses to nicotine stimulation. Brain Res Bull $\underline{54}$ $681-688$

Rist F und Watzl H: Psychologische Ansätze. In: Gastpar M, Mann K und Rommelspacher H, Lehrbuch der Suchterkrankungen. Georg Thieme Verlag, Stuttgart 1999, Seite 39-49

Rodenbeck A (2011): Biologische Grundlagen des Schlafens und Wachens. Bundesgesundheitsblatt Gesundheitsforschung Gesundheitsschutz $\underline{54}$, 1270-1275

Rodenbeck A, Huether G, Rüther E und Hajak G (2002): Interactions between evening and nocturnal cortisol secretion and sleep parameters in patients with severe chronic primary insomnia. Neurosci Lett $\underline{324}, 159-163$

Rohleder N und Kirschbaum C (2006): The hypothalamic-pituitary-adrenal (HPA) axis in habitual smokers. Int J Psychophysiol 59, 236-243

Saint-Mleux B, Eggermann E, Bisetti A, Bayer L, Machard D, Jones BE, Mühlethaler M und Serafin M (2004): Nicotinic Enhancement of the Noradrenergic Inhibition of Sleep-Promoting Neurons in the Ventrolateral Preoptic Area. J Neurosci 24, 63-67

Salín-Pascual RJ (2002): Relationship between mood improvement and sleep changes with acute nicotine administration in non-smoking major depressed patients. Rev Invest Clin $\underline{54}$, 36-40

Salín-Pascual RJ und Drucker-Colín R (1998): A novel effect of nicotine on mood and sleep in major depression. Neuroreport $\underline{9}, 57-60$

Salín-Pascual RJ und Galicia-Polo L (1999): REM Sleep Latency in Major Depressed Patients Predicts Mood Improvement After Transdermal Nicotine Administration. Sleep Hypn. 1, 32-34 
Salín-Pascual RJ, de la Fuente JR, Galicia-Polo L und Drucker-Colín R (1995): Effects of transderman nicotine on mood and sleep in nonsmoking major depressed patients. Psychopharmacology (Berl) 121, 476-479

Salín-Pascual RJ, Moro-Lopez ML, Gonzalez-Sanchez H und Blanco-Centurion C (1999): Changes in sleep after acute and repeated administration of nicotine in the rat. Psychopharmacology (Berl) $\underline{145}, 133-138$

Saper CB, Chou TC und Scammell TE (2001): The sleep switch: hypothalamic control of sleep and wakefulness. Trends Neurosci $\underline{24}, 726-731$

Saper CB, Scammell TE und Lu J (2005): Hypothalamic regulation of sleep and circadian rhythms. Nature $\underline{437}, 1257-1263$

Schläfke ME und Schäfer T: Physiologie des Schlafes. In: Rasche K, Sanner B, Schläfer T, Schläfke ME, Sturm A, Zidek W und Schultze-Werninghaus G, Schlafbezogene Atmungsstörungen in Klinik und Praxis. Blackwell Wissenschafts-Verlag, Berlin 1999, Seite 17-34

Schläfke ME und Schäfer T: Physiologie des Schlafes. In: Rasche K und Schultze-Werninghaus, Update Schlafmedizin, 2. Aufl. Dustri-Verlag Feistle, München 2009, Seite 1-12

Schunack W (2008): Nichtrauchen, ein Essential für eine gesunde Lunge. Pharm. Unserer Zeit 37, 494-499

Seifert J, Seifert E, Brechtelsbauer H, Kurz C und Thurau K (1984): The influence of chronic subcutaneous nicotine administration on aldosterone and corticosterone plasma concentrations and the plasma renin activity. Klin Wochenschr $\underline{62}, 81-85$

Sellini M, Sartori MP, Baccarini S, Bassi R und Dimitriadis E (1989a): ACTH and cortisol after cigarette smoke exposure during the dexamethasone suppression test in smokers and nonsmokers. Boll Soc Ital Biol Sper $\underline{65}, 377-380$

Sellini M, Sartori MP, Letizia C, Dimitriadis E, Bassi R und Baccarini S (1989b): Changes in the levels of ACTH and cortisol after passive exposure to cigarette smoke in smokers and non-smokers. Boll Soc Ital Biol Sper $\underline{65}, 365-369$ 
Sellini M, Sartori MP, Letizia C, Dimitriadis E, Bassi R und Baccarini S (1989c): Oral administration of nicotine and plasma levels of ACTH and cortisol in smokers and non-smokers. Boll Soc Ital Biol Sper $\underline{65}, 371-375$

Semba J, Wakuta M, Maeda J und Suhara T (2004): Nicotine withdrawal induces subsensitivity of hypothalamic-pituitary-adrenal axis to stress in rats: implications for precipitation of depression during smoking cessation. Psychoneuroendocrinology 29, 215-226

Seyler LE, Fertig J, Pomerleau O, Hunt D und Parker K (1984): The effects of smoking on ACTH and cortisol secretion. Life Sci $\underline{34}, 57-65$

Sharp BM und Beyer HS (1986): Rapid Desensitization of the Acute Stimulatory Effects of Nicotine on Rat Plasma Adrenocorticotropin and Prolactin. J Pharmacol Exp Ther 238, 486-491

Shiromani PJ und Gillin C (1987): Acetylcholin and the regulation of REM sleep: basic mechanisms and clinical implications for affective illness and narcolepsy. Ann. Rev. Pharmacol. Toxicol. 27, $137-156$

Skwara AJ, Karwoski TE, Czambel RK, Rubin RT und Rhodes ME (2012): Influence of environmental enrichment on hypothalamic-pituitary-adrenal (HPA) responses to single-dose nicotine, continuous nicotine by osmotic mini-pumps, and nicotine withdrawal by mecamylamine in male and female rats. Behav Brain Res 234, 1-10

Smolka MN, Budde H, Karow AC und Schmidt LG (2004): Neuroendocrinological and neuropsychological correlates of dopaminergic function in nicotine dependence. Psychopharmacology (Berl) $175,374-381$

Soldatos CR, Kales JD, Scharf MB, Bixler EO und Kales A (1980): Cigarette Smoking Associated with Sleep Difficulty. Science 207, 551-553

Soloway SB (1976): Naturally Occurring Insecticides. Environ Health Perspect $\underline{14}, 109-117$

Song C, Lin A, Bonaccorso S, Heide C, Verkerk R, Kenis G, Bosmans E, Scharpe S, Whelan A und Cosyns P (1998): The inflammatory response system and the availability of plasma tryptophan in patients with primary sleep disorders and major depression. J Affect Disord $\underline{49}, 211-219$ 
Staner L, Luthringer R, Dupont C, Aubin HJ und Lagrue G (2006): Sleep effects of a 24-h versus a 16-h nicotine patch: a polysomnographic study during smoking cessation. Sleep Med $\underline{7}, 147-154$

Statistisches Bundesamt: Nichtraucher auf dem Vormarsch - Gesundheitsschutz hat Vorrang. Statistisches Bundesamt, Wiesbaden 2010

Statistisches Bundesamt: Mikrozensus - Fragen zur Gesundheit - Rauchgewohnheiten der Bevölkerung 2009. Statistisches Bundesamt, Wiesbaden 2011

Suzuki T, Ikeda H, Narita S, Shibata O, Waki S und Egashira K (1973): Adrenal cortical secretion in response to nicotine in conscious and anaesthetized dogs. Q J Exp Physiol Cogn Med Sci $\underline{58}$, $139-142$

Talwar A, Jain M und Vijayan VK (2004): Pharmacotherapy of tobacco dependence. Med Clin North Am 요, 1517-1534

Teneggi V, Tiffany ST, Squassante L, Milleri S, Ziviani L und Bye A (2002): Smokers deprived of cigarettes for $72 \mathrm{~h}$ : effect of nicotine patches on craving and withdrawal. Psychopharmacology (Berl) $\underline{164}, 177-187$

Trenkwalder C: Leitlinie zur Diagnose und Therapie des Restless-Legs-Syndroms (RLS). Georg Thieme Verlag, Stuttgart 2007

Turner DM (1975): The role of adrenal catecholamines in the release of corticosterone and fatty acids by nicotine in the rat. Res Commun Chem Pathol Pharmacol $\underline{12}, 645-655$

U. S. Department of Health and Human Services: The Health Consequences of Smoking: A Report of the Surgeon General. Atlanta 2004

Ussher M, West R, Evans P, Steptoe A, McEwen A, Clow A und Hucklebridge F (2006): Reduction in Cortisol After Smoking Cessation Among Users of Nicotine Patches. Psychosom Med $\underline{68}$, 299-306

Ussher M, Aveyard P, Reid F, West R, Evans P, Clow A, Hucklebridge F, Fuller J, Ibison J und Steptoe A (2011): A randomised placebo-controlled trial of oral hydrocortisone for treating tobacco withdrawal symptoms. Psychopharmacology (Berl) 216, 43-51 
Vazquez J, Guzmán-Marín R, Salín-Pascual RJ and Drucker-Colín R (1996): Transdermal nicotine on sleep and PGO spikes. Brain Res $\underline{737}, 317-320$

Vázquez-Palacios G, Hernández-González M, Guevara Pérez MA und Bonilla-Jaime H (2010): Nicotine and fluoxetine induce arousing effects on sleep-wake cycle in antidepressive doses: A possible mechanism of antidepressant-like effects of nicotine. Pharmacol Biochem Behav $\underline{94}$, 503-509

Velazquez-Moctezuma J, Shalauta MD, Gillin JC und Shiromani PJ (1990): Microinjections of nicotine in the medial pontine reticular formation elicits REM sleep. Neurosci Lett $\underline{115}, 265-268$

Volk S: Schlafstörungen. Springer-Verlag, Berlin 1995

Volkow ND, Wang GJ, Fowler JS, Logan J, Hitzemann R, Ding YS, Pappas N, Shea C und Piscani K (1996): Decreases in dopamine receptors but not in dopamine transporters in alcoholics. Alcohol Clin Exp Res 20, 1594-1598

Volkow ND, Wang GJ, Fowler JS, Logan J, Gatley SJ, Hitzemann R, Chen AD, Dewey SL und Pappas N (1997): Decreased striatal dopaminergic responsiveness in detoxified cocaine-dependent subjects. Nature $\underline{386}, 830-833$

Wang GJ, Volkow ND, Fowler JS, Logan J, Abumrad NN, Hitzemann RJ, Pappas NS and Pascani K (1997): Dopamine D2 Receptor Availability in Opiate-Dependent Subjects before and after Naloxone-Precipitated Withdrawal. Neuropsychopharmacology 16, 174-182

Ward KD, Garvey AJ, Bliss RE, Sparrow D, Young JB und Landsberg L (1991): Changes in Urinary Catecholamine Excretion After Smoking Cessation. Pharmacol Biochem Behav $\underline{40}$, 937-940

Weidenfeld J, Bodoff M, Saphier D und Brenner T (1989): Further studies on the stimulatory action of nicotine on adrenocortical function in the rat. Neuroendocrinology $\underline{50}, 132-138$

Wetter DW und Young TB (1994): The Relation between Cigarette Smoking and Sleep Disturbance. Prev Med 23, 328-334

Wetter DW, Fiore MC, Baker TB und Young TB (1995): Tobacco Withdrawal and Nicotine Replacement Influence Objective Measures of Sleep. J Consult Clin Psychol 63, 658-667 
Wetter DW, Fiore MC, Young TB, McClure JB, de Moor CA und Baker TB (1999): Gender Differences in Response to Nicotine Replacement Therapy: Objective and Subjective Indexes of Tobacco Withdrawal. Exp Clin Psychopharmacol ㅁ, 135-144

Wetter DW, Carmack CL, Anderson CB, Moore CA, De Moor CA, Cinciripini PM and Hirshkowitz M (2000): Tobacco Withdrawal Signs and Symptoms Among Women With and Without a History of Depression. Exp Clin Psychopharmacol $\underline{8}, 88-96$

Wetter TC und Pollmächer T (1997): Restless legs and periodic leg movements in sleep syndromes. Journal of neurology 244, S37-S45

Wilkins JN, Carlson HE, Van Vunakis H, Hill MA, Gritz E und Jarvik ME (1982): Nicotine from Cigarette Smoking Increases Circulating Levels of Cortisol, Growth Hormone, and Prolactin in Male Chronic Smokers. Psychopharmacology (Berl) $\underline{78}$, 305-308

Wolter TD, Hauri PJ, Schroeder DR, Wisbey JA, Croghan IT, Offord KP, Dale LC und Hurt RD (1996): Effects of 24-hr Nicotine Replacement on Sleep and Daytime Activity during Smoking Cessation. Prev Med 25, 601-610

World Health Organization: World Health Statistics 2011. World Health Organization, Genf 2011

Yasuno F, Ota M, Ando K, Ando T, Maeda J, Ichimiya T, Takano A, Doronbekov TK, Fujimura Y, Nozaki S et al. (2007): Role of Ventral Striatal Dopamine D1 Receptor in Cigarette Craving. Biol Psychiatry $\underline{61}, 1252-1259$

Yoshimasu K und Kiyohara C (2003): Genetic Influences on Smoking Behavior and Nicotine Dependence: a Review. J Epidemiol 13, 183-192

Zevin S, Gourlay SG und Benowitz NL (1998): Clinical pharmacology of nicotine. Clin Dermatol $\underline{16}, 557-564$

Zhang L, Samet J, Caffo B und Punjabi NM (2006): Cigarette Smoking and Nocturnal Sleep Architecture. Am J Epidemiol 164, 529-537

Zhang L, Samet J, Caffo B, Bankman I und Punjabi NM (2008): Power Spectral Analysis of EEG Activity During Sleep in Cigarette Smokers. Chest $\underline{133}$, 427-432 
Zhang L, Dong Y, Doyon WM und Dani JA (2012): Withdrawal from Chronic Nicotine Exposure Alters Dopamine Signaling Dynamics in the Nucleus Accumbens. Biol Psychiatry $\underline{71}, 184-191$ 


\section{Abbildungsverzeichnis}

1.1 Schlafprofil einer gesunden Versuchsperson. . . . . . . . . . . . . . . . . . 15

1.2 Das Zwei-Prozess-Modell. . . . . . . . . . . . . . . . . . . . . . 17

1.3 Reziprokes Interaktionsmodell. . . . . . . . . . . . . . . . . . . . . . . . 19

1.4 Polysomnografische Stadien und zugehörige Transmitter. . . . . . . . . . . . . 20 


\section{Tabellenverzeichnis}

2.1 Definitionen der polysomnografischen Stadien. . . . . . . . . . . . . . . . 31

3.1 Anzahl an Suchergebnissen und aufgenommenen Studien. . . . . . . . . . . . . 41

3.2 Anzahl an Studien und Forschungsgruppen. . . . . . . . . . . . . . . . . . . . 42

3.3 Studiendesigns der Studien. . . . . . . . . . . . . . . . . . . . . . . 42

3.4 Auswirkungen einer akuten Nikotingabe auf polysomnografische Parameter bei Katzen. . . . . . . . . . . . . . . . . . . . . 44

3.5 Auswirkungen einer akuten Nikotingabe auf polysomnografische Parameter bei Nagern. . . . . . . . . . . . . . . . . . . . . . . 45

3.6 Auswirkungen einer chronischen Nikotingabe auf polysomnografische Parameter bei Ratten. . . . . . . . . . . . . . . . . . . . . . . . . . . . . . . . 46

3.7 Auswirkungen eines Nikotinentzugs auf polysomnografische Parameter bei Katzen und Ratten. . . . . . . . . . . . . . . . . . . . . . . . . . . . 47

3.8 Auswirkungen einer Nikotingabe auf polysomnografische Parameter in tierexperimentellen Untersuchungen (Zusammenfassung). . . . . . . . . . . . . . . . .

3.9 Auswirkung einer akuten Nikotingabe auf polysomnografische Parameter bei gesunden Nichtrauchern. . . . . . . . . . . . . . . . . . . . . . . .

3.10 Auswirkungen eines Nikotinentzugs auf polysomnografische Parameter bei gesunden Nichtrauchern. . . . . . . . . . . . . . . . . . . . .

3.11 Auswirkung einer akuten Nikotingabe auf polysomnografische Parameter bei depressiven Nichtrauchern. . . . . . . . . . . . . . . . . . . . . .

3.12 Auswirkung einer chronischen Nikotingabe auf polysomnografische Parameter bei depressiven Nichtrauchern. . . . . . . . . . . . . . . . . . . . .

3.13 Auswirkung eines Nikotinentzugs auf polysomnografische Parameter bei depressiven Nichtrauchern. . . . . . . . . . . . . . . . . . . . . . .

3.14 Auswirkungen einer Nikotingabe auf polysomnografische Parameter gesunder Nichtraucher (Zusammenfassung). . . . . . . . . . . . . . . . . .

3.15 Auswirkungen einer Nikotingabe auf polysomnografische Paramter depressiver Nichtraucher (Zusammenfassung). . . . . . . . . . . . . . . . . 55

3.16 Auswirkungen einer Nikotingabe auf die Aktivität im Schlaf bei Nichtrauchern. . 55

3.17 Auswirkung eines mehrtägigen Nikotinentzugs auf polysomnografische Parameter bei Rauchern 1. . . . . . . . . . . . . . . . . . . . . . . . . .

3.18 Auswirkung eines mehrtägigen Nikotinentzugs auf polysomnografische Parameter bei Rauchern 2. 
3.19 Auswirkung eines mehrtägigen Nikotinentzugs auf polysomnografische Parameter bei Rauchern 3.

3.20 Auswirkung eines Langzeit-Nikotinentzugs auf polysomnografische Parameter bei Rauchern.

3.21 Auswirkungen eines Nikotinentzugs auf polysomnografische Parameter bei Rauchern (Zusammenfassung). . . . . . . . . . . . . . . . . . . . . . .

3.22 Auswirkung eines Nikotinentzugs auf die Aktivität im Schlaf bei Rauchern. . . . .

3.23 Auswirkung eines Nikotinentzugs auf das Bindungspotential an Dopaminrezeptoren bei Rauchern. . . . . . . . . . . . . . . . . . . . . . . . .

3.24 Auswirkung eines Nikotinentzugs auf die Dopaminkonzentration im Urin bei Rauchern.

3.25 Auswirkung eines Nikotinentzugs auf den ACT bei Rauchern. . . . . . . . . . . .

3.26 Auswirkung einer akuten Nikotingabe auf die HHN-Achse in tierexperimentellen Untersuchungen $1 \ldots \ldots \ldots \ldots \ldots \ldots$

3.27 Auswirkung einer akuten Nikotingabe auf die HHN-Achse in tierexperimentellen Untersuchungen 2. . . . . . . . . . . . . . . . . . .

3.28 Auswirkung einer akuten Nikotingabe auf die HHN-Achse in tierexperimentellen Untersuchungen 3. . . . . . . . . . . . . . . . . . 66

3.29 Auswirkung einer akuten Nikotingabe auf die HHN-Achse in tierexperimentellen Untersuchungen $4 \ldots \ldots \ldots \ldots \ldots$

3.30 Auswirkung einer akuten Nikotingabe auf die HHN-Achse in tierexperimentellen Untersuchungen $5 \ldots \ldots \ldots \ldots \ldots \ldots$

3.31 Auswirkung einer chronischen Nikotingabe auf die HHN-Achse in tierexperimentellen Untersuchungen 1. . . . . . . . . . . . . . . . . . . . . .

3.32 Auswirkung einer chronischen Nikotingabe auf die HHN-Achse in tierexperimen-

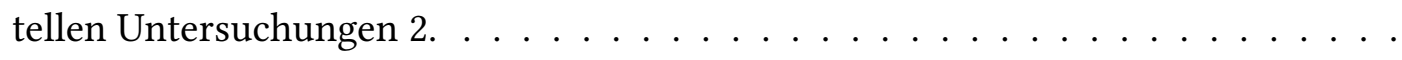

3.33 Auswirkung eines Nikotinentzugs auf die HHN-Achse in tierexperimentellen Un-

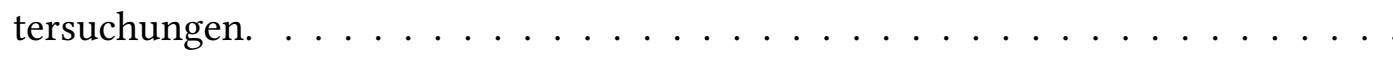

3.34 Auswirkungen einer Nikotingabe auf die HHN-Achse in tierexperimentellen Untersuchungen (Zusammenfassung). . . . . . . . . . . . . . . 72

3.35 Auswirkungen einer Nikotingabe auf die HHN-Achse bei Nichtrauchern. . . . . . 74

3.36 Auswirkungen einer Nikotingabe auf die HHN-Achse bei Nichtrauchern (Zusammenfassung). . . . . . . . . . . . . . . . . 74

3.37 Auswirkung eines mehrstündigen Nikotinentzugs auf die HHN-Achse bei Rauchern. 77

3.38 Auswirkung eines mehrtägigen Nikotinentzugs auf die HHN-Achse bei Rauchern. 79

3.39 Auswirkung eines mehrwöchigen Nikotinentzugs auf die HHN-Achse bei Rauchern. 80

3.40 Auswirkungen eines Nikotinentzugs auf die HHN-Achse bei Rauchern (Zusam-

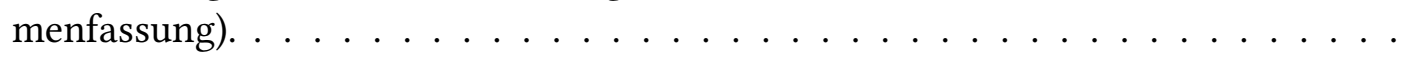

6.1 Nikotingabe und objektive Schlafparameter in tierexperimentellen Untersuchungen (Übersicht). . . . . . . . . . . . . . . . . . . . . . . . 128

6.2 Nikotingabe und objektive Schlafparameter bei Nichtrauchern (Übersicht). . . . . 129

6.3 Nikotinentzug und objektive Schlafparameter bei Rauchern (Übersicht). . . . . . . 130

6.4 Nikotinentzug und dopaminerge Parameter bei Rauchern (Übersicht). . . . . . . . 132

6.5 Nikotingabe und HHN-Achse in tierexperimentellen Untersuchungen (Übersicht). 133 
6.6 Nikotingabe und HHN-Achse bei Nichtrauchern (Übersicht). . . . . . . . . . . . . 137

6.7 Nikotinentzug und HHN-Achse bei Rauchern (Übersicht). . . . . . . . . . . . . 138 


\section{Danksagung}

Meiner Doktormutter Frau Prof. Dr. Andrea Rodenbeck möchte ich für die Bereitstellung des Dissertationsthemas und die hervorragende Betreuung herzlich danken. Ihre mühevolle Unterstützung und Förderung hat mir nicht nur bei der Verfassung der Dissertation sehr geholfen, sondern auch in beruflichen Belangen. Bedanken möchte ich mich insbesondere für ihre ständige freundliche Bereitschaft, mir zu helfen und mich zu ermutigen.

Meinen Eltern danke ich sehr für ihre permanente Hilfe und Unterstützung. Auch für das Korrekturlesen möchte ich mich bei ihnen und meinem Bruder herzlich bedanken.

Adam danke ich für seine großartige und geduldige Hilfe bei der Arbeit mit ETEX, für seinen fachlichen Rat und dafür, dass er immer für mich da ist. 